\title{
Aerofoil geometry effects on turbulence interaction noise
}

\section{Chaitanya Paruchuri}

A thesis submitted in fulfillment of the requirements

for the degree of Doctor of Philosophy

in the

Faculty of Engineering and the Environment

Institute of Sound and Vibration Research

April 2017 



\title{
UNIVERSITY OF SOUTHAMPTON
}

\author{
Abstract \\ Faculty of Engineering and the Environment \\ Institute of Sound and Vibration Research \\ Doctor of Philosophy
}

Aerofoil geometry effects on turbulence interaction noise

by Chaitanya PARUCHURI

Fan broadband is one of the dominant noise sources on an aircraft engine, particularly at approach. The dominant noise generation mechanism is due to turbulent- aerofoil interaction noise (TAI). This thesis investigates the effect of changes in 2D aerofoil geometry on TAI noise. The main focus of this thesis is to attempt to reduce it through the development of innovative leading edge geometries. The first two chapters of the thesis deals with an experimental and numerical investigation into the effect of aerofoil geometry on interaction noise on single aerofoils and on cascades. Consistent with previous work, they show that variations in aerofoil parameters, such as aerofoil thickness, leading edge nose radius and camber, produce only a small changes in broadband interaction noise at approach conditions. Subsequent chapters deal with the development of innovative leading edge serration profiles aimed at reducing interaction noise. Chapter 4 is a detailed study into the limitations of single-wavelength serrations in reducing interaction noise. The optimum profile is identified. Chapters 5, 6 and 7 all deal with the development of innovative profiles that can provide up to $10 \mathrm{~dB}$ of additional noise reductions compared to single-wavelength serrations. For each of the profiles investigated a simple model is developed to aid the understanding of their interaction mechanism. 



\section{Contents}

$\begin{array}{lll}\text { Abstract } & \text { iii }\end{array}$

Declaration of Authorship $\quad$ xi

Acknowledgements $\quad$ xiii

$\begin{array}{ll}\text { List of Abbreviations } & \text { xv }\end{array}$

List of Symbols $\quad$ xvii

1 Introduction 1

1.1 Motivation ............................ 1

1.2 Turbulence-aerofoil interaction noise . . . . . . . . . . . . . . . . 3

1.2.1 Modeling of OGV response . . . . . . . . . . . . . 3

Analytical \& Experimental studies . . . . . . . . . . . . . . 3

Numerical studies of aerofoil interaction noise . . . . . . . . . 5

1.2.2 Reduction of OGV response . . . . . . . . . . . . . . 6

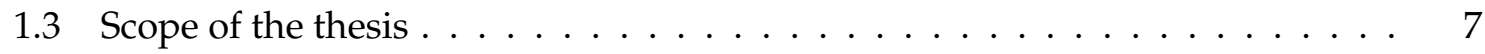

1.4 Original Contribution and Thesis structure . . . . . . . . . 7

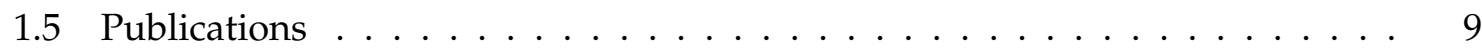

1.5.1 Journal Papers . . . . . . . . . . . . . . . . . . . 9

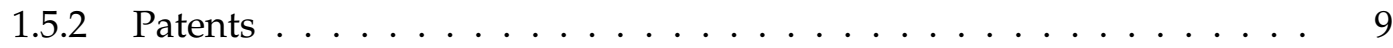

1.5.3 Conference Papers till April 2017 . . . . . . . . . . . . . . . . 10

2 Turbulence-aerofoil interaction (TAI) noise 11

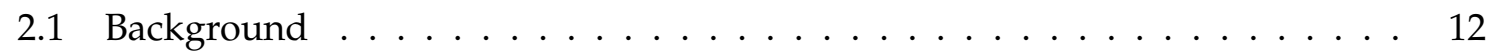

2.2 Flat plate validation . . . . . . . . . . . . . . . . . . 16

2.3 Aerofoil Geometry effects . . . . . . . . . . . . . . . . . . . . . . 20

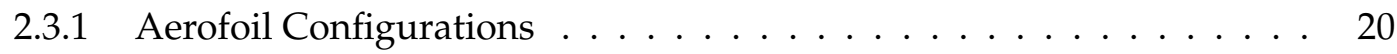

2.3 .2 Influence of Self-Noise . . . . . . . . . . . . . . . . . . 21

2.3.3 Effect on interaction noise due to thickness . . . . . . . . . . . 22 
2.3.4 Comparison of thickness-based empirical models for the prediction on interaction from aerofoils . . . . . . . . . . . . . 24

2.3.5 Effect on interaction noise due to leading edge nose radius . . . . . . 27

2.3.6 Discussion on thickness and nose radius effects . . . . . . . . . . . 29

2.3.7 Effect of camber on turbulence-aerofoil interaction noise . . . . . . . 29

2.3.8 Effect of AOA on turbulence-aerofoil interaction noise . . . . . . . 30

2.4 Effects on interaction noise due to flow speed $\ldots \ldots \ldots \ldots$

2.5 Comparisons with CAA Simulations . . . . . . . . . . . . . . . 33

2.6 Flow measurement . . . . . . . . . . . . . . . . . . . . . 35

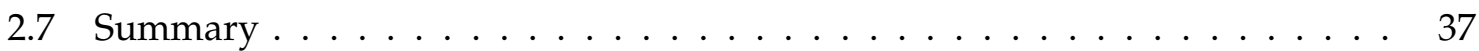

3 Turbulence-Airfoil interaction noise in cascades 39

3.1 Background . . . . . . . . . . . . . . . . . . . 40

3.2 Scope of the chapter . . . . . . . . . . . . . . . . . . . . . . . . 40

3.3 Fourier description of the radiated sound power from 2D aerofoil cascades . $\quad 42$

3.4 Numerical Procedure . . . . . . . . . . . . . . . . . . . . . . . . . . . . 45

3.4 .1 Steady flow simulations . . . . . . . . . . . . . . 46

3.4 .2 Linear unsteady CFD simulations . . . . . . . . . . . . . 46

3.4.3 Post-processing for upstream and downstream noise . . . . . . . . . 46

3.5 Validation on Flat Plate annular cascade . . . . . . . . . . . . . . . . . 46

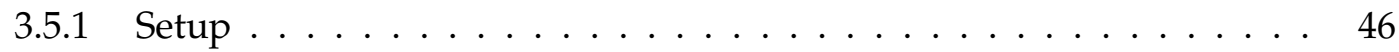

3.5 .2 Results . . . . . . . . . . . . . . . . . . 47

Single incoming mode validation . . . . . . . . . . . . . . . 47

Multiple incoming modes at fixed frequency . . . . . . . . . . . . 48

Validation of periodicity . . . . . . . . . . . . . . . . . 49

Broadband Validation . . . . . . . . . . . . . . . . . . . . . 49

3.6 Realistic aerofoils . . . . . . . . . . . . . . . . . . . 51

3.6 .1 Mesh topology . . . . . . . . . . . . . . 51

3.6 .2 Typical Mean Flow . . . . . . . . . . . . . . . . 52

3.6.3 Zero-cambered aerofoil . . . . . . . . . . . . . 52

Comparison with Flat plate LINSUB solution . . . . . . . . . . . . 53

Influence of viscous mean flow on aerofoil noise generation $\ldots . . .53$

Modal convergence . . . . . . . . . . . . . . . . . . . 55

Influence of aerofoil thickness on interaction noise . . . . . . . . . . 57

Effect of aerofoil nose radius _ . . . . . . . . . . . . . . 60 
3.6.4 Cambered aerofoils . . . . . . . . . . . . . . . . . . 62

Influence of aerofoil camber on interaction noise . . . . . . . . . 62

Influence of aerofoil thickness on cambered aerofoil . . . . . . . . . 64

Broadband comparison for cambered aerofoil . . . . . . . . . 66

Influence of Flow incidence on interaction noise . . . . . . . . . . 66

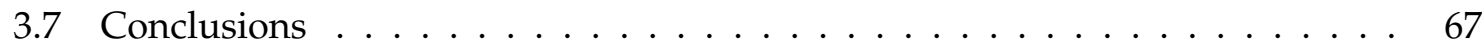

4 Turbulence-aerofoil interaction noise reduction using sinusoidal serrations $\quad 69$

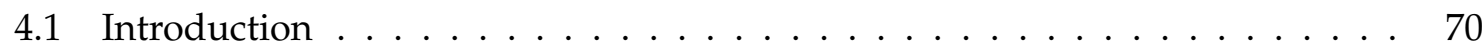

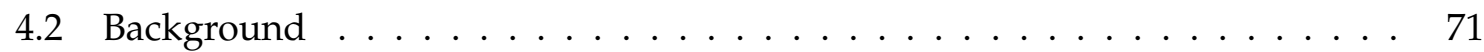

4.3 Aerofoil models . . . . . . . . . . . . . . . . . . . . . . . 74

4.3.1 Flat plate aerofoils . . . . . . . . . . . . . . . . 74

4.3 .2 3D Aerofoil models . . . . . . . . . . . . . . . . . . . . . . . 75

4.4 Acoustic Performance . . . . . . . . . . . . . . . . . . . . 76

4.4.1 Overall acoustic performance on flat-plates . . . . . . . . . . 76

4.4.2 Sound power reductions versus non-dimensional frequency $f h / U$. . 76

4.4 .3 Optimal serration wavelength $\ldots \ldots \ldots$. . . . . . . . 77

4.4 .4 Interpretation $\ldots \ldots \ldots \ldots \ldots \ldots$

Derivation of optimum wavelength $\lambda_{0}=4 \Lambda_{t} \ldots \ldots \ldots$. . . . 80

Geometric similarity interpretation of Strouhal number scaling . . . . 82

Phase model for the prediction noise reduction spectra at the optimum wavelength .................. 83

Balance between source strength per valley and number of valleys . . 85

4.4 .5 Noise reduction contours . . . . . . . . . . . . . . . 86

4.4.6 Influence of self-noise on aerofoils on 3D aerofoils . . . . . . . . . . 88

4.4.7 Influence of leading edge serrations on trailing edge self-noise . . . . 88

4.4.8 Validity of optimal serration angles for 3D aerofoils . . . . . . . . . 90

4.4.9 Comparison with serrated flat plate . . . . . . . . . . . . . . 91

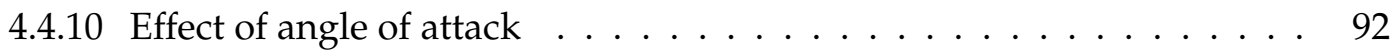

4.5 Aerodynamic Measurement . . . . . . . . . . . . . . . . . . 93

4.6 Flow measurement . . . . . . . . . . . . . . . . . . . . . . . 94

4.6 .1 Mean flow . . . . . . . . . . . . . . . . . . . . 94

4.6.2 Effect on leading edge serrations on boundary layer development . . . 95

4.6 .3 Wake characteristics . . . . . . . . . . . . . . . . . . . 97

4.7 Summary . . . . . . . . . . . . . . . . . . . . . 98 
5 Noise reductions through double-wavelength leading edge serrations; a new control concept

5.1 Introduction . . . . . . . . . . . . . . . . . . . . 102

5.2 Double-wavelength leading edge serrations . . . . . . . . . . . . . . . . 103

5.3 Flat plates leading edge serrations . . . . . . . . . . . . . . . . . . . 104

5.4 Comparison of noise reductions due to double-wavelength and single-wavelength serrations . . . . . . . . . . . . . . . . . . . . . . 105

5.5 Sensitivity on noise reductions due to $h_{r}$ and $\lambda_{r} \ldots \ldots \ldots \ldots$ 5.5.1 Influence on noise reductions due to $h_{r} \ldots \ldots \ldots \ldots$

5.5.2 Influence on noise reductions due to $\lambda_{r} \ldots \ldots \ldots$. . . . . . . 107

5.6 Simple analytical model for additional noise reductions . . . . . . . . . . . . 110

5.7 Self-similarity of double-wavelength serrations . . . . . . . . . . . . . . . . 113

5.8 Predicted dependence of $\Delta \mathrm{PWL}_{I}$ on $h_{r}$ and $\lambda_{r}$ on noise reductions $\ldots \ldots$

5.8.1 Noise reductions at the tuned frequency $\omega_{0} \ldots \ldots$. . . . . . . 115

5.8 .2 Optimum condition $\left(\omega_{0}=\omega_{\Lambda}\right) \ldots \ldots \ldots \ldots \ldots$

5.8 .3 Noise reduction frequency bandwidth . . . . . . . . . . . . . . 116

5.9 Sound Power Spectra comparison for 3-D aerofoils . . . . . . . . . . . . . . . 118

5.10 Aerodynamic performance . . . . . . . . . . . . . . . . . . . . . . . . . . 119

5.11 Conclusion . . . . . . . . . . . . . . . . . . . . 120

6 Slitted leading edge profiles for the reduction of turbulence-aerofoil interaction noise 123

6.1 Background . . . . . . . . . . . . . . . . . . . . . 124

6.2 Acoustic performance of sawtooth serrations . . . . . . . . . . . . . . . . . 124

6.3 Slitted-root profiles . . . . . . . . . . . . . . . . . . . . 125

6.3 .1 Geometry . . . . . . . . . . . . . . . . . . . . 125

6.3.2 A simple analytical model to predict noise reductions . . . . . . . . . 127

6.3.3 Limiting case . . . . . . . . . . . . . . . . . . . . . . . . . 129

Slit width tends to zero . . . . . . . . . . . . . . . . . . . 130

Optimum slit geometry . . . . . . . . . . . . . . . . 130

6.3.4 Acoustic performance on flat plate . . . . . . . . . . . . . . 130

6.3.5 Influence of $w$ on additional noise reductions . . . . . . . . . . . 131

6.3.6 Influence of $h_{s}$ on additional noise reductions $\ldots \ldots \ldots$

6.3.7 Comparison between predicted and measured additional noise reductions . . . . . . . . . . . . . . . . . . . . 135

6.3.8 Sound Power Spectra comparison for 3D aerofoils . . . . . . . . . . . . 137 
6.4 Slitted profiles . . . . . . . . . . . . . . . . . 138

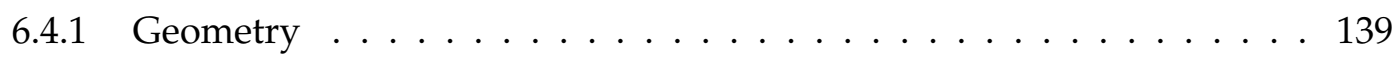

6.4.2 Influence of $a$ and $w$ on noise reductions f . . . . . . . . . 140

6.4 .3 Optimum sound power redictions . . . . . . . . . . . . . . . 141

6.4.4 Sound Power Spectra comparison for 3D aerofoils . . . . . . . . . . . 141

6.5 V-slit serrations . . . . . . . . . . . . . . . . . . . . . . . . . . 142

6.5 .1 Geometry . . . . . . . . . . . . . . . . . . 143

6.5.2 Acoustic performance on flat plate aerofoils . . . . . . . . . . . . . 143

6.6 Conclusions . . . . . . . . . . . . . . . . . . . . . . . . . . 144

7 Enhanced noise reductions using variable-slit and chopped-peak leading edge ser$\begin{array}{ll}\text { rations } & 147\end{array}$

7.1 Background . . . . . . . . . . . . . . . . . . . . 147

7.2 Variable-slits serrations . . . . . . . . . . . . . . . . . . . . . . . . . . 149

7.2.1 Geometry . . . . . . . . . . . . . . . . . . . . . . 149

7.2.2 Control principle . . . . . . . . . . . . . . . . . . . . . . . . . . . 149

7.2.3 Acoustic performance on flat plates . . . . . . . . . . . . 150

7.2.4 Influence on noise reductions due to $a$ and $b \ldots \ldots$. . . . . 150

7.2.5 Simplified analytical model to predict additional noise reductions . . . 152

7.2.6 Influence on noise reductions due to flow speed . . . . . . . . . . . 155

7.2.7 Predicted dependence of $\Delta \mathrm{PWL}_{I}$ on $N$ and $\lambda$ on noise reductions . . 155

7.3 Chopped-peak serrations . . . . . . . . . . . . . . . . . 156

7.3 .1 Geometry . . . . . . . . . . . . . . . . . . 157

7.3.2 Control principle . . . . . . . . . . . . . . . . . . . . 157

7.3.3 Acoustic performance on flat plate aerofoils . . . . . . . . . . . . 158

7.3.4 Influence of noise reductions due to flow speed . . . . . . . . . 158

7.3.5 Simple analytical model to predict additional noise reductions . . . . . 159

7.4 Independence of low frequency noise reductions to serration geometry . . . . 160

8 Conclusions and Future work $\quad 163$

8.1 Conclusions . . . . . . . . . . . . . . . . . . . . . . 163

8.1.1 General Conclusions . . . . . . . . . . . . . . . . . . . . 163

8.1 .2 Specific Conclusions . . . . . . . . . . . . . . . . . 164

8.1.3 Comparison of different serration profiles . . . . . . . . . . . 165

8.2 Future work . . . . . . . . . . . . . . . . . . . . . . 166 
$\begin{array}{ll}\text { A Experimental setup } & 169\end{array}$

A.1 Open-Jet wind tunnel . . . . . . . . . . . . . . . . . . . . . . . . . . . 169

A.2 Measurement techniques . . . . . . . . . . . . . . . . . . . . . . 170

A.2.1 Far-field noise measurement . . . . . . . . . . . . . . . 170

A.2.2 Characterization of the jet with turbulence grid . . . . . . . . . 170

A.2.3 Hot-wire measurement . . . . . . . . . . . . . . . . 171

A.2.4 Particle Image Velocimetry (PIV) setup $\ldots \ldots \ldots$. . . . . . . . . 173

A.2.5 Aerodynamic measurements . . . . . . . . . . . . . . . . . 173

A.3 Measuring TE noise and LE noise . . . . . . . . . . . . . . . . . . . . 174

$\begin{array}{ll}\text { B Analytical Flat plate solution } & 177\end{array}$

B.1 Amiet's flat plate turbulence-aerofoil interaction model . . . . . . . . . . . . 177

B.1.1 Model formulation . . . . . . . . . . . . . . . . . . . . . 177

B.2 Importance of subcritical and supercritical gust frequencies . . . . . . . . . . 179

C HYDRA Setup 183

C.1 Process Map . . . . . . . . . . . . . . . . . . . . . . . 183

C.1.1 Meshing using PADRAM . . . . . . . . . . . . . . . 183

C.1.2 JM51 . . . . . . . . . . . . . . . . . . . . 183

C.1.3 JM52 . . . . . . . . . . . . . . . . . . . . . . 184

C.1.4 JM56 . . . . . . . . . . . . . . . . . . . . . . . . . 184

C.2 Stability of Linear Unsteady solver . . . . . . . . . . . . . . . . 185

C.2.1 The Generalized Minimal Residual algorithm _. . . . . . . . . . . 185

C.2.2 The Recursive Projection Method . . . . . . . . . . . . . . . . . 185

C.3 Grid definition and setup $\ldots \ldots \ldots \ldots \ldots \ldots \ldots$

$\begin{array}{ll}\text { D Additional serration results } & 189\end{array}$

D.1 Modifying realistic OGV geometry for BB reduction . . . . . . . . . . . . 189

D.2 Leading edge serration geometries . . . . . . . . . . . . . . . 190

D.2.1 Random serrations . . . . . . . . . . . . . . . . . . . . . . . 190

D.2.2 Owl inspired serrations . . . . . . . . . . . . . . . . . . 191

D.3 Combined leading and trailing edge serrations . . . . . . . . . . . . . . 192

D.3.1 Aerofoil configuration . . . . . . . . . . . . . . . . 193

D.3.2 Power spectra comparison . . . . . . . . . . . . . . . . . . 193 


\section{Declaration of Authorship}

I, Chaitanya PARUCHURI, declare that this thesis titled, "Aerofoil geometry effects on turbulence interaction noise" and the work presented in it are my own. I confirm that:

- This work was done wholly or mainly while in candidature for a research degree at this University.

- Where any part of this thesis has previously been submitted for a degree or any other qualification at this University or any other institution, this has been clearly stated.

- Where I have consulted the published work of others, this is always clearly attributed.

- Where I have quoted from the work of others, the source is always given. With the exception of such quotations, this thesis is entirely my own work.

- I have acknowledged all main sources of help.

- Where the thesis is based on work done by myself jointly with others, I have made clear exactly what was done by others and what I have contributed myself.

- Parts of this work have been published and the list has been indicated in chapter 1 of the thesis.

Signed:

Date: 



\section{Acknowledgements}

First and foremost, I would like to express my deep gratitude to my supervisor Phil Joseph for his invaluable support and his constant availability to discuss new ideas. I m very fortunate to have you as my supervisor, who cared not only about my progress in research but also cared immensely about my social and personal life. In this association with you for 3 years, I grew to be a better person both personally and academically. I really enjoyed every bit of my PhD. Thank you very much for everything and especially with all your patience in correcting my English.

I have also received much help and guidance from John Coupland for running CFD predictions. Thank you very much for your insights on the subject and it has immensely helped me to grow as a practical researcher.

I would also like to thank Gwenael Gabard for his valuable suggestions, Jae-Wook Kim and Sam Sinayoko for their valuable feedback during my review meetings, Oksana Stalnov for her constant motivation and support all along, and Narayanan Subramanian for his collaboration, teaching and helping me all along my PhD. I wish to thank Christina and Bharath from AFM group for PIV collaboration, Thomas Hainaut, Jacob Turner, and James Gill for their valuable time to discuss their simulation results.

I would like to thank Sue Brindle for helping me with all administrative paper-work and issues. I also want to thank Phil Oxborrow, Mike Street and Richard Dooler for their very useful insights into the design and manufacturing of most of the models used in this thesis and for responding so fast to last minute requests! Thank you Chris Oliver, Steve White for the good laughs and helping me to setup my experimental rig.

I thank all my colleagues from ISVR especially Victor, Thanos, Nadi, Thomas, Jayanth, Anderson and Juan who are with me all along this PhD journey. I would like to thank all my housemates especially Shruti, Abu and Devasena for their wonderful time in Southampton. I thank all my friends especially Sandeep, Chandu, Srikanth, Adi, Vijay prakash, Vijay, Ashu and D Sri for being with me all through the up and downs in this phase of my life. Thank you Priyanka Murthy for pushing me and helping me with the application process for PhD. I am thankful to Yogitha and my grandparents for their constant love. Amma and dad! Thank you for all your love, sacrifices, patience and support. Thank you Prati for your love and putting up with me during most stressful phase of my $\mathrm{PhD}$ despite the distance, and for finding the right words to bring the motivation back.

Finally, I am grateful to all the passionate people who played a key role in contributing to humanity via the fascinating field of science. They have always been my inspiration to explore and contribute back to the field in any way I could. Hoping this work will benefit the environment and the people around me. 



\section{List of Abbreviations}
AFM Aerodynamics and Flight Mechanics
ANTC Airbus Noise Technology Centre
CAA Computational aeroacoustics
CFD Computational Fluid Dynamics
DNS Direct numerical simulation
EPNdB Effective perceived noise level in decibels
LAA Lighthill acoustic analogy
LBM Lattice Boltzmann method
LINSUB LINearised SUBsonic unsteady cascade theory
LE Leading Edge
LES Large eddy simulation
NACA National Advisory Committee for Aeronautics
OGV Oult Guide Vane
OASPL Overall sound pressure level
ODE Ordinary differential equation
PWL Sound Power Level
PCL Potential core length
PNL Perceived noise level
PIV Particle Image Velocimetry
RANS Reynolds-averaged Navier-Stokes
SPL Sound pressure level
URANS Unsteady Reynolds-averaged Navier-Stokes 



\section{List of Symbols}

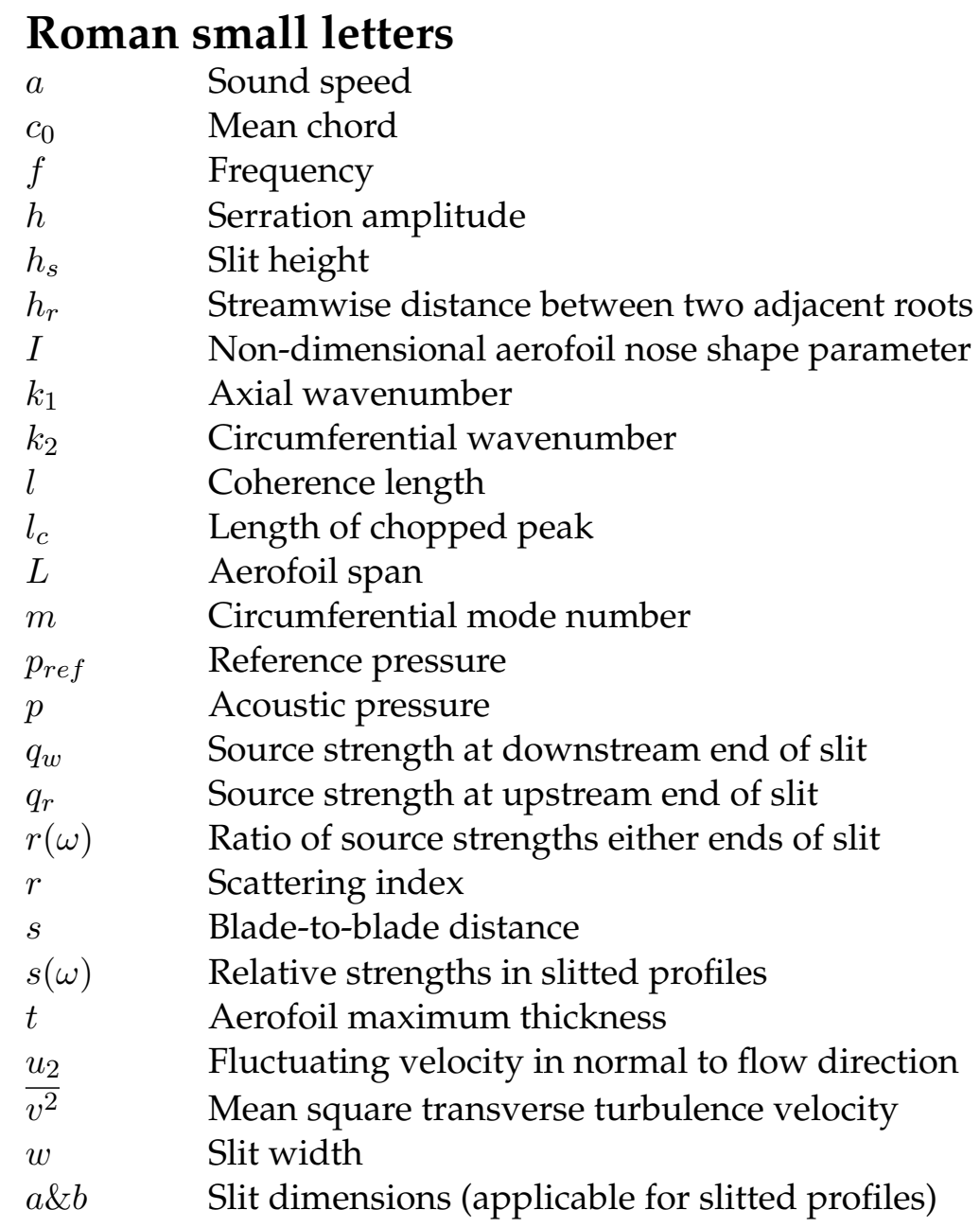

\section{Roman capital letters}

$B \quad$ Number of OGVs

$M \quad$ Mach number

$N_{r} \quad$ Number of valleys

$P \quad$ Total radiated acoustic power

$\triangle P W L \quad$ Sound power reductions

$P W L_{0} \quad$ Radiated sound power for flat plate

$\triangle P W L_{A} \quad$ Additional sound power reductions

$\triangle P W L_{I} \quad$ Sound power reductions due to interference of adjacent roots

$\triangle P W L_{S} \quad$ Sound power reductions for single-wavelength serrations

$P W L_{t} \quad$ Radiated sound power for aerofoil

$R \quad$ Radius of microphone arc

$R_{e} \quad$ Nose radius

$R_{r} \quad$ Non-dimensional amplitudes in cascade response

$S_{p p} \quad$ Acoustic pressure PSD 


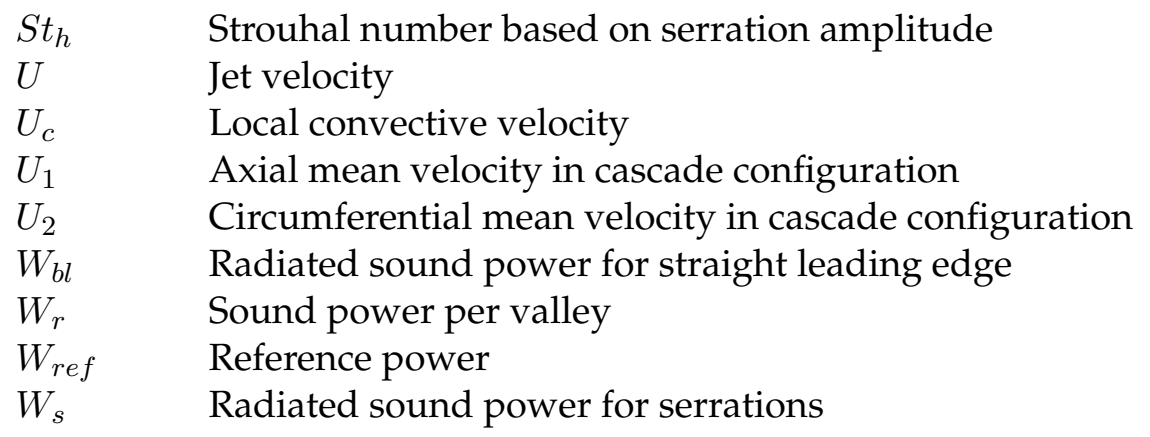

\section{Greek}

$\alpha_{r}$

$\beta$

$\beta_{r}$

$\eta$

$\epsilon$

$\gamma$

$\lambda$

$\lambda_{r}$

$\lambda_{0}$

$\Lambda$

$\Lambda_{t}$

$\mu_{h}$

$\mu_{a}$

$\phi$

$\rho$

$\theta$

$\theta_{L E}$

$\theta_{T E}$

$\omega$

$\Delta \omega_{3 d B}$

$\varpi$

Axial acoustic wavenumber

Non-dimensional factor for aerofoil thickness effects

Circumferential acoustic wavenumber

Non-dimensional constant of proportionality for serration

Small power ratio

Coherence function

Serration wavelength

Spanwise distance between two adjacent roots

Optimum wavelength of serration

Integral length scale in streamwise direction

Integral length scale in transverse direction

Non-dimensional hydrodynamic wavelength

Non-dimensional acoustic wavelength

Phase difference

Density of air

Incoming flow angle

Leading edge angle of blade

Trailing edge angle of blade

Frequency in $\mathrm{rad} / \mathrm{s}$

\section{Superscript}

Frequency bandwidth for $3 \mathrm{~dB}$ noise reduction

Sound power per unit length

$\pm \quad$ Upstream and Downstream wave 


\section{Chapter 1}

\section{Introduction}

\section{Contents}

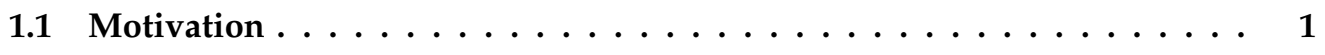

1.2 Turbulence-aerofoil interaction noise $\ldots \ldots \ldots \ldots \ldots$

1.2.1 Modeling of OGV response . . . . . . . . . . . . . 3

1.2 .2 Reduction of OGV response . . . . . . . . . . . . . . . . . 6

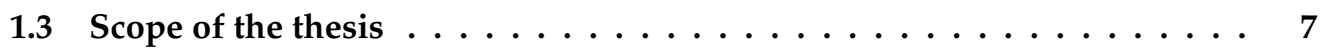

1.4 Original Contribution and Thesis structure $\ldots \ldots \ldots \ldots \ldots$

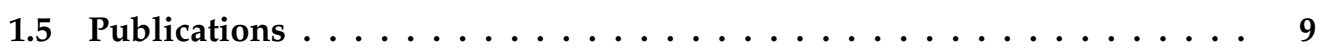

1.5 .1 Journal Papers $\ldots \ldots \ldots \ldots \ldots \ldots$

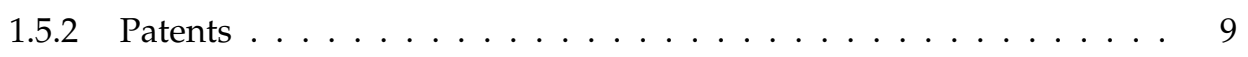

1.5.3 Conference Papers till April $2017 \ldots \ldots \ldots$. . . . . . . . . 10

\subsection{Motivation}

$\mathbf{F}$ lightpath 2050 has been drafted by the EUROPEAN COMMISSION to map out Europe's Vision for the future of civil aviation. Based on Flightpath 2050, the goal has been set to reduce the perceived noise emission from aircraft by $65 \%$ by 2050 . This poses a challenge in finding new methods for noise reduction on aircraft. Low-noise engines planned for the future, such as ultra-high-bypass-ratio engines and low-speed fans, will be effective in reducing jet noise, but will have little impact on fan noise. Moreover, the short ducts associated with these higher bypass ratio engines means less area for locating sound absorbing liners. Fan is now the dominant source of noise on a turbofan engine at approach condition. The Figure 1.1 taken from Astley et al. (2007) shows the relative noise contribution of the major components of a modern engine, during take-off and approach condition. Fan noise can be observed as important as jet noise at takeoff and clearly dominant at approach. 


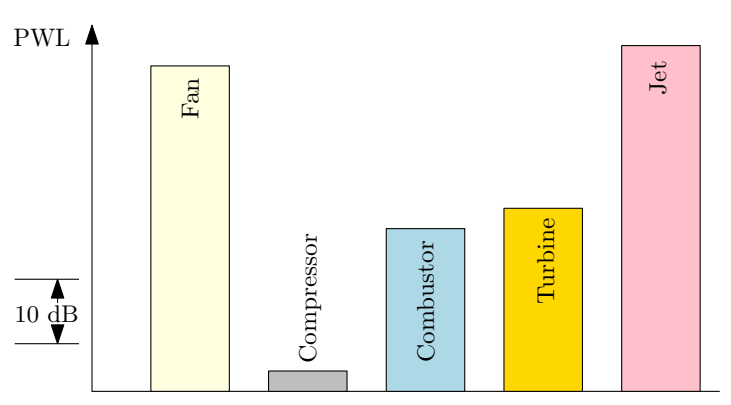

Takeoff

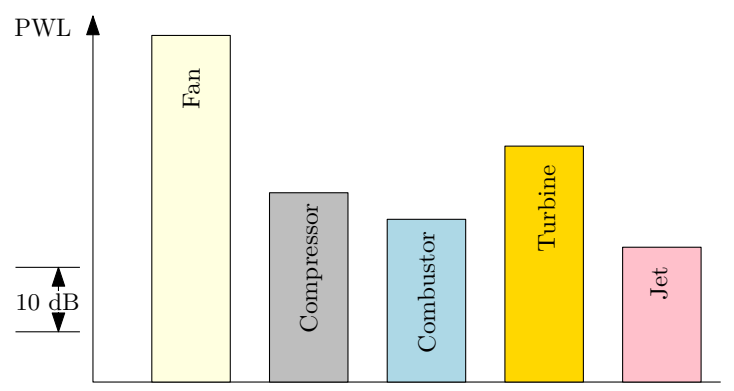

Approach

FIGURE 1.1: Relative power levels of noise sources in modern aircraft engines at take-off and approach. Data taken from Astley et al. (2007).

Fan noise can be decomposed into tonal noise and broadband noise components. At subsonic fan tip speeds the fan tones are produced by the interaction between between the mean wakes from the rotor and the downstream Outlet Guide Vanes (OGV's). The fan broadband noise is predominantly produced by the interaction of the turbulence in the rotor wakes with the OGV's. Other broadband noise generation mechanisms arise from turbulent eddies in the boundary layer passing over the rotor blades and scattering with the rotor trailing edge, interaction of the rotor blade tip vortex with the boundary layer at the inlet duct. Figure 1.2 is a schematic depicting these broadband noise sources.

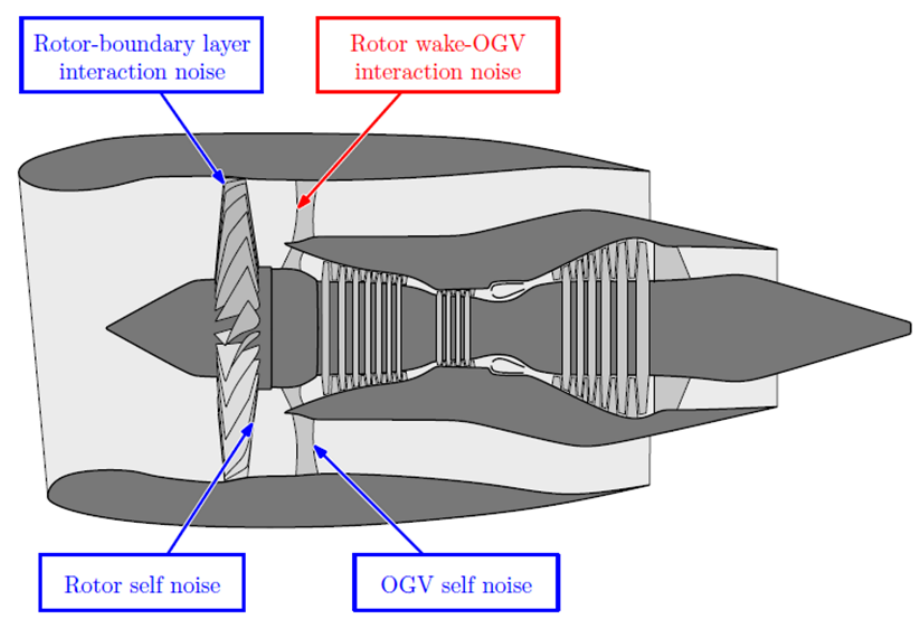

FIGURE 1.2: Noise generation mechanism of the interaction noise between the rotor wakes and the stator. Taken from Jenkins (2013).

The broadband noise generated through the interaction between the rotor wake and OGV is thought to be the dominant broadband fan noise mechanism. There is therefore an urgent need to understand and control this noise generation mechanism. At the present time it is not possible to predict accurately this noise source using analytical or computational methods, most probably because of the difficulty with accurately predicting rotor wake turbulence. In this thesis we provide evidence that the interaction of turbulence with the OGV's can be predicted with reasonable accuracy using flat plate analytic solutions or numerical solutions to the Euler equations. 


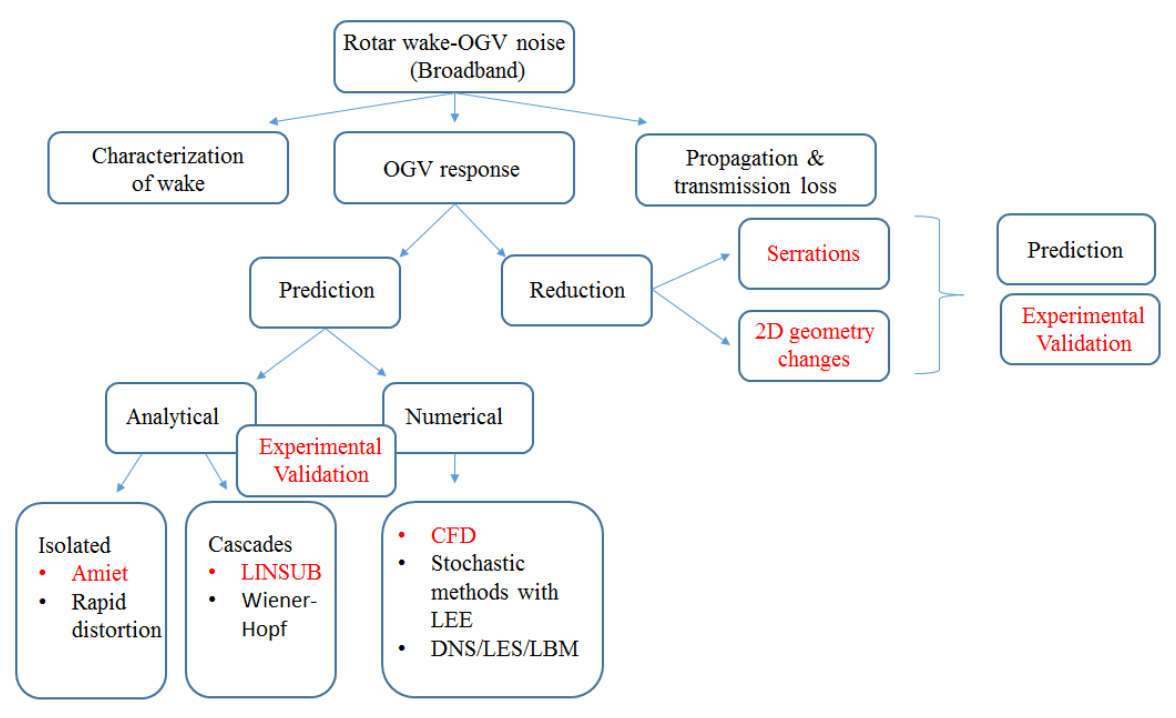

FIGURE 1.3: Flowchart of the available methods.

\subsection{Turbulence-aerofoil interaction noise}

The process of predicting rotor wake-OGV broadband interaction noise can be divided into three parts as indicated in figure 1.3: 1) Characterization of the turbulent wake, including velocity spectrum and two-point statistics; 2) Modeling the OGV response to a specified vortical input; 3) Prediction of the transmission loss of sound across the rotor to predict intake noise and the OGV to predict the noise from the bypass duct. The current thesis is focused on modeling of OGV response and its reduction by use of serrated leading edge geometries.

\subsubsection{Modeling of OGV response}

When an aerofoil is subjected to incoming turbulence, unsteady lift fluctuations are induced on the aerofoil surface, which radiates as a dipole distribution to the far-field.

\section{Analytical \& Experimental studies}

Early work on the prediction of broadband interaction noise approximating the aerofoil as single isolated flat plates (Amiet (1975)), or series of flat plates in a cascades (Whitehead (1987)). A review of the important analysis and experimental work on isolated aerofoils and aerofoil cascades is presented below.

\section{Interaction noise due to isolated aerofoils}

Sears (1941) was the first to develop a model to predict the unsteady lift, and hence the radiated noise, due to a flat plate interacting with a harmonic vortical gust. His model was restricted to a $2 \mathrm{D}$ gust and incompressible flow. Others have extended this theory to include the effects of obliqueness and fluid compressibility. The general three-dimensional compressible problem cannot be solved analytically but Graham (1970) deduced that the formulation for a three-dimensional gust in a compressible flow can be expressed in terms of the $2 \mathrm{D}$ compressible and the $3 \mathrm{D}$ incompressible results through the use of the so-called 
similarity conditions. Incident gusts are found to be either subcritical, with a subsonic phase speed along the leading edge of the blade, or supercritical, where the phase speed is supersonic along the blade leading edge.

Adamczyk (1974) took an approximate approach to the three-dimensional compressible flow problem and derived a closed-form expression for the aerodynamic response of an infinite swept flat-plate aerofoil in the form of a sum of two terms, representing the leading edge interaction and the subsequent trailing edge back-scattering.

One of the most widely used formulations of flat plate interaction noise is by Amiet (1975) to predict the noise over the flat plate interacting with 3D turbulence convecting in a compressible fluid. Extending the Graham (1970) and Adamczyk (1974) work, Amiet (1974) proposed iterative approach by using Schwarzschild theorem (Schwarzschild (1902)) to solve the Helmholtz equation. A good comparison with experiments has been shown in the frequency range where the aerofoil can be considered as non-compact. This thesis will provide careful experimental confirmation of this flat plate prediction. Recently, Santana et al. (2016a) has extended the Amiet's theory to the frequency regime where the aerofoil is considered as a compact noise source. Numerical corrections are developed to extend the validity of the Amiet theory to the low frequency regime (Billson (2002)).

Semi-analytical models have been developed to predict turbulence-aerofoil interaction noise for realistic aerofoils, that includes the effects of thickness Gershfeld (2004); Roger (2010); Glegg and Devenport (2010); Moriarty et al. (2005); Lysak et al. (2013); Billson (2002). This work will be discussed in section 2.1 and compared against careful measured noise data. The present thesis aims to better identify the regimes of validity of these analytical models by comparison with the experimental noise data.

\section{Interaction noise due to aerofoil cascades}

In a typical turbofan engine the OGV has vanes that are closely spaced with chords that are long relative to the gaps separating them. Acoustic interactions between the vanes is therefore a significant factor in the acoustic response to incident turbulence. The single aerofoil approach clearly cannot account for interactions between the vanes of an OGV across the full range of frequencies of interest.

In 1958 a two-dimensional unwrapped blade row was considered by Lane and Friedman (1958) in an analysis of the unsteady lift and moment on compressor and turbine blades for the prediction of flutter rather than noise. Torsional flutter was also the subject of work by Whitehead (1965) where a chord-wise distribution of bounded vorticity is described on the blade surfaces. The reaction of the bounded vorticity and their shed vortices are combined to form a description of the unsteady blade forces.

Kaji and Okazaki (1972) developed a model for the tonal noise generated due to the potential and velocity deficit interactions between a stator row and downstream rotor. Their formulation replaced the blade row with a distribution of pressure doublets, the strength of which were determined by the numerical solution of an integral equation.

Smith (1972) used a bounded vorticity approach, similar to that adopted in Whitehead (1987), in order to obtain an integral equation for the vorticity distribution along the chord of an infinite rectilinear cascade of unloaded flat plates due to a single frequency, harmonic vortical gust. This source term was then used to compute the upstream and downstream acoustic pressures. Solutions to the integral equation were obtained using a collocation technique. Further details of this theory and a FORTAN code, LINSUB, to compute the cascade response based on this formulation were presented in Whitehead (1987). 
The analyses of Kaji and Okazaki (1972), Smith (1972), Whitehead (1987) mentioned above all employ numerical schemes in order to obtain their solutions, introducing significant computational difficulties at high frequencies. Approximate analytic expressions for the sound transmission through a two-dimensional cascade were presented by Mani and Horvay (1970). Their approach was based on the Wiener-Hopf technique and neglected interaction between the leading and trailing edges by using semi-infinite blades for both the downstream-propagating waves from the leading edges and upstream-propagating waves from the trailing edge. These wave interactions were resolved in the vane overlap region, thus limiting this technique to overlapping configurations.

Peake and Kerschen (1997) have formulated the derivation of a consistent asymptotic approximation for the upstream acoustic radiation generated by the interaction between convected disturbances and a cascade of loaded flat-plate aerofoils. At the high frequencies considered by Peake and Kerschen (1997), the noise is generated in small regions around the blade leading edges, and is then diffracted by the leading edges of all the other blades in the cascade, reflected by the adjacent blades, and refracted as it propagates through the non-uniform flow. Hanson and Horan (1998) were the first to develop a theory for the turbulence-cascade interaction noise, giving the sound power spectrum in terms of the 3 dimensional wavenumber spectrum of the turbulence using the 3D harmonic cascade response function developed by Glegg $(1998,1999)$. They showed the noise variation with the Mach number were different on the upstream and downstream of the cascade. They also showed that the noise is proportional to vane count but the dependence on the chord length is negligible. Noise sensitivity with the integral length scale of turbulence is also discussed.

Evers and Peake (2002) extended the work of Peake and Kerschen (1997) to calculate the upstream radiated noise due to the interaction between a single vortical gust and a cascade of flat plates at non-zero angle of attack. They also included the effects of camber and thickness in their analysis. They showed that the blade geometry effects on the radiated power are significant in case of interaction with a single gust, whereas in the case of turbulence, which comprises a continuum of oblique gust components, aerofoil geometry effects on a cascade are within $2 \mathrm{~dB}$ compared to a flat plate. This implies the blade geometry can have a significant effect on the tonal noise components generated by rotorstator interaction (i.e. by single harmonic gusts), but that the broadband part of the noise spectrum is relatively unaffected (Evers and Peake (2002)).

In the current thesis, the aerofoil geometry effects on turbulence-cascade interaction noise has been investigated using CFD.

\section{Numerical studies of aerofoil interaction noise}

Numerical methods, such as DNS/LES/LBM, are computationally prohibitively expensive to solve for complete 3D domains of rotor wake-OGV interaction. Hybrid approaches are therefore adopted in which incompressible flow solvers are used to compute the acoustic source terms and a form of the acoustic analogy then used to propagate the solution.

To predict broadband noise up a specific frequency, it is necessary to predict all appropriate turbulence length scales from solutions of the Navier-Stokes equations. A different pragmatic approach is to artificially generate a turbulence velocity field superimposed on a mean flow solution that matches some assumed turbulence description such as frequency spectra or two point correlations. The acoustic field due to interaction noise, which is essentially an inviscid phenomenon, can then be computed from solutions of the Euler equations. Examples of the synthetic approach include the Random Particle-Mesh method (Ewert (2008), 
Gea-Aguilera et al. (2015)), Random-Vortex-Particle method (Dieste (2011)) and the Fourier method (Gill et al. (2013)).

Earlier studies aimed at predicting the the noise due to interaction between aerofoils and vortical disturbances have considered single frequency harmonic sinusoidal gusts. Atassi et al. (1990) used a Computational Aero-acoustics (CAA) approach to investigate the effects of thickness on the noise due to a harmonic gust, and observed a reduction in the noise at high frequency due to the effect of aerofoil thickness compared to a flat plate. the effect was greatest in the downstream observer direction. Lockard and Morris (1998) also investigated computationally the effects of aerofoil thickness in both inviscid and viscous simulations, and arrived at similar conclusions to those of Atassi et al. (1990). Gill et al. (2013) used CAA to investigate the effects of both aerofoil thickness and leading edge radius on the noise due to a range of NACA 4-digit aerofoils, and also predicted noise reductions when either, or both, parameters were increased. However, the noise reductions due to leading edge nose radius were found to be smaller, than those of thickness, and were found to mostly affect the noise at high frequencies.

More recently, Polacsek et al. (2015) presented a three-dimensional computational hybrid method which is a time-domain Euler solver coupled to a synthetic turbulence model with suited inflow boundary condition. It is aimed at predicting the acoustic modes in a ducted annular cascade subjected to a homogeneous isotropic turbulent flow. The predicted fluctuating pressures over the aerofoil were used as the input to a Ffowcs Williams and Hawkings integral solver to calculate the radiated sound field. However, the turbulence description is restricted to parallel gusts (setting the spanwise wave number equal to zero).

In this thesis, we adopt a slightly different approach for computing broadband interaction noise due to a 2D aerofoil cascade interacting with 2D isotropic turbulence. The mean flow is predicted from the RANS solution with the turbulence represented by a summation of Fourier gusts compatible with the periodicity of the duct. The upstream and downstream pressure amplitudes generated by the cascade due to each gust is computed from the solutions of the Unsteady RANS (URANS) solver in the frequency domain. The unsteady calculations are performed for a single blade passage but with the phase delay of $2 \pi \mathrm{m} / B$, introduced between the upper and lower boundaries to simulate the full annulus, where $m$ is the transverse mode number and $B$ is the number of vanes.

\subsubsection{Reduction of OGV response}

One approach to reducing rotor wake-OGV interaction noise is by the addition of serrations on the Leading Edge (LE) of the OGV's. These LE serrations can be found on owl wings and whale flippers, either to reduce noise or/and enhance hydro and aero-dynamic performance. It has been established from last 10 years that introducing LE serrations on aerofoils can improve their aerodynamic performance at post-stall conditions Skillen et al. (2014); Zhang et al. (2013); Hansen et al. (2011); Yoon et al. (2011); Johari et al. (2007). Collins (1981) has observed that the presence of leading edge serrations on wings can improve the lowspeed lift and stall performance of aircraft during take-off and landing. Bachmann et al. (2007) showed that the barn owl exhibits 'silent' flight due to serrations at the leading edge of the wing and the fringes at the edges of each feather. They proposed that the topographies and mechanisms underlying this silent flight might eventually be employed for aerodynamic purposes thus resulting in new wing designs in modern aircraft. They showed that the owl is quieter than the pigeon due to the presence of serrations at its leading edge and the fringes at the edges of each quill. 
This thesis provides a detailed investigation on the aeroacoustic performance of aerofoils with leading edge serrations. Visualisation of the flow around the serrated aerofoils was undertaken using Particle Image Velocimetry (PIV) techniques. This thesis also presents a detailed parametric study on the noise due to single-wavelength LE serrations applied to both flat plates and realistic aerofoils. Chapter 4 presents a more complete review of the previous literature on this subject.

\subsection{Scope of the thesis}

The aims of this thesis are four-fold:

1. To experimentally assess aerofoil geometry effects, such as aerofoil thickness, nose radius and camber, on turbulence-aerofoil interaction noise.

2. To develop a numerical method using CFD to predict broadband interaction noise on a 2D cascade of realistic aerofoils interacting with 2D turbulence.

3. To provide a detailed systematic experimental study into the noise reduction mechanism and performance of single-wavelength serrated leading edge aerofoils of the type investigated previously by other researchers.

4. To develop innovative leading edge designs that provide superior noise reductions compared to conventional single-wavelength sinusoidal leading-edge serrations.

Figure 1.3 shows the components of work required to predict and reduce rotor wake-OGV interaction noise. This thesis deals with the components marked in red.

\subsection{Original Contribution and Thesis structure}

The main contributions of the thesis are listed below. Each one relates directly to a thesis chapter.

1. Chapter 2: A detailed experimental study has been performed to understand the influence of aerofoil geometry on turbulence - aerofoil interaction noise on isolated aerofoils. Systematic noise measurements have been carried out by varying aerofoil thickness and leading edge nose radius separately. The flat plate analytical theory has been shown to be in very close agreement with measurements of the radiated noise from flat plate interacting with grid-generated turbulence. It is shown that local leading edge geometry, such as leading nose radius, is an important parameter in determining noise radiation from 'thin' aerofoils at high frequencies $f t / U>0.2$, particularly when the gust wavelength is comparable to the nose radius. The thesis makes clear that for thin aerofoils broadband interaction noise cannot be predicted based on a non-dimensional frequency based on a single length-scale parameter related to the blade geometry, unlike for thick aerofoils where good collapse of the noise spectra is obtained for $f t / U$. All measured and predicted noise data exhibit a dependence on velocity that varies as $U / f$. No difference in behaviour is observed between the predicted noise at low Mach numbers $(M \leq 0.3)$ and high Mach numbers $(M \geq 0.3)$ suggesting that compressibility effects are not an issue in interaction noise problems. Noise measurement at low Mach numbers can therefore be extrapolated to higher speeds. Appendix A presents a detailed description of the experimental facility and measurement techniques implemented to perform noise and aerodynamic measurements presented in this thesis. 
Appendix B also presents the 3D Amiet's formulation along with a discussion into the significance of sub-critical and super-critical gust incidence on radiated noise.

2. Chapter 3: A procedure to predict the broadband noise generated due to the interaction of 2D isotropic turbulence with a cascade of 2-dimensional realistic aerofoils is developed. The prediction is based on a Fourier synthesis of the $2 \mathrm{D}$ turbulence and computing the radiation from each component separately. A linearized Unsteady Navier-Stokes solver is used to predict the aerofoil response due to an incoming harmonic vortical gust. The influence of flow incidence, the mean flow, aerofoil thickness and aerofoil nose radius on turbulence-cascade interaction noise are discussed. The chapter demonstrate that broadband noise is only weakly affected by changes in aerofoil geometry for realistic mean flows and frequencies. This finding is consistent with the observation made for isolated aerofoils that $f t / U$ is the dominant factor in determining noise radiation and is relatively unaffected by other modifications to geometry such as nose radius and camber at the frequencies and flow speeds of interest at approach conditions. Appendix C presents the numerical setup for the CFD simulations, which includes some grid convergence studies.

3. Chapter 4: A detailed experimental study is performed into the sensitivity of serrations applied to the aerofoil leading edge. The study has investigated variations in serration parameters (amplitude and wavelength) and turbulence integral lengthscale. An optimum serration wavelength $\lambda_{0}$ is identified whereby maximum noise reductions are obtained. This optimum value corresponds to when the transverse integral length-scale of the turbulent flow is roughly one-forth the serration wavelength. One of the most important findings of this chapter is that at the optimum serration wavelength $\lambda_{0}$, the ratio of sound power radiation from the serrated aerofoil to the baseline (straight edge) aerofoil varies inversely proportional to the Strouhal number $S t_{h}=f h / U$, where $f, h$ and $U$ are frequency, serration amplitude and flow speed, respectively. A simple model is proposed to explain this behavior. These findings are then applied to realistic aerofoils. It was found that these 3D effects produced only a small influence on the noise reductions compared to a flat plate.

4. Chapter 4: The flow around the leading edge serration aerofoil was visualised using PIV techniques. This work was undertaken in collaboration with colleagues from the AFM group at the University of Southampton. The results of this study showed that the flow remains attached to the aerofoil, even close to the leading edge where relatively rapid changes in profile occur. These preliminary measurements suggests that lift and drag may be marginally reduced and increased respectively. This is consistent with the aerodynamic measurements performed on the same wind tunnel.

5. Chapter 5: A new leading edge serration profile is proposed comprising of the sum of two sinusoids. It has been shown that it is capable of providing substantially better noise reductions than the maximum noise reduction achievable on single-wavelength serration geometries of the same amplitude. A simple model is proposed to explain the noise reduction mechanism for these double wavelength serration profiles, which is shown to be in close agreement with the measured noise reduction.

6. Chapter 6: A new leading edge geometry is proposed comprising of slits cut into the roots of a sawtooth serration, which is designed to overcome the fundamental limitation of double-wavelength serrations whose performance was shown to be limited by the imperfect coherence between adjacent roots. A model has been developed to predict and understand the additional noise reductions due to slitted-root serration 
profiles which are shown to be a function of relative source strength between the dominant sources on either side of the slit. It has been demonstrated that even slits by themselves can provide greater levels of noise reduction than conventional profiles of the same amplitude.

7. Chapter 7, Appendix D: Two further leading edge profiles were proposed and investigated aimed at improving still further the noise reduction performance of singlewavelength leading edge serrations. These include variable slits, chopped-peak serrations, and 'random' leading edge profile. Investigation of the noise due to these geometries also provided an understanding into some fundamental issues relating to the noise reduction mechanism.

\subsection{Publications}

\subsubsection{Journal Papers}

1. P. Chaitanya, P. Joseph, S. Narayanan, C. Vanderwel, J Turner, J. W. Kim, and B. Ganapathisubramani, (2017) 'Performance and mechanism of sinusoidal leading edge serrations for the reduction of aerofoil interaction noise', vol. 818, pp. 435-464, Journal of Fluid Mechanics.

2. L. Ayton and P. Chaitanya, (2017) 'Analytical and experimental investigation into the effects of leading-edge radius on gust-aerofoil interaction noise', vol. 829, pp. 780-808, Journal of Fluid Mechanics.

3. S. Narayanan, P. Chaitanya, S. Haeri, P. Joseph, J. W. Kim, and C. Polacsek (2015). 'Airfoil noise reductions through leading edge serrations', 27 (2), 025109, Physics of Fluids.

4. O. Stalnov, P. Chaitanya and P. Joseph (2016). 'Towards a non-empirical trailing edge noise prediction model', 372, PP. 50-68, Journal of Sound and Vibration.

5. P. Chaitanya, P. Joseph, S. Narayanan and J. W. Kim, (2017) 'Aerofoil broadband noise reductions through double-wavelength leading edge serrations; a new control concept', submitted to Journal of Fluid Mechanics.

6. P. Chaitanya, P. Joseph, (2017) 'Slitted leading edge profiles for the reduction of turbulenceaerofoil interaction noise', submitted to Journal of Acoustical Society of America.

Under preparation

7. P. Chaitanya, P. Joseph, (2017) 'Influence of 2D geometry effects on turbulence interaction noise', AIAA Journal.

\subsubsection{Patents}

1. S. Narayanan, P. Chaitanya, P. Joseph, J. W. Kim, S. Haeri, Improved airfoil broadband reductions through double-frequency leading edge serrations, US20170022820, EP3121376A1. 
2. P. Chaitanya, P. Joseph, J. W. Kim, 'Improved aerofoil broadband noise reductions through the use of slitted root leading edge serrations', US20170241278, EP3208420 A1.

3. P. Chaitanya, P. Joseph, 'A novel design for enhanced turbulence aerofoil interaction noise reductions', (under consideration for a patent with Rolls-Royce - 16305).

4. P. Chaitanya, P. Joseph, ' Enhanced turbulence aerofoil interaction noise reduction', (under consideration for a patent with Rolls-Royce - 17531).

\subsubsection{Conference Papers till April 2017}

1. P. Chaitanya, J. Gill, S. Narayanan, P. Joseph, C. Vanderwel, X. Zhang and B. Ganapathisubramani. 'Aerofoil geometry effects on turbulence interaction noise', presented in 21st AIAA/CEAS conference, June 2015, Dallas, number 2015-2830.

2. P. Chaitanya, S. Narayanan, P. Joseph, C. Vanderwel, J. W. Kim, and B. Ganapathisubramani. 'Broadband noise reduction through leading edge serrations on realistic aerofoils' presented in 21st AIAA/CEAS conference, June 2015, Dallas, number 2015-2202.

3. P. Chaitanya, O. Stalnov, P. Joseph, R. Leung. 'On the robustness of the TNO model for aerofoil self-noise prediction', presented in 21st AIAA/CEAS conference, June 2015, Dallas, number 2015-2367.

4. O. Stalnov, P. Chaitanya, P. Joseph, 'Prediction of Broadband Trailing-Edge Noise Based on Blake Model and Amiet Theory', presented in 21st AIAA/CEAS conference, June 2015, Dallas, number .

5. P. Chaitanya, P. Joseph, 'A Novel Leading Edge Serrations for Low Frequency Broadband Attenuation', presented in 5th Scientific Workshop of the European X-Noise EV Network on Broadband Noise of Rotors and Airframes, La Rochelle, 2015.

6. P. Chaitanya, J Coupland, P. Joseph, 'Aerofoil geometry effects on turbulence interaction noise in cascades', presented in 22nd AIAA/CEAS conference, May 2016, Lyon, number 2016-2738.

7. P. Chaitanya, P. Joseph, S. Narayanan, J. W. Kim, 'Leading edge serration geometries for significantly enhanced leading edge noise reductions', presented in 22nd AIAA/CEAS conference, May 2016, Lyon, number 2016-2736.

8. S. Narayanan, P. Joseph, S. Haeri, J. W. Kim, P. Chaitanya, and C. Polacsek (2014). 'Noise reduction studies from the leading edge of serrated at plates'. presented in 20th AIAA/CEAS conference, number 2014-2320.

9. R. Leung, P. Chaitanya, P. Joseph, 'Effect of Aerofoil Thickness on Trailing Edge Noise', presented in 22nd AIAA/CEAS conference, May 2016, Lyon, number 2016-2815.

10. J. Turner, J. W. Kim, P. Chaitanya and P. Joseph, 'Towards Understanding Aerofoils with Dual-Frequency Wavy Leading Edges Interacting with Vortical Disturbances', presented in 22nd AIAA/CEAS conference, May 2016, Lyon, number 2016-2951.

11. A. Vathylakis, T. P. Chong, P. Chaitanya and P. F. Joseph, 'Sensitivity of Aerofoil Self Noise Reductions to Serration Flap Angles', presented in 22nd AIAA/CEAS conference, May 2016, Lyon, number 2016-2837. 


\title{
Chapter 2
}

\section{Turbulence-aerofoil interaction (TAI) noise}

\author{
Contents

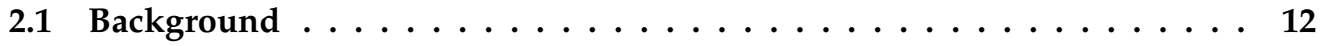

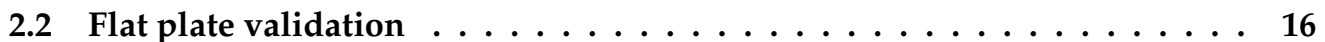 \\ 2.3 Aerofoil Geometry effects . . . . . . . . . . . . . . . . 20 \\ 2.3 .1 Aerofoil Configurations . . . . . . . . . . . . . . . . 20 \\ 2.3 .2 Influence of Self-Noise . . . . . . . . . . . . . . . . . . . 21 \\ 2.3.3 Effect on interaction noise due to thickness . . . . . . . . . . . 22 \\ 2.3.4 Comparison of thickness-based empirical models for the prediction \\ on interaction from aerofoils . . . . . . . . . . . . . . 24 \\ 2.3.5 Effect on interaction noise due to leading edge nose radius . . . . . 27 \\ 2.3.6 Discussion on thickness and nose radius effects . . . . . . . . . . 29 \\ 2.3.7 Effect of camber on turbulence-aerofoil interaction noise . . . . . . . 29 \\ 2.3.8 Effect of AOA on turbulence-aerofoil interaction noise . . . . . . . 30 \\ 2.4 Effects on interaction noise due to flow speed . . . . . . . . . . . 31 \\ 2.5 Comparisons with CAA Simulations $\ldots \ldots \ldots \ldots \ldots \ldots$

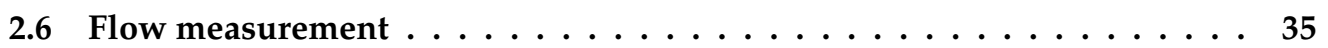

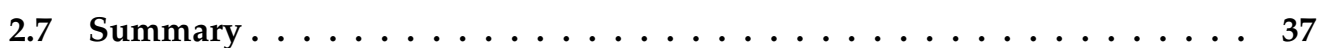

This chapter provides a detailed experimental study aimed at understanding the influence of geometry on isolated aerofoils on turbulence - aerofoil broadband interaction noise. The main contributions of this chapter are as follows:

1. The effects of thickness and leading edge nose radius on the noise are quantified experimentally and plotted. Collapse of the noise spectra for various aerofoils thicknesses are attempted by plotting against non-dimensional frequency $(f t / U)$, where $t$ is the maximum thickness of the aerofoil. This results of this chapter makes clear that there does not exists single length-scale parameter associated with the aerofoil geometry for 'thin' aerofoils that allows interaction noise spectra to be collapsed and that it is a complex function of the complete aerofoil geometry. This is in contrast to thick aerofoils where good collapse of the noise spectra is obtained by plotted against $f t / U$.

2. The effect of camber and angle of attack on broadband interaction noise is investigated.

3. Attempts are made to explain the mechanism of noise reduction through measurements of the flow behaviour around the aerofoil, particularly in the vicinity of the stagnation region. 
4. Measurements of interaction noise for various aerofoil geometries are compared against the results from a high-order linearized Euler CAA solver, where agreement to within $1 \mathrm{~dB}$ is obtained over a range of low Mach numbers $M \leq 0.3$. However, the computations were performed at much higher Mach number where no change in behaviour was predicted due to compressibility effects, suggesting that low Mach number measurements may be extrapolated to higher flow speeds.

\subsection{Background}

A number of researchers have shown that aerofoil geometry can affect turbulence-aerofoil interaction noise at high frequencies. Paterson and Amiet (1977) made careful measurements of the broadband interaction noise due to a NACA 0012 aerofoil and compared the results against the flat plate noise model due to Amiet (1975) at different flow speeds up to $165 \mathrm{~m} / \mathrm{s}$. It was observed that a deviation from the flat plate analytical model occurs at high frequencies corresponding to gust wavelengths $U / f$ that are smaller than the aerofoil thickness. They not only found that the unsteady surface pressure strongly peaked towards the leading edge, but also that other chord-wise positions also directly contribute to the far-field noise. They also showed that the effect of angle of attack on the radiated noise is small.

Olsen and Wagner (1982) investigated experimentally the effect of aerofoil thickness on broadband interaction noise by varying the maximum thickness-to-chord ratio between $3 \%$, $13 \%$ and $37 \%$ on a NACA symmetric aerofoils. Two main observations were made: The radiated sound at a fixed frequency reduces with increasing thickness, and this decrease in noise due to thickness increases with increasing frequency.

The problem of aerfoil-turbulence interaction noise has also been studied analytically. Goldstein and Atassi (1976) applied rapid gust distortion theory to investigate the effect of thickness on the radiated noise. The theory analyzes the interaction between a periodic twodimensional gust with an aerofoil in uniform flow and shows that the oncoming gust is distorted by the steady potential flow field about the aerofoil. They concluded that the mean flow streamlines around the aerofoil distorts the incoming gusts that mostly affects the radiated noise at high frequencies. This theory was later extended by Atassi (1984) to small camber aerofoils placed at small angle of attack. Atassi (1984) showed that for a thin aerofoil with small camber, placed at small angle of attack to a potential mean flow and subject to a periodic gust, the unsteady lift caused by the gust can be constructed by linear superposition to the Sears lift of three independent components accounting separately for the effects of aerofoil thickness, aerofoil camber and non-zero angle of attack of the mean flow. Atassi (1984) found that capturing the thickness effects was difficult due to the asymptotic nature of the stagnation region around the nose radius. They found that the noise generation is concentrated in the local leading-edge region.

Tsai (1992) used similar rapid distortion equations to develop a closed-form asymptotic solution for noise generated by a convecting single gust interacting with a thin, two-dimensional, symmetric aerofoil. The influence of aerofoil thickness on the total sound power is found to be controlled primarily by the Strouhal number $f R_{e} / U$, where $R_{e}$ is the nose radius of the aerofoil. Goldstein and Atassi (1976) and Atassi (1984) model was later extended to include the effects of angle of attack and camber (Myers and Kerschen $(1995,1997)$ ) which is based on linearization of the Euler equations about the steady subsonic flow past the aerofoil. Parametric calculations presented by Myers and Kerschen $(1995,1997)$ illustrate both incidence angle and moderate amounts of aerofoil camber can significantly affect the sound 
field produced by aerofoil gust interactions. All these analytic work is based on single gust approach.

An alternative to single gust-based methods is to perform the noise calculations in the time domain, which can more easily incorporate the complex motion of vortices close to the leading edge, which cannot otherwise be captured in the frequency domain. Grace (2001) has used a panel method to capture the aerofoil thickness effects on interaction noise. It was shown that the unsteady blade response decreases with increasing thickness, which is demonstrated on a NACA 0012 aerofoil. The time domain models were compared with a gust model using rapid distortion theory on a Joukowski aerofoil, where the gust model required multiple longitudinal wave number response calculations in order to model the asymmetry. The results of these time domain approaches was found to depend on the selection of streamlines around the aerofoil and also on the location of the vortex point. A sensitivity study on the location of the vortex point has been performed by Glegg and Devenport (2009). To overcome the streamline dependence on the noise prediction, they superimposed the line of vortices to model a gust with a step discontinuity. It was shown that the unsteady lift peaks when the vortex passes the leading edge, but if the vortex is close to the leading edge response is reduced. Increasing aerofoil thickness was found to move the location of the unsteady lift peak downstream, and reduce its magnitude.

Glegg and Devenport (2010) later extended their work from Joukowski aerofoils to arbitrary aerofoils using panel methods. Two different approaches were used: One using a conventional vortex panel method in which the Biot-Savart law is used to solve for the flow velocity, and the other expressing the velocity potential in terms of stagnation enthalpy. Glegg and Devenport showed that the panel method produces noise predictions up to a reduced frequency $(\omega b / U)$ of 40 . This method captures the high frequency reduction associated with thicker aerofoils and also shows that the radiated sound is weakly dependent on the angle of attack. This panel method has been used on various aerofoils and has been compared to experimental measurements by Devenport et al. (2010). Three different aerofoils of varying chord, thickness and camber were introduced in a turbulent flow and the radiated noise compared with panel method solutions. It was shown that the angle of attack has a strong effect on the noise for a Fourier component of turbulence, but this effect considerably weakens when summed over all gust components in the isotropic turbulence velocity spectrum. It was concluded that the effect on noise due to angle of attack are significant for non-isotropic turbulence and are dependent on aerofoil shape. Camber effects were observed to be small in Devenport et al. (2010)s study. Similar observations by Santana et al. (2012) have been made, which uses a panel method to predict the turbulence interaction noise.

Kucukcoskun et al. (2013) has also developed a semi-analytical model, based on Amiet's theory, to predict turbulence-interaction noise. This method incorporates an additional geometric near-field correction. This is followed by an implementation of a strip method to account for spanwise variations of the incoming flow. Finally, uses a boundary element method approach to compute the scattered acoustic field of the aerofoil. This method has also been validated experimentally with reasonable agreement.

A time-domain analytical method based on the vortex lift theory of Howe (2001) has been developed by Lysak et al. (2013); Lysak (2011), to capture the effects of aerofoil thickness on the high frequency aerofoil response to an impinging vortical gust. This approach can account for gust distortion by the mean flow around the aerofoil leading edge. The unsteady lift can be calculated by evaluating the potential field along the drift lines of the mean flowfield around the aerofoil. A simple correction factor to account for the effect of thickness in the turbulent gust model was developed for the NACA 65 series aerofoils. This gust 
response correction factor was compared with other models available in the literature, where a reasonable match was obtained between them.

The numerical models outlined above are generally based on complex numerical prediction schemes that provide little insight into the dependence on broadband interaction noise due to aerofoil geometry effects. There is therefore a need to develop more simplified, physicsbased models that predict the effects of aerofoil geometry on noise. Moreau et al. (2005) developed a semi-empirical correction factor that can be applied to the flat plate noise predictions to predict the noise from real aerofoils. To capture aerofoil geometry effects, such as camber and thickness, an additional correction term was used in the radiation integral of Amiet's method Amiet (1975). In addition to these changes, the input turbulence spectra, based on Hunt's rapid distortion theory, which can capture the high frequency roll-off, was implemented. These corrections were validated against measured experimental NACA 0012 noise data. This study was extended to formulate the relation between the noise reduction due to thickness effects, which is a function of the relative thickness and inversely proportional to the ratio of turbulent length scale and the flow speed Roger (2010). The method was compared with existing experimental data (Paterson and Amiet (1977)) and a reasonable collapse was observed when plotted against $f c_{0} / U$, where $c_{0}$ is the aerofoil chord. The direct influence of the integral length scale was not investigated explicitly.

Another approach to providing empirical factors to include real geometry effects on interaction noise has been taken by Gershfeld (2004). Howe's Green's function which was originally developed for trailing edge noise, has been used to accounts for the effects on noise due to aerofoil thickness. This was used to develop the correction factor $e^{-\omega t / 2 U}$ to predict the difference in noise between an aerofoil with thickness $t$ and a flat plate. This correction factor was shown to be in close agreement with the experimental data of Paterson and Amiet (1977) measured on the comparatively thick NACA0012 aerofoil. However, the details of this derivation were not provided. In this chapter we show that this correction factor is reasonably valid for thick aerofoil but breaks down for thin aerofoil. More importantly, it appears to provide an upper bound estimate for the noise reduction compared to a flat plate.

Moriarty et al. (2005) who derived a numerically-based correction factor to account for aerofoil thickness effects on noise based on a boundary element method. The noise reduction factor due to aerofoil geometry was also developed, based on a combination of the thickness to chord ratio at positions of $1 \%$ and $10 \%$ along the chord from the leading edge. This empirical noise expression for the noise reduction compared to a flat plate was developed from the noise data of 7 different configurations of camber, nose radius and maximum thickness. The model was validated against 6 new aerofoils, whose geometries were similar aerofoils used to develop the model.

Oerlemans and Migliore (2004) also measured the noise radiation from aerofoils in turbulent flows. They observed that aerofoils with a blunter leading edge nose profile produce less interaction noise. They also found that the inflow turbulence noise level scales with $U^{6}$. Hall et al. (2011) have also undertaken interaction noise measurements in which the first $10 \%$ of the aerofoil shape near the leading edge was altered but maintaining the maximum thickness location and camber. Again, it was observed that aerofoils with larger nose radii generate lower radiation noise. The maximum noise reduction due to this change was observed to occur at frequencies where the reduced frequency, based on leading edge thickness, is of the order unity.

Recently, Santana et al. (2016a) discusses a limitation of Amiet's theory which restricts its capability of predicting the noise predictions at frequencies where the aerofoil is considered 
a compact noise source where the acoustic wavelength is comparable to the aerofoil chord $\left(0.5 \leq \omega c_{0} / a \leq 1\right)$. To overcome this limitation Santana et al. (2016a) revisits Amiet's approach and uses Schwarzschild's technique in an iterative procedure where the second calculation can only be computed in terms of approximate analytical expression. He showed after two iterations Amiet's noise predictions are precise at high-frequencies, however generally overestimated in the frequency range where the aerofoil is considered a compact noise source (Santana et al. (2016a)). In the current thesis, at these low frequencies, the jet noise masks the total noise in the measurements. Thus, Amiet's theory Amiet (1975) has been used to compared against flat plate measurements.

Santana et al. (2016b) proposes a modification to the von Krmn model for isotropic turbulence, inspired by asymptotic results of Rapid Distortion Theory, and supported by detailed hot wire anemometry and stereo-PIV measurements performed in a plane upstream of a NACA-0012 aerofoil subjected to incoming grid turbulence. It was shown at a distance close to 12 percent of the aerofoil leading-edge curvature radius has important turbulence distortion effects, affecting both the spatial correlation and amplitude of the turbulent velocity components. Measuring the integral longitudinal correlation length at that fixed location, far-field radiated noise is predicted by using the modified turbulence spectra and compared against the measurements. Here the flat plate response function is considered to predict the far-field radiated noise for NACA-0012 aerofoil. But the universality of this model for different aerofoil nose shape profiles and thicknesses has to be validated.

More recent analytic (Ayton (2016)), numerical (Hainaut et al. (2016)) and experimental studies (Chaitanya et al. (2015a)) were conducted to understand the influence of nose radius, thickness, maximum thickness location, chord etc on radiated noise. The summary of the results are as follows:

1. Small changes in the profile of the leading edge, nose radius, maximum thickness, etc have a significant effect on gust distortion in the vicinity of the nose radius, which in turn effects the radiated noise.

2. Factors such as thickness, nose radius and position of maximum thickness are, by them-selves, not sufficient to fully account for the distortion of the turbulence for the prediction of radiated noise.

3. The analytic solution for the leading-edge inner region is a complex function (Ayton (2016)) of the complete nose profile. Further work is required to simplify these thickness correction terms in analytic solution and develop an elegant correction term that captures the changes in aerofoil geometry.

The conclusions of the numerical and analytical study are similar to experimental observations. Essentially, we need to account the complete aerofoil profile until the position of maximum thickness to capture the gust distortion to predict the radiated noise accurately.

Before presenting the noise radiation spectra due to the various aerofoil geometries under investigation, flat plate interaction noise measurements were made inorder to validate the measurement procedure and the flat plate theory due to Amiet (1975). 


\subsection{Flat plate validation}

The interaction noise generated by isotropic homogeneous turbulent flow of $2.5 \%$ turbulence intensity and $7.5 \mathrm{~mm}$ integral length scale, impinging on flat plates of thickness 1.5 $\mathrm{mm}$ was measured on the open jet wind tunnel facility at the ISVR. The flat plate of $0.45 \mathrm{~m}$ span and $0.15 \mathrm{~m}$ chord was located with the side plates of a rectangular nozzle of dimensions $0.45 \mathrm{~m} \times 0.15 \mathrm{~m}$ and the noise measured at a polar array of 11 microphones located $1.2 \mathrm{~m}$ from the aerofoil leading edge. Details of the measurement facility are presented in the Appendix A.

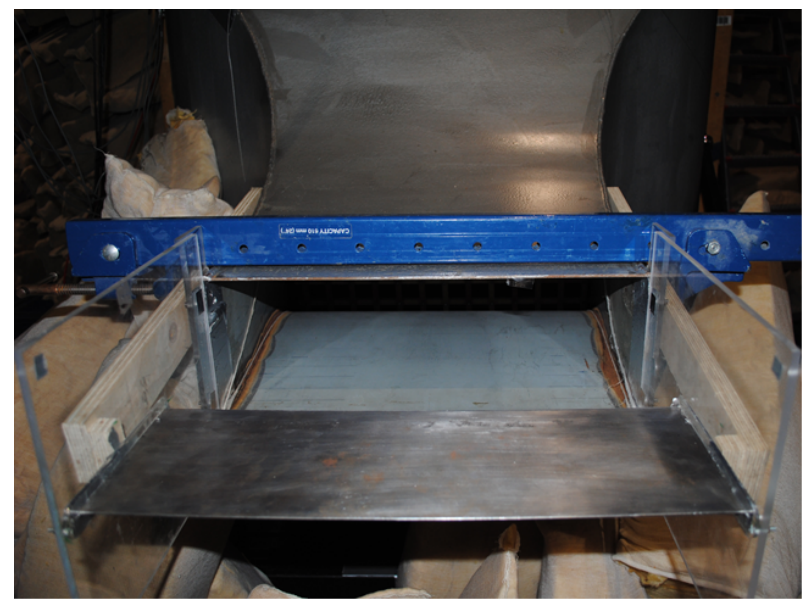

FIGURE 2.1: A photograph of flat plate test specimen mounted at the nozzle exit with the support of side plates.

In this preliminary study a flat plate of $1 \mathrm{~mm}$ thickness was introduced into the potential core of the open jet. Both the trailing and leading edges were sharpened in order to avoid vortex shedding as well as to provide a better approximation to a zero-thickness flat plate. A photograph of the flat plate model placed in the wind tunnel is shown in figure 2.1. The PSD of sound pressure radiated at $90^{\circ}$ to the jet axis are compared in Figure 2.2 against Amiet's flat plate analytic theory for different chord lengths of $75 \mathrm{~mm}, 100 \mathrm{~mm}, 150 \mathrm{~mm}$ and $200 \mathrm{~mm}$ respectively at $20 \mathrm{~m} / \mathrm{s}$ to $80 \mathrm{~m} / \mathrm{s}$. The description of Amiet's analytic theory is described in Appendix B. The radiated noise spectra exhibits the classical oscillations due to interference between the leading and trailing edges and are in excellent agreement with the flat plate theory for all cases at frequencies where interaction noise is dominant. Below this frequency the predictions are underestimated due to contamination of the measured spectra due to hydrodynamic pressure fluctuations from the turbulent jet shear layers.

The measured pressure directivity at the 11 microphone positions are compared in Figure 2.3 against Amiet's flat plate analytic theory for different chord lengths of $75 \mathrm{~mm}, 100 \mathrm{~mm}$, $150 \mathrm{~mm}$ and $200 \mathrm{~mm}$ at jet velocity of 20 to $80 \mathrm{~m} / \mathrm{s}$. These are plotted for different nondimensional frequencies $f c_{0} / a=0.5,1,1.5$ and 2 . At low frequencies the flat plate behaves like a compact dipole source and exhibits classical cardioid directivity. As non-dimensional frequency increases, Figure 2.3 reveals the presence of multiple side lobes in both measurement and prediction.

In the present sections of this chapter, far-field spectral plots are presented in terms of the Sound Pressure Level spectrum $S P L(f)$, defined in Eq. (2.1), and the Sound Power Level 


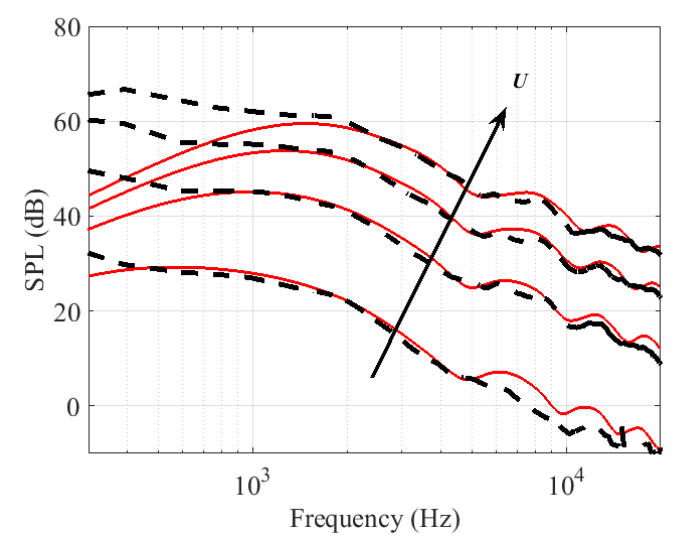

(a) $75 \mathrm{~mm}$ chord

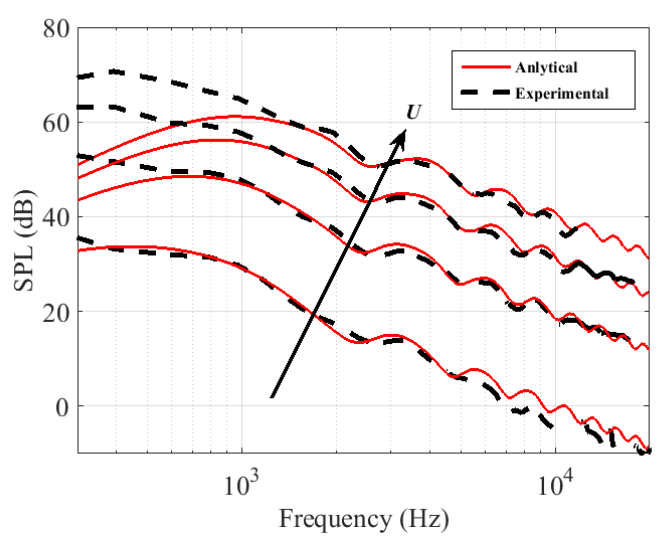

(c) $150 \mathrm{~mm}$ chord

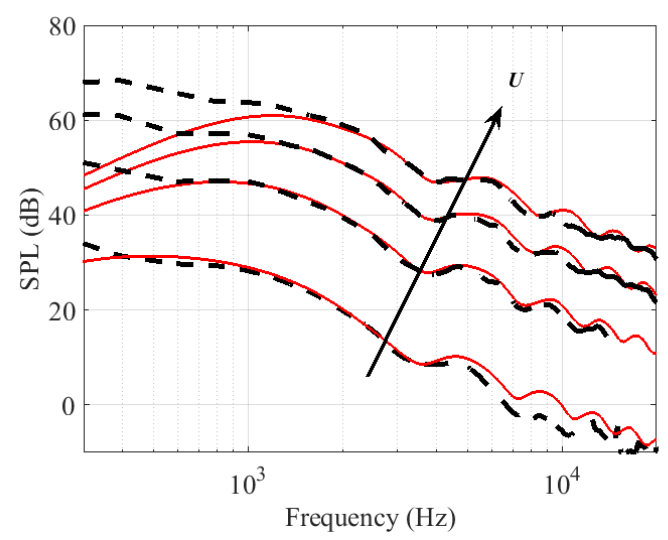

(b) $100 \mathrm{~mm}$ chord

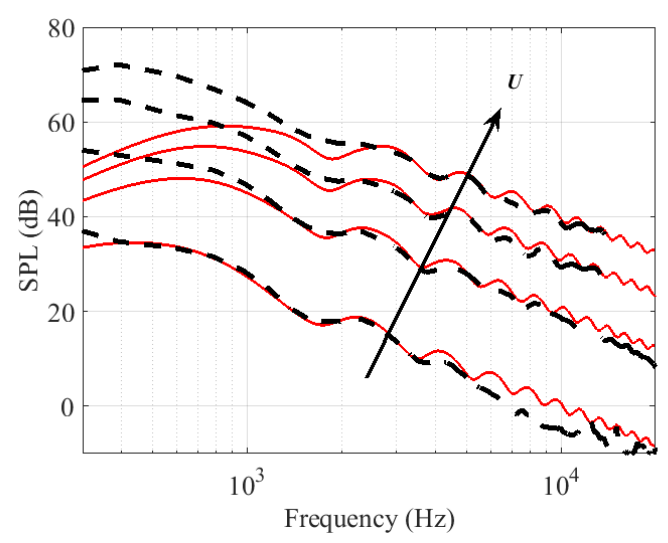

(d) $200 \mathrm{~mm}$ chord

FIGURE 2.2: Comparison of sound pressure levels at the $90^{\circ}$ microphone location between Amiet's analytic prediction (solid red) and experimental (dotted black) for varying chord ( $c_{0}=75 \mathrm{~mm}, 100 \mathrm{~mm}, 150 \mathrm{~mm}$ and $200 \mathrm{~mm}$ ) and varying jet velocities $(U=20,40,60$ and $80 \mathrm{~m} / \mathrm{s})$ 


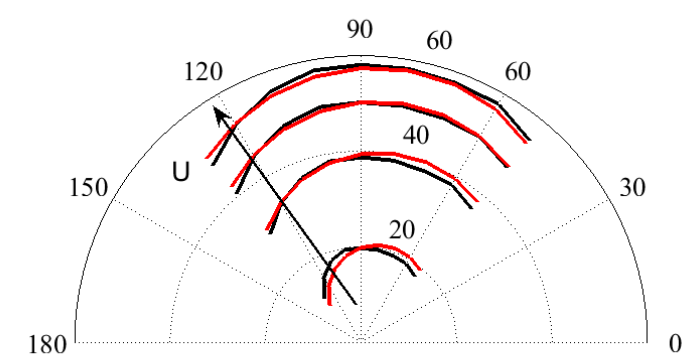

(a) $75 \mathrm{~mm}$ chord, $f c_{0} / a=0.5$

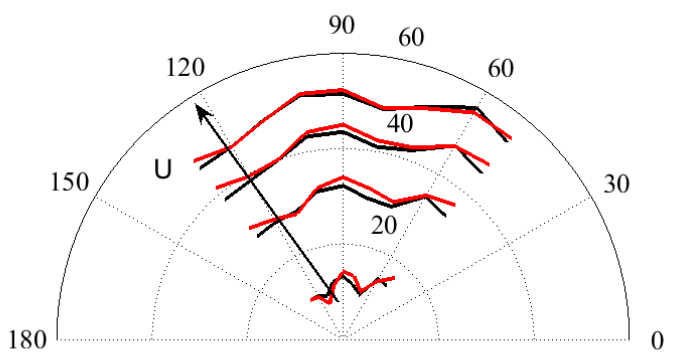

(c) $150 \mathrm{~mm}$ chord, $f c_{0} / a=1.5$

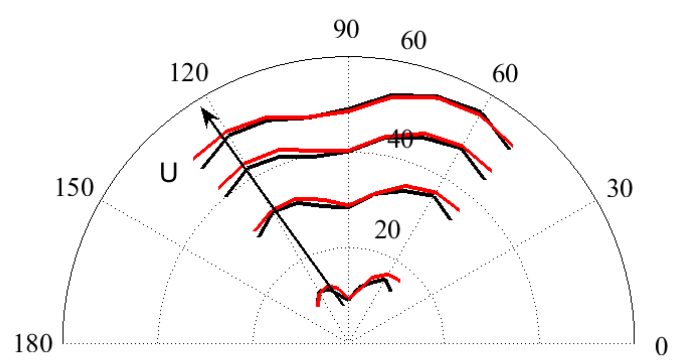

(b) $100 \mathrm{~mm}$ chord, $f c_{0} / a=1$

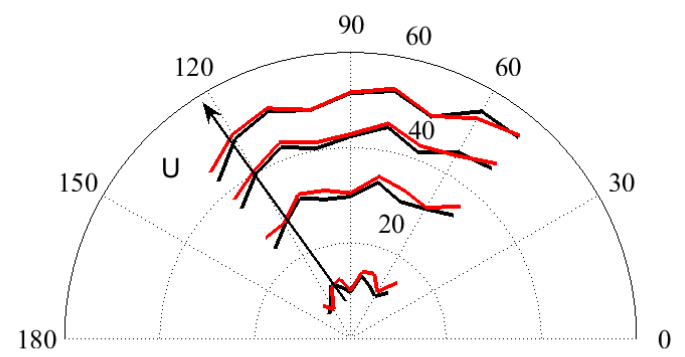

(d) $200 \mathrm{~mm}$ chord, $f c_{0} / a=2$

FIGURE 2.3: Comparison of directivity trends at different non-dimensional frequencies between Amiets analytic prediction (red) and experimental (black) for varying chord $\left(c_{0}=75 \mathrm{~mm}, 100 \mathrm{~mm}, 150 \mathrm{~mm}\right.$ and $\left.200 \mathrm{~mm}\right)$ and jet velocities $(U=20,40,60$ and $80 \mathrm{~m} / \mathrm{s})$ 
spectrum $P W L(f)$ defined in Eq. (2.2), estimated assuming cylindrical radiation,

$$
S P L(f)=10 \log _{10}\left(S_{p p}(f) / p_{r e f}^{2}\right)
$$

where $S_{p p}(f)$ is the power spectral density and $p_{\text {ref }}$ is $20 X 10^{-6} \mathrm{~Pa}$, and

$$
\begin{gathered}
W(f)=\frac{L R}{\rho a} \sum_{i=1}^{i=N}\left(\frac{S_{p p}\left(f, \theta_{i}\right)+S_{p p}\left(f, \theta_{i+1}\right)}{2}\right) \Delta \theta \\
P W L(f)=10 \log _{10}\left(W(f) / W_{\text {ref }}\right)
\end{gathered}
$$

where, $W(f)$ is the spectral density of sound power radiated between the radiation angles [ $40^{\circ}$ to $140^{\circ}$ ], $W_{\text {ref }}=10^{-12} \mathrm{~W}$ and $S_{p p}$ is the acoustic pressure PSD (Power Spectral Density) measured at measurement angle $\theta_{i}$ and $N$ is the number of microphones, $L$ is the span of the aerofoil [450 mm], $R$ is radius of the microphone array $(1.2 \mathrm{~m}), \Delta \theta$ is the angle between adjacent microphones (5-10 degrees), $\rho$ is the mass density of the ambient air, and $a$ is the speed of sound.

In the remaining sections of this chapter, the sound power level reductions $\Delta P W L(f)$ relative to the flat plate radiation plotted above defined as follows.

$$
\triangle P W L(f)=P W L_{0}(f)-P W L_{t}(f)
$$

where $P W L_{0}$ and $P W L_{t}$ are the radiated sound power for flat plate and aerofoil respectively are calculated based on Eq. 2.3. The chord and span for all the aerofoils are equal to the values for the flat plate ( $150 \mathrm{~mm}$ and $450 \mathrm{~mm}$ respectively).

\section{Effect of Chord}

Sound power level spectra are calculated using Equation 2.3 for flat plates of chord lengths of $75 \mathrm{~mm}, 100 \mathrm{~mm}, 150 \mathrm{~mm}$ and $200 \mathrm{~mm}$. These sound power spectra are plotted in figure 2.4 against frequency to observe the effect of chord length on turbulence-aerofoil interaction noise. This figure shows a strong dependence of noise on chord at lower frequencies $(f<$ $1000 \mathrm{~Hz}$ ) but almost no effect at higher frequencies.

To examine this phenomenon further the variation in sound power at three fixed frequencies of $f \approx 600,900$ and $1900 \mathrm{~Hz}$ and three velocities of $U=20,40$ and $60 \mathrm{~m} / \mathrm{s}$ are plotted in Figure 2.5 against non-dimensional hydrodynamic frequency $\left(\mu_{h}=\omega c_{0} / U / \beta^{2}\right)$. This figure reveals two regions of behaviour. One is where the radiated sound power varies as $c_{0}^{1}$ and the other which is proportional to $c_{0}^{0}$. The line that delineates these two regions of behaviour is non-dimensional acoustic frequency $\mu_{a}=\omega c_{0} / a / \beta^{2}=\pi / 4$. At this frequency the hydrodynamic wavelength $\left(\mu_{h}\right)$ is greater than a quarter of the chord and the acoustic wavelength $\left(\mu_{a}\right)$. This behaviour was first identified by Blandeau (2011). He showed analytically that a $c_{0}^{1}$ scaling occurs when $\mu_{a} \leq \pi / 4 \leq \mu_{h}$, i.e., there is a mismatch between the acoustic and hydrodynamic wavelengths. He also showed that a $c_{0}^{0}$ scaling occurs when both hydrodynamic and acoustic wavelength are both short such that $\mu_{h}>\mu_{a} \gg \pi / 4$. These behaviour are clearly evident in the measured data in Figure 2.5. Finally, he showed that a $c_{0}^{2}$ is observed when both wavelengths are long, i.e., $\mu_{a}<\mu_{h} \ll \pi / 4$. This behaviour is obscured in the measured data by jet noise and is therefore not seen in Figure 2.5. 


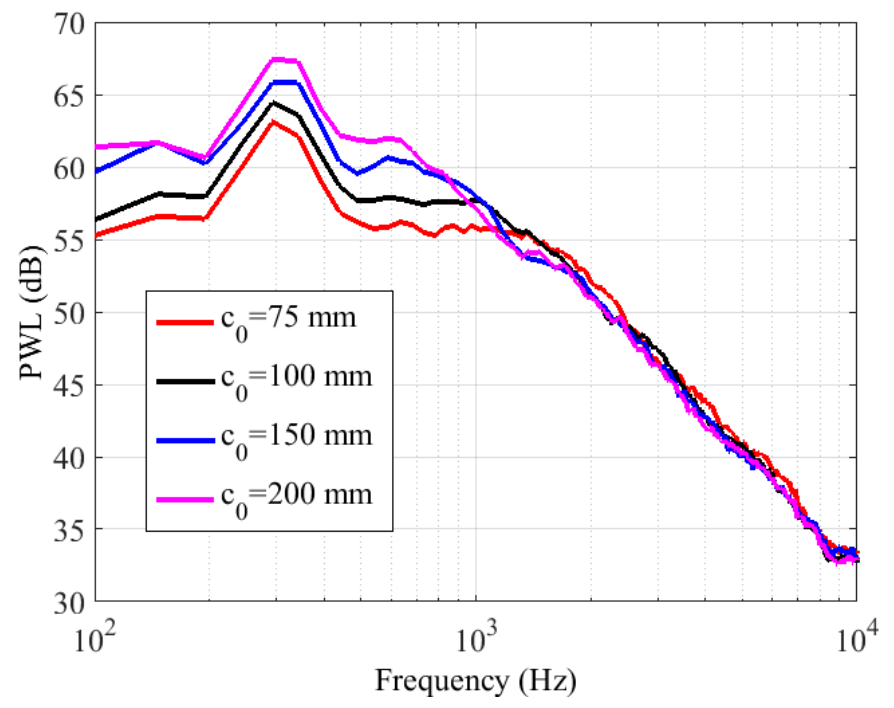

FIGURE 2.4: Power spectral comparison for different chord lengths varying from $75 \mathrm{~mm}$ to $200 \mathrm{~mm}$ at fixed jet velocity of $60 \mathrm{~m} / \mathrm{s}$.

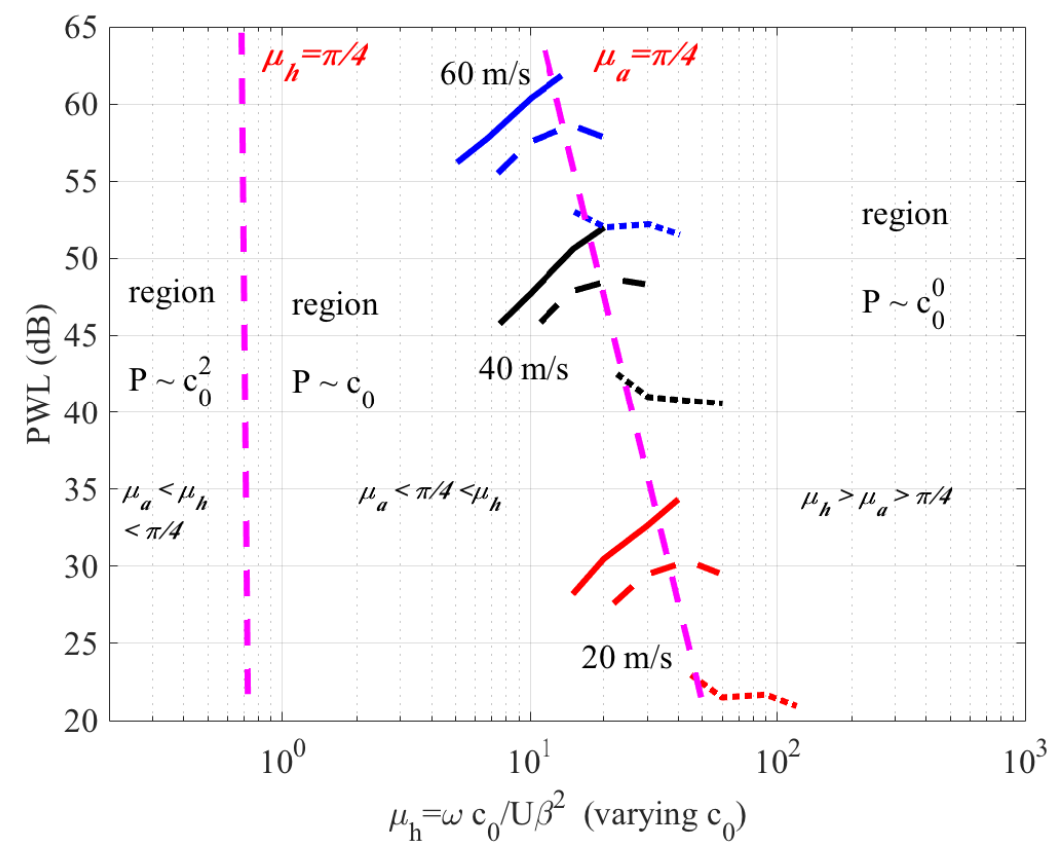

FIGURE 2.5: Effect of chord length on PWL for $U=20,40$ and $60 \mathrm{~m} / \mathrm{s}$, solid line - $f \approx 600 \mathrm{~Hz}$, dashed line $-f \approx 900 \mathrm{~Hz}$, dotted line $-f \approx 1900 \mathrm{~Hz}$

\subsection{Aerofoil Geometry effects}

\subsubsection{Aerofoil Configurations}

Following the flat plate theory validation against flat plate experimental data in Section 2.2, the current section focuses on the broadband noise radiation due to realistic aerofoil geometries. In this present study the NACA 4-digit modified series aerofoils were investigated. 


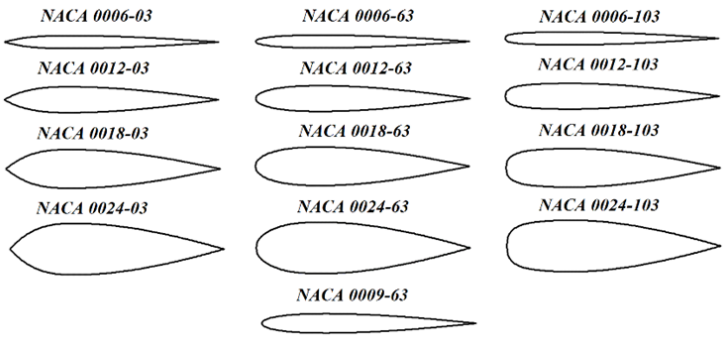

(a) Symmetric aerofoil geometries considered in the study

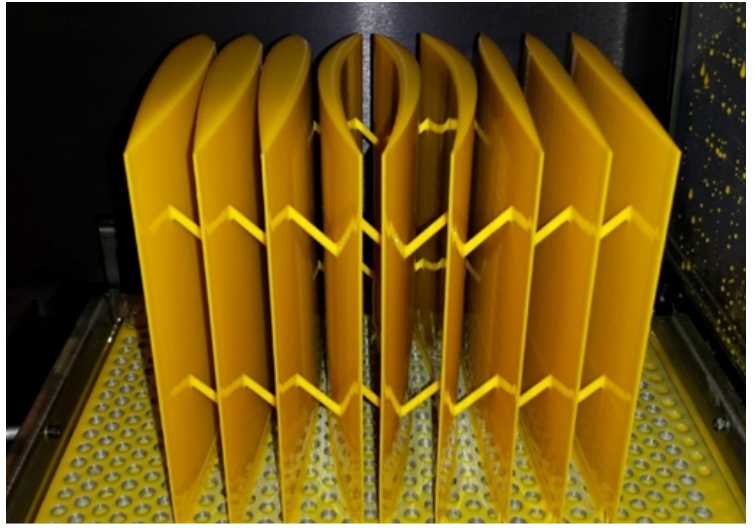

(b) Aerofoils in a 3D printer

FIGURE 2.6: Aerofoil Configurations.

Seventeen aerofoils were fabricated using 3D printing technology, of which 13 aerofoils are symmetric. The material used in the fabrication is envisionTEC's LS600, which are extremely durable photopolymers for use in producing accurate models with smooth surface finish. The aerofoils were chosen to have a systematically varying thickness and nose radius, as shown in Figure 2.6. In the case of the NACA 4-digit aerofoils, the leading edge nose radius $R_{e}$ is related to maximum thickness by

$$
R_{e}=0.5\left[0.2969 \frac{t}{0.2}\left(\frac{I}{6}\right)\right]^{2}
$$

where $\mathrm{I}$ is a non-dimensional parameter that defines the shape of the leading edge as shown in figure 2.6a (Gill et al. (2013)). I=0, represents a sharp leading edge, depicted as NACA xxxx-03, whereas I=10 represents blunt aerofoils, depicted as NACA xxxx-103 and I=6 represents standard NACA profile geometries.

The study has been performed on 3 leading edge nose radii ( $\mathrm{I}=0,6$ and 10), each of which has varying aerofoil thicknesses of $6 \%$ to $24 \%$ of chord as shown in Figure 2.6. The maximum thickness is positioned at $0.3 c_{0}$ from the leading edge. The dimensions of the chord $\left(c_{0}\right)$ and $\operatorname{span}(L)$ for all configurations are $150 \mathrm{~mm}$ and $450 \mathrm{~mm}$ respectively. The effect on interaction noise due to camber was also studied on four aerofoils of varying camber of $1 \%, 3 \%, 5 \%$ and $8 \%$ of chord (NACA 1406, 3406, 5406 and 8406). The position of maximum camber for all the aerofoils is $40 \%$ from the leading edge.

\subsubsection{Influence of Self-Noise}

In this section we show that the total noise radiated by the aerofoil located within a turbulent flow is dominated by leading noise at low frequencies and trailing edge noise at high frequencies. As it is very difficult to measure interaction and self-noise separately, our analysis was limited to the frequency region where the interaction noise dominates self-noise. Figure 2.7 shows the influence of self-noise on total noise spectra. Sound power levels are plotted against a non-dimensional frequency $(f t / U)$ for two different thickness aerofoils. It is observed that the thicker aerofoil has lower interaction noise and the self-noise starts dominating the total noise at lower frequency compared to the thinner aerofoil. The nondimensional frequency $(f t / U)$ where self-noise starts if the dominant noise source is around 


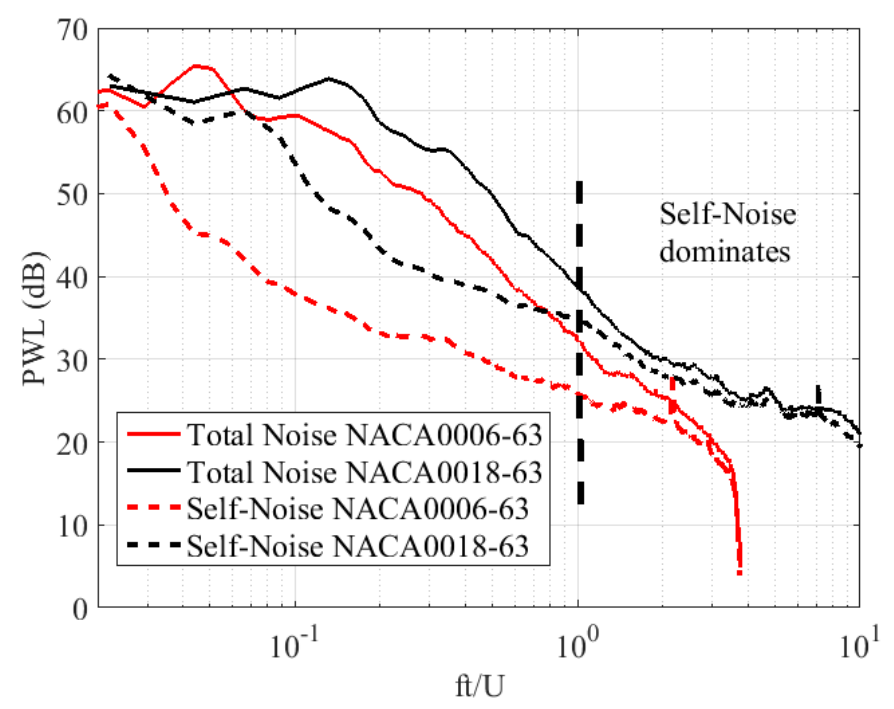

FIGURE 2.7: The Influence of Self-Noise on Total noise for $6 \%$ and $18 \%$ thickness to chord at jet velocity of $60 \mathrm{~m} / \mathrm{s}$.

1 for this particular turbulence intensity of about $2.5 \%$. For the case of flat plates, levels of self-noise were observed to be negligible over the frequency range of interest.

As aerofoil thickness was increased, trailing edge noise was found to increase in low frequency range and decrease in the high frequencies range as discussed by Chaitanya et al. (2015), due to a thickening of the boundary layer thickness. For these symmetric aerofoils Leung et al. (2016) has used the panel code XFOIL to demonstrate an almost linear variation of boundary layer thickness with aerofoil thickness. Thicker boundary layers near the trailing edge lead to larger scales of turbulence that contain more energy, and hence the increase in low frequency noise in the trailing edge noise spectra. The decrease in high frequency noise with thickness was attributed by Leung et al. (2016) to a decrease in the coefficient of friction. In this chapter, however, the analysis is restricted to the frequency range where interaction noise exceeds self noise by at least $5 \mathrm{~dB}$ and can therefore be neglected.

\subsubsection{Effect on interaction noise due to thickness}

The effect of aerofoil thickness on leading edge interaction noise is investigated in this section. The sound power level has been plotted against non-dimensional frequency $\left(f c_{0} / U\right)$ in Figure 2.8 for varying aerofoil thicknesses from $6 \%$ to $24 \%$ at fixed nose shape parameter $(I=6)$, for a jet velocity of $40 \mathrm{~m} / \mathrm{s}$. Consistent with earlier studies, Figure 2.8 clearly shows that the sound power level decreases with increasing aerofoil thickness.

As discussed in section 2.3.4, Gershfeld (2004) derived the simple expression $\exp (-\pi f t / U)$ to quantify the effect of aerofoil interaction noise relative to a flat plate by applying the trailing edge green's function to the leading edge of an aerofoil with thickness $t$. In this chapter we referred this correction as Howe's/Gershfeld's correction. This factor is now compared against measured interaction noise data.

Figures $2.9 \mathrm{a}, \mathrm{b}$ and $\mathrm{c}$ show the comparison of $-10 \log _{10}(\exp (-\pi f t / U))$ to the measured data for various thicknesses, non-dimensionalized by the maximum aerofoil thickness, $t$, for the three different nose shape factors, $I=0,6$ and 10. The comparison may be summarized as follows: 


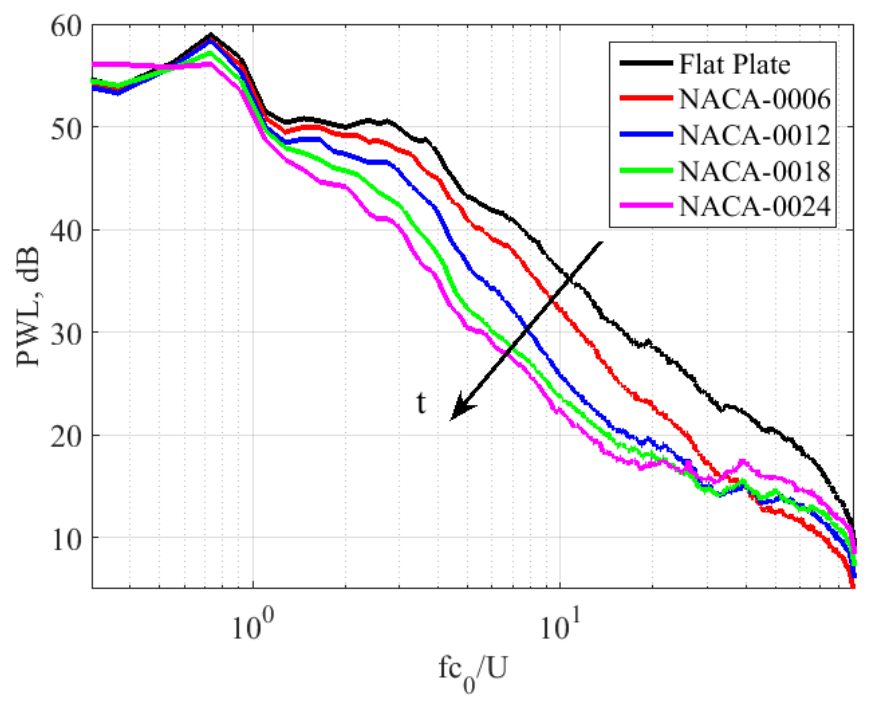

FIGURE 2.8: The effect on sound power spectra due to increasing thickness, with nose shape $\mathrm{I}=6$ and jet velocity of $40 \mathrm{~m} / \mathrm{s}$.

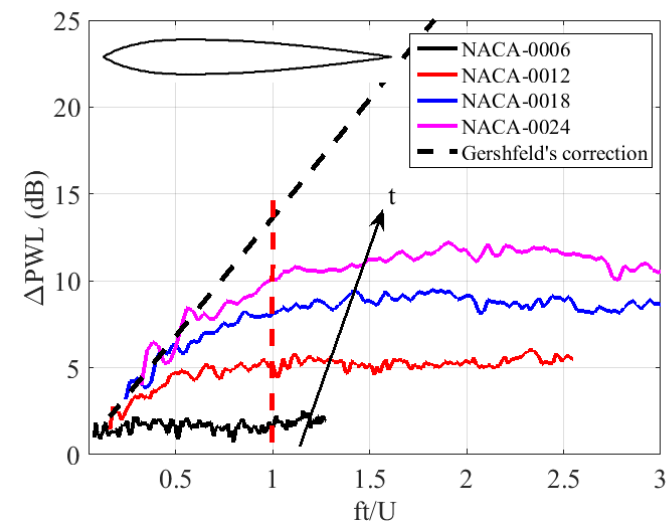

(a) $\mathrm{I}=0$

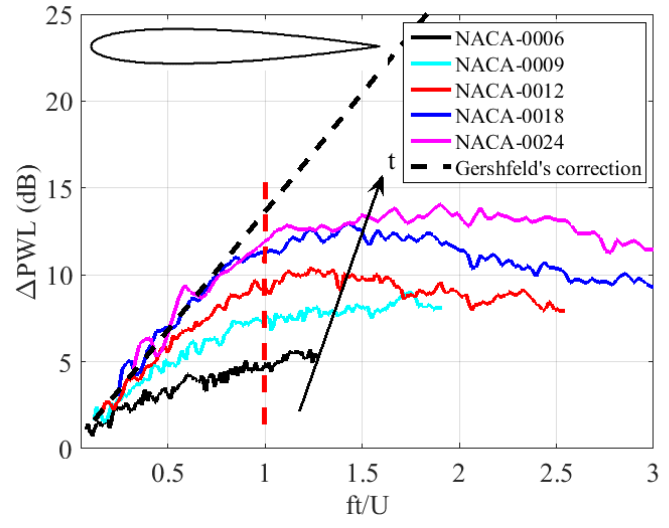

(b) $\mathrm{I}=6$

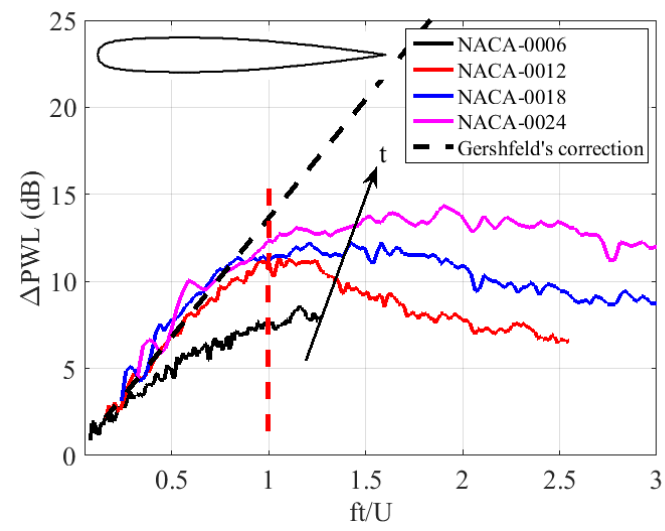

(c) $\mathrm{I}=10$

FIGURE 2.9: The effect on noise reductions due to increasing thickness with varying nose shape parameter 


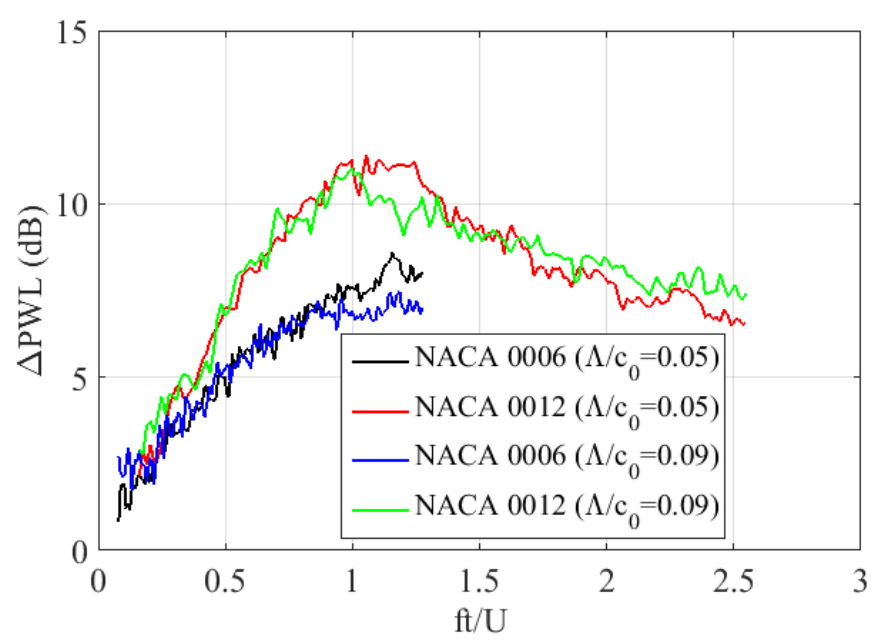

FIGURE 2.10: The Influence of integral length scale $(\Lambda)$ on Noise reductions for $6 \%$ and $12 \%$ thickness to chord $(I=6)$ at jet velocity of $60 \mathrm{~m} / \mathrm{s}$.

1. Except for the thinnest aerofoil, sound power reductions in Figure 2.9c are observed to follow the Howe prediction at low non-dimensional frequencies $f t / U$ but then deviate from it as frequency is increased. Thus, the correction factor appears to correspond to an upper limit sound power reduction.

2. As the aerofoil thickness is increased for the blunt aerofoils $I=10$ (Figure 2.9c), the correction factor is followed over a wider frequency range $f t / U$ before deviation occurs, although this may correspond to a smaller absolute frequency range.

3. For the aerofoils with the sharp leading edge $I=0$, Figure $2.9 \mathrm{a}$, the correction factor appears to deviate over a wider non-dimensional frequency range.

4. The reason for the departure from prediction is partly due to the actual interaction noise mechanism itself but is also partly due to the contribution from aerofoil selfnoise, which starts to dominate above some non-dimensional frequency $\mathrm{ft} / U$ roughly about 1 . Self-noise is particularly important for the thick, blunt aerofoils.

The influence of turbulence integral length scale $(\Lambda)$ on interaction noise is now investigated. Differences in the interaction noise power spectra relative to a flat plate were measured for the NACA0006 and NACA0012 aerofoils at the two integral length scales $\Lambda / c_{0}$ of 0.05 and 0.09 . These differences in sound power spectra are plotted in figure 2.10 against $f t / U$. It clearly shows that, even though turbulence length scale affects the sound power radiation, as shown explicitly by Amiet, integral length scale has a negligible effect on the difference in sound power radiation relative to a flat plate. It would appear that length scale affects interaction noise from thick aerofoils in precisely the same way as for flat plates, i.e, the sound power radiation is proportional to the correlation length which in turn can be related to the integral length scale.

\subsubsection{Comparison of thickness-based empirical models for the prediction on in- teraction from aerofoils}

In this section the various correction factors derived previously to predict the difference in the sound power radiated by thick aerofoils to a flat plate are now compared to the measured data. The radiated far field power spectral density given in Equation B.9 for a flat plate 
has been modified by an exponential correction factor to give the radiated far field power spectral density for an aerofoil of arbitrary thickness $t$. In this section, three correction factors are investigated, which are reformulated here to allow direct comparison between them. All three of three of these correction factors may be expressed as an exponential factor with non-dimensional factor $\beta(t)$, which is solely a function of aerofoil geometry, of the form

$$
\left.S_{p p}(X, Y, Z, \omega)\right|_{t}=\left.S_{p p}(X, Y, Z, \omega)\right|_{0} \exp \left[-\beta(t) \frac{f c_{0}}{U}\right]
$$

Hence the noise reductions on sound power level due to aerofoil thickness with reference to flat plates are calculated using:

$$
\triangle P W L=P W L_{0}-P W L_{t}=10 \log _{10}\left(\left.S_{p p}\right|_{0} /\left.S_{p p}\right|_{t}\right)
$$

where positive sign in noise reduction shows the reduction of turbulence-aerofoil interaction noise with the increase of aerofoil thickness. The measured noise reductions can only be expressed as per Eq. 2.6 for nose shapes $I=6$ and $I=10$. For the case of $I=0$, the noise reductions doesnt obey Eq. 2.6. Gershfeld's correction can be rewritten in terms of an attenuation coefficient as:

$$
\beta(t)=\pi\left(t / c_{0}\right)
$$

Lysak et al. (2013); Lysak (2011) developed an empirical expression for $\beta(t)$ based on timedomain calculations, accounting for the gust distortion by the mean flow around the aerofoil. In the study, Lysak considered NACA 65 aerofoils of different thickness to chord ratio in the range of $4 \%$ to $20 \%$. The leading edge radius in the case of the NACA 65 aerofoil will be lower than NACA symmetric aerofoils due to profile definition in which case the predictions are slightly underestimated.

By fitting the numerical predicted noise data Lysak et al. (2013) obtained second order polynomial fit is given by:

$$
\beta(t) \approx 0.75\left(t / c_{0}\right)+12\left(t / c_{0}\right)^{2}
$$

Moriarty et al. (2005) developed an empirical relation for the noise reduction using a boundary element method. His correction expressed as a $\beta$ factor is of the form:

$$
\beta(t)=\frac{2 \pi}{10 \log 10(e)}\left(1.123 D(t)+5.317 D(t)^{2}\right)
$$

where

$$
D(t)=\left(t / c_{0}\right)_{1 \%}+\left(t / c_{0}\right)_{10 \%}
$$

is termed the inflow turbulence noise indicator Moriarty et al. (2005).

The first two correction factors discussed above account for the effects of aerofoil thickness on interaction noise based only on the maximum thickness $t$. the third expression of Eq. 2.10 is based on two thickness at $1 \%$ and $10 \%$ chord. All three are compared against the noise data for the present aerofoil geometries and plotted against the measured attenuation coefficients. Figure 2.11 compares the variation in the attenuation coefficient $\beta$ with aerofoil maximum thickness for the three expressions. The measured data is classified into two cases. One is for the standard NACA profiles $(I=6)$. The second is for the blunt NACA profile 


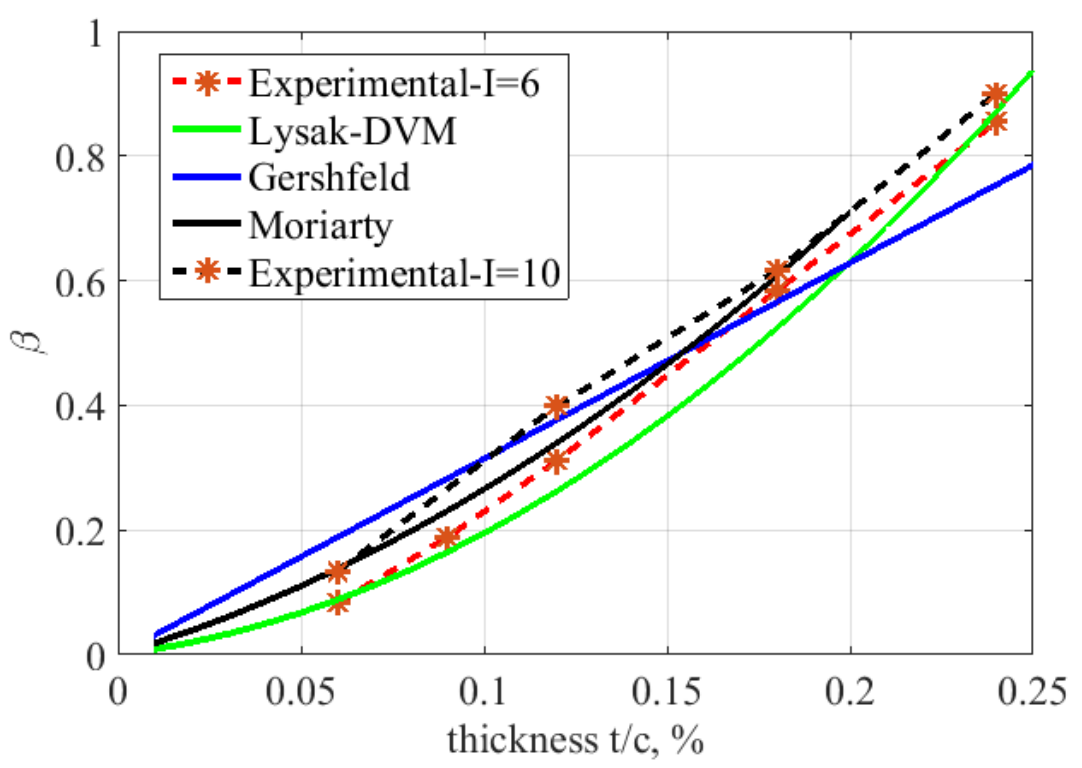

FIGURE 2.11: Attenuation coefficient as a function of aerofoil relative thickness plotted against models available in literature

$(I=10)$. Clear differences in the variation between the two curve fits is apparent due to the increase in nose radius. A second order polynomial expression in $t / c_{0}$ was fitted to the measured data for both aerofoils $I=6$ and 10, as follow:

$$
\begin{aligned}
& \beta(t)=1.369\left(t / c_{0}\right)+9.429\left(t / c_{0}\right)^{2} ; \text { for } I=6 \\
& \beta(t)=2.448\left(t / c_{0}\right)+5.493\left(t / c_{0}\right)^{2} ; \text { for } I=10
\end{aligned}
$$

For the case of a sharp nose radius, the attenuation coefficient is a function of the frequency and hence it is not plotted in the Figure 2.11.

A unified model has been attempted to account for the two different nose shapes and the fit is shown in Figure 2.12. In Figure 2.12 the attenuation coefficient is plotted against the inflow turbulence noise indicator and a reasonable fit has been established for two different nose shapes, which is given by

$$
\beta(t)=1.911 D(t)+3.856 D(t)^{2}
$$

The comparison between the expressions for $\beta$ is summarized as follows:

1. Considering only the maximum thickness in the attenuation coefficient does not capture the physics of interaction noise generation completely. The nose shape has been shown to also have an effect from figure 2.11.

2. Lysak's model predicts the interaction noise spectrum, even for thinner aerofoils even though it was derived on the noise data from NACA 65 aerofoils. The Lysak's model is based on predictions using rapid distortion theory to properly account for the aerofoil geometry on interaction noise. Thus for the geometries considered, Lysak's model predictions are reasonable for regular nose shape parameter $I=6$. 
3. Gershfeld's model is a simpler model that does not consider the behaviour of mean flow in the vicinity of the leading edge profile which naturally leads to discrepancies with experimental noise data.

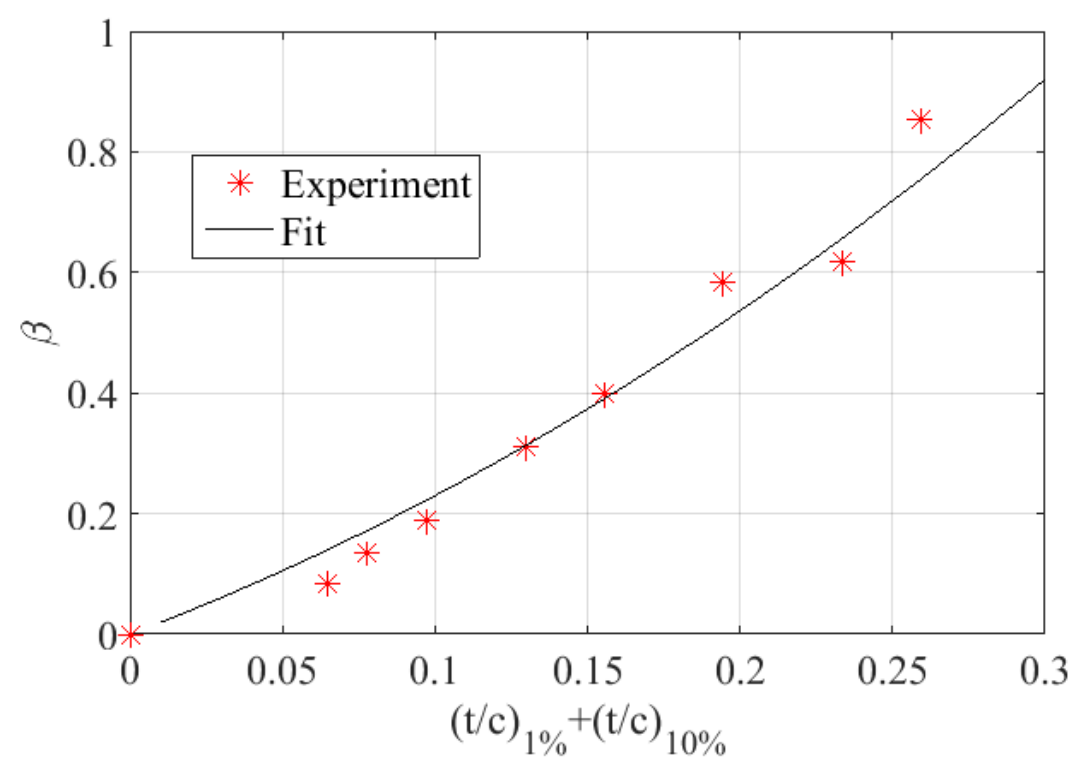

FIGURE 2.12: Attenuation coefficient as a function of inflow turbulence noise indicator

\subsubsection{Effect on interaction noise due to leading edge nose radius}

The present section investigates the effect of leading edge nose radius on interaction noise. The sound power level is plotted against non-dimensional frequency $\left(f c_{0} / U\right)$ in Figure 2.13 for varying nose shape parameters of $\mathrm{I}=0,6$ and 10 for the $6 \%$ thickness aerofoil for a jet velocity of $40 \mathrm{~m} / \mathrm{s}$. The sound power level is observed to decrease with increasing nose radius above the frequency at which jet noise can be neglected. As in Figure 2.9, the noise reduction versus frequency increases steadily until a certain frequency at which self-noise starts to become significant.

Noise reductions plotted against non-dimensional frequency $(f t / U)$ for increasing nose radius at two different aerofoil thickness of $6 \%$ and $12 \%$ are plotted in Figure 2.14. The effect of nose radius is seen to be more pronounced for thinner aerofoils than for thicker aerofoils, whose sensitivity to nose radius is very weak. This again shows that the thickness correction factor derived by Gershfeld/Howe represents the upper limiting value.

Figure 2.15 is a plot of the sound power level reduction at different jet velocities plotted against $f t / U$. Excellent collapse of the difference in sound power spectra relative to a flat plate suggesting that the important factor in determining the power radiation is the gust wavelength $U / f$ compared to some length-scale $L$ of the aerofoil, which is close to the maximum aerofoil thickness for thick aerofoil but is presently unclear for thinner aerofoils. 


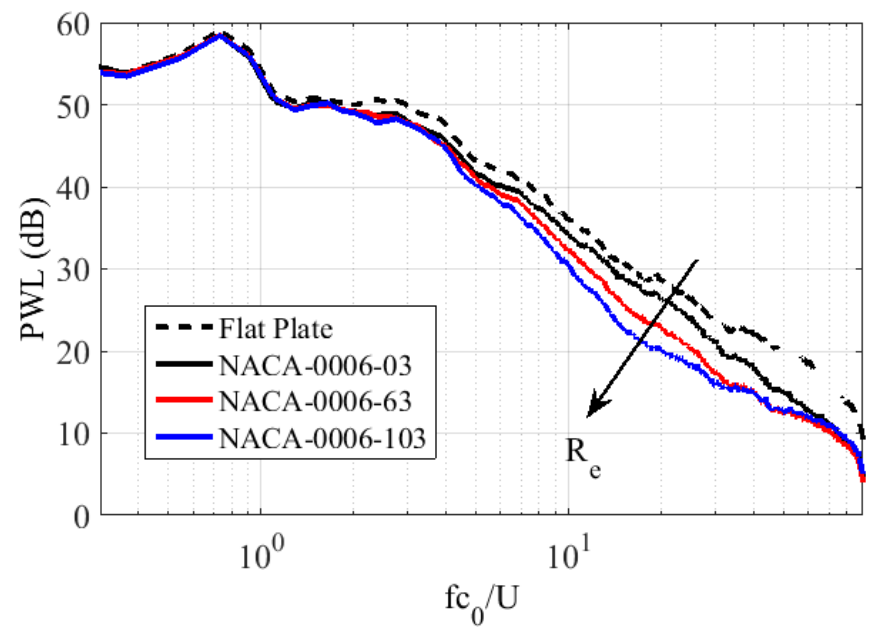

FIGURE 2.13: The effect on sound power spectra due to increasing nose radius, for a fixed thickness of $6 \%$ and jet velocity of $40 \mathrm{~m} / \mathrm{s}$.

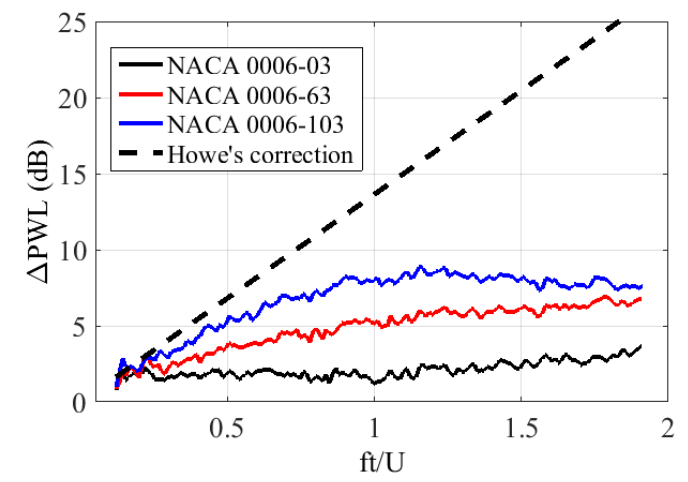

(a) thickness, $t / c_{0}=0.06$

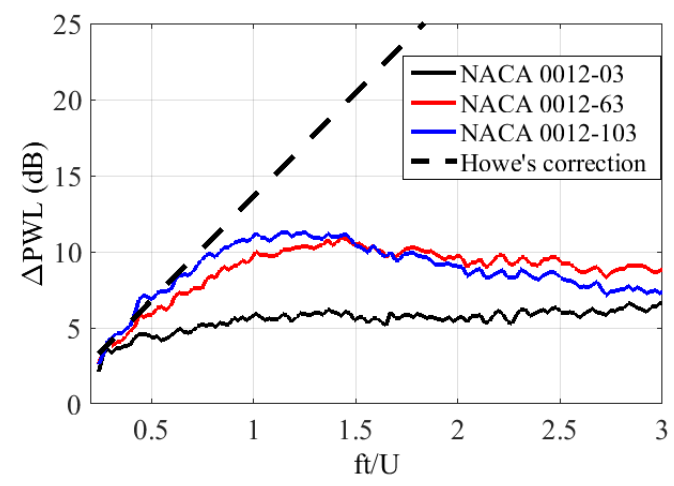

(b) thickness, $t / c_{0}=0.12$

FIGURE 2.14: The effect on noise reductions due to increasing nose radius for varying thickness.

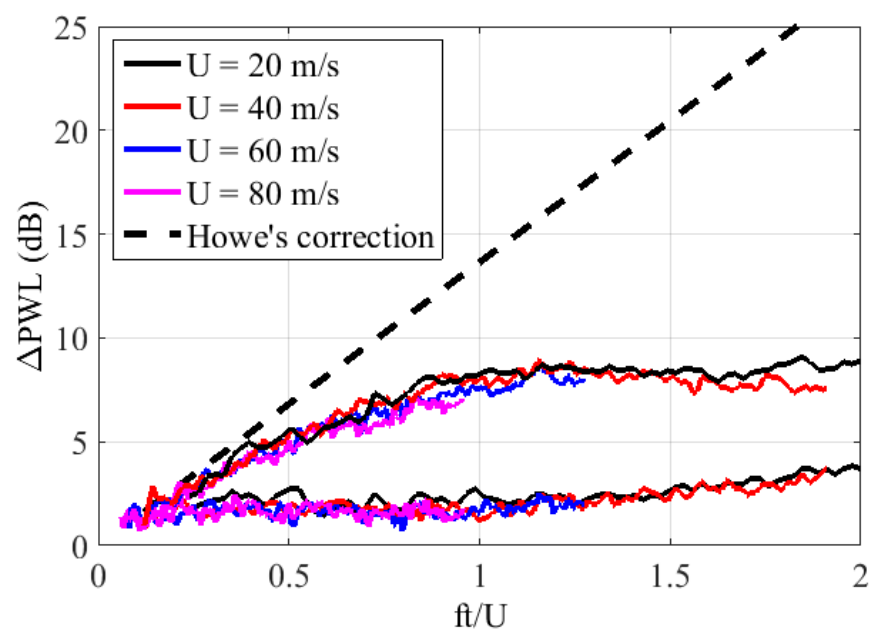

FIGURE 2.15: The effect on noise reductions due to varying jet velocities at two different nose shape parameters, $\mathrm{I}=0$ and $\mathrm{I}=10$ at a fixed thickness of $6 \%$. 


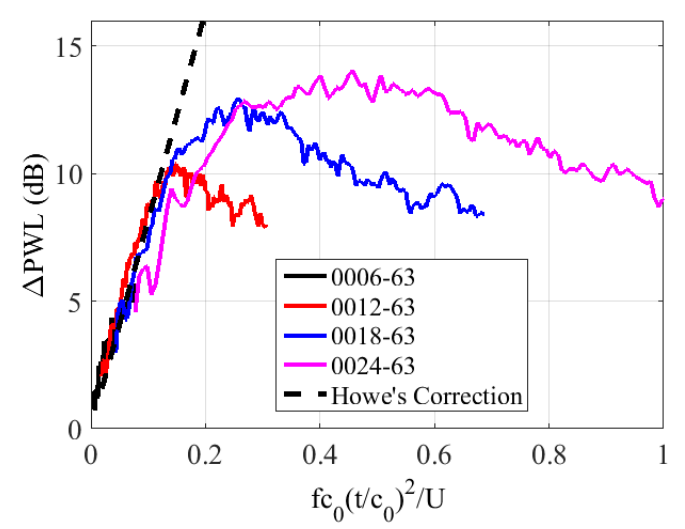

(a) $\mathrm{I}=6$

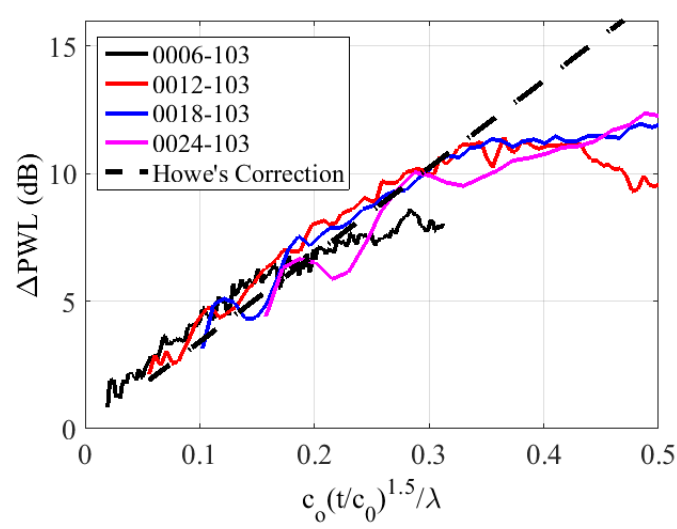

(b) $\mathrm{I}=10$

FIGURE 2.16: Noise reduction trends with increasing thickness for nose shape parameter, $\mathrm{I}=0,6$ and 10

\subsubsection{Discussion on thickness and nose radius effects}

Aerofoil thickness remains the main geometric parameter controlling turbulence interaction noise. Local leading edge geometry, such as leading nose radius, nevertheless, is an important parameter for thin aerofoils at high frequencies, particularly when the gust wavelength is comparable to nose radius. Therefore attempts to predict interaction noise based on single values of thickness-chord ratio are likely to be inaccurate for thin aerofoils.

Many attempts have been made to obtain a unified scaling factor relative to hydrodynamic gust wavelength $U / f$ governing sound power reductions. Figure 2.16a is a plot of the difference in sound power spectra relative to a flat plate for different aerofoil thicknesses at a constant nose radius of $I=6$. Reasonable collapse of the noise reduction spectra are observed for different aerofoil thicknesses when plotted against the non-dimensional frequency $\left(c_{o}\left(t / c_{0}\right)^{2} / \lambda\right)$ for nose shape parameter, $\mathrm{I}=6$. Thus, the difference in sound power spectra relative to a flat plate for standard NACA profiles are therefore mostly determined by the nose radius.

In the case of blunt aerofoils, ( $\mathrm{I}=10)$ noise reductions collapses on non-dimensional frequency $c_{o}\left(t / c_{0}\right)^{1.5} / \lambda$ as shown in Figure 2.16b. This difference in power law from 2 to 1.5 is mainly due to the difference in behaviors for the thinner aerofoils which exhibit a stronger influence on nose radius.

The scaling laws of the difference in sound power with $\left(t / c_{0}\right)$ above are not universal across different nose shapes and thicknesses. This again suggests that the details of aerofoil profile at positions upstream of the position of maximum thickness plays a key role in governing the sound power radiation.

\subsubsection{Effect of camber on turbulence-aerofoil interaction noise}

To study the effect of camber on turbulence-interaction noise, four different aerofoils of varying camber from $1 \%$ to $8 \%$ relative to chord are compared. camber is defined as the asymmetry between the two acting surfaces of an aerofoil. In this study the location of maximum camber is kept constant at $40 \%$ relative to chord. Figure 2.17 shows the sound power comparison between different aerofoils of different camber \% relative to the chord at a jet 


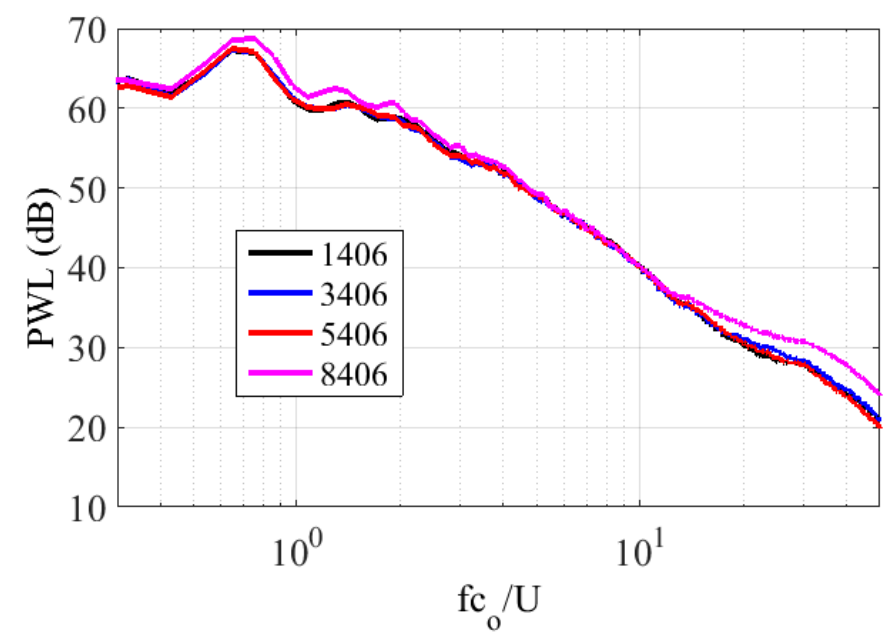

FIGURE 2.17: Effect of camber on turbulence-interaction noise.

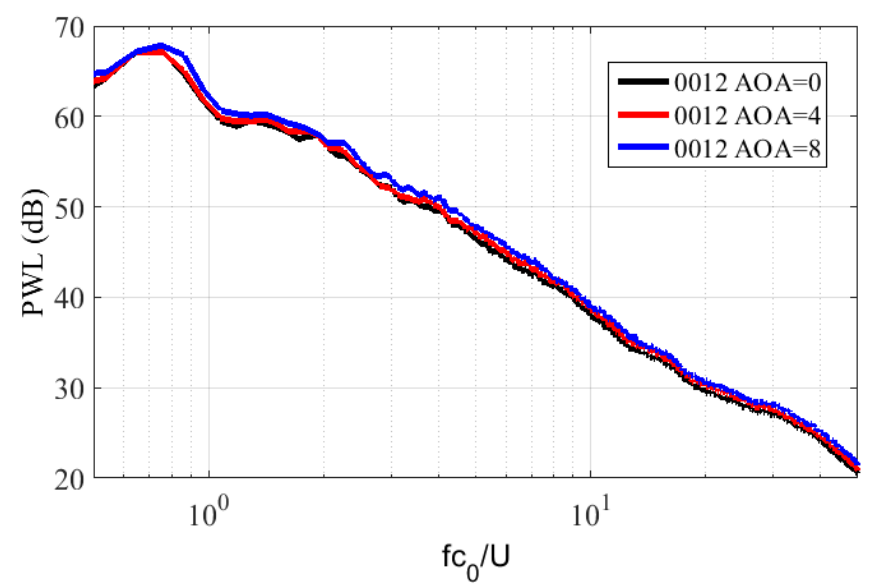

FIGURE 2.18: The effect on turbulence-interaction noise due to AOA.

velocity $U=68 \mathrm{~m} / \mathrm{s}$. The effects of camber are clearly negligible on turbulence-aerofoil interaction noise as previously observed by Devenport et al. (2010). Thus the parametric study of aerofoil thickness and nose radius on turbulence-aerofoil interaction noise is performed on symmetric NACA aerofoils as seen is section 2.3.3.

\subsubsection{Effect of AOA on turbulence-aerofoil interaction noise}

To study the influence of angle of attack on turbulence-aerofoil interaction noise, the noise measurements are performed at three different geometric angles of attack of $0^{\circ}, 4^{\circ}$ and $8^{\circ}$. Figure 2.18 shows the total sound power variation for NACA0012 aerofoil at three different geometric angles of attack $\left(\mathrm{AOA}=0^{\circ}, 4^{\circ}\right.$ and $\left.8^{\circ}\right)$. It is evident from the Figure 2.18 that the effect of AOA on turbulence-aerofoil interaction noise is negligible. Again, these results are consistent with the findings of Devenport et al. (2010), where the angle of attack effect is weak when the incoming flow is isotropic turbulence. 


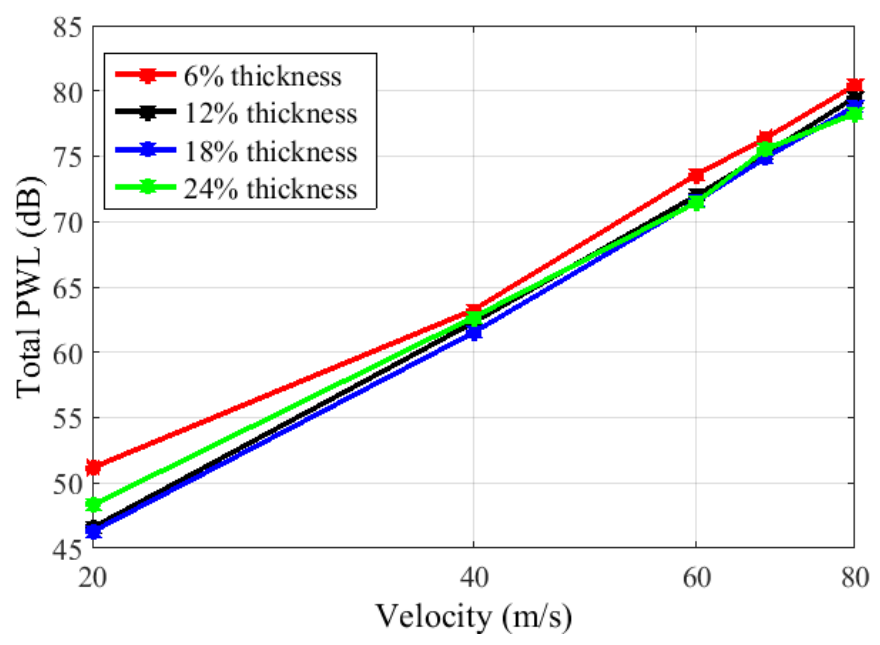

FIGURE 2.19: Total power versus velocity for varying thickness aerofoils from $6 \%$ to $24 \%$.

\subsection{Effects on interaction noise due to flow speed}

We now investigate the influence of flow speed on turbulence-aerofoil interaction noise. This analysis is useful to draw important conclusion to extrapolate our analysis to realistic mean flow and desirable frequency of interest.

The total sound power level is calculated by logarithmically adding the entire frequency range from $800 \mathrm{~Hz}$ to $10,000 \mathrm{~Hz}$. Total power radiated is plotted against velocity for aerofoils of varying thickness $6 \%$ to $24 \%$ in Figure 2.19 . The total noise follows a velocity scaling law of 5 to 6 , which is similar to a dipole type of source. As interaction noise is a dipole type behaviour we observe a similar velocity scaling $\left(\approx U^{5.5}\right)$ with total sound power.

Sound power reductions versus $f t / U$ are shown in Figure 2.20 at varying jet velocities of $20,40,60$ and $80 \mathrm{~m} / \mathrm{s}$ for two different aerofoil thicknesses of $6 \%$ and $12 \%$ and for a fixed nose shape parameter, $\mathrm{I}=6$. Noise reduction spectra are observe to collapse extremely well when plotted against $f t / U$. It clearly shows that, even though jet velocity affects the sound power radiation, as shown explicitly by Amiet, jet velocity has a negligible effect on the difference in sound power radiation relative to a flat plate. It would appear that jet velocity affects interaction noise from thick aerofoils in precisely the same way as for flat plates, i.e, the sound power radiation is proportional to $U^{5.5}$. Hence noise reductions follow a Strouhal dependence $\Delta P W L(f L / U)$, where $L$ is some appropriate length-scale based on the thickness and nose shape parameters.

A similar analysis has been extended to high jet velocities using CAA. Details of the CAA analysis used in the current chapter is described in Chaitanya et al. (2015). Sound power reductions at velocities of 60 to $200 \mathrm{~m} / \mathrm{s}$ are shown in Figure 2.21 versus $\mathrm{ft} / \mathrm{U}$ for a fixed nose radius of $I=6$ and thickness $12 \%$ relative to chord. The noise reductions are calculated using Amiet's analytical model. The change in power level characteristics at low and high speeds is almost similar, especially below the non-dimensional frequency 2 . The effects at different Mach numbers were observed from the figure 2.21, and the data gives a good collapse up to $M=0.587$.

All measured and predicted noise data exhibit a dependence on velocity that varies as $U / f$. 


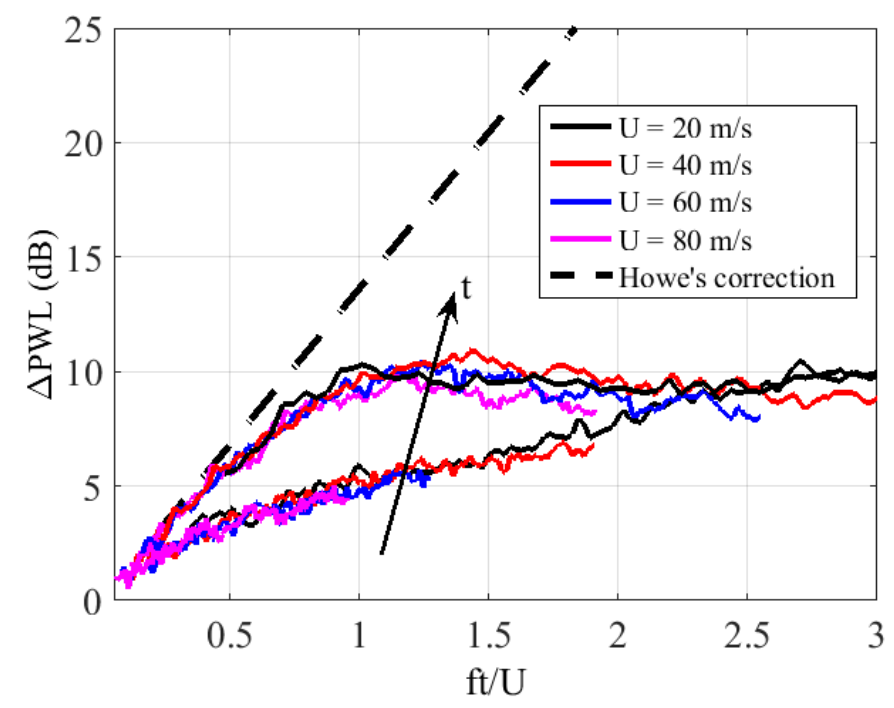

FIGURE 2.20: The effect on noise reductions due to varying jet velocities at two thickness, $t=6 \%$ and $t=12 \%$ at a fixed nose shape, $\mathrm{I}=6$.

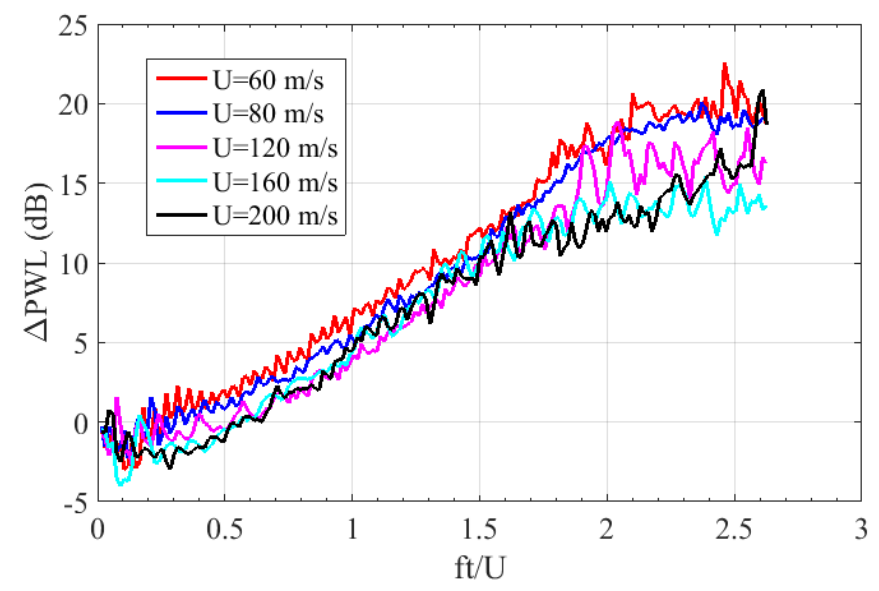

FIGURE 2.21: The effect on noise reductions due to varying jet velocities using CAA, $t / c_{0}=12 \%$ at a fixed nose shape, $\mathrm{I}=6$.

Identical aerofoils for the same $\mathrm{U} / \mathrm{f}$ therefore have the similar change in sound power compared to a flat plate. The character of the radiated noise changes for aerofoils relative to flat plate were predicted to be identical at both low and high velocities, suggesting identical noise generation mechanism over the entire velocity range. Compressibility effects associated with high flow speeds are thus not predicted to introduce additional noise sources. Interaction noise measurements made in low speed wind tunnel therefore provide a valid assessment of noise generation that may be extrapolated to higher flow speeds.

As shown in the previous sections, the compressibility effects associated with high speed flow don't introduce additional noise sources. Thus, extrapolating the noise reductions frequency limit to realistic mean flow at approach conditions, the influence of nose radius is seen at $20 \mathrm{KHz}$ (Where $3 \mathrm{KHz}$ is the $3 \mathrm{BPF}$ at approach condition). This suggests small geometric changes such as thickness and nose radius don't effect the turbulence-aerofoil noise at realistic Mach numbers $(M=0.5-0.7)$, where broadband noise is dominant (until 3BPF) 


\subsection{Comparisons with CAA Simulations}

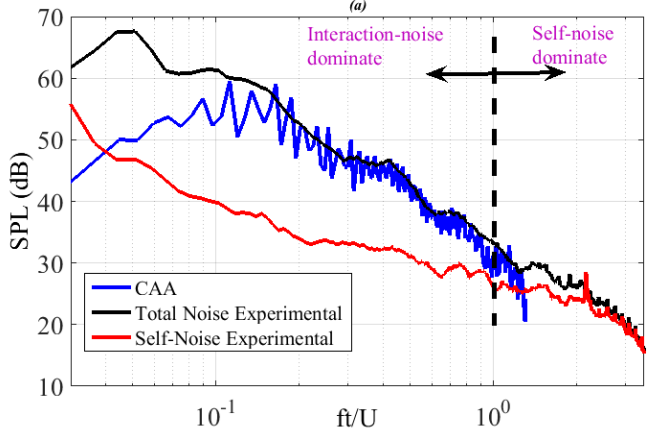

(a) NACA 0006-63

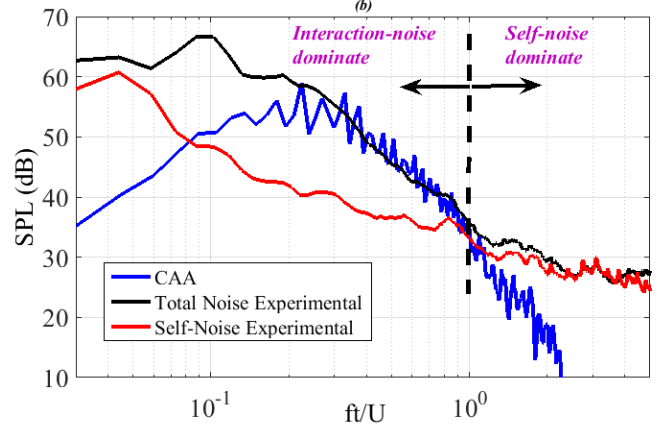

(b) NACA 0012-103

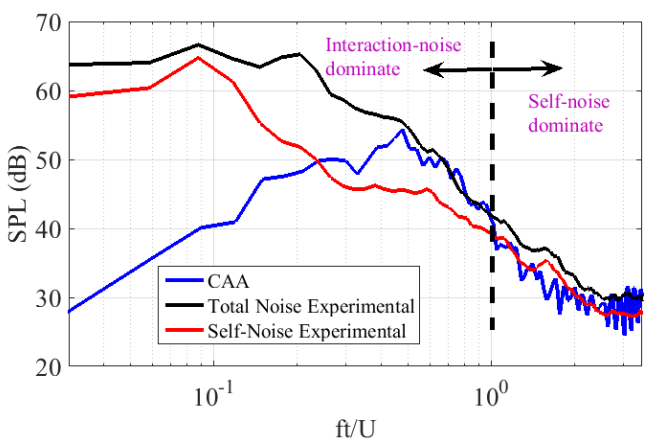

(c) NACA 0024-63

FIGURE 2.22: Sound pressure level comparison between CAA predictions and experimental data.

The experimental noise measurements are compared in Figure 2.22a, b and c with noise predictions from CAA simulations. CAA simulations are performed by ANTC group at the University of Southampton. The comparison is shown for three aerofoil geometries at an observer angle of $90^{\circ}$ in $M=0.176$ flow. In this comparison, $c_{0}=0.15 \mathrm{~m}, I=2.5 \%$, and $\lambda=7.5$ $\mathrm{mm}$. Close agreement of better than $2 \mathrm{~dB}$ is seen between the CAA predictions and the experimental measurements of turbulence-aerofoil interaction noise, for non-dimensional frequencies above 0.2 , where jet noise can be neglected from the experimental measurements. It is noted that the error in the experimental velocity might be around $3-5 \%$. This error doesn't change the noise reductions trends, which are shown in above section.

Also shown in this Figure (red curve), is the aerofoil self-noise, obtained by measuring the radiated noise in the absence of the turbulence grid. For the NACA 0012-103 aerofoil measurements, the aerofoil self-noise dominates at $f t / U>1$, which explains the discrepancy between prediction and experiment in this region. The computational method does not include self-noise and therefore predicts a lower noise amplitude. For the thicker aerofoils, the self-noise starts to dominate the total noise at lower frequencies. However, the threshold non-dimensional frequency, above which the trailing edge noise will dominate, is approximately around 1 for all three cases for a fixed turbulence generating grid. Figure 2.22 shows that the CAA simulations are capable of accurately predicting the turbulence interaction noise from thick symmetric aerofoils. 
It should be noted that the CAA simulation of the NACA 0024-63 aerofoil used an altered method that is described in this section. It has been previously found that there is a threshold limit of aerofoil thickness and frequency, above which it is no longer valid to neglect transverse Fourier components Gill (2015). Therefore, the NACA 0024-63 case required a more complete 3D turbulence synthesis method, which includes both streamwise and transverse vortical Fourier components. Additional detail on the turbulence synthesis method, given in reference Gill (2015), is not included here for brevity.

The sound power level predicted by the CAA method is calculated and compared against experimental measurements and Amiet's analytical solution. Figure 2.23 shows the sound power spectral comparison between the Experiment, the CAA, and Amiet's analytical flat plate model, at a jet velocity of $60 \mathrm{~m} / \mathrm{s}$. Close agreement is observed between all three approaches, particularly in the non-dimensional frequency range of 0.2 to 1 , where interaction noise is the dominant source mechanism compared to jet and self-noise in the experimental data. Amiet's flat plate theory is used to calculate the change in sound power level from CAA results at different jet velocities to address the compressibility effects.

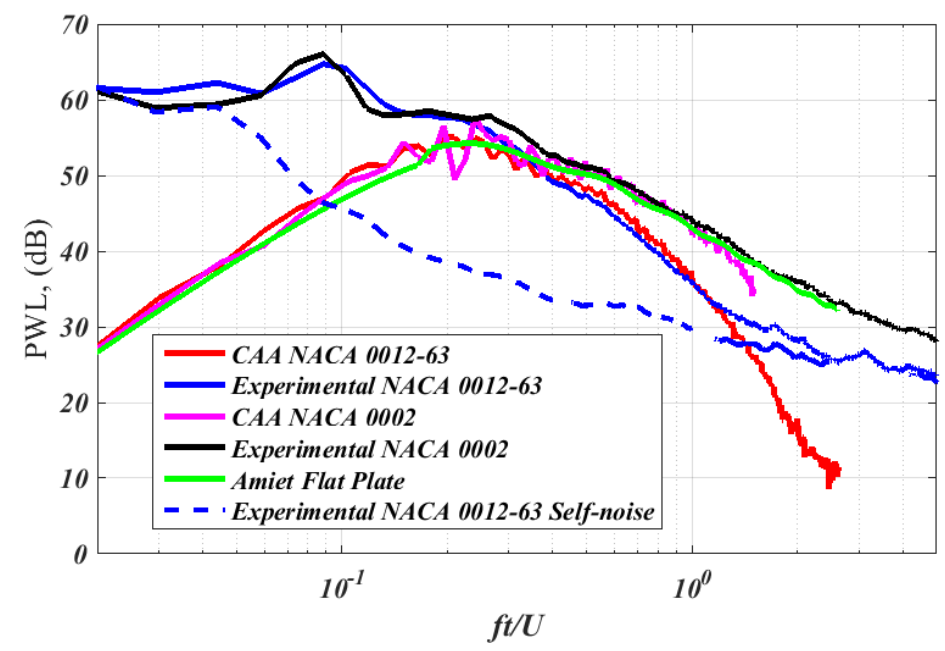

FIGURE 2.23: Sound Power spectral comparison between Experimental, analytical and CAA for aerofoil of thickness $12 \%$ of chord $\left(c_{0}\right)$ at jet velocity 60 $\mathrm{m} / \mathrm{s}$.

Figure 2.24 shows the comparison of the change in the sound power level, between experimental measurement and CAA predictions, at different jet velocities. We observe very good agreement of experimental change in sound power across all frequencies, for various jet velocities. We note that the measurements and predictions were performed completely independently by the different people independently and then compared. At low Mach numbers $(M \leq 0.35)$, the collapse of CAA predictions is reasonable at all frequencies. The deviation from the experimental noise reduction curves at low frequencies $(f t / U<=1)$ is because the flat plate analytic spectra are used in calculating CAA noise reductions. We also observe the change in sound power levels, due to thickness, starts at lower non-dimensional frequency in case of experiments than compared to CAA predictions. 


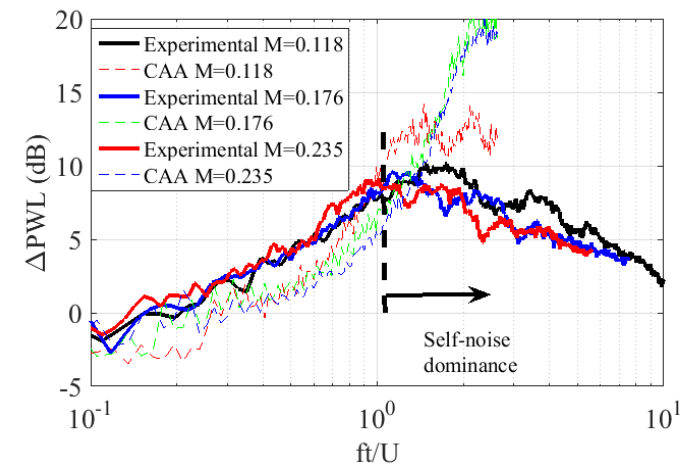

(a) $M \leq 0.35$

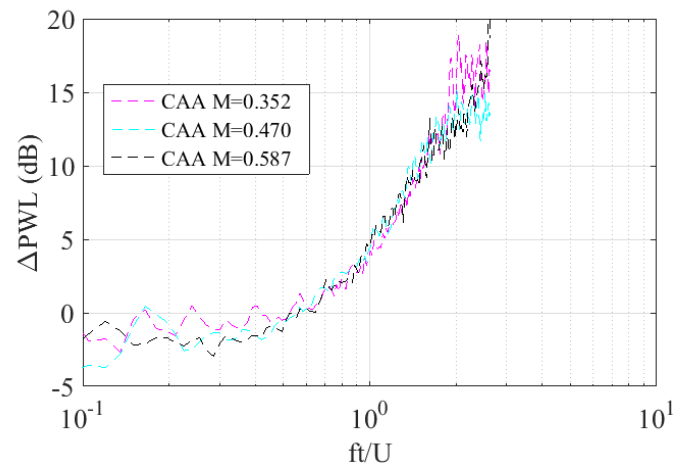

(b) $M \geq 0.35$

FIGURE 2.24: Sound power reductions for aerofoil of thickness $12 \%$ of chord $\left(c_{0}\right)$ at jet velocities 40 to $200 \mathrm{~m} / \mathrm{s}(M=0.118$ to 0.587$)$.

\subsection{Flow measurement}

Preliminary PIV results are presented to visualize the behaviour of the flow in the vicinity of the aerofoil edge. This was done with the purpose of understanding the leading edge noise interaction mechanism and the effect of thickness and nose radius. Figures 2.25a and b show the mean velocity streamlines around the NACA 0006-63 and NACA 0012-63 aerofoils. In the case of the thinner aerofoil, the stagnation region is smaller than that of the thicker aerofoil, in which the velocity gradients along the mean flow are much greater than the case of the smaller stagnation region. A small stagnation region results in high velocity fluctuations at the nose of the aerofoil, resulting in higher interaction noise.

Figures $2.25 \mathrm{c}$ and $\mathrm{d}$ show the mean velocity streamlines around the NACA 0012-03 and NACA 0012-103 aerofoils with different nose radii. The blunt aerofoil has larger stagnation region than the sharper aerofoil. In case of blunt aerofoil, the mean velocity gradients are smaller than compared to sharper aerofoil. This causes smaller fluctuation velocities in the case of blunt aerofoil resulting in lower radiation power.

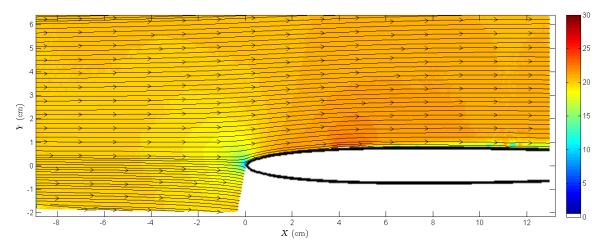

(a) NACA 0006-63

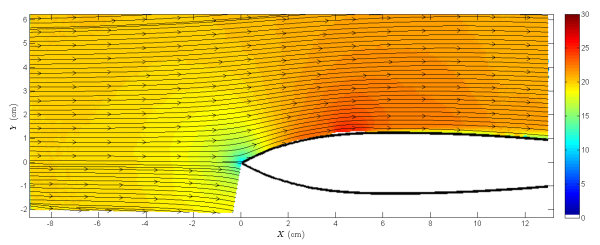

(c) NACA 0012-03

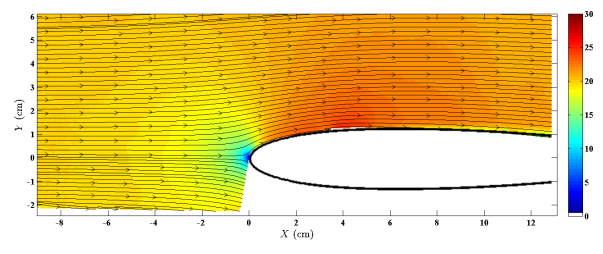

(b) NACA 0012-63

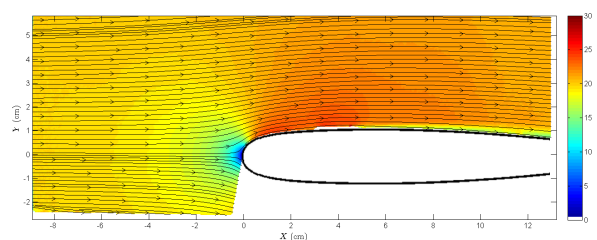

(d) NACA 0012-103

FIGURE 2.25: Mean Velocity maps $(\mathrm{m} / \mathrm{s})$ and streamlines around the aerofoils of varying thickness and nose radius measured using PIV. 


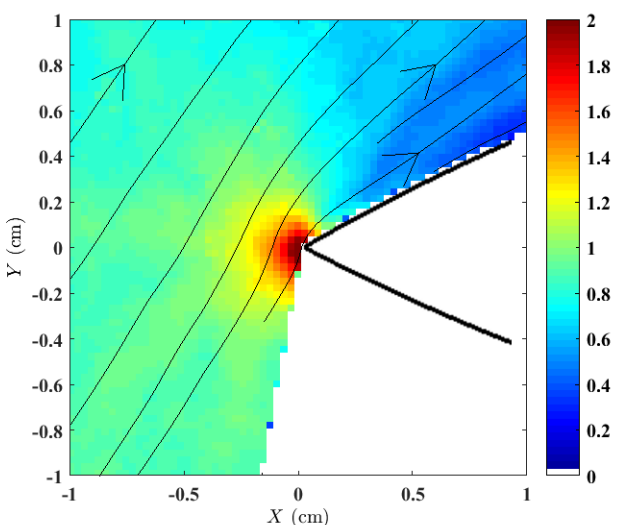

(a) NACA 0012-03

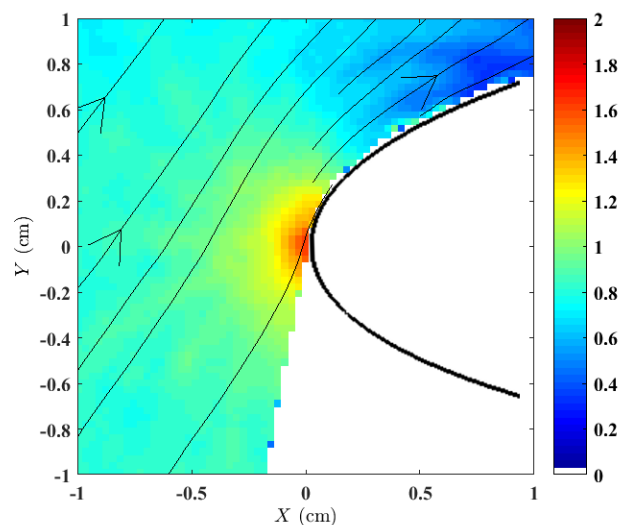

(b) NACA 0012-63

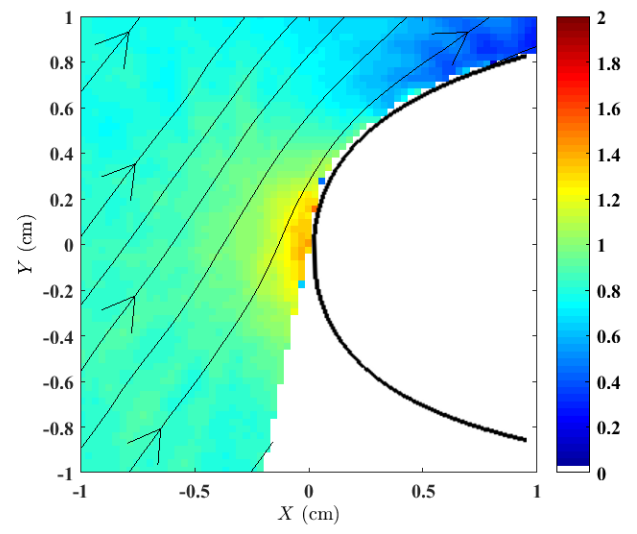

(c) NACA 0012-103

FIGURE 2.26: Fluctuating velocity $\left(v_{y}\right)$ contours $(\mathrm{m} / \mathrm{s})$ and streamlines around the aerofoils measured using PIV.

It is well known from the Amiet's theory that the upwash turbulent velocity $v_{y}$, which is normal to the aerofoil surface, is the responsible component for generating turbulence-aerofoil interaction noise. The velocity fluctuations in the direction normal to the surface of aerofoil are observed close to leading edge of an aerofoil. Figure 2.26 shows the Y-component velocity fluctuations $\left(v_{y}\right)$ for different aerofoil geometries. For comparison, along the tip of the aerofoil in $\mathrm{X}$ direction, fluctuating velocities are plotted along the flow direction for three different nose shape parameters ( $I=0,6$ and 10$)$ for $12 \%$ thick aerofoil. $X=0$ indicates the leading edge tip of the aerofoil. Figure 2.27 shows the comparison between the fluctuating velocities for aerofoils of nose shape parameters $\mathrm{I}=0,6$ and 10. In case of sharp aerofoils, the fluctuating velocities are high at the tip compared to blunt aerofoils. This is one of the reasons for having lower sound power levels for the case of blunt aerofoils. 


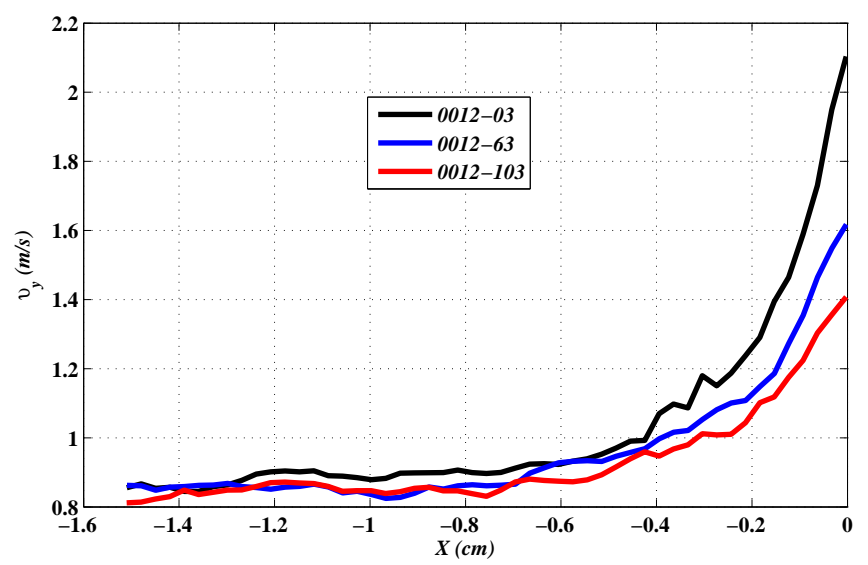

FIGURE 2.27: Comparison of fluctuating velocities $\left(v_{y}\right)$ for different nose radii.

Similar plots have been plotted for aerofoils of varying thickness. It was observed that the thin aerofoils have larger fluctuating velocities as compared to thicker aerofoils, resulting in higher turbulence-aerofoil interaction noise. Both the aerofoil nose radii and thickness change the flow characteristics and greater fluctuating velocities at the tip of the aerofoil results in higher leading edge noise.

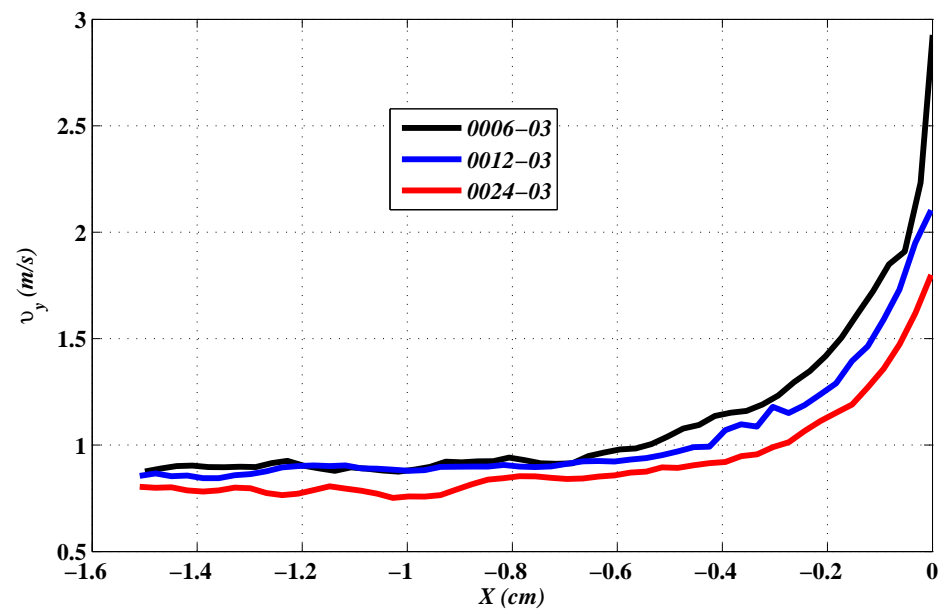

FIGURE 2.28: Comparison of fluctuating velocities $\left(v_{y}\right)$ for different thickness for sharp aerofoils $(\mathrm{I}=0)$.

\subsection{Summary}

In this chapter, a detailed experimental study has been performed to understand the influence of aerofoil geometry on turbulence - aerofoil interaction noise. Systematic noise measurements have been carried out by varying aerofoil thickness and leading edge nose radius separately. Flat plate analytical theory has been shown to be in very close agreement with the measured data. The main findings from this study are: (a) sound power reductions increase with increasing aerofoil thickness $t$, (b) sound power reductions are highly sensitive to the aerofoil nose geometry and radius, (c) sound power reductions for a particular aerofoil geometry are found to follow a Strouhal dependence, (d) sound power reductions 
are most sensitive to nose radius effects for thin aerofoils, and (e) sound power reductions are independent of camber and angle of attack (f) sound power reductions are independent of incoming turbulent integral length scale. It has been made clear that one can't just use a single lengthscale parameter to correct for geometric effects. As the primary mechanism is the mean flow, one has to account for the global geometry of the aerofoil.

The character of the noise and the difference compared to a flat plate have been predicted to be identical at both low and high velocities, suggesting identical noise generation mechanism over the entire velocity range. Compressibility effects associated with high flow speeds are therefore not predicted to introduce additional noise sources. Thus, interaction noise measurements made in low speed wind tunnel provide a valid assessment of noise generation that may be extrapolated to higher flow speeds.

Applying the findings above to a realistic OGV aerofoil of $5 \%$ thickness $(t=3.35 \mathrm{~mm})$ at approach Mach numbers $(M=0.5)$ shows that the non-dimensional frequency $f t / U$ at the third Blade Passing Frequency $(3000 \mathrm{~Hz})$ is approximately 0.06 . At these very small nondimensional frequencies, the influence of aerofoil geometry on turbulence-aerofoil interaction noise has been shown in figure 2.9 to be negligible. These results compels us to investigate alternative methods for reducing interaction noise on thin aerofoils, which is the subject of Chapters $4-7$. However, before this investigation it is important to establish whether the same principle also applies to aerofoils in high solidity cascades where mutual blade interaction is important. This is the subject of the next chapter. 


\title{
Chapter 3
}

\section{Turbulence-Airfoil interaction noise in cascades}

\author{
Contents

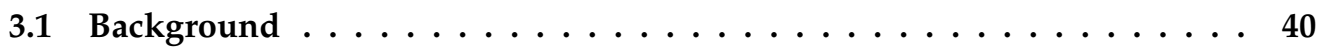 \\ 3.2 Scope of the chapter . . . . . . . . . . . . . . . . 40 \\ 3.3 Fourier description of the radiated sound power from 2D aerofoil cascades 42 \\ 3.4 Numerical Procedure . . . . . . . . . . . . . . . . . . . . . 45 \\ 3.4 .1 Steady flow simulations $\ldots \ldots \ldots \ldots \ldots$ \\ 3.4.2 Linear unsteady CFD simulations . . . . . . . . . . . . . . 46 \\ 3.4.3 Post-processing for upstream and downstream noise . . . . . . . . 46 \\ 3.5 Validation on Flat Plate annular cascade . . . . . . . . . . . 46

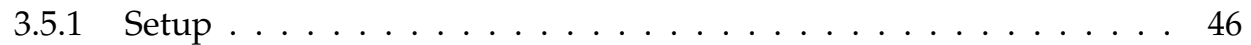 \\ 3.5 .2 Results . . . . . . . . . . . . . . . . . 47 \\ 3.6 Realistic aerofoils . . . . . . . . . . . . . . . 51

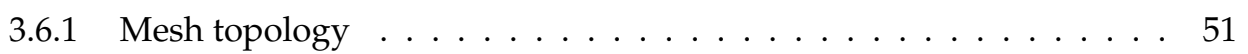 \\ 3.6.2 Typical Mean Flow . . . . . . . . . . . . . . . . . . . . . 52

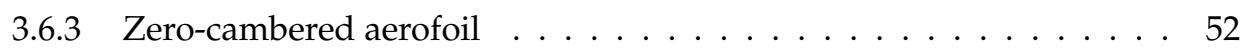 \\ 3.6 .4 Cambered aerofoils . . . . . . . . . . . . . . . . . . 62

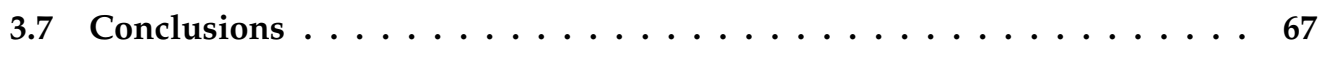

It has been shown in the previous chapter that at realistic mean flow and desirable frequency of interest (3BPF) for modern turbofan engine, isolated aerofoil geometry effects by themselves do not significantly effect broadband noise. In the present chapter we extend our analysis to cascade. This chapter deals with the broadband noise generated due to the interaction of turbulence and a cascade of 2-dimensional realistic aerofoils. The main objectives of this chapter are as follows:

1. To develop theoretical framework by which turbulence-cascade broadband interaction noise can be predicted in 2 dimensions using a linearized Navier-stokes solver and a Fourier turbulence description.

2. To investigate the influence on turbulence-cascade broadband interaction noise due to aerofoil thickness and nose radius.

3. To investigate the effect on turbulence-cascade interaction noise due to camber and flow incidence angle.

First the numerical predictions are validated against LINSUB which provides a numerical solution for the radiated upstream and downstream acoustic pressure due to a 2-dimensional 
cascade of flat plates interacting with $2 \mathrm{D}$ turbulent flow where the mean flow is aligned with the stagger angle. Later, the approach is extended to $2 \mathrm{D}$ realistic aerofoils. The physical aspects of the cascade-gust interaction are studied and discussed. This Fourier approach provides insight into the aerofoil cascade response across the spectrum of vortical mode indices of incoming turbulence, which leads to better understanding of the noise generating mechanism. The influence of aerofoil geometry changes, including to aerofoil thickness, nose radius and camber, and of flow incidence variation on broadband noise are investigated at a highest frequency of interest where broadband noise is dominant in a modern aircraft engine.

\subsection{Background}

Whitehead (1987) developed a FORTAN code, LINSUB, to compute the cascade response of flat plate due to a single frequency, harmonic vortical gust using a collocation technique (see section 1.2.1 for more details). Cheong et al. (2006) used the LINSUB cascade solution to formulate an expression for the broadband sound power spectral density from 2D cascades due to $2 \mathrm{D}$ isotropic turbulence. The efficiency of the computation was considerably improved by exploiting the periodicity of the cascade response function in the transverse turbulence wavenumber which allows re-ordering of the summations over vortical mode indices and scattering indices. This periodicity property is explored in this thesis for cascades of realistic 2D geometries. In this paper, Cheong et al. (2006) derived a simple analytic expression for the radiated sound power that is valid in the high frequency limit.

More recently Polacsek et al. (2015) presented a three-dimensional computational hybrid method aiming at simulating the acoustic modes from a ducted annular cascade subjected to a prescribed homogeneous isotropic turbulent flow. The predicted fluctuating pressures over the aerofoil were used as the input to a Ffowcs Williams and Hawkings integral solver to calculate the radiated sound field. This analysis is limited to plane mode in the azimuthal direction.

In this thesis, aerofoil geometry effects on turbulence-cascade interaction noise is investigated numerically. The numerical approach was then used to show that the periodicity property in the blade response for the flat plate cascade was also sufficiently accurate to be used to allow the modal powers for a realistic aerofoil cascade to be summed infinity by only computing the blade response for modes between 0 and $\mathrm{B}$, where $\mathrm{B}$ is number of blades. It will be shown that flat plates approximation is good assumption to predict the radiated power within $1.5 \mathrm{~dB}$ from an OGVs at the realistic mach number and frequency of interest $(f t / U \leq 0.2$, where $f$ is the frequency of interest, $U$ is the mean velocity and $t$ is the maximum thickness of OGV).

\subsection{Scope of the chapter}

This chapter provides a detailed numerical study into the influence of $2 \mathrm{D}$ aerofoil geometry on the broadband noise due to the interaction between a cascade of $2 \mathrm{D}$ aerofoils and a 2D homogeneous isotropic turbulent flow. The study is restricted to two dimensions to maintain the efficiency of computation. All variations studied here are restricted to changes in 2D geometry, such as aerofoil thickness, nose radius, camber etc, and are therefore not affected by velocity fluctuations in the span-wise direction. As shown by the Amiet flat plate 


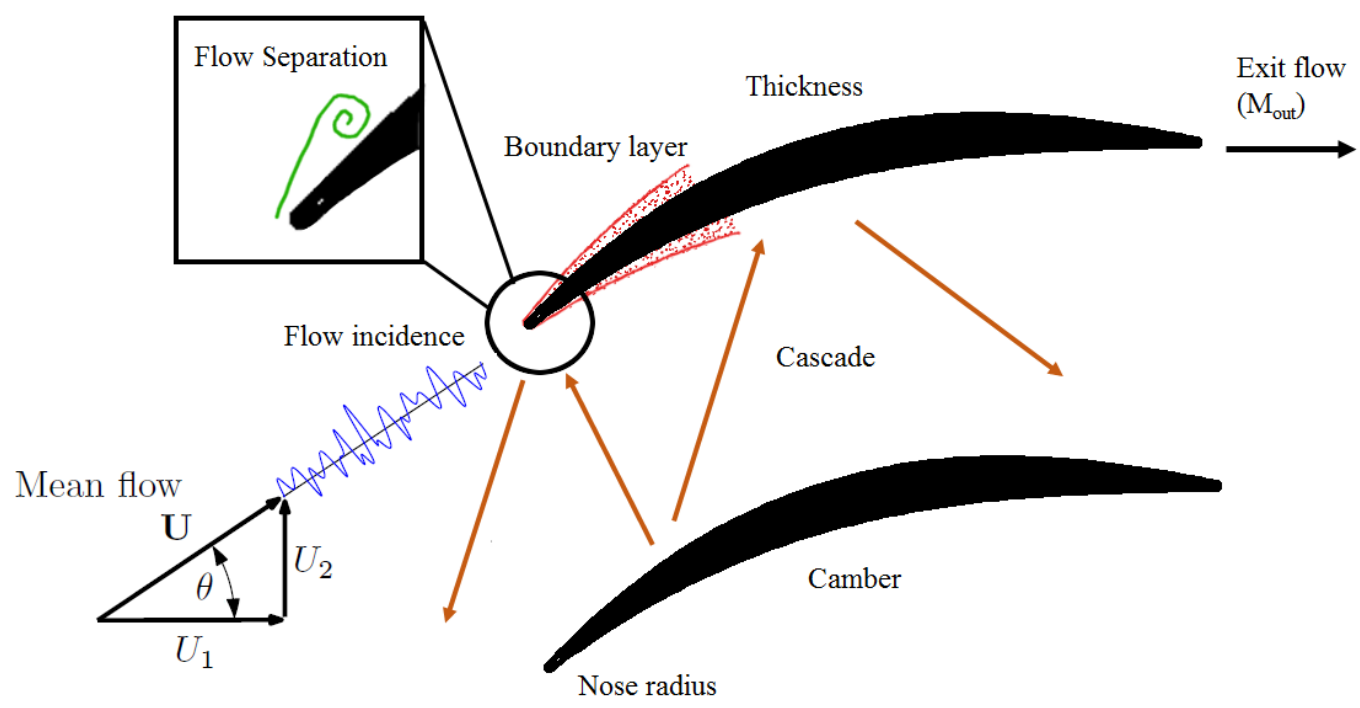

FIGURE 3.1: Schematic description of various possible phenomena effects the radiated downstream and upstream power

theory, for 2D geometries, 3D turbulence simply has the effect of introducing a spanwise correlation along the span but the essential blade response response remains unchanged. The approached described here is based on a decomposition of the turbulent flow into Fourier components of unsteady velocity. The cascade response of each mode is considered in turn.

The chapter first introduces the basic formulation of the radiated noise from a cascade of $2 \mathrm{D}$ aerofoils, developed by analogy with the flat plate cascade solution. The main difference now is that the cascade response is predicted from CFD methods. The method is first used to compute the radiated sound power from a cascade of flat plates and compared against the LINSUB flat plate cascade solution. The chapter proceeds to investigate the effects of aerofoil geometry on broadband noise radiation, such as thickness, nose radius on zero-cambered aerofoils and camber. Flow incidence effects on broadband noise are also studied.

Figure 3.1 shows the schematic description of various possible phenomena which influence the radiated upstream and downstream power. The main difference between cascade models and isolated aerofoil models is, in former case the interference effect between neighboring blades can significantly alter the unsteady response which results in radiated power. The cascade response function captures the occurrence of acoustic modes in the inter-blade space as these modes are coupled with propagation and radiate upstream and downstream of the cascade. The geometry effects such as camber plays a important role in determining the cut-on condition whether the wave will propagate or decay. Due to a change in inlet and outlet flow conditions, upstream and downstream propagated wave characteristics vary. The aerofoil thickness plays a role in radiated power, this can be due to change in unsteady response due to thickness and also due to changes in it's coupling with mode propagating due to reduction in the gap-wise distance between adjacent blades. The nose geometry and flow incidence effect might effect the flow around the leading edge which might results in flow separation at the leading edge of the aerofoil. The influence of all these parameters on radiated power is studied in the present chapter. 


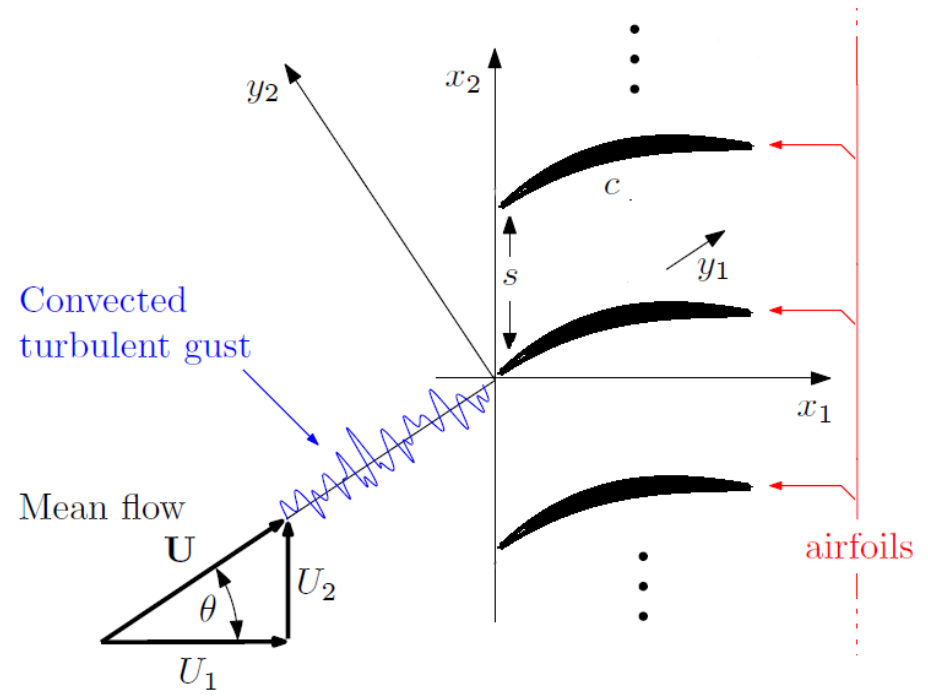

FIGURE 3.2: The convected turbulence gust interacting with cascade of aerofoils

\subsection{Fourier description of the radiated sound power from $2 \mathrm{D}$ aero- foil cascades}

We start with developing the basic equations for predicting the spectrum of acoustic power due to a two-dimensional cascade of aerofoils following the Fourier approach of Cheong et al. (2006).

A two-dimensional cascade of aerofoils with leading and trailing edge angles $\theta_{L E}$ and $\theta_{T E}$ are situated in a two-dimensional uniform mean flow with an incoming flow angle $\theta$ as shown in figure 3.2 .

Consider a single incoming harmonic vortical gust impinging onto the cascade of aerofoils with velocity component $u_{2}$ normal to the flow direction of the form, impinging onto the cascade of aerofoils,

$$
u_{2}\left(y_{1}, y_{2}, t\right)=u_{2} e^{i\left(k_{1} y_{1}-\omega t+k_{2} y_{2}\right)}
$$

The acoustic pressure propagating upstream and downstream of the aerofoil cascade due to the single vortical mode can be expressed as the sum of a number of scattered acoustic modes. In this chapter we define a non-dimensional pressure amplitude $R_{r}^{ \pm}$that is consistent with the amplitude definition for a aerofoil cascade. The upstream and downstream acoustic pressure can therefore be written in the same form as for a flat plate cascade,

$$
p^{ \pm}\left(x_{1}, x_{2}, t\right)=\rho U u_{2} \sum_{r=-\infty}^{\infty} R_{r}^{ \pm} e^{i\left(-k_{1} U t+\alpha_{r} x_{1}+\beta_{r} x_{2}\right)}
$$

where $u_{2}$ is the unsteady velocity amplitude at the blade leading edge normal to the flow direction and $\alpha_{r}$ and $\beta_{r}$ are the axial and circumferential acoustic wave numbers of the $r^{t h}$ acoustic wave The non-dimensional amplitudes $R_{r}^{ \pm}$are readily calculated for a flat plate cascade using, for example, the co-location method developed by Smith and Whitehead or the Wiener-Hopf solution developed by Glegg $(1998,1999)$. However, for a cascade of 
realistic aerofoils, the blade response is a complex function of blade geometry and angle of attack and must generally be computed using CFD methods.

Substituting the form of the acoustic pressure of Eq. 3.2 into the Euler equation yields an expression for the axial acoustic wavenumber $\alpha_{r}$ given by

$$
\alpha_{r}^{ \pm}=\frac{M_{1}\left(\omega / a-M_{2} \beta_{r}\right) \pm \sqrt{\left(\omega / a-M_{2} \beta_{r}\right)^{2}-\left(1-M_{1}^{2}\right) \beta_{r}^{2}}}{1-M_{1}^{2}}
$$

which defines a cuton condition for the $l^{\text {th }}$ acoustic mode give by,

$$
\left(\omega / a-M_{2} \beta_{l}\right)^{2} \geq\left(1-M_{1}^{2}\right) \beta_{l}^{2}
$$

where to ensure periodicity of the acoustic solution over the cascade we must have $\beta_{l}=l / R$, where $l$ is the index of acoustic mode ' 1 ', $R$ is the mean radius of annulus. The lower and upper limits corresponding to the range of propagating acoustic modes is therefore given by $L_{\min }$ and $L_{\max }$. Note that the range of propagating modes is different upstream and downstream due to the change in swirling Mach number.

In the case of 2D turbulent flow, at a single frequency, we consider a spectrum of vortical modes. For simplicity we consider an idealised 2D isotropic homogeneous turbulent flow that is assumed to be frozen and convecting with a mean velocity of $U$. The $2 \mathrm{D}$ wavenumber spectrum for the unsteady velocity component normal to the flow direction can be expressed in terms of its mean square value $\overline{w^{2}}$ and turbulence integral length scale $\Lambda$. In terms of wavenumber components $\left(k_{1}, k_{2}\right)$ oriented in the flow-fixed reference frame, the wavenumber spectrum is of the form:

$$
\Phi_{w w}\left(k_{1}, k_{2}\right)=\int_{-\infty}^{\infty} \Phi_{w w}\left(k_{1}, k_{2}, k_{3}\right) d k_{3}
$$

where

$$
\Phi_{w w}\left(k_{1}, k_{2}, k_{3}\right)=\frac{2 \overline{w^{2}} \Lambda^{5}}{\pi^{2}} \frac{k_{1}^{2}+k_{2}^{2}}{\left(1+\left(k_{1}^{2}+k_{2}^{2}+k_{3}^{2}\right) \Lambda^{3}\right)^{3}},
$$

where for frozen turbulence, we can write

$$
k_{1}=\omega / U \text {. }
$$

In the current CFD method, vortical modes are introduced at the inlet plane in the form of $u_{2 v} e^{i\left(x_{2} k_{2 v}-\omega t\right)}$ where $k_{2 v}$ is the transverse vortical wavenumber, which can be expressed in terms of a modal index $m$ to ensure periodicity of the form

$$
k_{2 v}=m / R
$$

We now use the streamwise wavenumber $k_{1}=\omega / U$ and the transverse vortical wavenumber $k_{2 v}=m / R$ to determine the vortical wavenumbers in the flow-normal direction $k_{2}$ and the duct axial direction $k_{1 v}$, the latter being necessary to deduce the streamwise velocity component $u_{1 v}$ that is consistent with the normal component $u_{2 v}$ in the divergence-free sense. Here, $R$ is the mean radius of the cascade. 
For a frozen gust, $\omega=k_{1} U=\underline{k} \cdot \underline{U}$, where $(\underline{k})=\left[k_{1 v}, k_{2 v}\right]$ and $(\underline{U})=\left[U_{1}, U_{2}\right]$. Resolving $k_{1}$ into its component $k_{1 v}$ and $k_{2 v}$, leads to the following expression for the vortical wavenumber in the duct-axis direction $k_{1 v}$,

$$
k_{1 v}=\left(\omega / a-M_{2} k_{2 v}\right) / M_{1}
$$

The axial velocity amplitude $u_{1 v}$ necessary in the CFD computation, may be obtained from the transverse component $u_{2 v}$ be requiring that the velocity input into the computational domain is divergence free, $\underline{k} \cdot \underline{u}=0$ and hence does not generate pressure by itself. This condition gives,

$$
u_{1 v}=-u_{2 v} \frac{k_{2 v}}{k_{1 v}}
$$

The vortical wavenumber $k_{2}$ necessary in Eq. 3.5 for the turbulence wavenumber spectra may be deduced from the transverse vortical wavenumber $k_{2 v}$ by noting $k_{2} \cos (\theta)+$ $k_{1} \sin (\theta)=k_{2 v}$ and hence,

$$
k_{2}=k_{2 v} / \cos (\theta)-k_{1} \tan (\theta)
$$

Finally, the velocity amplitude $u_{2 v}$ in the duct transverse direction which forms the amplitude of the $m^{\text {th }}$ vortical mode can be computed from

$$
u_{2}=u_{2 v} \cos (\theta)-u_{1 v} \sin (\theta)
$$

where $u_{2}$ is the rms fluctuating velocity normal to the flow direction which can be deduced from Eq. 3.5. The vortical wave definitions $\left(u_{1 v}, u_{2 v}\right)$ in $\left(x_{1}, x_{2}\right)$ direction are imposed on the inlet boundary to solved for unsteady flow field.

To complete the analysis we note that the acoustic mode of order $l$ is due to scattering of the vortical mode of order $m$ and we can therefore write

$$
l=m+B r
$$

where $B$ is number of blades and $r$ is the scattering index.

In the CFD computations presented below, the radiated sound power is computed from the sum of contributions from an infinite number of incoming vortical modes $m$ whose amplitude is determined from Eq. 3.12, where each mode is then, scattered into an infinite number of acoustic modes $l$,

The total radiated sound power due to a cascade of realistic aerofoils can therefore be written as sum of all cut-on acoustic modes as:

$$
P^{ \pm}(\omega)=\sum_{l=l_{\min }}^{l_{\max }} P_{l}^{ \pm}(\omega)=\sum_{m=-\infty}^{\infty} \sum_{r=-\infty}^{\infty} P_{m, r}^{ \pm}(\omega)
$$

where $P_{m, r}^{ \pm}$is the radiated upstream and downstream power for an acoustic mode of $l=$ $m+B r$. The total radiated upstream and downstream sound power can be deduced by 
computing the axial intensity over the duct cross section. In a duct with mean flow velocity vector $\underline{U}$ is given by

$$
P_{m, r}^{ \pm}(\omega)=\frac{1}{2} \int_{S} \operatorname{Re}\left\{\left(p_{m, r}^{ \pm}(\omega)+\rho \underline{u}_{m, r}^{ \pm}(\omega) \cdot \underline{U}\right)^{*}\left(\underline{u}_{m, r}^{ \pm}(\omega)+p_{m, r}^{ \pm}(\omega) \underline{U} / \rho c^{2}\right) \cdot \underline{n} d S\right\}
$$

where $\underline{u}$ is the acoustic particle velocity vector due to the $m^{\text {th }}$ vortical mode of scattering order $r$ and $\underline{n}$ is the unit vector normal along the axial direction.

The single vortical mode response due to a cascade of aerofoil is calculated using the linearized Navier-Stokes solver. At a specific frequency $\omega$ and a fixed vortical mode $m$ and scattering index $r$ the modal components of the acoustic pressure $p$ and particle velocity $\underline{u}$ are extracted for both upstream and downstream acoustic waves at upstream and downstream locations by the use of an eigenmode breakdown of the unsteady flow in the duct.

The total acoustic power radiated by the cascade can be computed from Eq. 3.14, where in practice the summation over $m$ is taken to ensure convergence of the power solution and the limits on the scattering $r$ are restricted to the values for which the acoustics propagate. In practice, at low frequencies, only a few vortical modes and scattering indices are required to capture all propagating acoustic modes. In this two dimensional problem, the number of propagating acoustics modes is proportional to frequency. In the current formulation the blade response function must be computed for all combinations of vortical mode order $m$ and scattering index $r$, leading to a potentially computationally intensive calculation.

In the case of a flat plate cascade, however, the blade response $R_{r}^{ \pm}$is only a function of the inter-blade phase angle $2 \pi m / B$ and is therefore periodic in $m$ with period $B$. This periodicity problem was exploited by Cheong et al. (2006) to reverse the orders of summation in $m$ and $r$ and hence substantially improve the computational effort needed in the noise calculation to give,

$P^{ \pm}(\omega)=\frac{2 \pi \rho M}{\cos \theta} \sum_{m=-\infty}^{\infty} \Phi_{w w}\left(K_{1}, k_{2}\right) \sum_{r=-\infty}^{\infty}\left|R_{r}^{ \pm}\left(K_{1}, k_{2}\right)\right|^{2} \frac{\omega R e\left\{-a \alpha_{r}^{ \pm}+M_{1}\left(\omega+U_{1} \alpha_{r}^{ \pm}+U_{2} \beta_{r}\right)\right\}}{\left|\omega+U_{1} \alpha_{r}^{ \pm}+U_{2} \beta_{r}\right|^{2}}$

where $k_{2}$ is the wave-number in $y_{2}$ direction expressed in vortical mode number ' $\mathrm{m}$ ' in circumferential (gap-wise) direction.

One of the objectives of this chapter is to determine whether the same periodicity assumption can be made for realistic aerofoil geometries at realistic mach number and frequency of interest.

We start with an attempt to validate the CFD prediction method by computing the upstream and downstream radiated sound powers for a cascade of flat plate aerofoils. The results are compared against Eq. 3.15 with the blade response function $R_{m, r}^{ \pm}$computed using the code LINSUB developed by Whitehead (1987) and Smith (1972).

\subsection{Numerical Procedure}

The 3D viscous CFD solver used in the present study is the Rolls-Royce plc code, HYDRA. HYDRA is a suite of non-linear and linear unsteady solvers which uses efficient secondorder edge-based discretisation on unstructured hybrid grid (Moinier (1999); Campobasso 
and Giles (2003)). A 5 step Runge-Kutta algorithm, with an element collapsing multi-grid accelerator algorithm is used iteratively to converge to a steady state solution. Domain decomposition is used to run the solver in parallel on both shared and distributed memory machines.

\subsubsection{Steady flow simulations}

The accurate prediction of the mean flow around the aerofoil is highly crucial to capture the gust distortion due to mean flow gradients around the stagnation region of the aerofoil. In the present study the Reynolds Averaged Navier-stokes (RANS) solver as part of HYDRA is used to obtain the mean flow around the aerofoil geometry. The Spalart-Allmaras turbulence model is used. The limitation of the present scheme is its limited accuracy for separated flows.

\subsubsection{Linear unsteady CFD simulations}

The linear unsteady CFD HYDRA module is based on a full linearisation of the NavierStokes equation. The unsteady problem is solved in the frequency domain for the complex flow amplitudes, $\hat{q}(x)$. A pseudo-time derivative is introduced into the linear equation to enable the same numerical methods adopted for the non-linear CFD code to be used to converge the linear unsteady solution.

$$
q(x, t)=\bar{q}(x)+\hat{q}(x) e^{i \omega t}
$$

Non-reflecting boundary conditions based on an eigen-mode analysis of the unsteady flow field are applied at the inflow / outflow boundaries in order to minimize spurious reflections from the boundary (Moinier and Giles (2005); Moinier et al. (2007)). Further details about the solver and the process are described in Appendix C.

\subsubsection{Post-processing for upstream and downstream noise}

The acoustic data obtained from the unsteady CFD solution is extracted using a wavespitting technique Giacche et al. (2011). Based on the incoming transverse mode numbers, the scattered acoustic cut-on modes are calculated and the upstream and downstream power $P_{m, r}^{ \pm}(\omega)$ calculated from equation 3.15.

\subsection{Validation on Flat Plate annular cascade}

\subsubsection{Setup}

By way of validation the numerical approach outlined above is implemented to predict the noise due to a single vortical mode of arbitrary order $m=40$ impinging upon a nonstaggered flat plate $2 \mathrm{D}$ cascade with $s / c_{0}=0.7, M=0.484$ and reduced frequency $f c_{0} / U=$ 1.88. The predictions are compared against the $2 \mathrm{D}$ solution obtained using the co-location method developed by Whitehead (1987) and Smith (1972) and implemented in the LINSUB code. Figure 3.3 shows the computational domain. The mesh is uniformly spaced except 


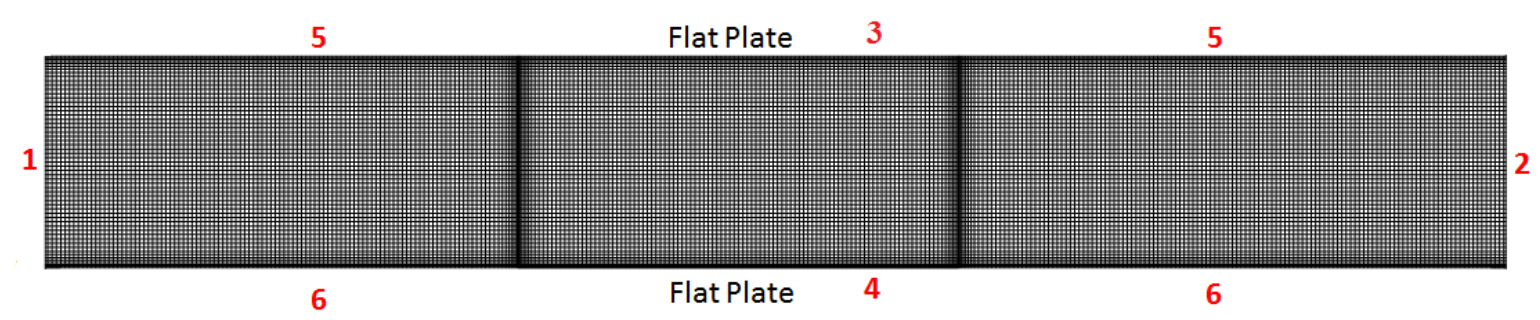

FIGURE 3.3: Flat Plate Grid details showing the grid refinement at leading and trailing edge (Boundary conditions: 1-subsonic inflow; 2-subsonic outflow; 3,4-Inviscid walls; 5,6-Periodic boundary)

at the leading and trailing edges of the flat plate. At the reduced frequency of 1.88 used in this validation test, 70 points per wavelength $a / f$ and 140 points close to trailing and leading edges are used. The mesh is sufficiently fine to ensure a converged solution of all cut-on acoustic modes. The grid convergence study has been demonstrated in Appendix C. A quantitative mesh refinement study has been carried out to quantify the errors due to dissipation and dispersion.

The simulations assume a mean flow speed of $(M=0.48)$ which is initiated uniformly across the passage. The cascade is simulated by introducing flat plates either size of the passage as shown in figure 3.3 and imposing a periodic boundary condition on surfaces $5 \& 6$. Other boundary definitions are shown in figure 3.3. The vortical wave mode is imposed at the inlet boundary of the domain with unit velocity amplitude. As LINSUB is an inviscid theory, all surfaces in the domain are described by an inviscid wall boundary condition.

By way of further validation, a second flat plate case was studied with stagger angle in which similar grid definitions are used. The grid is skewed relative to the direction of propagation and due to skewness a small amount of dissipation is introduced as it depends on the alignment of the grid and the wave propagation.

\subsubsection{Results}

\section{Single incoming mode validation}

Figure 3.4a and $\mathrm{b}$ shows the results of the instantaneous axial velocity and unsteady pressure respectively. The presence of modes within the cascade are clearly seen. From Eq. 3.13, for a incoming mode $m=40$, the inter blade phase angle is $2 \pi \times 40 / 44$. The angular frequency of the simulation was set at $\omega=30000 \mathrm{rad} / \mathrm{s}$, which is below the frequency of $40000 \mathrm{rad} / \mathrm{s}$ at which the first cascade mode cuts on. Figure 3.4a shows that in this example of zero stagger angle the vortical mode passes through the cascade with apparently little effect on particle velocity. The pressure variation in Figure 3.4b however shows evidence of modal behaviour within the cascade, which does not appear to be effectively transmitted into the 'duct'. According to the scattering rule of Eq. 3.13 the combination of $m$ and $B$ gives rise to acoustic mode, $l=-4$ propagating upstream and downstream in the duct. This mode is clearly seen in figure $3.4 \mathrm{~b}$ which is of lower order that varies slowly over the a single cascade passage.

Further validation of the prediction method is provided in figure 3.5a and $b$ which shows a comparison between the CFD and LINSUB predictions of the upstream and downstream acoustic pressure amplitudes versus frequency $f c_{0} / U$ between 0.25 to 2 for a fixed incoming transverse mode number $m=40$, of unit upwash $u_{2 v}=1$ and a mean flow Mach number 


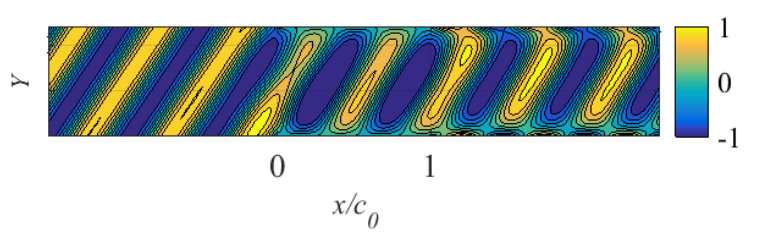

(a) Instantaneous Axial Velocity

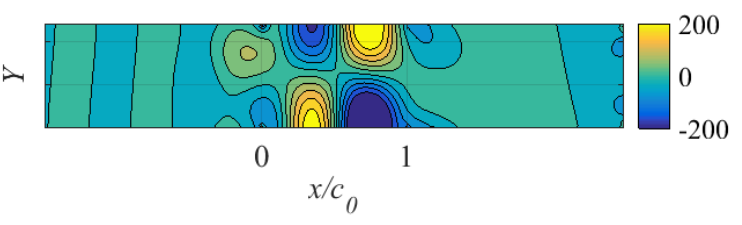

(b) Instantaneous Pressure

FIGURE 3.4: Unsteady Contours on zero-staggered Flat plate for incoming transverse mode $\mathrm{m}=40$ at $\mathrm{M}=0.48$

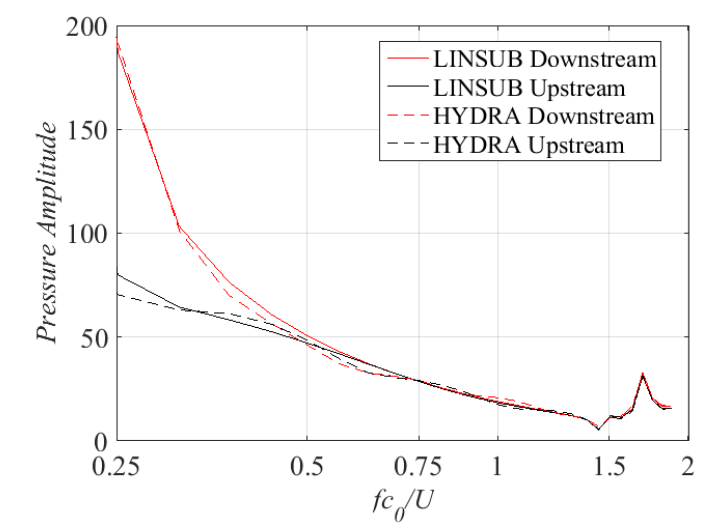

(a) Staggered angle, $\theta=0$, Incoming transverse mode $m=$ $40, M=0.484$

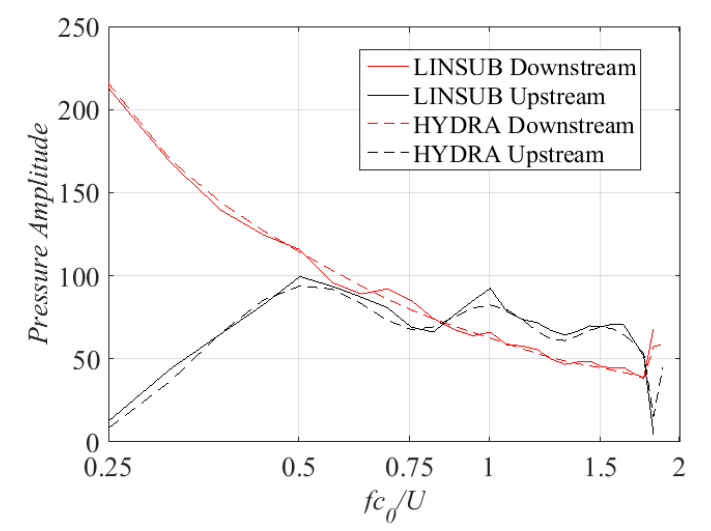

(b) Staggered angle, $\theta=40$, Incoming transverse mode $m=$ $40, M=0.484$

FIGURE 3.5: Flat plate numerical results validated against LINSUB

of 0.484 . Results are shown for zero-stagger angle and a $40^{\circ}$ stagger case, where agreement within a few percent is obtained over the frequency range. Grid alignment studies for the stagger case are discussed in Appendix C.

Comparisons are now made between the CFD and LINSUB predicted pressure-difference amplitudes across the flat plates for a transverse mode number $m=40$ at angular frequency $\omega=30000 \mathrm{rad} / \mathrm{s}, M=0.484$ and stagger angle of 0 . The variation in pressure difference across the chord is plotted in figure 3.6a and a plot of the same data zoomed in around the leading edge shown figure 3.6b. Again, agreement is better than a few percent across the entire chord, particularly near the leading edge where the response is greatest.

\section{Multiple incoming modes at fixed frequency}

The comparison between the CFD sound power predictions obtained from Eq. 3.15 and the LINSUB sound power predictions obtained from Eq. 3.16 are now extended to a 2D homogeneous turbulent flow with upwash velocity wavenumber spectra derived from Eqs. 5-11 for a turbulence intensity of $2.5 \%$ and an integral length scale of $\Lambda=0.007$ for a zerostagger flat plate cascade. The predictions are obtained at a fixed angular frequency of $\omega=$ $30000 \mathrm{rad} / \mathrm{s}$ and incoming transverse mode numbers $m$ between 0 and 44 . For mode orders greater than 44 the cascade response for a flat plate is predicted to be periodic, as verified in a later section. Figure 3.7 shows the comparison of sound power due to each incoming mode $m$ obtained by summing the sound powers $P_{m, r}^{ \pm}$over all scattered acoustic modes $r$. 


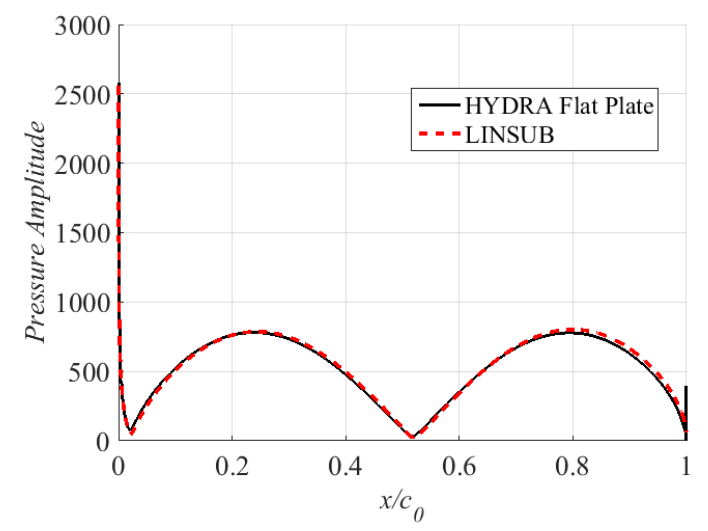

(a)

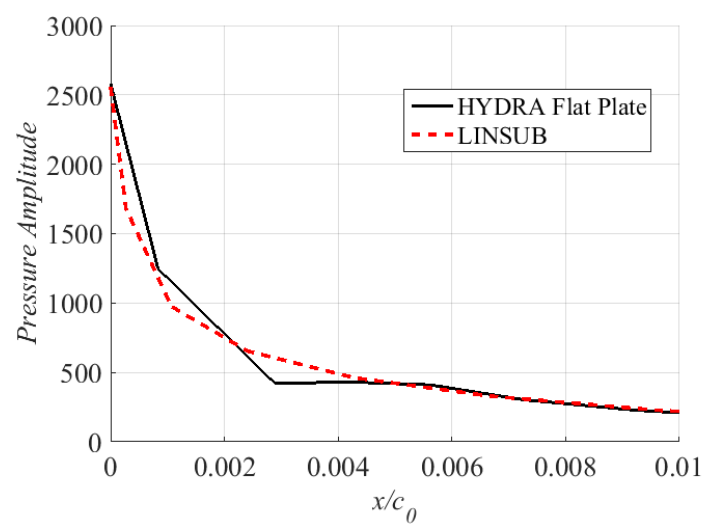

(b)

FIGURE 3.6: Comparison of unsteady pressure jump against LINSUB for incoming transverse mode $m=40$ at $\mathrm{M}=0.48$ and reduced frequency $f c_{0} / U=$ 2.4

The HYDRA and LINSUB predictions are seen to be within $0.5 \mathrm{~dB}$. The largest discrepancy between the two predictions is observed at $m=11$ and 33 whose scattered acoustic modes have a cuton frequency very close to the excitation frequency of $\omega=30000 \mathrm{rad} / \mathrm{s}$. Note that there is no radiated power upstream and downstream for $m=0$ and 44 in this zero stagger angle case. This is because for these mode orders the phase difference between the blades are integral multiples of $2 \pi$ and hence adjacent blades are excited in phase but the upper and lower surface radiated out of phase leading to zero far field radiation. The reason for the small discrepancy of up to $0.5 \mathrm{~dB}$ between the LINSUB and HYDRA predictions in figure 3.7 is currently unknown but may be related to the effectiveness of the non-reflective boundary condition and numerical issues relating to convergence of the solution at the frequencies for which there are acoustical modes close to cut-on.

\section{Validation of periodicity}

Previous work presented by Cheong et al. (2006) has shown how the broadband noise calculation for a flat plate cascade may be made more efficient than Eq. 3.16 by exploiting the periodicity in the blade response function $R_{r}^{ \pm}$since it is only a function of inter-blade phase angle in addition to $s / c_{0}$, stagger angle, Mach number and reduced frequency $\omega c_{0} / U$. We now confirm this periodicity property in the CFD solution.

Figure 3.8 shows the radiated power $P_{m}^{ \pm}$versus $m-r B$, for the two values of the $r=0$ and 1. As expected, the two spectra for $r=0$ and 1 overlap extremely well thereby validating this useful periodicity property predicted by flat plate theory. The validity of this property for a typical OGV aerofoil will be investigated in section 3.6.3.

\section{Broadband Validation}

Finally, we conclude this validation phase by comparing broadband sound power spectra obtained from CFD and summing over sufficient numbers of vortical modes to ensure convergence ( $\approx 132$ in this case) with the efficient solution developed by Cheong with periodicity assumed (Eq. 25 in Cheong et al. (2006)). The CFD spectra plotted in Figure 3.9 


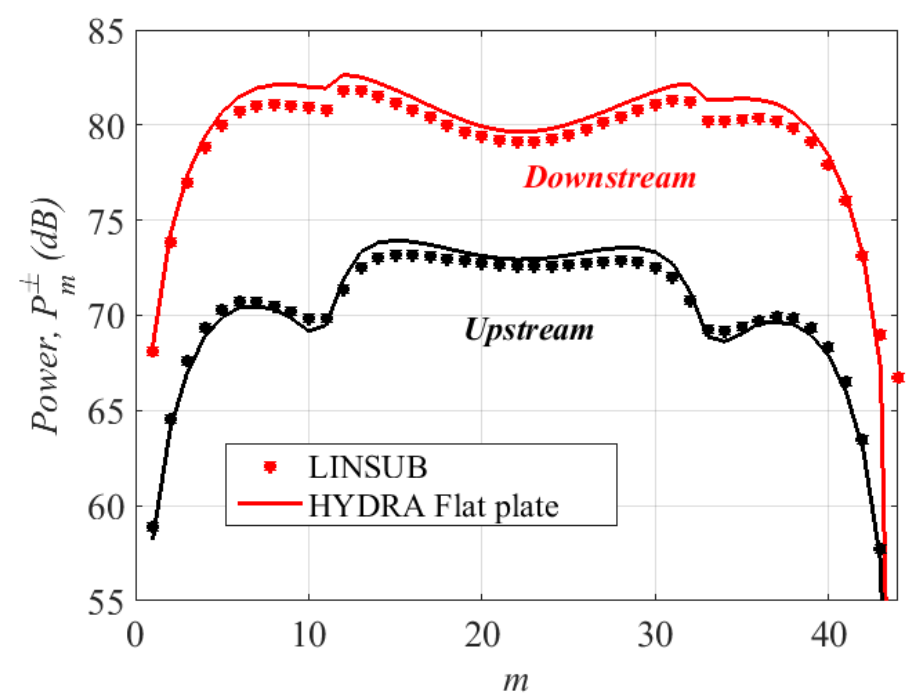

FIGURE 3.7: Flat plate numerical results validated against LINSUB for various incoming transverse mode number at a fixed frequency $\omega=30000 \mathrm{rad} / \mathrm{s}$, $\mathrm{M}=0.484, \theta=0^{\circ}, T I=2.5 \%, \Lambda=7 \mathrm{~mm}$

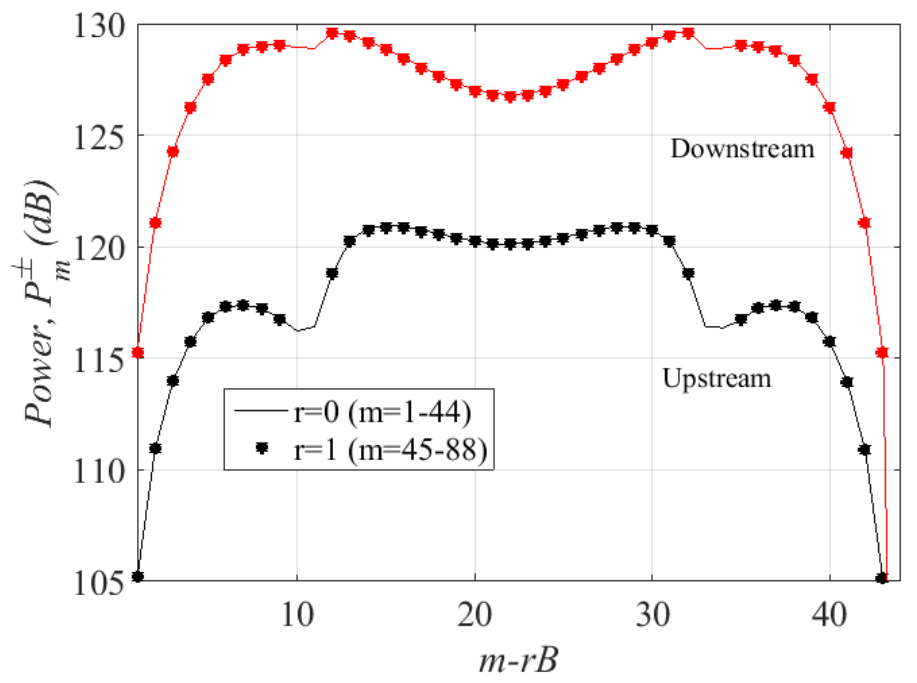

FIGURE 3.8: Radiated power due to various incoming transverse mode numbers at a fixed frequency $\omega=30000 \mathrm{rad} / \mathrm{s}$ and unit normal vortical velocity to flat plate 


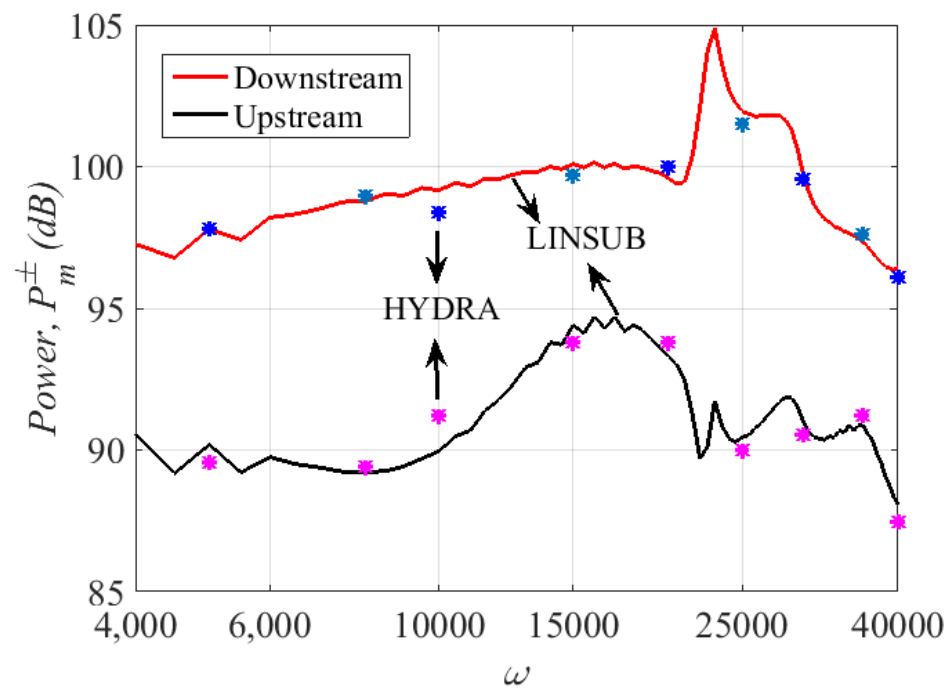

FIGURE 3.9: Overall radiated power across various frequencies at incoming turbulence intensity, $T I=2.5 \%$, and integral length scale $\Lambda=7 \mathrm{~mm}$

are limited to ten discrete frequencies over the frequency range $\omega$ range of 5000 to 40000 and compared to the LINSUB solution with a much finer frequency resolution is around $1 \mathrm{~dB}$. This difference is due to numerical issues relating to convergence of solution the frequencies for which there are acoustical modes close to cut-on.

The numerical CFD approach described above and validated for a flat plate cascade is now used to investigate the effect on broadband noise due to changes in aerofoil geometry such as blade thickness, nose radius, camber and attack angle.

\subsection{Realistic aerofoils}

\subsubsection{Mesh topology}

We now consider a cascade of 2D zero-stagger symmetric aerofoils of $5 \%$ thickness relative to the chord. The location of maximum thickness is at the aerofoil mid-chord.

The mesh generation is performed using the Rolls-Royce in-house software PADRAM (Shahpar and Lapwroth (2003)). An H-O-H mesh topology is used to grid a single OGV passage with the cascade effect simulated by imposing a periodic boundary condition at the upper and lower walls (4 and 5), as shown in Figure 3.10. Also shown are the inlet and outlet boundaries (1 and 2) and the viscous wall boundary (3) on the aerofoil surface. The mesh uses a structured multi-block arrangement with a O-mesh of quadrilaterals cells around the OGV surface and $\mathrm{H}$-meshes above and below the O-mesh and in the inlet and outlet flow region. The mesh density is taken to be sufficiently fine such that the HYDLIN code captures all the propagating cut-on acoustic modes. A total of 31472 nodes are considered for this grid and this is reasonable good for lower transverse mode numbers as the number of nodes in transverse direction are 80 . The boundary condition on respective surfaces are also described in the same figure. Similar mesh topology is used for staggered configuration. 


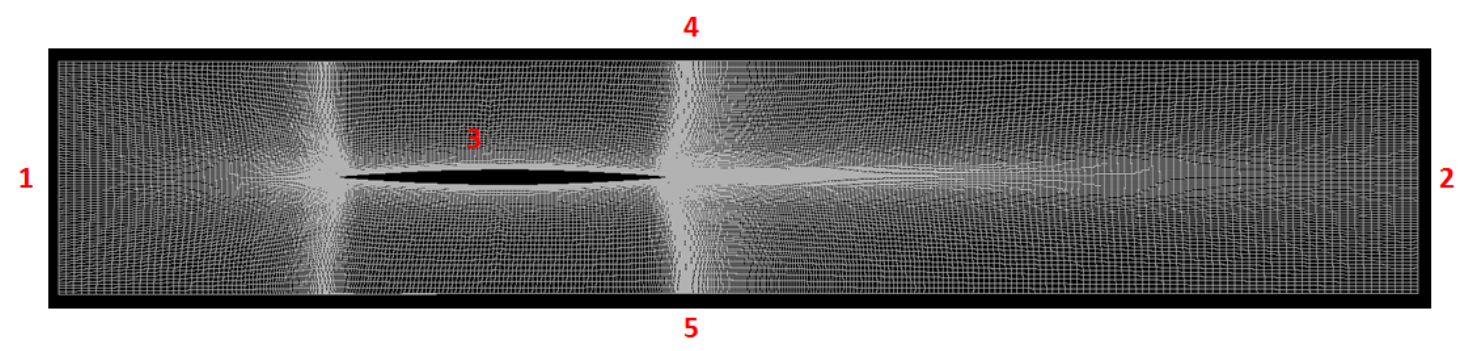

FIGURE 3.10: OGV Grid details showing the grid refinement at leading and trailing edge (Boundary conditions: 1-subsonic inflow; 2-subsonic outflow; 3-Viscous wall; 4,5-Periodic boundary)

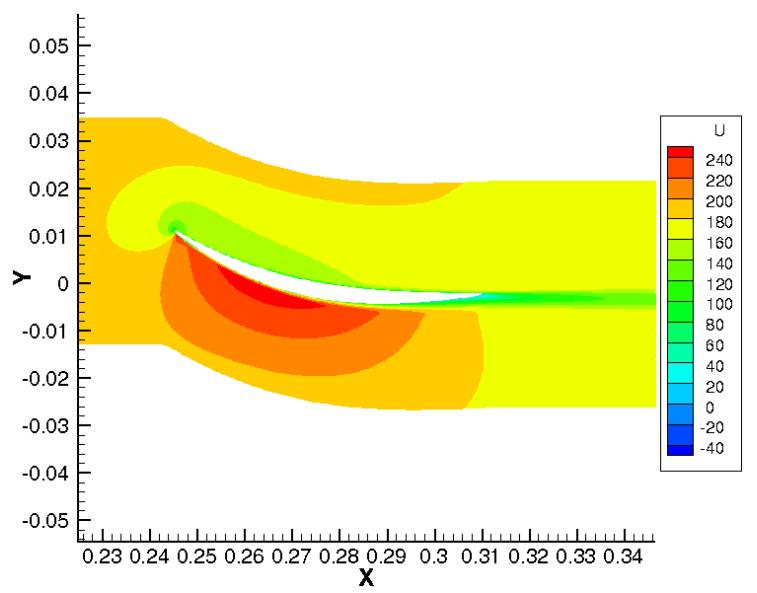

(a) Velocity in the $\mathrm{x}$-direction, between -40 and $240 \mathrm{~m} / \mathrm{s}$

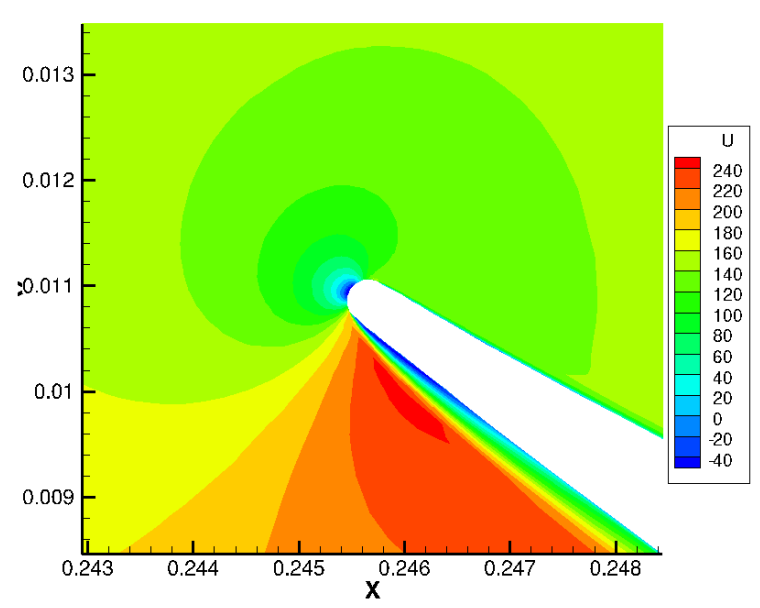

(b) Velocity in the $x$-direction, between -40 and $240 \mathrm{~m} / \mathrm{s}$

FIGURE 3.11: RANS on cambered OGV configuration

\subsubsection{Typical Mean Flow}

Subsonic inlet and outlet conditions are imposed on the inlet and outlet boundary surfaces of the domain. The mean flow Mach number is set at 0.484 and the Spalart-Allmaras turbulence model is used.

Figure 3.11 shows the steady mean flow velocity in stream-wise direction for cambered OGV configuration. One can see the presence of stagnation region close to leading edge in figure $3.11 \mathrm{~b}$.

\subsubsection{Zero-cambered aerofoil}

In this non flat plate case the mean flow was computed using the RANS solver within HYDRA. HYDLIN is then used to perform the linear unsteady aerofoil response calculations by superposing an incoming vortical wave on the mean flow solution. The transverse wavenumber deduced from $m$ and the amplitude $u_{1 v}$ and $u_{2 v}$ is specified on the inlet boundary for each vortical mode. There is no swirl component in the zero-stagger case under consideration here. 


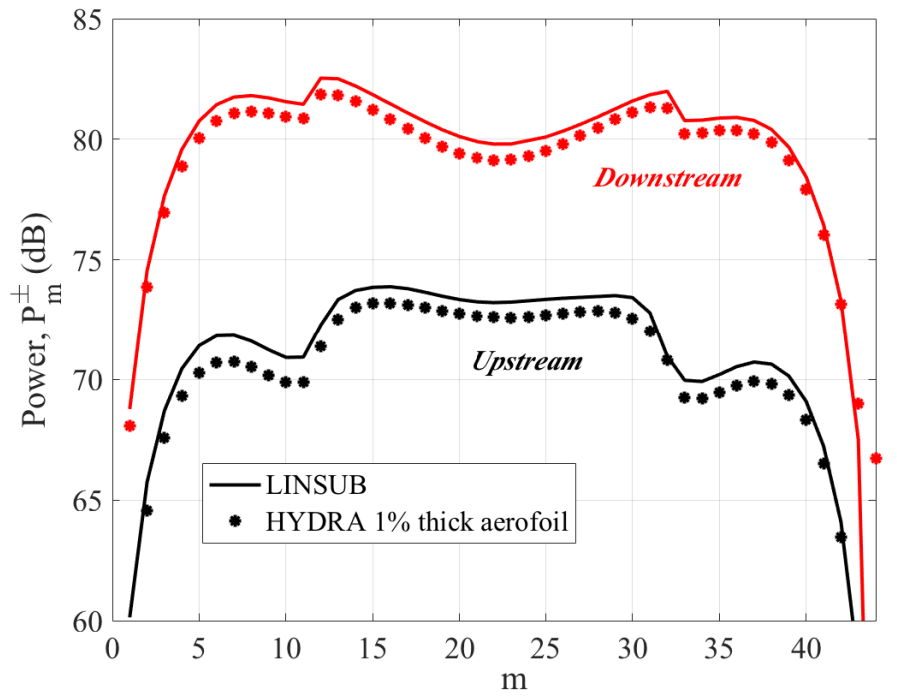

FIGURE 3.12: Comparison of radiated power $P_{m, r}^{ \pm}$between aerofoil (1\% thickness) against LINSUB for various incoming transverse mode number at a fixed frequency $\omega=30000 \mathrm{rad} / \mathrm{s}, T I=2.5 \%, \Lambda=7 \mathrm{~mm}$

\section{Comparison with Flat plate LINSUB solution}

In an attempt to quantify the effects of blade geometry and now viscous mean flow on broadband noise, HYDRA was used to compute the sound power spectrum versus $m$ for a zero-cambered aerofoil and a flat plate cascade. The results are compared against the flat plate analytical solution, LINSUB. The calculations were performed at a fixed frequency $\omega=30000 \mathrm{rad} / \mathrm{s}$ for a range of incoming transverse mode numbers between 0 and 44 . The comparison between upstream and downstream mode power spectra $P_{m}^{ \pm}$is plotted in Figure 3.12.

The total radiated upstream and downstream sound power predicted for the aerofoil cascade with viscous mean flow (CFD-based) are compared against LINSUB solution in Figure 3.12 and the differences of up to $2 \mathrm{~dB}$ are observed. These differences arise from the combined effects of viscosity, which affects flow separation and boundary layer effects in the vicinity of the leading edge, and the effects of blade geometry. We investigate these two effects separately to attempt to quantify their relative importance to broadband noise.

\section{Influence of viscous mean flow on aerofoil noise generation}

In this section the influence of viscous mean flow on noise generation are studied for the case of the aerofoil cascade. Figure 3.13a shows the comparison of the radiated upstream and downstream sound power with incoming transverse mode numbers between 0 and 44 for the aerofoil cascade predictions with viscosity included and when the flow is assumed to be inviscid. The effects of viscous mean flow on the predicted sound power mode spectrum for the aerofoil, are significantly less than for the flat plate. Differences between the two solutions are within $0.5 \mathrm{~dB}$. However, larger differences are observed for upstream propagating modes generated by $m \approx 11$, that scatter acoustic modes with cut-on frequency close to the excitation frequency and for the higher order modes modes $m \geq 33$. 


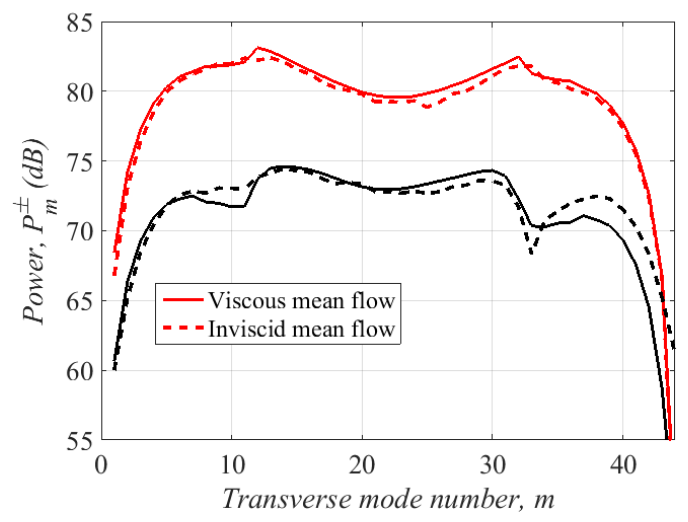

(a) Comparison of radiated power

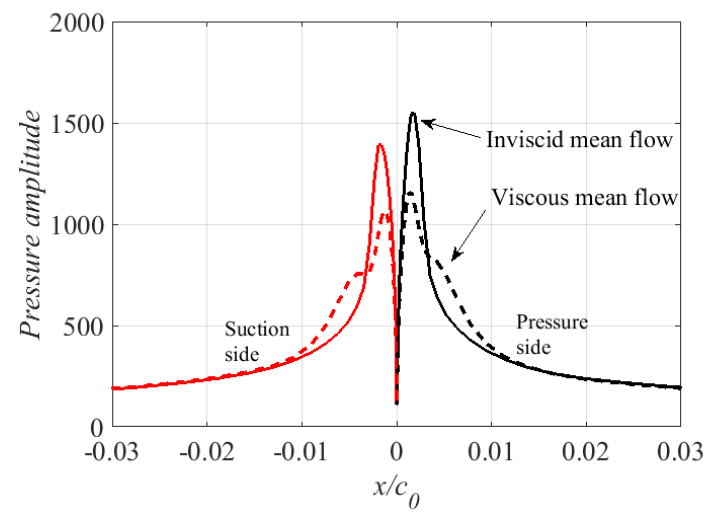

(b) Comparison of unsteady pressure

FIGURE 3.13: Influence of viscous mean flow on $5 \%$ thickness aerofoil at a fixed frequency $\omega=30000 \mathrm{rad} / \mathrm{s}, T I=2.5 \%, \Lambda=7 \mathrm{~mm}$

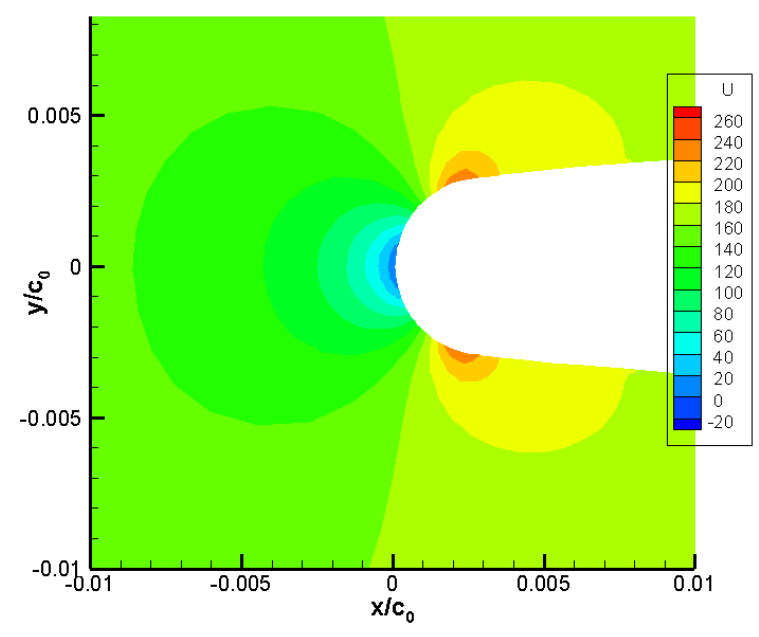

(a) Inviscid mean flow

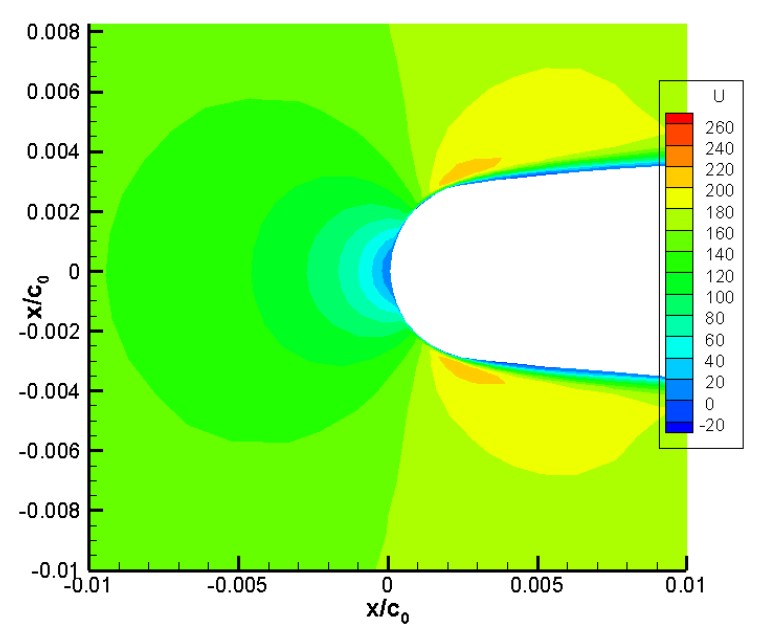

(b) Viscous mean flow

FIGURE 3.14: Axial mean velocity around the leading edge of the aerofoil

One explanation for this finding is shown in figure $3.13 \mathrm{~b}$ which depicts the variation in surface pressure amplitude across the aerofoil with and without viscosity. The increase in sound power associated with the viscous solution is consistent with the small increase in the magnitude in the unsteady pressure amplitude over the surface, plotted in figure $3.13 \mathrm{~b}$, in which the suction side response is plotted for positive $x / c_{o}$ and the pressure side for negative $x / c_{o}$. In this figure, the effects of viscosity can be seen to have the dual effects of reducing the response exactly at the leading edge and also to broaden the response to further downstream due to mild flow separation, as shown in the mean flow axial velocity contours in figure 3.14. The combined effect from these two changes is a small increase in the integrated unsteady lift, leading to a small increase in radiated sound power.

However, we introduce a note of caution since at the present time it is unclear whether the differences of $0.5 \mathrm{~dB}$ are within the accuracy of the numerical approach. In the case of the aerofoil there remains some uncertainties surrounding the convergence of the inviscid mean flow solution. 


\section{Modal convergence}

In the Fourier approach outlined in section 3.3 the total radiated sound power is obtained by summing the contribution from all vortical modes. In this section we investigate whether the periodicity property observed for a flat plate cascade in which the response function $R_{r}^{ \pm}$ is periodic in $m$ with period $B$ is also valid for realistic aerofoils. This property will allow the contribution from all modes to be summed to infinity from only the response function computed for mode orders $m$ between 0 and $B$ (Cheong et al. (2006)).

To test this periodicity property the sound power due to each vortical mode $m$ was computed for a range of vortical mode numbers between 0 to 132, corresponding to an upper limit of $m=3 B$, for the aerofoil cascade at zero-stagger angle (i.e., no swirl). For higher mode numbers, the mesh size in the transverse direction is further decreased to reduce errors due to dissipation and dispersion. Figure 3.15a is a plot of the sound power mode spectrum for the 2D isotropic turbulence velocity spectrum of Eq. 3.5 for a turbulence intensity of $2.5 \%$ and an integral length scale of $7 \mathrm{~mm}$.

The data plotted in figure 3.15a is plotted again in figure $3.15 \mathrm{~b}$ but now normalised to the velocity spectrum so that now only the blade response is being shown. The three periods $m=1: B, m=B+1: 2 B$ and $m=2 B+1: 3 B$ and overlayed to test in detail the periodicity property.

The modal power spectrum in figure $3.15 \mathrm{~b}$ is observed to be periodic to within the accuracy of the CFD solution except for the modes $m=11$ whose acoustic modes are close to cuton and for the higher order modes and $m \geq 33$ when the transverse wavelength becomes comparable with the aerofoil thickness. For $m>120$, the phase difference across the aerofoil thickness $m t / R \geq 1$, where $R$ and $t$ are the duct radius and aerofoil thickness, which results in deviation from periodicity.

Figure $3.15 \mathrm{~b}$ shows that the influence of aerofoil geometry on the mode spectrum is only significant for the for higher order transverse modes. However, their velocity contribution to the overall turbulence velocity is relatively small, as specified by the wavenumber spectrum of Eq. 3.5. Their contribution to the total sound power is therefore negligible in this case.

The cumulative total radiated upstream and downstream sound power obtained by summing all modes from $m=0$ to $m=m_{\max }$, with $m=m_{\max }$ varying from 0 to 220 with periodicity assumed is plotted in figure 3.16. Also shown in this figure as ${ }^{\prime} *^{\prime}$ at $m=m_{\max }=132$ is the sound power prediction obtained by summing the power due to all modes from 0 to 132 without the assumption of periodicity. The predictions are almost identical thereby validating the periodicity approximation for the case of thin aerofoils. This test has also been undertaken for staggered, cambered and thicker aerofoils and shown to be equally valid. From hereon, therefore, all predictions will be made based on the assumption of a periodic cascade response function.

Having verified the periodicity assumption we are now able to compare in Table 3.1 predictions at a single frequency $\omega=30000 \mathrm{rad} / \mathrm{s}$ the total sound power radiation between a flat plate and an aerofoil with and without viscosity effects included. As seen previously viscous effects are seen to be significant for the aerofoil cascade due to change in mean flow around the leading edge of the aerofoil. In view of the significance of viscosity effects for aerofoils, all predictions presented from here on will include viscosity. 

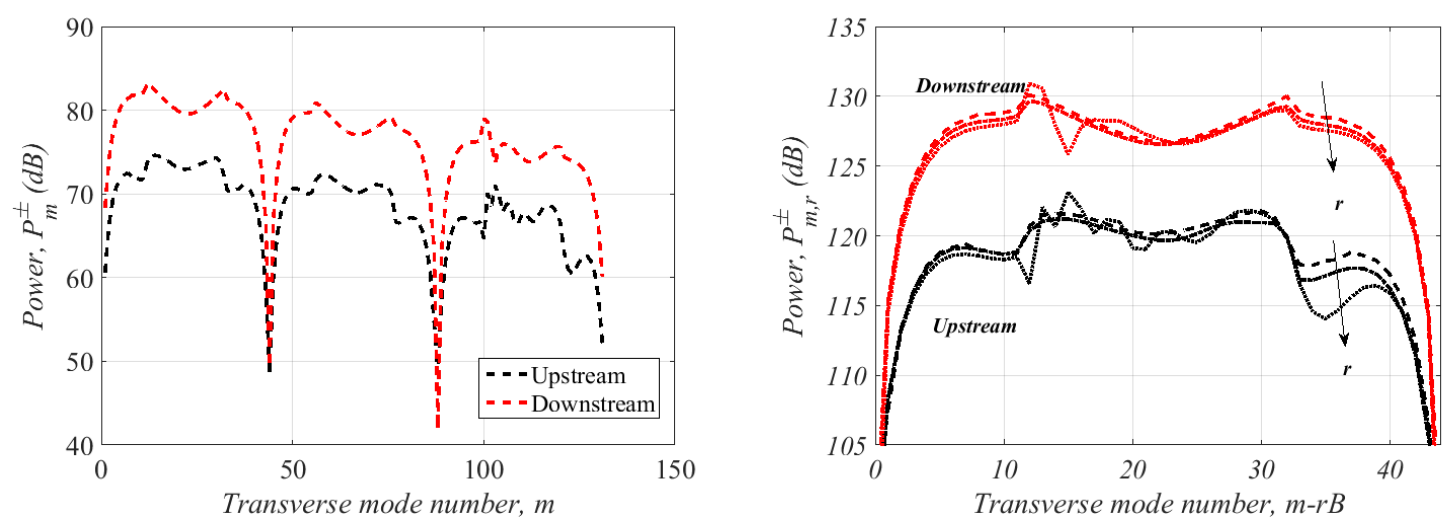

(a) Radiated power for various incoming transverse modes $\mathrm{m}$ (b) Validating periodicity by scaling to unit normal vortical scaled to $T I=2.5 \%, \Lambda=7 \mathrm{~mm}$ velocity to aerofoil

FIGURE 3.15: Radiated power due to various incoming transverse mode numbers at a fixed frequency $\omega=30000 \mathrm{rad} / \mathrm{s}$

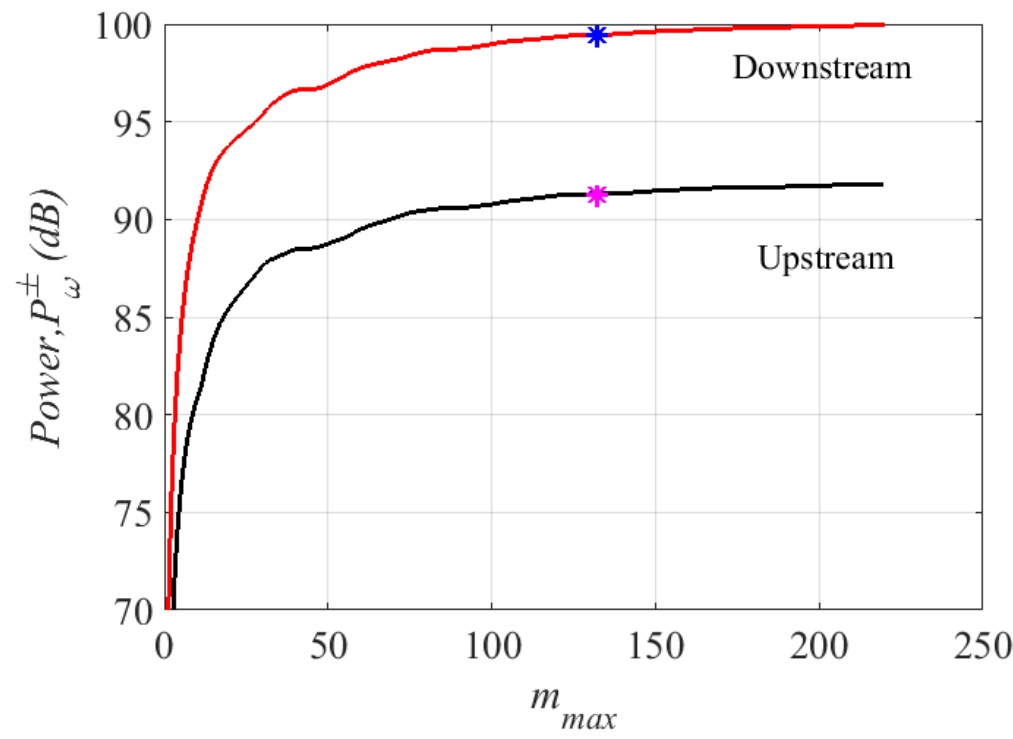

FIGURE 3.16: Convergence of total power level across all incoming transverse mode numbers at a fixed frequency $\omega=30000 \mathrm{rad} / \mathrm{s}, T I=2.5 \%, \Lambda 7 \mathrm{~mm}$, Solid line: Using periodic response, ${ }^{*}$ : Using non-periodic response 


\begin{tabular}{c|c|c}
$\begin{array}{c}\text { aerofoil } \\
\left(t / c_{0}, \%\right)\end{array}$ & $\begin{array}{c}\text { Upstream Power } \\
(\mathrm{dB})\end{array}$ & $\begin{array}{c}\text { Downstream Power } \\
(\mathrm{dB})\end{array}$ \\
\hline \hline aerofoil (Viscous) & 91.7 & 99.94 \\
\hline aerofoil (Inviscid) & 91.8 & 99.61 \\
\hline
\end{tabular}

TABLE 3.1: Influence of viscous effects
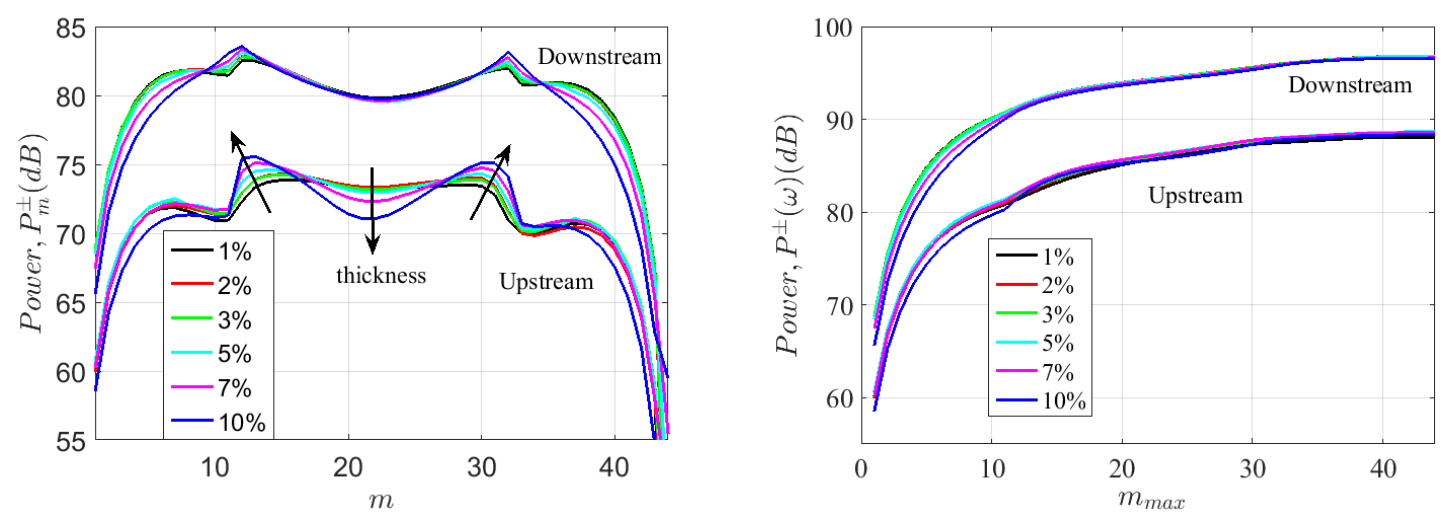

(a) Radiated power for various incoming transverse modes $\mathrm{m}$ (b) Convergence of total power level across all incoming transverse mode numbers

FIGURE 3.17: Influence of aerofoil thickness at a fixed frequency $\omega=$ $30000 \mathrm{rad} / \mathrm{s}, T I=2.5 \%, \Lambda=7 \mathrm{~mm}$

\section{Influence of aerofoil thickness on interaction noise}

\section{Effect of aerofoil thickness on zero-staggered aerofoil}

In this section an investigation into the influence of aerofoil thickness on broadband noise radiation at a fixed frequency $\omega=30000 \mathrm{rad} / \mathrm{s}$ (3rd BFP at Approach conditions). This frequency is chosen to correspond to the upper frequency limit of interest at which changes in noise due to geometry (Thickness and nose radius) are greatest in the desired frequency range. Six sound power spectra are presented in figure 3.17a illustrating the effects on noise due to variations in aerofoil thickness between $1 \%$ to $10 \%$. Variations in modal power are observed of up to $3 \mathrm{~dB}$ are observed for some modes, particularly for those generating acoustic modes close to cut-on. However, when summed over all modes (assuming periodicity), as shown in figure $3.17 \mathrm{~b}$ showing the cumulative sum, the overall sound power variation due to thickness is negligible at this relatively high frequency. The cumulative sum of the downstream and upstream powers for different thickness aerofoils are as summarised in Table 3.2. The variation of the total power is observed to be less than $1 \mathrm{~dB}$. Thus, single vortical modes are significantly affected by blade thickness effects but when summed across all modes representative of turbulence excitation, the blade response is negligible. Precisely this finding was obtained by Evers and Peake (2002) in an analytic study of blade geometry effects on noise. 


\begin{tabular}{c|c|c}
$\begin{array}{c}\text { aerofoil thickness } \\
\left(t / c_{0}, \%\right)\end{array}$ & $\begin{array}{c}\text { Upstream Power } \\
(\mathrm{dB})\end{array}$ & $\begin{array}{c}\text { Downstream Power } \\
(\mathrm{dB})\end{array}$ \\
\hline \hline LINSUB & 91.05 & 99.82 \\
\hline $1 \%$ & 91.20 & 99.88 \\
\hline $2 \%$ & 91.46 & 99.91 \\
\hline $3 \%$ & 91.50 & 99.90 \\
\hline $5 \%$ & 91.78 & 99.94 \\
\hline $7 \%$ & 91.67 & 99.75 \\
\hline $10 \%$ & 91.45 & 99.72 \\
\hline
\end{tabular}

TABLE 3.2: Radiated sound power levels for different thickness aerofoils at a fixed frequency $\omega=30000 \mathrm{rad} / \mathrm{s}, T I=2.5 \%, \Lambda=7 \mathrm{~mm}$

This observation is consistent with the experimental finding on isolated aerofoils as observed in Chapter 2 of the thesis. The aerofoils geometry effects on turbulence-aerofoil interaction noise are only significant (noticeable) in the high frequency range corresponding to $f t / U>0.3$. For the aerofoil thicknesses to chord ratio $10 \%$, Mach number $M=0.484$, and frequency $\omega=30000 \mathrm{rad} / \mathrm{s}$ presented in the present study on cascades, the non-dimensional frequency $f t / U$ is $\approx 0.18$, which is appreciable lower than the threshold value of 0.3 for which thickness effects become significant. Thus, in the frequency of interest $\leq 3 \mathrm{BPF}$ at approach speeds, influence of aerofoil geometry effects on turbulence-cascade interaction noise is negligible. Even though overall broadband noise is unaffected by changes in aerofoil thickness, the effects are significant on the each individual modes. Clearly, this has significance for tonal noise where, in general, far fewer modes are excited by the periodic mean wake profile. We now demonstrate the effect of aerofoil thickness at each individual mode is due to the combined effects of a variation in blade surface pressure response and differences in propagation of sound in between adjacent blades which might be attributed to reduction in effective pitch with the increase of aerofoil thickness.

We now investigate the effect of thickness on the surface pressure response. Figure 3.18 shows the surface pressure variation for three aerofoil thicknesses. Note that the nose radius is kept constant in this study. The effect of increasing thickness can be seen to have the combined effect of reducing the peak response close to the leading edge and to broaden the response to further downstream. This effect on surface pressure can be attributable to flow acceleration associated with flow separation which is clearly seen in the axial flow contours plotted in figure 3.19 for the $2 \%$ thickness aerofoil. By contrast the mean flow around the leading edge of the $10 \%$ thickness aerofoil is well behaved and fully attached. The change in flow around the leading edge is due to the change in the leading edge wedge angle. Clearly, therefore, the flow and noise is sensitive to the local leading edge geometry which is influenced by both leading edge radius and position of maximum thickness. The second effect of varying aerofoil thickness is to reduce the effective pitch between adjacent blades and hence affect the propagation of cascade waves. 


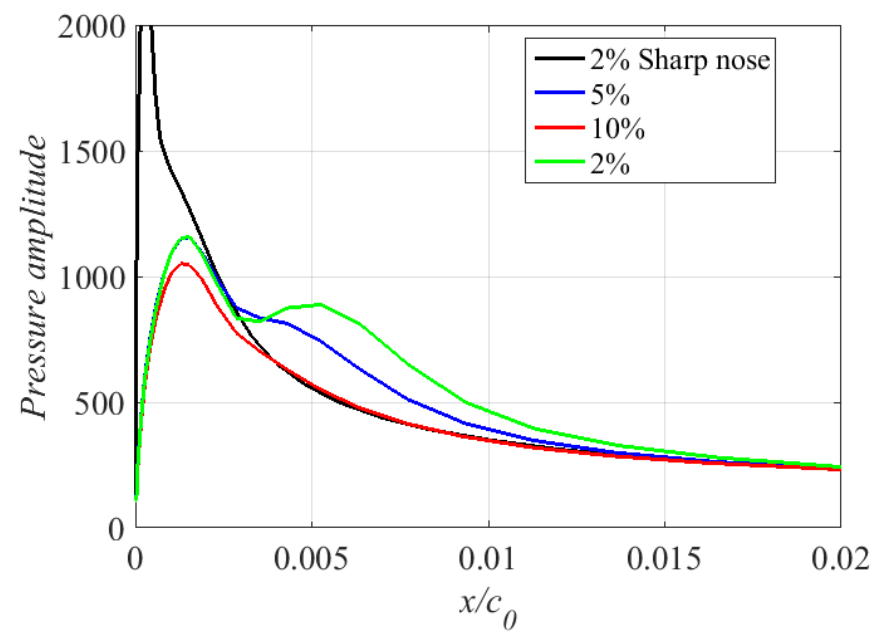

FIGURE 3.18: Unsteady surface pressure on pressure side for various thickness aerofoils at fixed incoming mode, $\mathrm{m}=22$

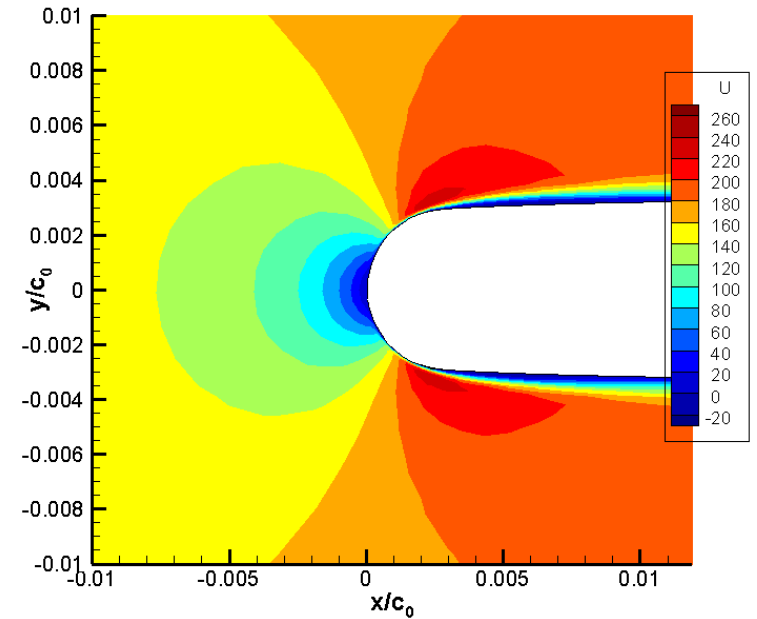

(a) $2 \%$ thickness

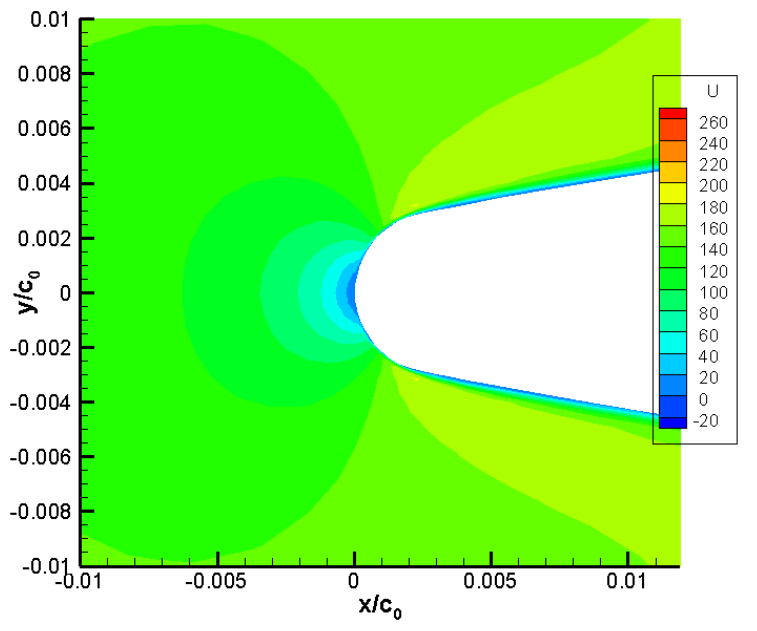

(b) $10 \%$ thickness

FIGURE 3.19: Axial mean velocity around the leading edge of the aerofoil

\section{Effect of aerofoil thickness on staggered aerofoil}

Finally, we investigate the effects of thickness on noise on a staggered aerofoil of stagger

angle $34.19^{\circ}$ with zero camber. Figure 3.20 shows the modal sound power spectrum for aerofoils of varying thickness between 2,5 and $10 \%$. In this non-zero stagger angle case power spectrum is no longer symmetric about $m=B / 2$. It is also weakly affected by variations in blade thickness with differences of less than $0.5 \mathrm{~dB}$. A notable exception is the upstream higher order modes $m \geq 25$ where deviations of up to $5 \mathrm{~dB}$ are observed to thickness variations. The reasons for this need to be explored.

However, as in most other cases discussed above, differences in overall upstream and downstream sound power radiation are negligible, as summarized in Table 3.3. Also tabulated is the power prediction obtained using LINSUB and for the case of zero thickness obtained using HYDRA. Variations in all cases is within $1 \mathrm{~dB}$. 


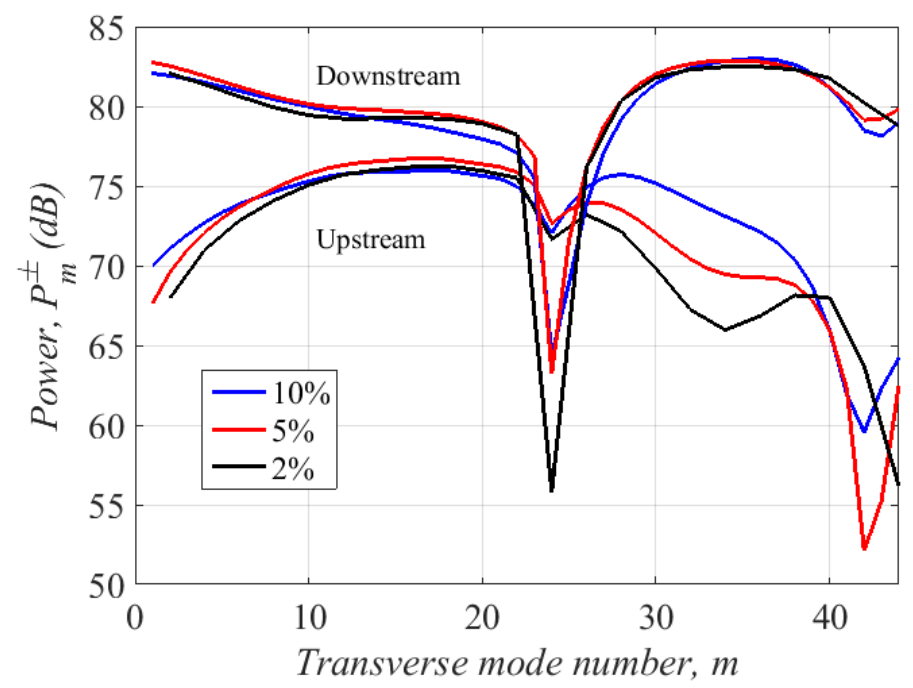

FIGURE 3.20: Influence of thickness on staggered aerofoil at a fixed frequency $\omega=30000 \mathrm{rad} / \mathrm{s}, T I=2.5 \%, \Lambda=7 \mathrm{~mm}$

\begin{tabular}{c|c|c}
$\begin{array}{c}\text { aerofoil thickness } \\
\left(t / c_{0}, \%\right)\end{array}$ & $\begin{array}{c}\text { Upstream Power } \\
(\mathrm{dB})\end{array}$ & $\begin{array}{c}\text { Downstream Power } \\
(\mathrm{dB})\end{array}$ \\
\hline \hline LINSUB & 94.09 & 101.60 \\
\hline Flat plate & 94.48 & 102.18 \\
\hline $2 \%$ & 94.27 & 102.03 \\
\hline $5 \%$ & 95.08 & 102.34 \\
\hline $10 \%$ & 95.43 & 101.95 \\
\hline
\end{tabular}

TABLE 3.3: Radiated sound power levels for different thickness aerofoils with staggered angle $34.19^{\circ}$ at a fixed frequency $\omega=30000 \mathrm{rad} / \mathrm{s}, T I=2.5 \%, \Lambda=$ $7 \mathrm{~mm}$

\section{Effect of aerofoil nose radius}

We now examine the effect of nose radius on broadband noise radiation. In this study aerofoil thickness is held constant at $5 \%$ whilst doubling the nose radius. The mode sound power spectra for the baseline aerofoil and the aerofoil with twice the original nose radius is shown in figure 3.21a. In these two cases the mean flow contours are nearly identical with evidence of mild separation close to the leading edge. The difference in power across all modes is negligible with the greatest effect occurring for the highest order modes which contribute least overall turbulence velocity. The overall change in sound power is therefore negligible.

Similarly, for the case of $2 \%$ aerofoil, we observed an slight increase in mode sound power for the case of sharp nose than compared to baseline nose radius as shown in figure $3.21 \mathrm{~b}$. Here the extreme cases of nose radius are consider to show the influence of nose radius on radiated noise. The total radiated power due to these changes is less than $0.1 \mathrm{~dB}$ which is almost negligible at the highest frequency of interest $(\omega=30000)$ as tabulated in table 3.4. Axial mean flow contours around $2 \%$ sharp aerofoil are plotted in figure $3.22 \mathrm{~b}$. Due to reduction in nose radius, the flow is lesser prone to separation than compared to blunt $2 \%$ aerofoil as observed in figure 3.22a. The purpose of this study is to conclude about the geometric effects such as thickness and nose radius on turbulence-cascade interaction noise is minimum for lower $f t / U$ (where turbulence-cascade noise is dominant). 


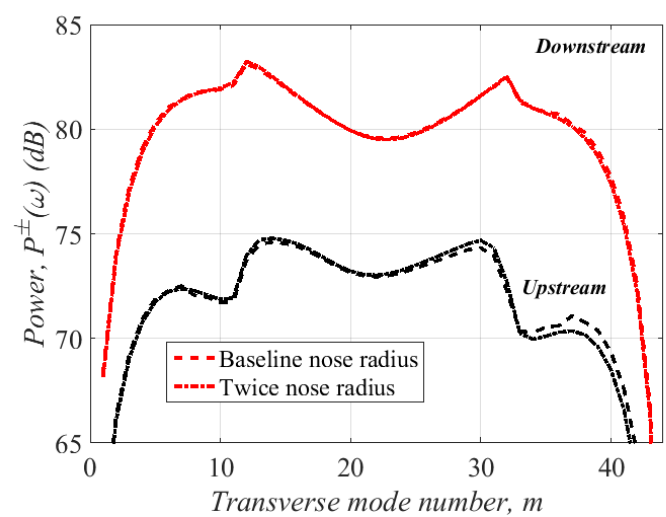

(a) $5 \%$ thickness

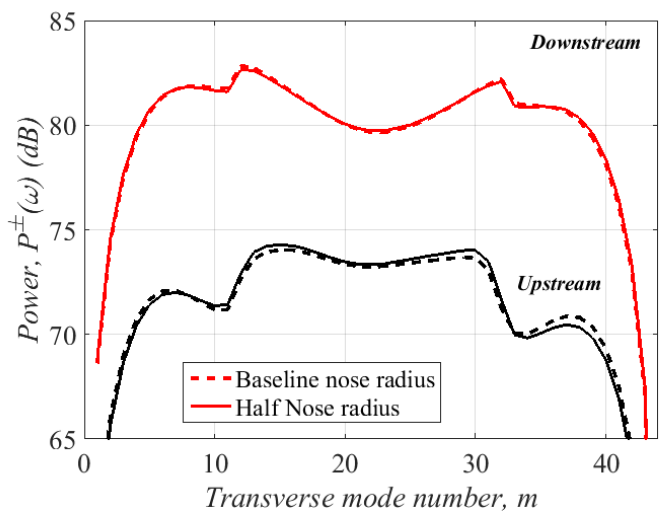

(b) $2 \%$ thickness

FIGURE 3.21: Influence of aerofoil geometry on radiated power for various incoming transverse mode number at a fixed frequency $\omega=30000 \mathrm{rad} / \mathrm{s}$, $T I=2.5 \%, \Lambda=7 \mathrm{~mm}$

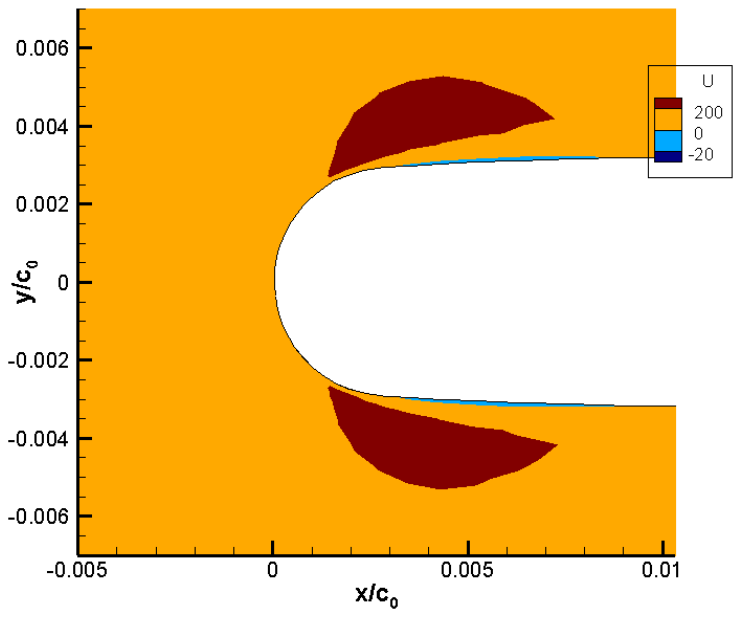

(a) Blunt nose

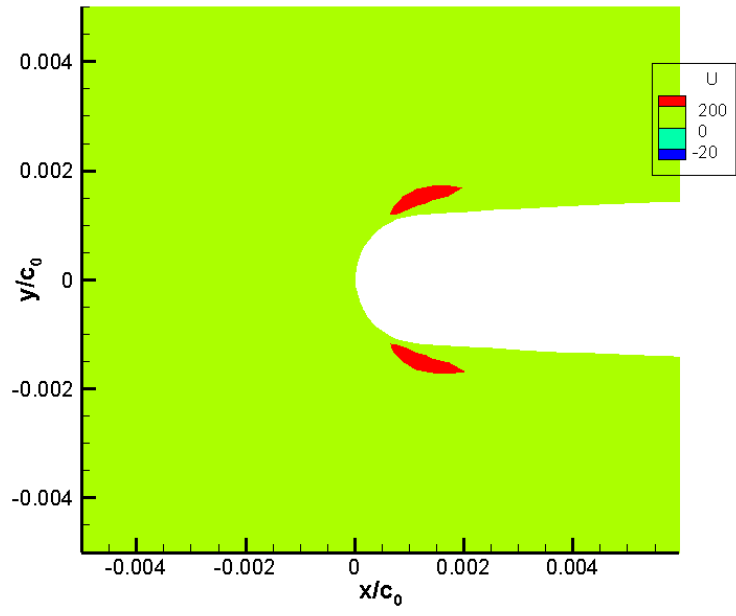

(b) Sharp nose

FiguRE 3.22: Axial mean flow contours around 2\% aerofoil (Zoomed at the aerofoil nose to show flow separation) 


\begin{tabular}{c|c|c|c}
$\begin{array}{c}\text { aerofoil thickness } \\
\left(t / c_{0}, \%\right)\end{array}$ & Nose profile & $\begin{array}{c}\text { Upstream Power } \\
(\mathrm{dB})\end{array}$ & $\begin{array}{c}\text { Downstream Power } \\
(\mathrm{dB})\end{array}$ \\
\hline \hline $2 \%$ & Baseline & 91.46 & 99.91 \\
\hline $2 \%$ & Sharp & 91.36 & 99.90 \\
\hline $5 \%$ & Baseline & 91.78 & 99.94 \\
\hline $5 \%$ & Twice nose radius & 91.84 & 99.91 \\
\hline
\end{tabular}

TABLE 3.4: Influence of aerofoil nose radius on radiated noise

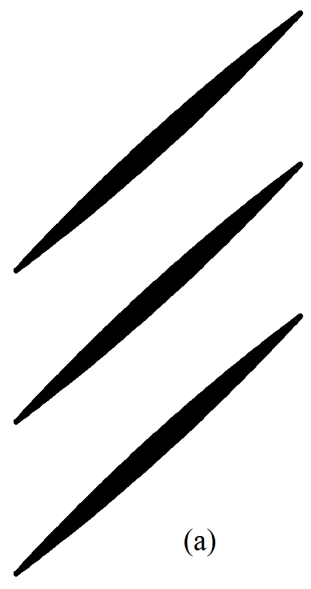

FIGURE 3.23: Schematic of cambered aerofoil geometries (a) No camber (b) Half camber (c) Full camber

\subsubsection{Cambered aerofoils}

The setup of the cambered aerofoil in HYDRA is similar to zero-cambered aerofoil as discussed in section 3.6.1.

\section{Influence of aerofoil camber on interaction noise}

A parametric study has been undertaken aimed at investigating the influence of camber on turbulence-aerofoil interaction noise in a cascade. One motivation for this study is to assess the accuracy of flat plate cascade solutions, such as LINSUB, which of course do not include camber effects. Three different camber profiles having the same leading edge angle are investigated in the current section to preserve the attack angle. In all three cases, the inlet Mach number and flow angle is held constant. The different camber profiles under investigation are shown in figure 3.23. Figure 3.24 shows the upstream and downstream mode power spectra for the three different cambered aerofoils.

Unlike the effects of thickness, camber is observed to have a relatively large effect on the mode power spectrum. One particular difference is observed for mode $m=24$ since it excites an acoustic mode $l=24$ that is now cutoff when there is no camber and the swirl velocity is identical to that at the inlet. However, by removing some components of the swirl by the use of partial or full camber, this modes is now cut-on and cutoff modes now occur for modes $m=28$ and 31 .

This effect is quantified in Table 3.5, which lists the flow speeds and flow angles and cutoff modes for each scattering index $r=0$ and $r=-1$ for the three cases under consideration. 


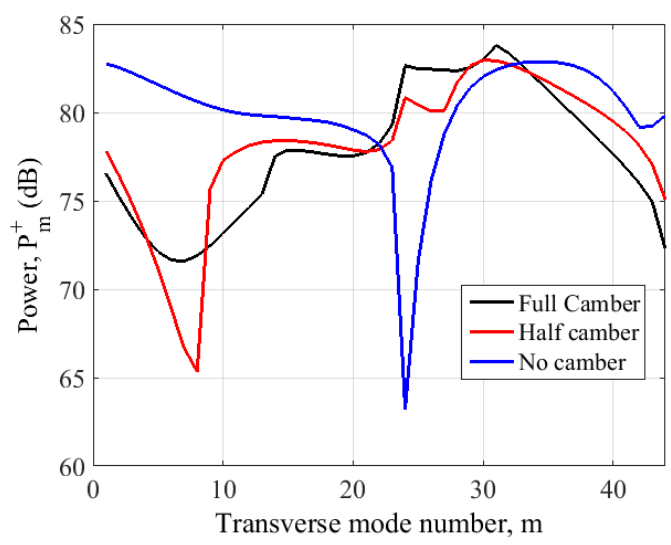

(a) Downstream

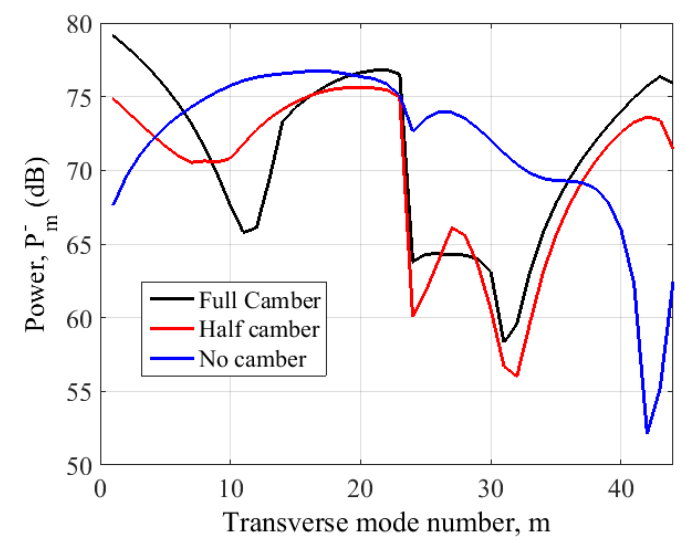

(b) Upstream

FIGURE 3.24: Radiated power versus incoming mode $\mathrm{m}$ for varying camber angle at fixed flow angle of $34.19^{\circ}, \omega=30000 \mathrm{rad} / \mathrm{s}, T I=2.5 \%, \Lambda=7 \mathrm{~mm}$

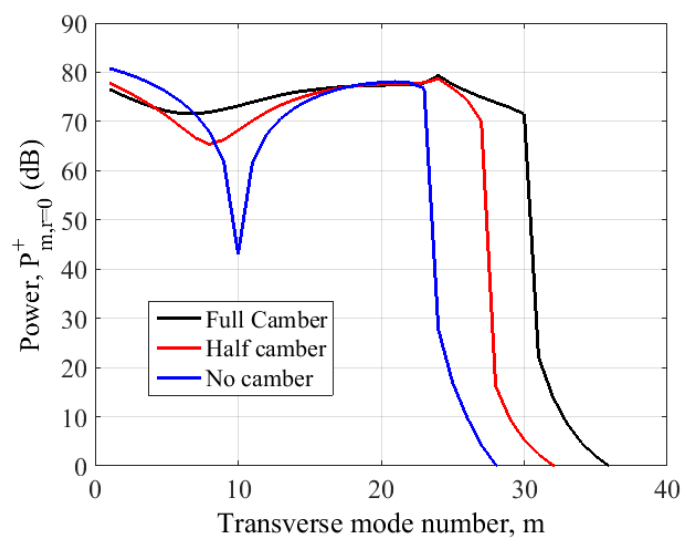

(a) scattering index, $\mathrm{r}=0$

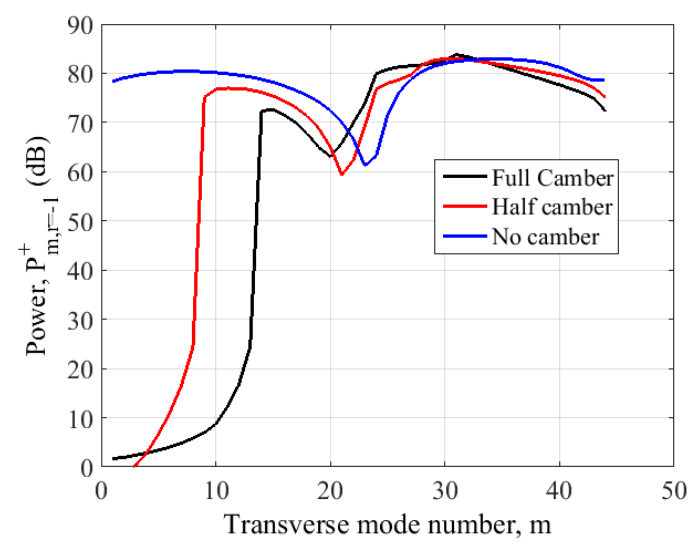

(b) scattering index, $\mathrm{r}=-1$

FIGURE 3.25: Radiated downstream power versus incoming mode $\mathrm{m}$ for varying camber angle at fixed flow angle of $34.19^{\circ}, \omega=30000 \mathrm{rad} / \mathrm{s}, \mathrm{TI}=$ $2.5 \%, \Lambda=7 \mathrm{~mm}$

This cutoff phenomenon associated with the effects of camber are also made clear in the downstream mode power spectral plots in figures 3.25a and $\mathrm{b}$ for $r=0$ and $r=-1$ respectively.

The sound power mode spectral plots shown in figures 3.25a and $\mathrm{b}$ for the downstream sound powers for $r=0$ and $r=-1$ is plotted in figures 3.26a and $\mathrm{b}$ for upstream sound power. In figures 3.26 a only modes $m \leq 24$ excite propagating acoustic modes. There appears a large sensitivity to camber for these range of modes but negligible effect for the cutoff modes $m \geq 24$. In figures 3.26b, however, all modes are cut-on and therefore exhibit a large sensitivity to the effects of camber. The reasons underlying this behaviour are not yet perfectly understood.

The total radiated upstream and downstream power for a fixed frequency of $\omega=30000 \mathrm{rad} / \mathrm{s}$ obtained by summing over all modes is tabulated in Table 3.6. Even though the mode spectra are highly affected by camber the total power varies by less than $1 \mathrm{~dB}$ for the three aerofoil cases. The flat plate solution given by LINSUB, however, cannot incorporate camber effects 


\begin{tabular}{c|c|c|c|c|c|c} 
aerofoil & $\begin{array}{c}\text { Exit Mach } \\
\text { number }(\mathrm{M})\end{array}$ & $\begin{array}{c}\text { Exit Flow } \\
\text { angle }\left(^{\circ}\right)\end{array}$ & $\begin{array}{c}\text { Axial Mach } \\
\text { number } M_{1}\end{array}$ & $\begin{array}{c}\text { Transverse Mach } \\
\text { number } M_{2}\end{array}$ & $\begin{array}{c}\text { Cut-off 'm' } \\
(\mathrm{r}=0)\end{array}$ & $\begin{array}{c}\text { Cut-off 'm' } \\
(\mathrm{r}=-1)\end{array}$ \\
\hline \hline No camber & 0.496 & 35.56 & 0.403 & 0.288 & 24 & 0 \\
\hline Half camber & 0.404 & 17.82 & 0.385 & 0.124 & 28 & 9 \\
\hline Full camber & 0.381 & 0.39 & 0.381 & 0 & 31 & 14 \\
\hline
\end{tabular}

TABLE 3.5: Cut-off modes based on downstream Mach numbers at a fixed frequency $\omega=30000 \mathrm{rad} / \mathrm{s}, T I=2.5 \%, \Lambda=7 \mathrm{~mm}$

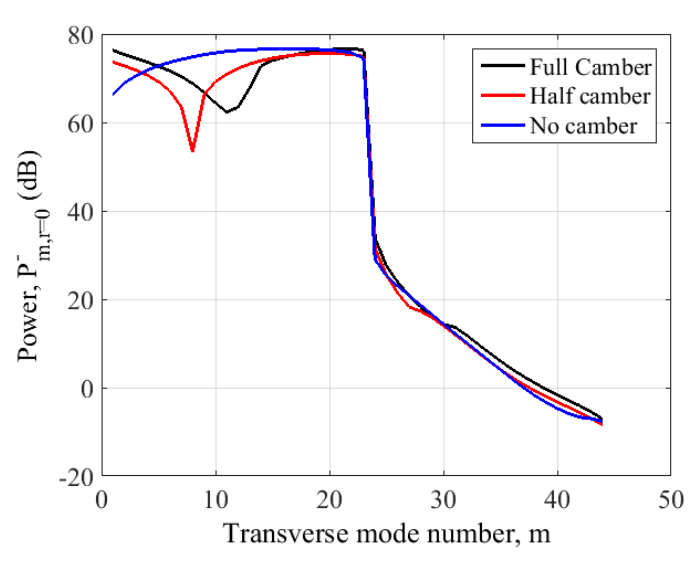

(a) scattering index, $r=0$

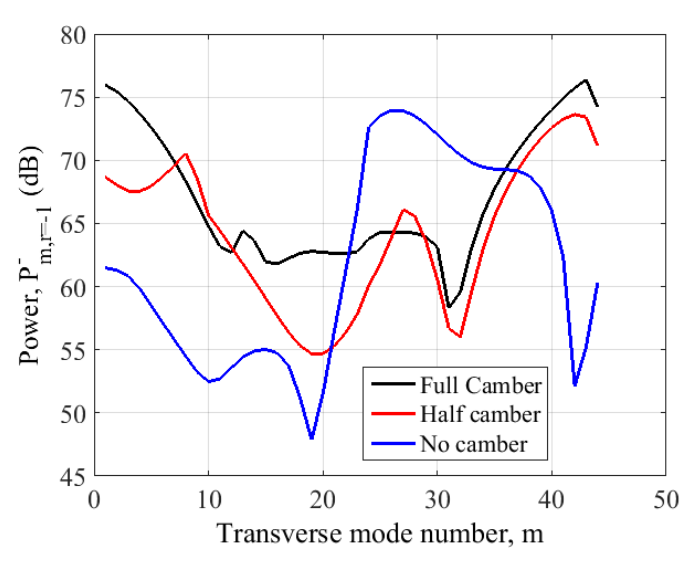

(b) scattering index, $\mathrm{r}=-1$

FIGURE 3.26: Radiated upstream power versus incoming mode $\mathrm{m}$ for varying camber angle at fixed flow angle of $34.19^{\circ}, \omega=30000 \mathrm{rad} / \mathrm{s}, T I=2.5 \%, \Lambda=$ $7 \mathrm{~mm}$

but nevertheless still remains within $1 \mathrm{~dB}$ of the CFD solutions.

\begin{tabular}{c|c|c} 
aerofoil & $\begin{array}{c}\text { Upstream Power } \\
(\mathrm{dB})\end{array}$ & $\begin{array}{c}\text { Downstream Power } \\
(\mathrm{dB})\end{array}$ \\
\hline \hline LINSUB & 94.09 & 101.60 \\
\hline No camber & 95.08 & 102.34 \\
\hline Half camber & 93.65 & 101.02 \\
\hline Full camber & 95.23 & 100.94 \\
\hline
\end{tabular}

TABLE 3.6: Radiated sound power levels for different camber aerofoils at a fixed flow angle of $34.19^{\circ}$ by summing up first 44 modes at a fixed frequency $\omega=30000 \mathrm{rad} / \mathrm{s}, T I=2.5 \%, \Lambda=7 \mathrm{~mm}$

\section{Influence of aerofoil thickness on cambered aerofoil}

In previous sections we observed that the effect of aerofoil thickness and nose radius on cascade interaction noise is negligible for zero-cambered and zero stagger angle aerofoils. We conclude this investigation into the effects of thickness by considering the effects of thickness on realistic OGV-type cascades that include stagger angle and realistic camber.

Three different aerofoils of varying thickness of $2 \%, 5 \%$ and $10 \%$ are now considered for an aerofoil cascade with leading edge angle of $34.19^{\circ}$, zero angle of attack and full camber such that the exists a flow angle of zero, and a mean flow speed of $M=0.484$. A schematic representation of the different thicknesses on cambered aerofoils is shown in figure 3.27. 

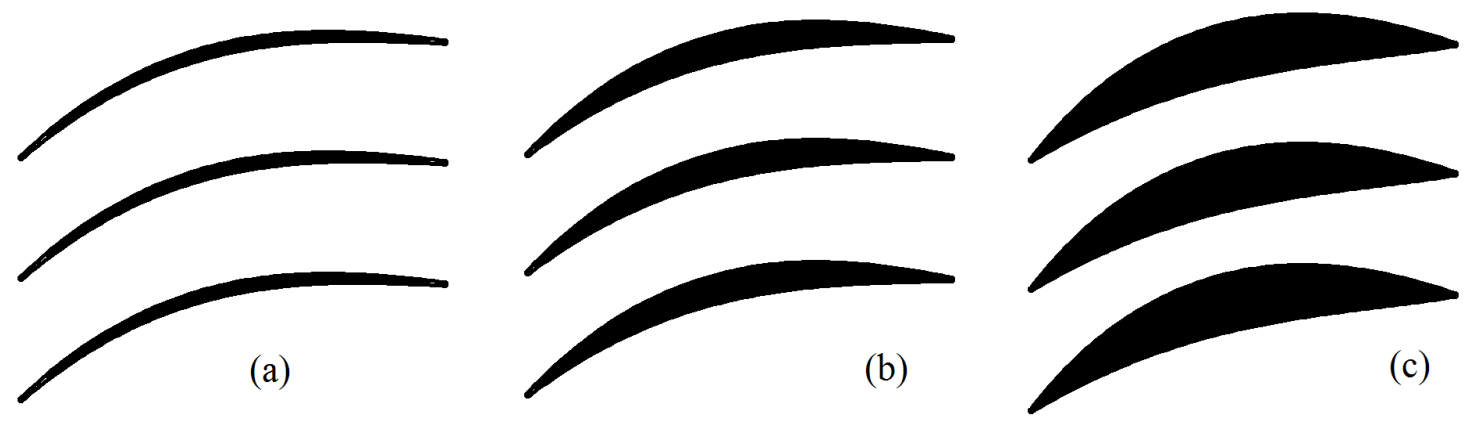

FIGURE 3.27: Schematic of cambered aerofoil geometries (a) 2\% (b) $5 \%$ (c) $10 \%$

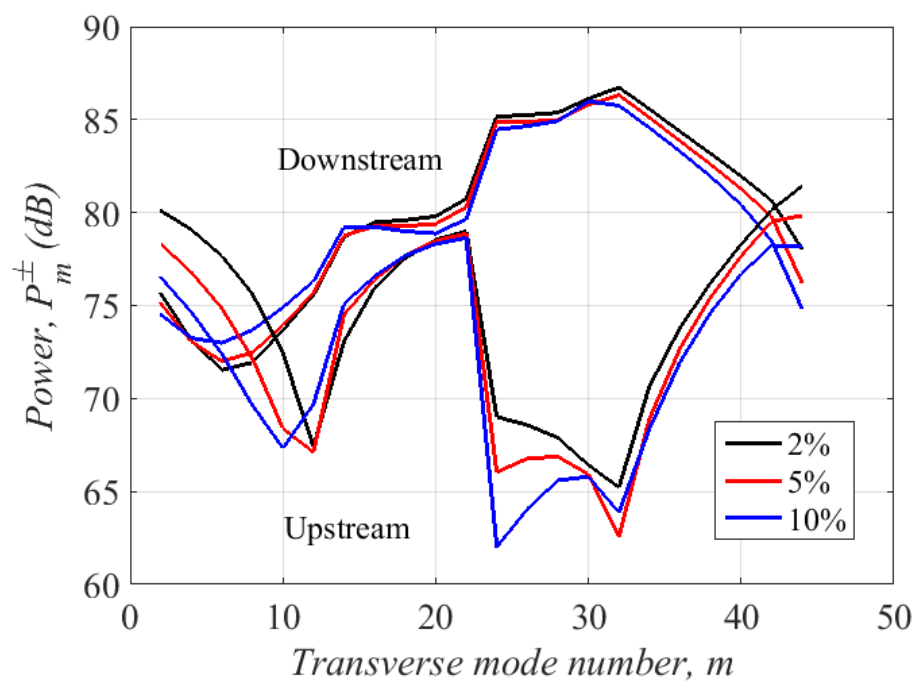

FIGURE 3.28: Radiated power versus incoming mode $\mathrm{m}$ for varying thickness for cambered aerofoils at fixed flow angle of $34.19^{\circ}, \omega=30000 \mathrm{rad} / \mathrm{s}, T I=$

$$
2.5 \%, \Lambda=7 \mathrm{~mm}
$$

The mode sound power spectrum is plotted in figure 3.28 and the total radiated sound power tabulated in Table 3.7. The sound power across all modes and when summed is highest for the thinnest aerofoil of $2 \%$ by about $2 \mathrm{~dB}$. This difference cannot be explained by thickness effects alone, as shown in figure 3.20. The reason for this increase here is due to combined effects of aerofoil stagger and camber. It has been shown in table 3.3 the influence of thickness on stagger aerofoil is within $1 \mathrm{~dB}$. However, the reason for this observation is currently unknown.

\begin{tabular}{c|c|c}
$\begin{array}{c}\text { aerofoil } \\
\left(t / c_{0}, \%\right)\end{array}$ & $\begin{array}{c}\text { Upstream Power } \\
(\mathrm{dB})\end{array}$ & $\begin{array}{c}\text { Downstream Power } \\
(\mathrm{dB})\end{array}$ \\
\hline \hline $2 \%$ & 96.18 & 101.34 \\
\hline $5 \%$ (Roundnose $)$ & 95.05 & 100.95 \\
\hline $10 \%$ & 94.06 & 100.6 \\
\hline
\end{tabular}

TABLE 3.7: Radiated power versus incoming mode $\mathrm{m}$ for varying thickness for cambered aerofoils at fixed flow angle of $34.19^{\circ}, \omega=30000 \mathrm{rad} / \mathrm{s}, \mathrm{TI}=$ $2.5 \%, \Lambda=7 \mathrm{~mm}$ 


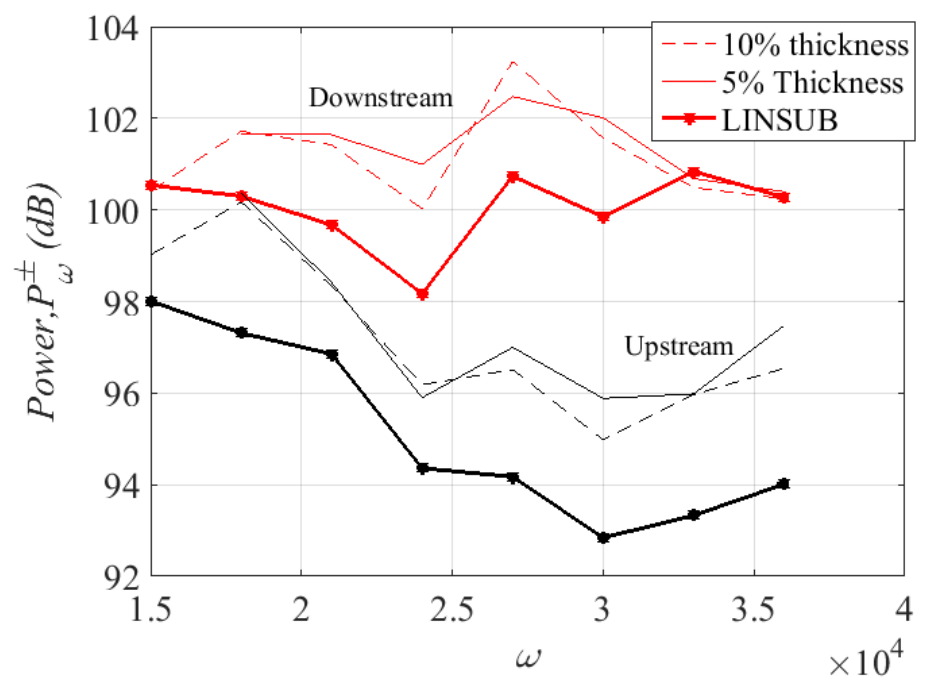

FIGURE 3.29: Overall radiated power across various frequencies at incoming turbulence intensity, $T I=2.5 \%$, and integral length scale $\Lambda=7 \mathrm{~mm}$

\section{Broadband comparison for cambered aerofoil}

The overall sound power spectrum versus frequency at 12 discrete frequencies in the frequency range $\omega=5000$ to 35,000 (roughly $0.5 \mathrm{BPF}$ and 3BPF) for the case investigated above for the $5 \%$ and $10 \%$ thickness cases is plotted in Figure 3.29. Differences across all frequencies are approximately less than $1 \mathrm{~dB}$ for these relatively thick aerofoils but appears to slightly increase as frequency increases. It therefore appears that the effects of thickness found on isolated aerofoils also applies to realistic aerofoils in a cascade with $f t / U$ being the main controlling parameter. Figure 3.29 also shows the LINSUB comparison with the staggered and cambered aerofoil and the difference of $1.5 \mathrm{~dB}$ is observed. The reason for the difference is attributed to camber effects as the LINSUB solution doesn't account the aerofoil geometry effects.

\section{Influence of Flow incidence on interaction noise}

Previous sections have suggested that the flow behaviour in the passage is a relatively important factor in determining broadband noise radiation. Especially, the effect of stagger and camber plays a significant role in determining the radiated power. With the change of flow incidence, the similar changes in the flow behaviour in the passage is observed. We therefore conclude this chapter with a study into the effects of flow incidence angle on turbulence-cascade interaction noise. The noise at a total of 6 different flow incidence angles was computed on the same cambered aerofoil as above with $5 \%$ thickness. The upstream and downstream sound power is plotted in figure 3.30. A shift in the mode power spectra is observed which is fully consistent with the difference in the ratio of axial to swirl velocities associated with the different incidence angles. The effects of incidence angles is therefore two-fold. One is to alter the degree of flow separation close to the leading edge; the other is to alter the modal cutoff frequencies in the duct, both upstream and downstream of the cascade.

Finally, the total radiated power at $\omega=30000 \mathrm{rad} / \mathrm{s}$, frequency is plotted in figure $3.30 \mathrm{~b}$ against flow incidence angles for incoming turbulence with $T I=2.5 \%$ and $\Lambda=7 \mathrm{~mm}$. 


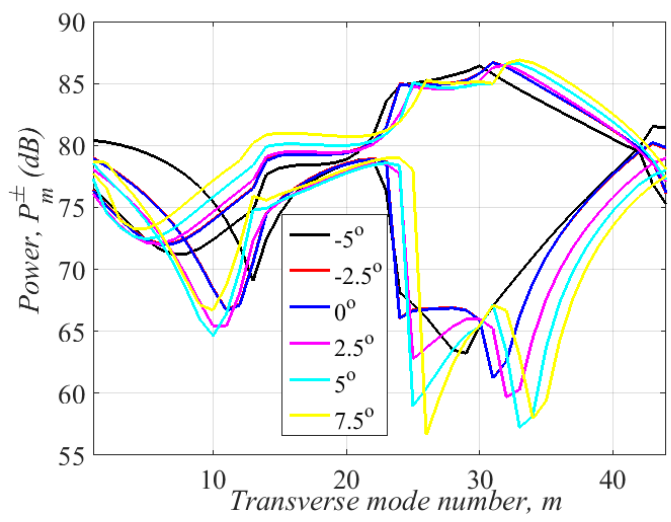

(a) Radiated sound power level, $P_{m}^{ \pm}$

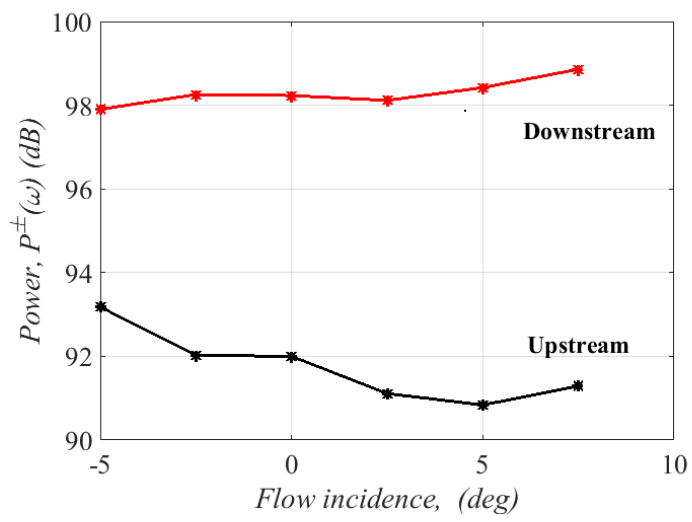

(b) Total radiated sound power level, $P^{ \pm}(\omega)$

FIGURE 3.30: Influence of flow incidence on radiated noise at $\omega=$ $30000 \mathrm{rad} / \mathrm{s}, T I=2.5 \%, \Lambda=7 \mathrm{~mm}$

Downstream sound power remains largely unaffected by the incidence angle while the upstream sound power appears to reduce with increasing incidence angles. This effect is linked to the behaviour of the flow region around the leading edge and in the passage. More work is needed to understanding this phenomenon more clearly. The idea of presenting these results is to show the capabilities of the present approach to predict the upstream and downstream radiated power for slightly separated flows.

\subsection{Conclusions}

This chapter presents a numerical approach to the prediction of the broadband noise due to the interaction between 2D cascades with 2D turbulent flow. It is based on a Fourier synthesis of the turbulence. The aerofoil response due to each mode is considered in turn. The approach has been validated against the well-known flat plate solution, LINSUB. The numerical approach was then used to show that the periodicity property in the blade response for the flat plate cascade was also sufficiently accurate to be used to allow the modal powers for a realistic aerofoil cascade to be summed infinity by only computing the blade response for modes between 0 and $B$.

The effects of viscosity on the mean flow behaviour was found to be important in controlling flow separation and boundary layers close to the leading edge, which in turn has been shown to affect broadband noise radiation $(\approx 0.3 \mathrm{~dB}$ on aerofoil).

The general approach was used to study the effects of aerofoil thickness, stagger angle, camber and flow incidence. The general conclusion is that these factors have a potentially large effect on individual modes. However, when summed to compute overall broadband noise, the differences were generally negligible.

It is shown that flat plates approximation is good assumption to predict the radiated power within $1.5 \mathrm{~dB}$. The major drawback of the flat plate model LINSUB is that it doesn't account the aerofoil camber effects which are slightly significant.

This chapter has shown geometry effects by themselves for realistic mean flow velocities and frequencies do not significantly effect broadband noise, which is consistent with the observation made for isolated aerofoils that $f t / U$ is the dominant controlling factor. For 
full-scale OGV's at about 3BPF, $f t / U \leq 0.1$ and therefore geometry effects are predicted to be insignificant. However, some combination of blade geometry and incidence angles have been shown to provide levels of flow separation that can have a detrimental effects on broadband noise. More work is needed to examine these effects further and extend the approach to three dimensions. It was shown in literature, blade swipe effects on the broadband interaction noise is minimum.

Hence, the way forward is usage of innovative leading edge geometries to reduce turbulencecascade interaction noise. A preliminary experimental test campaign has been performed on realistic OGV geometries to demonstrate the broadband noise reductions with leading edge serrations (See Appendix D.1). 


\title{
Chapter 4
}

\section{Turbulence-aerofoil interaction noise reduction using sinusoidal serrations}

\author{
Contents

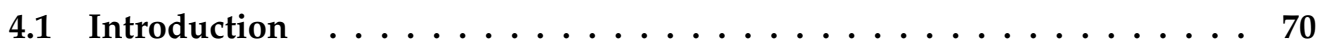

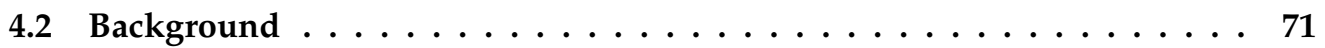

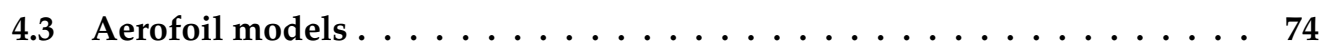

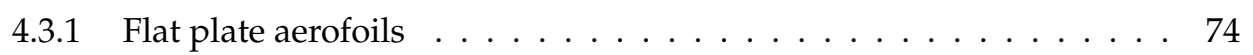 \\ 4.3 .2 3D Aerofoil models . . . . . . . . . . . . . . . . . . 75 \\ 4.4 Acoustic Performance $\ldots \ldots \ldots \ldots \ldots \ldots \ldots$ \\ 4.4.1 Overall acoustic performance on flat-plates . . . . . . . . . . 76 \\ 4.4.2 Sound power reductions versus non-dimensional frequency $f h / U$. 76 \\ 4.4 .3 Optimal serration wavelength $\ldots \ldots \ldots \ldots \ldots 77$ \\ 4.4 .4 Interpretation $\ldots \ldots \ldots \ldots \ldots$ \\ 4.4 .5 Noise reduction contours . . . . . . . . . . . . . . . . 86 \\ 4.4.6 Influence of self-noise on aerofoils on 3D aerofoils . . . . . . . 88 \\ 4.4.7 Influence of leading edge serrations on trailing edge self-noise . . . . 88 \\ 4.4.8 Validity of optimal serration angles for 3D aerofoils . . . . . . . . 90 \\ 4.4 .9 Comparison with serrated flat plate . . . . . . . . . . . . . 91 \\ 4.4 .10 Effect of angle of attack . . . . . . . . . . . . . . 92 \\ 4.5 Aerodynamic Measurement . . . . . . . . . . . . . . . 93 \\ 4.6 Flow measurement . . . . . . . . . . . . . . . . . . 94 \\ 4.6 .1 Mean flow . . . . . . . . . . . . . . . . . . . . . 94 \\ 4.6.2 Effect on leading edge serrations on boundary layer development . . 95 \\ 4.6.3 Wake characteristics . . . . . . . . . . . . . . . . . . 97

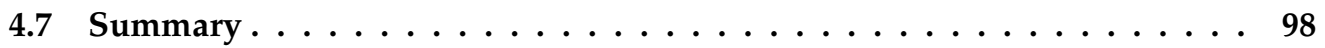

This chapter presents the results of a detailed experimental investigation into the effectiveness of sinusoidal leading edge serrations on aerofoils for the reduction of noise generated by the interaction with turbulent flow. A detailed parametric study is performed to investigate the sensitivity of the noise reductions to the serration parameters, in particular the serration amplitude and wavelength. The study is primarily performed on flat plates in an idealized turbulent flow, which we demonstrate captures the same behaviour as when identical serrations are introduced onto 3D aerofoils. The influence on the noise reduction of the turbulence integral length-scale is also studied. An optimum serration wavelength is identified whereby maximum noise reductions are obtained, corresponding to when the transverse 
integral length-scale is roughly one-forth the serration wavelength. One of the most important findings of this chapter is that, at the optimum serration wavelength, the sound power radiation from the serrated aerofoil varies inversely proportional to the Strouhal number $S t_{h}=f h / U$, where $f, h$ and $U$ are frequency, serration amplitude and flow speed, respectively. A simple model is proposed to explain this behaviour. Noise reductions are observed to generally increase with increasing frequency until the frequency at which aerofoil selfnoise dominates the interaction noise. Leading edge serrations are also shown to reduce trailing edge self-noise. The mechanism for this phenomenon is explored through PIV measurements. Finally, the lift and drag of the serrated aerofoil were obtained through direct measurement and compared against the straight edge baseline aerofoil. It is shown that aerodynamic performance is not substantially degraded by the introduction of the leading edge serrations on the aerofoil.

\subsection{Introduction}

Modern turbofan engines have increasingly high bypass ratios. Fan broadband noise has therefore become a dominant noise source, particularly at approach conditions. One of the major noise sources arises from the interaction between rotor wake turbulence and the leading edge of the downstream Outlet Guide Vanes (OGV's). Recent flightpath 2050 targets have been set aimed at reducing noise emissions by $65 \%$ by 2050 . Wind turbines are another important environmental noise source where the interaction of turbulence with the aerofoil leading edge is believed to the dominant noise source at low frequencies in which large-scale atmospheric turbulence interacts with the rotating blades.

The effects of aerofoil geometry on turbulence-aerofoil interaction noise has been studied extensively (Gershfeld, 2004; Roger, 2010; Moriarty et al., 2005; Lysak et al., 2013; Gill et al., 2013; Devenport et al., 2010; Evers and Peake, 2002; Chaitanya et al., 2015a). It has been demonstrated by (Narayanan et al., 2014; Haeri et al., 2014; Narayanan et al., 2015; Chaitanya et al., 2015b; Kim et al., 2016) and others that introducing leading edge serrations can be an effective method of reducing far field noise. This previous work has demonstrated the sensitivity of noise reductions to the serration geometry. However, this early work has focused mainly on flat plates where the entire interaction process is confined to the plane of the plate. Three dimensional flow effects were therefore absent from these studies. The present chapter aims to identify the optimum serration geometry and apply it to aerofoils. A comprehensive study into the effectiveness of sinusoidal leading edge serrations is presented for reducing aerofoil interaction noise that includes an investigation into their effect on aerodynamic performance. Acoustic measurements are made alongside aerodynamic measurements to provide a detailed assessment of their potential effectiveness in reducing the noise from an aerofoil of $10 \%$ thickness and 1.2 camber, which is expressed as designed lift coefficient. This chapter also presents a preliminary investigation into the use of Leading Edge (LE) serrations for the reduction of trailing edge self-noise. Detailed noise measurements are made on both flat plates and 3D aerofoils. Similar noise reduction characteristics are observed in both cases suggesting that the flat plate experiments capture the essential physical noise reduction mechanisms (Narayanan et al., 2014; Haeri et al., 2014).

The objectives of this chapter are as follows:

1. To examine the sensitivity of noise reductions to variations in serration parameters (amplitude and wavelength) and turbulence integral length-scale on flat plates. 
2. To apply the above findings to inform the design of effective leading edge serrations on $3 \mathrm{D}$ aerofoils.

3. To investigate the effect of leading edge serrations on trailing-edge self noise.

4. To quantify the relative contributions of interaction noise and self-noise to the overall radiated noise spectrum with and without leading edge serrations.

5. To provide a simple model to predict the observed frequency dependence of the noise reduction spectra.

6. To visualize the flow around the peak and root regions of the leading edge serration to assess their effect on the steady aerodynamic behavior.

7. To quantify the aerodynamic performance of serrated aerofoils at low angles of attack.

\subsection{Background}

Leading edge serrations can be found on owl wings and whale flippers to reduce noise and enhance hydro and aerodynamic performance. It has long been established that introducing LE serrations on aerofoils can improve their aerodynamic performance at post-stall conditions (Skillen et al., 2014; Zhang et al., 2013; Hansen et al., 2011; Yoon et al., 2011; Johari et al., 2007). Collins (1981) has observed that the presence of leading edge serrations on wings can improve the low-speed lift and stall performance of aircraft during take-off and landing. Bachmann et al. (2007) showed that the barn owl exhibits 'silent' flight due to serrations at the leading edge of the wing and the fringes at the edges of each feather. They proposed that the topographies and mechanisms underlying this silent flight might eventually be employed for aerodynamic purposes thus resulting in new wing designs in modern aircraft. They showed that the owl is quieter than the pigeon due to the presence of serrations at its leading edge and the fringes at the edges of each quill.

Soderman (1972) was one of the first to investigate the aerodynamic effects of LE serrations on an aerofoil in a closed wind tunnel. He observed that, at a flow speed Mach number of 0.13 , by placing small serrations on the aerofoil leading edge, vortices were generated which could enhance the maximum lift at high angles of attack. It was also observed that the presence of small amplitude serrations on the aerofoil does not increase the drag at smaller angles of attack and reduces it at larger angles. Visualization of the flow showed that the serrated edges introduce vortices which energizes the boundary layer thereby delaying leading edge flow separation at higher angles of attack.

Hersh et al. (1974) have demonstrated the effectiveness of LE serrations in reducing the narrow band vortex shedding noise radiated from stationary and rotating aerofoils. Noise reductions of between 4 and $8 \mathrm{~dB}$ were observed at the peak shedding frequency, which they attributed to the formation of vortices breaking up the periodic structure of the wake.

A significant amount of work has been undertaken experimentally and numerically aimed at assessing the effectiveness of leading edge serrations on delaying stall. Miklosovic et al. (2004) conducted the first windtunnel tests using idealized models of humpback whale flippers at mean chord Reynolds numbers in the range of $5.05 \times 10^{5}-5.20 \times 10^{5}$ and attack angles in the range of $-2-20 \mathrm{deg}$. They demonstrated experimentally that the angle of attack at which stall occurred was significantly delayed when leading edge serrations were introduced onto a model whale flipper geometry. Miklosovic et al. (2007) performed the aerodynamic evaluation on a full-scale humpback whale flipper geometry to simulate the 
effects of LE tubercles. They demonstrated experimentally that serrations on the aerofoil leading edge produced vortices from the serration peaks which significantly altered the performance of the serrated aerofoil. The significance of these vortices in affecting aerodynamic behaviour was also observed by Stanway (2008) in PIV measurements of the flow in the vicinity of a serrated leading edge. A number of researchers (Fish and Lauder, 2006; Fish et al., 2011; Zhang et al., 2013; Van Nierop et al., 2008) have investigated the mechanism by which stall can be delayed by the introduction of serrations at the aerofoil leading edge. They observed that the LE serrations generate streamwise vortices, which were attributed to greatly enhanced momentum transfer. This results in a significant reduction of flow separation and therefore improvement in the aerofoil aerodynamics within the wide range of post-stall angles of attack. More recently, Skillen et al. (2014) have also explored the mechanism involved in the reduction of the separation region at high angles of attack by the use of serrated aerofoils. They showed that the serrated leading edge introduces a strong span-wise pressure gradient which results in the formation of secondary flows (additional stream-wise flow along the serration edge). This secondary flow transports the near-wall fluid which is replaced by the high-momentum fluid available in the flow. The boundary layer is then re-energized which might be a reason for the delay in flow separation for serrated aerofoils.

Recently, Rostamzadeh et al. (2013) proposed a design of leading edge comprising out-ofplane sinusoidal modulations with the objective of improving aerodynamic performance. They compared the aerodynamic characteristics of aerofoils with tubercles (modulation in the chord plane) with predictions obtained from Prandtls nonlinear lifting-line theory. They demonstrated that both in-plane and out of plane leading edge modulations have similar aerodynamic lift and drag characteristics. The wavy serrated aerofoil with the highest peakto-root amplitude and smallest wavelength was found to have the most favorable post-stall behavior. They also showed, using CFD, that the root of the serrated aerofoils were subjected to adverse pressure gradients resulting in flow separation.

Favier et al. (2012) performed a DNS study on serrated geometries for low Reynolds number flow. They observed a 35\% reduction in drag and a significant reduction in lift compared with the baseline (straight leading edge) aerofoil. Hansen et al. (2011) measured the lift and drag of several serrated geometries. They observed that, for the aerofoil of maximum thickness at 50\% chord, the effect on lift in the pre-stall regime was negligible but beneficial in the post-stall regime. For the NACA0021 aerofoil, where the maximum thickness is at $30 \%$ chord, the lift performance in the post-stall regime increased but was degraded in the pre-stall regime. It was also observed that optimizing the serration amplitude and wavelength can increase the lift performance in both pre-stall and post-stall regimes. Skillen et al. (2014) has noted discrepancies between the measured and predicted lift on serrated aerofoils, which he attributed to uncertainties in reproducing wind-tunnel experimental conditions.

Chong et al. (2015) has measured the lift and drag of a serrated aerofoil and found that increasing the serration wavelength tended to improve performance at angles of attack close to stall. However, the lift coefficient in the pre-stall region was observed to be lower compared to the baseline (straight edge) aerofoil. The explanation for the increased stall angle compared to the straight edge aerofoil was investigated using oil-visualization. In the case of a straight leading edge, boundary layer separation is apparent whereas LE serrations produce counter-rotating vortices causing the boundary layer separation to be suppressed.

Whilst the use of leading edge serrations have been investigated extensively for improving aerodynamic performance, comparatively little work has been undertaken aimed at its aeroacoustics performance. 
Roger et al. (2013) has formulated an analytic model for the sound generation due to a turbulent flow interacting with a flat plate serrated leading edge of infinite chord. His model treats the serrations as a continuously varying leading-edge sweep whose unsteady aerodynamic response is predicted by splitting the airfoil into strips of small spanwise extent and assimilating the local curved edge to its tangent to make each strip a slice of a swept airfoil, whose response functions are known. The model makes explicit the relative significance of super-critical and sub-critical gust components to the overall far field noise radiation. Lau et al. (2013) has investigated numerically the effects of serrated leading edges on the noise due to a single harmonic vortical gust. This work has demonstrated that one of the key factors in determining the level of noise reduction is the ratio between the leading edge amplitude to the hydrodynamic wavelength. This finding is supported by the experimental work presented in the present chapter for a turbulent in-flow but only at the optimum serration angle which is related to the turbulence integral length scale. Lau et al. (2013) found from numerical simulations that significant noise reductions were achieved when the ratio between the leading edge amplitude to the gust wavelength exceeds about 0.3 . In their paper they attribute the reductions in noise to a more rapid phase variation of pressure fluctuations along the serrated LE compared to the straight leading edge. This explanation of the noise reduction mechanism is consistent with the strip model proposed by Roger et al. (2013).

Clair et al. (2013) has presented a numerical and experimental investigation into the effect of sinusoidal leading edge serrations for the reduction of turbulence-aerofoil interaction noise. Reductions in sound power level over a wide frequency range of between 3 and $4 \mathrm{~dB}$ were both measured and predicted for a NACA65 aerofoil with $0.15 \mathrm{~m}$ chord over a range of flow speeds between 20 to $80 \mathrm{~m} / \mathrm{s}$. The reason for these modest noise reductions compared to the much larger noise reductions presented in (Narayanan et al., 2015) is due to the relatively short serration amplitudes investigated. In Clair et al. (2013) noise reductions at high frequencies (between about 3 and $4 \mathrm{kHz}$ ) were predicted to be greater than that measured, which were attributed to the span-wise gusts contribution being neglected in the computations.

Through numerical simulations based on the compressible three-dimensional Euler equations and a synthetic eddy method for the turbulence generation, Kim et al. (2016) investigated the noise reduction mechanisms of sinusoidal leading edge serrated aerofoils. Kim et al. (2016) found that the surface pressure fluctuations along the leading edge exhibit a source cut-off effect due to oblique edge which results in reduced radiated sound power levels. He also demonstrated destructive interference effect between the peak and hill region is one of the reason for noise reductions.

Lyu et al. (2016) has developed a mathematical model to predict the sound radiated by serrated leading edge geometries. The model is based on an iterative form of the Amiet approach solved using the Schwartzschild technique. The solution most likely converges to the exact solution for a single component of gust turbulence. However, in practice only a few terms were needed to obtained good convergence of the solution. The response to a turbulent flow is synthesised by the summing the response incoherently due to each oblique gust component. The predictions are in close agreement with the experimental data. In this paper, Lyu et al. (2016) attribute the noise reduction mechanism to destructive interference of the scattered surface pressure induced by the presence of serrations.

(Narayanan et al., 2015) have recently undertaken a preliminary parametric study to quantify experimentally the sensitivity of the reductions in radiated noise to variations in the serration amplitude and wavelength. This chapter has identified the minimum frequency $f_{3 d B}$, above which significant noise reductions are achieved $(>3 d B)$. For all the serration 
geometries investigated, $f_{3 d B}$ was observed to closely follow the relationship $f_{3 d B}=\alpha U / 2 h$, where $h$ is the serration amplitude, $U$ is the flow velocity, and $\alpha$ is a constant varying between 0.4 and 0.6. This relation is consistent with the simulations of Clair et al. (2013), who found that the frequency range where noise reductions are observed to increase as the mean flow speed $U$ is reduced. Most of this work in Narayanan et al. (2015) was performed on flat plates with only a limited comparison being presented with serrations on 3D aerofoils.

Chaitanya et al. (2015b) showed the possible existence of an optimum serration inclination angle $\theta_{o}$ at which maximum noise reductions occur. This angle was found to be dependent on the integral length scale of the incoming turbulence. The evidence of an optimal serration angle $\theta_{o}$ may help to explain the conflicting findings of Narayanan et al. (2015) and Hansen et al. (2011), regarding the influence of LE wavelength on noise reductions. It is likely that in the cases investigated by Narayanan et al. (2015) the leading edge profiles represent serration angles greater than the optimum angle, resulting in sub-optimal noise reductions. In the case of Hansen et al. (2011) the opposite is true and the inclination angles investigated were smaller than the optimum angle identified in Chaitanya et al. (2015b).

The present study explores this finding in greater detail. Here we show that the optimum serration wavelength, rather than angle, provides a more fundamental interpretation of the experimental noise data. More recent work by Kim et al. (2016) has investigated numerically the possible noise reduction mechanism due to leading edge serrations. These noise reduction mechanism identified are inline with our current experimental results. In the present chapter the preliminary findings outlined above are investigated in greater detail. The sound power reduction spectra are observed to collapse when plotted against Strouhal number, $S t_{h}$, where $S t_{h}=f h / U$ and $f, h$ and $U$ are frequency, serration amplitude and flow speed, respectively. This finding strongly suggests that, at the optimum serration wavelength, noise reductions are determined solely by the ratio of the serration amplitude $h$ to hydrodynamic wavelength $U / f$. Moreover, at the optimum wavelength, sound power reductions are observed to follow an inverse Strouhal dependence $\propto 1 / S t_{h}$. A very simple model is developed in this chapter aimed at interpreting this Strouhal dependence.

\subsection{Aerofoil models}

\subsubsection{Flat plate aerofoils}

A parametric experimental study was undertaken to investigate the effect on radiated noise due to variations in serration amplitude and wavelength on flat plates based on the assumption that the flat plate serrations capture the same noise reduction mechanisms as that on 3D aerofoils. The optimal serration geometry identified from this flat plate study was used to investigate a limited number of 3D aerofoils.

The serrated flat plate is constructed from two metallic plates of $1 \mathrm{~mm}$ thickness riveted together. The flat plate serrations made from acrylic plate of $2 \mathrm{~mm}$ thickness are inserted in between the two $1 \mathrm{~mm}$ plates. The two steps caused by serration inserts are 'ground down' to smooth the step. The trailing edge of the plate is sharpened to prevent vortex shedding noise, although the leading edge was left blunt i.e. was not sharpened like trailing edge for consistency across all serrations investigated. The dimensions of the flat plate are $15 \mathrm{~cm}$ mean chord and $45 \mathrm{~cm}$ span. A schematic of the serrated sinusoidal geometry is shown in figure 4.1(a) located downstream of a single turbulent eddy whose size is one forth the serration wavelength, which we will later show corresponds to the optimum serration angle for 


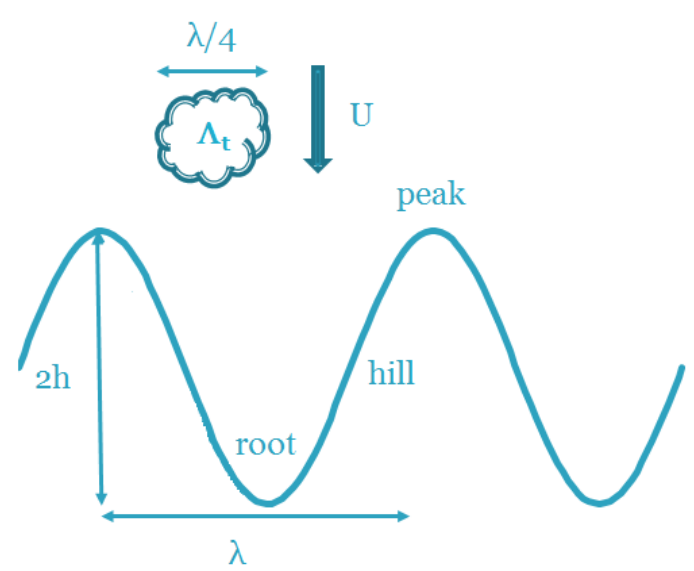

(a) Schematic representation of serrated leading edge

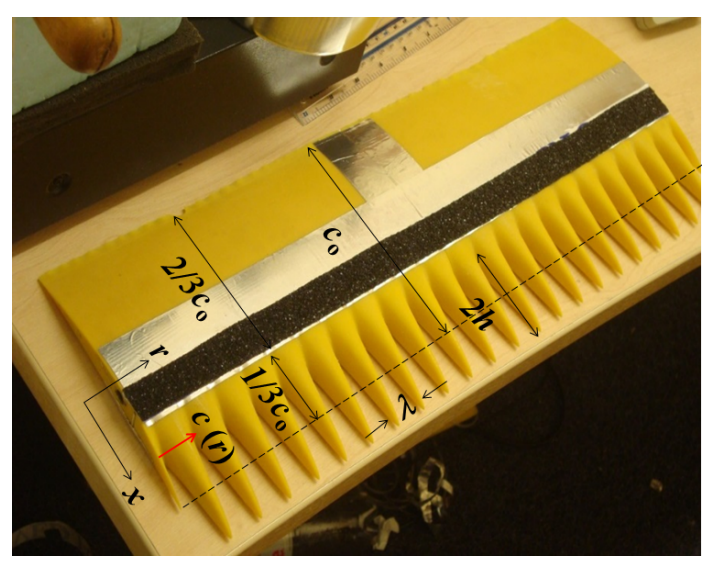

(b) A photograph of the LE serrated aerofoil showing all the parameters

FIGURE 4.1: Leading edge geometry where Serrated wavelength, $\lambda$; Serrated amplitude, $2 h$; Flow speed, $U$; Transverse integral length scale of incoming turbulence, $\Lambda_{t}$; mean chord length, $c_{0}$

maximum noise reduction. In the present study a total of 50 flat plate serrations were investigated. A systematic variation of serration wavelength $\lambda / \Lambda_{t}$ from 0.8 to 100 was investigated comprising five different serrations amplitudes $\left(h / c_{0}\right)$ of $0.033,0.67,0.1,0.133 \& 0.167$.

\subsubsection{D Aerofoil models}

The results from the flat plate study presented in section 4.4 .3 below were used to define five 3D serrated leading edge aerofoil geometries on the NACA-65(12)10 aerofoil. Serration wavelength ranged between 2.67-8, chosen to be close to the optimum wavelength $\lambda_{o}$ of $\approx 4$ (discussed below in Section 4.4.3). These were fabricated using a 3D printer from durable photo-polymer that has high quality surface finish. Three serration amplitudes $\left(h / c_{0}\right)$ of $0.067,0.1,0.167$, with constant serration wavelength $\left(\lambda / \Lambda_{t}\right)$ of 2.67 were investigated. Two of the aerofoils were chosen to have constant amplitudes $\left(h / c_{0}\right)$ of 0.167 with differing serration wavelengths $\left(\lambda / \Lambda_{t}\right)$ of $5.33,8$.

A photograph of a typical serrated aerofoil is shown in figure 4.1(b), with the serration parameters, wavelength $\lambda$, total peak-root distance $2 h$, mean chord $c_{0}$, defined. The serrated leading edge profiles are such that if $y(X)=f(X)$ defines the variation of height above the origin for the NACA-65(12)10 aerofoil profile, where $X=0$ represents the trailing edge and $X=1$ the leading edge, the profile $y(X, r)$ at any span-wise position $r$ along the aerofoil is given by,

$$
y(X, r)= \begin{cases}f\left(x / c_{0}\right), & 0<x / c_{0}<2 / 3 \\ f(x / c(r)), & 2 / 3<x / c(r)<1\end{cases}
$$

where the chord is a function of $\operatorname{span} r$, i.e., $c(r)=c_{0}+h \sin (2 \pi r / \lambda)$ and $x$ varies between 0 at the trailing edge and $x=c(r)$ at the leading edge.

Note that in this chapter the serration amplitude $(h)$ is normalized on the mean chord $c_{0}$ for convenience although there is no evidence to suggest that this is a meaningful parameter 


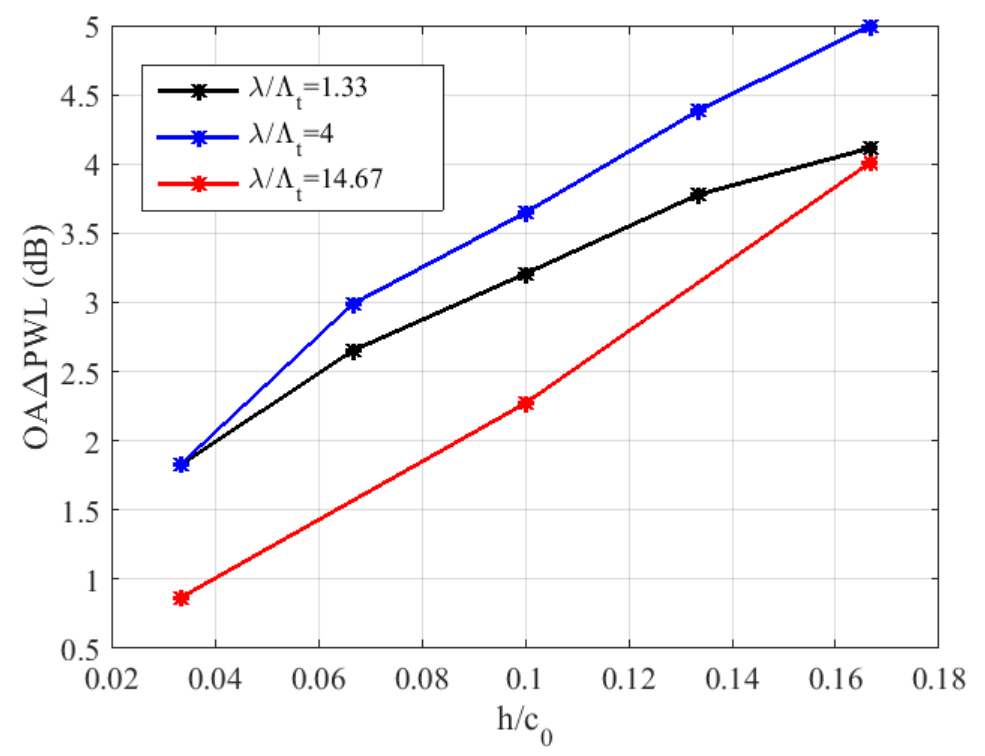

FIGURE 4.2: Effect of serrated amplitude on overall noise reductions $(O A \Delta P W L)$

in determining noise reductions. The mean chord and span of the aerofoils are $15 \mathrm{~cm}$ and $45 \mathrm{~cm}$, respectively. Details of the measurement facility and procedure are presented in the Appendix A.

\subsection{Acoustic Performance}

\subsubsection{Overall acoustic performance on flat-plates}

We start with an initial survey of the effectiveness and behaviour of leading edge serrations on the noise reductions applied to flat plates. Figure 4.2 is a plot of the overall sound power reduction integrated over the non-dimensional frequency range $\left(f c_{0} / U\right)$ between 1 to 10 for three different serration wavelengths $\lambda / \Lambda_{t}$ of 1.33, 4 and 14.67. An important observation is that largest noise reductions are obtained for the serration wavelength $\lambda / \Lambda_{t}=4$. We demonstrate in section 4.4 .3 below that this serration wavelength is close to the optimum serration wavelength at which maximum noise reductions occur. At the optimum wavelength a similarity condition is established whereby the noise reduction spectra are only a function of the non-dimensional frequency $f h / U$ suggesting that noise reductions are solely governed by the ratio of serration amplitude to the hydrodynamic wavelength $U / f$.

\subsubsection{Sound power reductions versus non-dimensional frequency $f h / U$}

In this section we present an overview of the sound power reduction spectra for varying serration amplitudes $h$ and serration wavelengths $\lambda / \Lambda_{t}$ for a turbulent in-flow with an transverse integral length scale of $3.75 \mathrm{~mm}$ and turbulence intensity of $2.5 \%$. Figures $4.3 \mathrm{a}-\mathrm{c}$ show the sound power reduction spectra plotted against non-dimensional frequency $f h / U$ for 5 different serration wavelengths ranging from 0.8 to 80 at a jet velocity $60 \mathrm{~m} / \mathrm{s}$ at the three serration amplitudes $\left(h / c_{0}\right)$ of $0.033,0.1 \& 0.167$ respectively. 


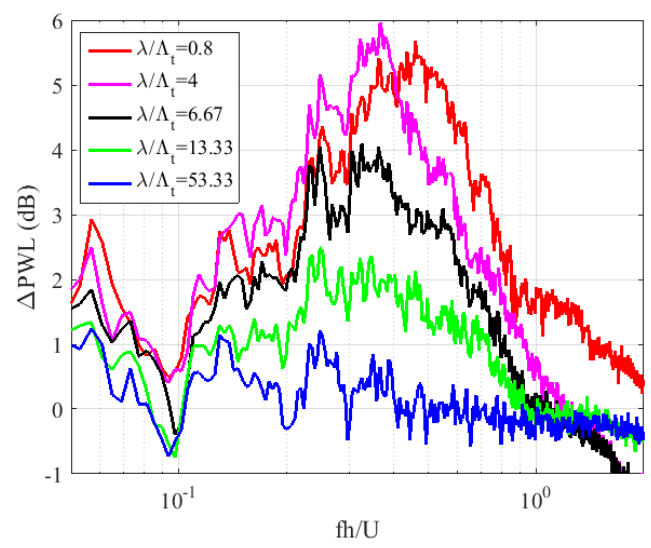

(a) $h / c_{0}=0.033$

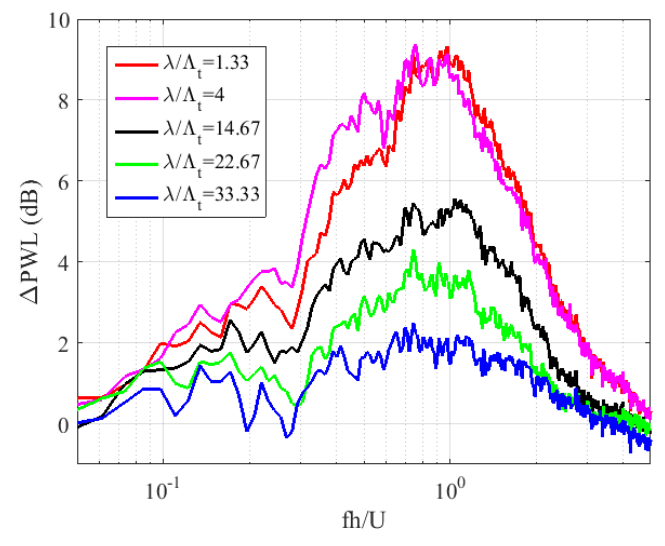

(b) $h / c_{0}=0.1$

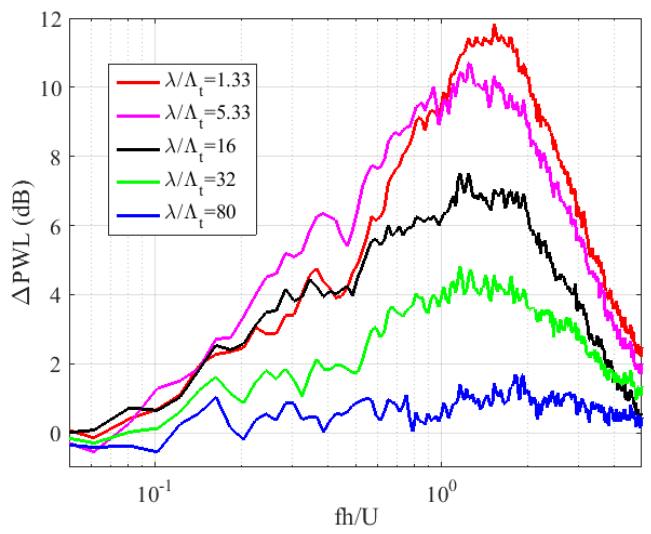

(c) $h / c_{0}=0.167$

FIGURE 4.3: Sound power reduction level $(\triangle P W L)$ for various serrated amplitude $\left(h / c_{0}\right)$ at various serration wavelength $\left(\lambda / \Lambda_{t}\right), U=60 \mathrm{~m} / \mathrm{s}$

Reductions in sound power are generally observed to increase with decreasing normalised serration wavelength $\lambda / \Lambda_{t}$ up to about 4 . Below this normalised wavelength the noise reduction spectra reduce at low frequencies suggesting the existence of an optimal serration wavelength, which we discuss further in Section 4.4.3. Sound power reductions are generally observed to increase with increasing non-dimensional frequency $f h / U$ up to some frequency at which self-noise starts to dominate. This finding was previously reported by Narayanan et al. (2015) on flat plates.

\subsubsection{Optimal serration wavelength}

We first present in figure 4.4a the measured variation in overall sound power reductions integrated over the non-dimensional frequency $\left(f c_{0} / U\right)$ band between 1 to 10 , versus serration wavelength normalised on turbulence length scale, $\lambda / \Lambda_{t}$ for five different serration amplitudes $h$. Here, $U=60 \mathrm{~m} / \mathrm{s}$ and the turbulent in-flow has an transverse integral length scale $\Lambda_{t}$ of $3.75 \mathrm{~mm}$ and a turbulence intensity of $2.5 \%$. This figure suggests the existence of an optimum non-dimensional serration wavelength $\lambda_{o} / \Lambda_{t} \approx 4$ at which maximum noise reductions occur. It is particularly well defined for the larger amplitude serrations where noise 


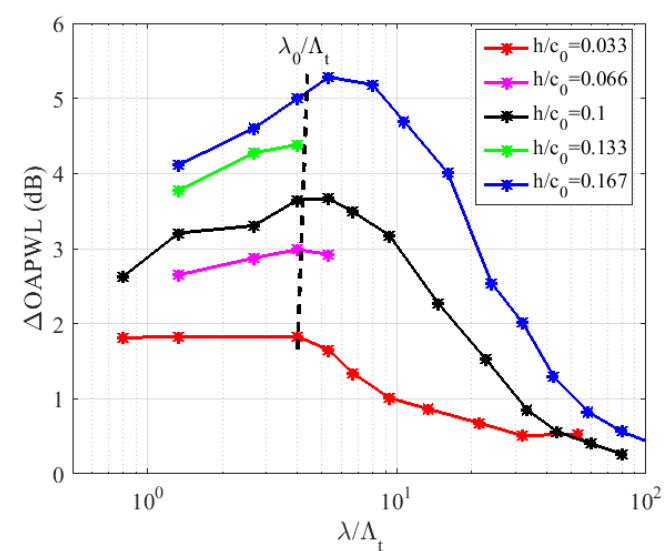

(a) At various serration amplitude $\left(h / c_{0}\right)$ for different serration wavelengths $\lambda / \Lambda_{t}$ at fixed jet velocity $60 \mathrm{~m} / \mathrm{s}$

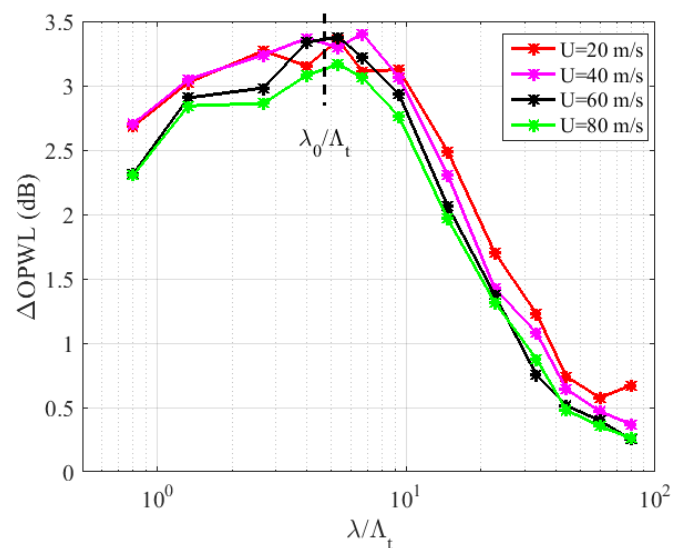

(b) Plotted against serration wavelength $\lambda / \Lambda_{t}$ for serrated amplitude $\left(h / c_{0}\right)$ of 0.1 at jet velocities varying from 20 to 80 $\mathrm{m} / \mathrm{s}$

FIGURE 4.4: Overall Sound power reduction level $(O A \Delta P W L)$ trends

reductions are much greater. The reason for this value as an optimum ratio is explained in detail in the next section.

In figure $4.4 \mathrm{~b}$, overall noise reductions integrated over non-dimensional frequency $(f h / U)$ 0.1 to 0.5 are plotted against serration wavelength normalised on turbulence length scale $\lambda / \Lambda_{t}$ at each of the four flow speeds: $20,40,60$ and $80 \mathrm{~m} / \mathrm{s}$ for a fixed serration amplitude $h / c_{0}$ of 0.1 . This figure reveals two important principles. The first arises from the collapse of the noise reduction spectra which is better than $0.5 \mathrm{~dB}$ suggesting that noise reductions are a function of non-dimensional frequency $f l / U$ where $l$ is some linear dimension related to the serration geometry that remains to be determined. The second is that the optimum serration wavelength $\lambda_{o} / \Lambda_{t} \approx 4$ is almost independent of flow speed thereby confirming the generality of this optimum value.

We now confirm the generality of $\lambda_{o} / \Lambda_{t} \approx 4$ as the optimum condition for maximum noise reductions. The measured variation in noise level in a frequency bandwidth $f h / U$ of 0.1 to 0.5 , versus $\lambda / \Lambda_{t}$ plotted logarithmically at the two different length-scales $\Lambda_{t} / c_{0}$ of 0.045 and 0.025 is plotted in figures $4.5 \mathrm{a}$. The optimum wavelength at these length scales remains around 4 . Furthermore, the optimum value appears to be well defined on this logarithmic scale in the sense that overall noise reductions diminish quite sharply for serration wavelengths lower and higher than the optimum value.

In figure $4.5 \mathrm{~b}$ we provide further for the existence of a similarity condition at the optimum wavelength $\lambda_{o} / \Lambda_{t} \approx 4$ in which the sound power reduction spectra are plotted versus Strouhal number $S t_{h}$ for the same value of $\lambda_{o} / \Lambda_{t}=4$ for the two length scales. Thus, in this comparison the ratio of turbulence length scale to serration wavelength $\lambda_{o} / \Lambda_{t}$ and the ratio of serration amplitude to hydrodynamic wavelength, $f h / U$, are identical. The sound power reduction spectra are remarkably similar at frequencies up to which self-noise starts to dominate thereby providing compelling evidence for the existence of self-similarity in this interaction process.

Finally, we present the sensitivity of the sound power reduction spectra to serration amplitude and serration wavelength at a single turbulence integral length-scale. Sound power reductions are plotted in figures 4.6a and c versus $S t_{h}$ for the two sub-optimal serration wavelengths of $\lambda / \Lambda_{t}=1.33$ and 10, and at the optimum wavelength of 4 in figure 4.6b. Each 


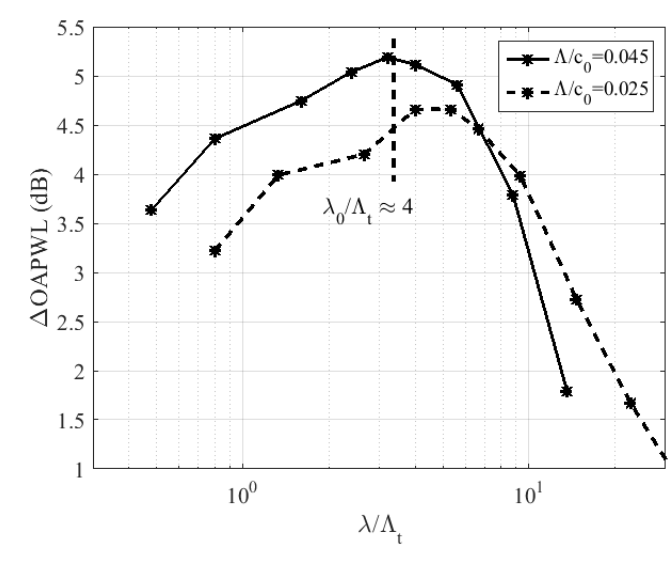

(a) Overall power level reduction

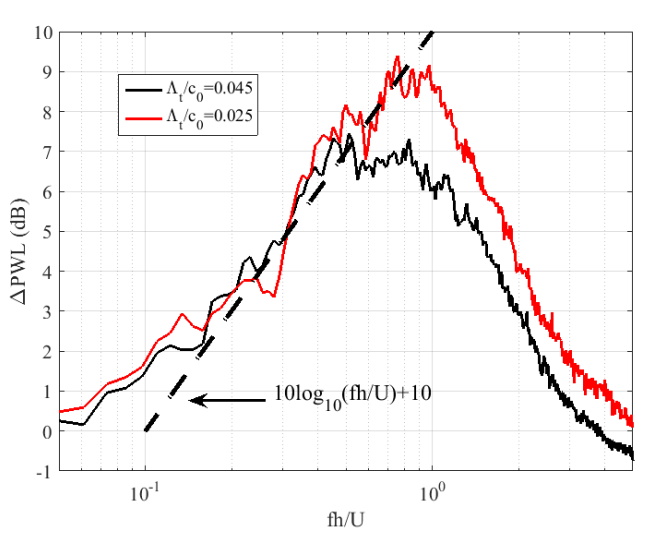

(b) Noise reductions, $\lambda / \Lambda_{t} \approx 4$

FIGURE 4.5: Influence of integral length scale $\left(\Lambda_{t} / c_{0}\right)$ on noise reductions

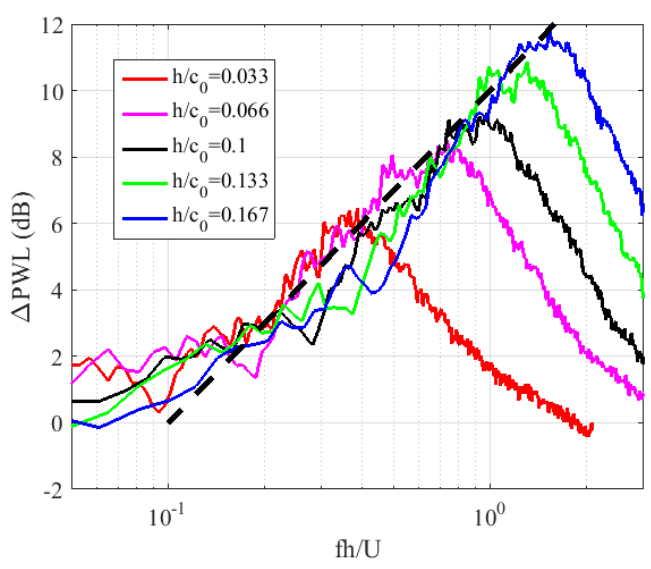

(a) $\lambda / \Lambda_{t} \approx 1.33$

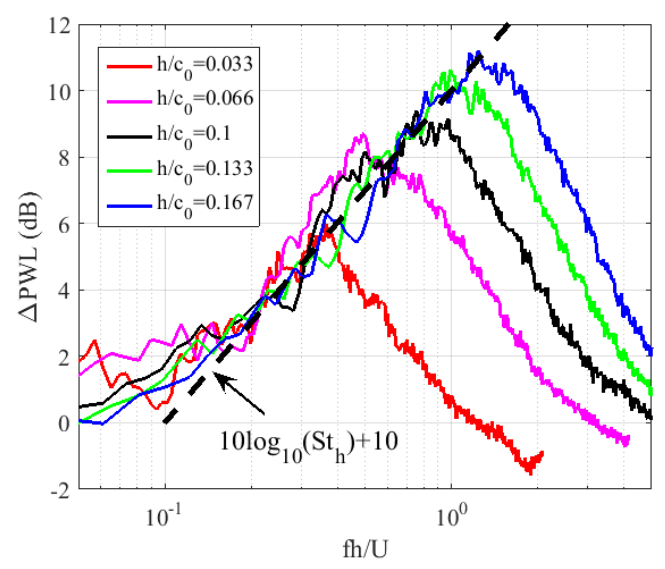

(b) $\lambda / \Lambda_{t} \approx 4.5$

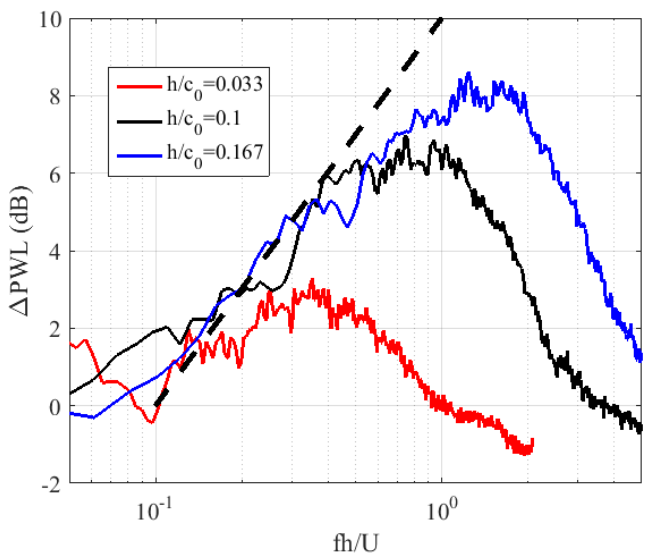

(c) $\lambda / \Lambda_{t} \approx 10$

FIGURE 4.6: Sound power reductions for various serration amplitudes at three different serration wavelength $\lambda / \Lambda_{t}$ of $1.33,4.5,10$ and jet velocity $U=60 \mathrm{~m} / \mathrm{s}$ 
figure is plotted at different serration amplitudes. It is clear that at the optimum wavelength $\lambda / \Lambda_{t} \approx 4$ the noise reduction spectra plotted in figure $4.6 \mathrm{~b}$ collapse to within $1 \mathrm{~dB}$ suggesting that noise reductions are solely a function of Strouhal number $S t_{h}$ in the frequency range where leading edge noise is dominant and self-noise can be neglected. No such collapse is observed for the wide serration $\lambda / \Lambda_{t}=10$ and perceptibly worse collapse for the narrower serration $\lambda / \Lambda_{t}=1.33$. At the optimum serration wavelength, therefore, the noise reduction spectra plotted in figure $4.6 \mathrm{~b}$ follow a similarity principle since they are determined solely by the ratio of hydrodynamic wavelength $U / f$ to the serration amplitude $h$. Moreover, for this optimum geometry, the variation in sound power reduction level is observed to closely follow $10 \log _{10}\left(S t_{h}\right)+10(\mathrm{~dB})$ and therefore represents an upper limit on the sound power reductions obtainable using single-wavelength leading edge serrations. In terms of the ratio of sound power due to the serrated aerofoil $W_{s}$ and the baseline aerofoil $W_{b l}$, this dependence corresponds to an inverse Strouhal number scaling of $W_{s} / W_{b l} \propto 1 / S t_{h}$. A model aimed at explaining this behaviour is presented in section 4.4.4.

Figure 4.6 provides further confirmation of the existence of an optimum serration wavelength $\lambda_{o} / \Lambda_{t} \approx 4$ at which maximum noise reductions occur.

The sound power noise reduction spectra $10 \log _{10}\left(W_{b l} / W_{s}\right)$ presented above at the optimum serration wavelength $\lambda_{o}$ and non-optimum wavelengths follow different frequency scaling, which may be summarized in 4.2 as follows:

$$
\frac{W_{s}(\omega)}{W_{b l}(\omega)}=f\left(\frac{f l}{U}, \frac{\lambda}{\Lambda_{t}}\right), \quad\left\{\begin{array}{l}
l=h \quad \text { for } \lambda=\lambda_{o} \\
l=\text { const. } \text { for } \lambda \neq \lambda_{o}
\end{array}\right.
$$

\subsubsection{Interpretation}

In the next section, we exploit the similarity behaviour observed at the optimum serration wavelength to develop a simple model aimed at explaining the inverse Strouhal dependence observed in figure 4.6.

\section{Derivation of optimum wavelength $\lambda_{0}=4 \Lambda_{t}$}

Previously we have shown that optimum noise reductions are obtained when the serration wavelength $\lambda_{o} \approx 4 \Lambda_{t}$. Previously Kim et al. (2016) have demonstrated that compact sources at the valleys are the dominant noise source on a serrated leading edge. In this section, we use this finding to provide the explanation for this relationship. An aerofoil with serration wavelength $\lambda$ provides $N_{r}=L / \lambda$ equally distributed compact sources over the aerofoil span located at the valley positions separated one wavelength $\lambda$ apart. For simplicity, if the path length differences between each compact source and a far field observer is neglected (since adjacent sources are generally much closer together than the acoustic wavelength), retarded time differences can be ignored and the total radiated pressure at time $t$ is proportional to the sum of source strengths $q\left(r_{n}, t\right)$ (with time delay also ignored) where $r_{n}$ is the position along the span on the $n^{\text {th }}$ valley source, i.e.,

$$
p(t) \propto \sum_{n=1}^{N_{r}} q\left(r_{n}, t\right)
$$


where $r_{n}=n \lambda$

The radiated sound power $W_{s}$ is related to the mean square far-field pressure integrated over some suitable closed surface. The overall mean square pressure will depend on the degree of correlation between adjacent sources on the serrated leading edge, which of course is related to the serration wavelength compared to the turbulence integral length scale. Following equation 4.3 , the far-field mean square pressure, and hence radiated sound power, can therefore be written as,

$$
W_{s} \propto \overline{p^{2}} \propto \sum_{n=1}^{N_{r}} \sum_{n^{\prime}=1}^{N_{r}} \overline{q\left(r_{n}, t\right) q\left(r_{n^{\prime}}, t\right)}
$$

where $W_{s}$ and $\overline{p^{2}}$ represents the integration over all spectral component, $\int_{-\infty}^{\infty} W_{s}(\omega) d \omega$ and $\int_{-\infty}^{\infty} \overline{p^{2}}(\omega) d \omega$. Assuming that the source strength at each valley is identical and that the spatial correlation coefficient $\rho$ between the compact source strengths at the valleys is only a function of the separation distance between them i.e. $\rho\left(r_{n}, r_{n^{\prime}}\right)=\rho\left(\left|n-n^{\prime}\right| \lambda\right)$, (which is the case when excited by homogeneous turbulence), the source strength spatial correlations in equation 4.4 can be written as,

$$
\overline{q\left(r_{n}, t\right) q\left(r_{n^{\prime}}, t\right)}=\overline{q^{2}} \rho\left(\left|n-n^{\prime}\right| \lambda\right)
$$

where $\overline{q^{2}}=\overline{q^{2}}\left(r_{n}, t\right)$. In this analysis we adopt a highly simplified model for the spatial correlation coefficient function defined in terms of the turbulence integral length scale $\Lambda_{t}$, $\rho\left(\left|n-n^{\prime}\right| \lambda\right)=e^{-\left|n-n^{\prime}\right| \lambda / \Lambda_{t}}$. The radiated sound power can now be written in the form,

$$
W_{s} \propto \overline{q^{2}} \sum_{n=1}^{N_{r}} \sum_{n^{\prime}=1}^{N_{r}} e^{-\left|n-n^{\prime}\right| \lambda / \Lambda_{t}}
$$

Finally, expanding the terms in equation 4.6 and replacing $\overline{q^{2}}$ by $W_{r}$ the sound power produced by a single valley source radiating in isolation, leads to equation 4.7 for the total sound power radiated by the serrated leading edge excited by turbulence with length scale $\Lambda_{t}$, divided by the sound power radiated by $N_{r}$ valley sources radiating incoherently,

$$
\frac{W_{s}}{W_{r} N_{r}}=\left[1+2 \sum_{n=1}^{N_{r}-1}\left(1-n / N_{r}\right) e^{-n \lambda / \Lambda_{t}}\right]
$$

One interpretation of this power ratio is that it represent the ratio of coherent valley sources to incoherent valley sources (i.e., when the serration wavelength is much larger than the turbulence integral length scale). Equation 4.7 is plotted below in Figure 4.7 as a function of $\lambda / \Lambda_{t}$ for the three cases, $N_{r}=10,20$ and 30 .

This figure clearly reveals the significance of turbulence length scale to the effectiveness of the noise reductions obtained using leading edge serrations. The figure clearly defines a minimum serration wavelength, $\lambda_{o} / \Lambda_{t} \approx 4$, below which adjacent sources are excited coherently, leading to constructive interference in the far-field, and hence provide relatively poor noise reductions. In the small wavelength limit (compared to length scale), the sound power ratio tends to $N_{r}$, i.e., the sound power from the serrated aerofoil radiates as $N_{r}^{2}$, since all $N_{r}$ sources radiate in-phase. Above this value, $\lambda_{o} / \Lambda_{t} \approx 4$, the sound power ratio 


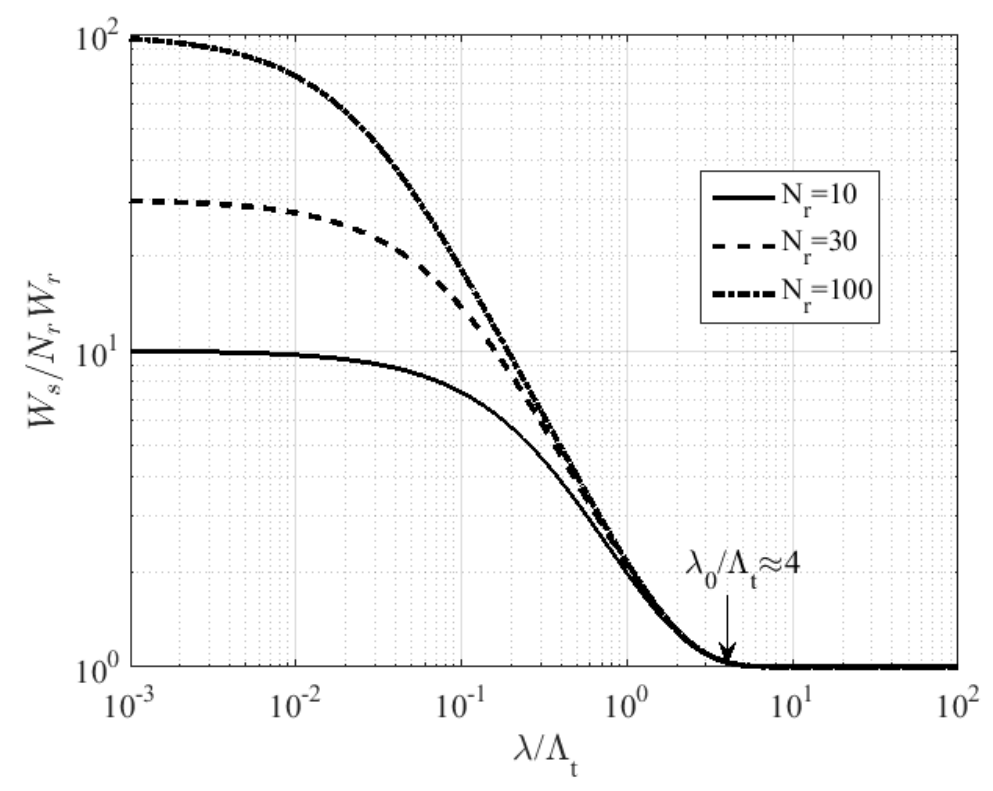

FIGURE 4.7: Variation in total sound power versus $\lambda / \Lambda_{t}$

tends to 1, indicating that all source are excited incoherently. Note that this limit appears to be independent of $N_{r}$. Precisely this value of 4 is identified in figure 4.4 as the optimum serrated wavelength. The optimum condition for maximum noise reductions, therefore, occurs when adjacent sources are only just excited incoherently. Note that the coherence length $l(\omega)<\Lambda$, therefore the condition $\lambda_{o} / \Lambda_{t} \approx 4$ assumes uncorrelated sources at all frequencies.

Also note that a good approximation to equation 4.7 in the vicinity of $\lambda_{o} / \Lambda_{t} \approx 4$ can be obtained by including only contributions from adjacent valley sources since pairs of sources further than the nearest neighbour have negligible correlation coefficient. At the optimum serration wavelength, therefore, coherent interaction between valley sources only occurs between adjacent sources.

\section{Geometric similarity interpretation of Strouhal number scaling}

The previous section has shown both experimentally and theoretically that maximum noise reductions are obtained when $\lambda_{o} / \Lambda_{t} \approx 4$. One of the main findings of this chapter is that at the optimum wavelength $\lambda_{o}$, the sound power reduction spectra collapse on the nondimensional frequency $S t_{h}$, which may be interpreted as the ratio of serration height $h$ to hydrodynamic wavelength $\lambda_{h}=U / f$. This finding suggests the existence of a geometric similarity condition in which the sound power reduction is only as a function of these two length scales. In this section, we demonstrate that this finding is consistent with the hypothesis that the length $l\left(\omega, h, \Lambda_{t}\right)$ of the sources along the sinusoidal leading scale linearly with the hydrodynamic wavelength.

In this analysis the length $l$ of the source along the sinusoidal leading edge is expressed as,

$$
l\left(\omega, h, \Lambda_{t}\right)=\eta\left(h, \Lambda_{t}\right) \lambda_{h}(\omega)
$$


where $\eta$ is a non-dimensional constant of proportionality that is dependent on the transverse turbulence length scale and serration height. Another important finding of the numerical simulations of Kim et al. (2016) is that the source strength $\varpi(\omega)$ (surface pressure per unit edge length) at the valley at all frequencies is similar to that of the straight leading edge. As shown in section 4.4.4, at the optimum serration wavelength the total sound power radiation $W_{s}$ equals the sound power per valley $W_{r}$ multiplied by the number of valleys $N_{r}$, i.e., $W_{s}(\omega)=N_{r}(\lambda) W_{r}(\omega)$. The radiated sound power for each valley is assumed here to be equal to the 'length' $l$ of the source along the sinusoidal leading edge and the sound power per unit length $\varpi$, i.e.,

$$
W_{r}(\omega)=\varpi(\omega) l\left(\omega, h, \Lambda_{t}\right)
$$

where, $l$ is the length of the source given by equation 4.8 which is assumed to be proportional to $\lambda_{h}$ and $N_{r}(\lambda)=L / \lambda$, where $L$ is the aerofoil span. Following an identical argument the noise power from the straight baseline leading edge is simply,

$$
W_{b l}(\omega)=\varpi(\omega) L
$$

The ratio of the sound power of the serrated aerofoil to the straight leading edge is therefore,

$$
\frac{W_{s}(\omega)}{W_{b l}(\omega)}=\frac{\eta\left(h, \Lambda_{t}\right) \lambda_{h}(\omega)}{\lambda_{o}}
$$

Finally, expressing $\lambda_{o}$ in terms of serration height and angle $\theta_{o}=\tan ^{-1}\left(4 h / \lambda_{o}\right)$, the power ratio may be written as

$$
\frac{W_{s}(\omega)}{W_{b l}(\omega)}=\frac{\eta\left(h, \Lambda_{t}\right) \tan \left(\theta_{o}\right)}{4 S t_{h}} \propto \frac{1}{S t_{h}}
$$

where $S t_{h}$ is the Strouhal number based on serration height $S t_{h}=f h / U$, as observed in the experimental data at the optimum wavelength.

\section{Phase model for the prediction noise reduction spectra at the optimum wavelength}

The simple scaling-law analysis above has provided a framework for understanding the inverse Strouhal number behaviour observed conclusively in figures 4.5 and 4.6. In this section we present a very simple, idealised model to provide a partial explanation for the constant of proportionality in equation 4.12 , which is approximately 0.1 . It is emphasize that this is not a complete model for predicting the noise reductions from the serrated leading edge, which may be found in the recent paper by Lyu et al. (2016), but provide a simple physical basis for understanding the noise reduction spectra, which was found to be of the form $10 \log _{10}\left(S t_{h}\right)+10$ at the optimum serration wavelength. A schematic of the parallel gust impinging on the serration leading edge is shown in figure 4.8. In the analysis we make the following assumptions:

1. The edge is excited coherently, i.e., the serration wavelength is equal to its optimum value.

2. The surface pressure response is concentrated along the leading edge. 


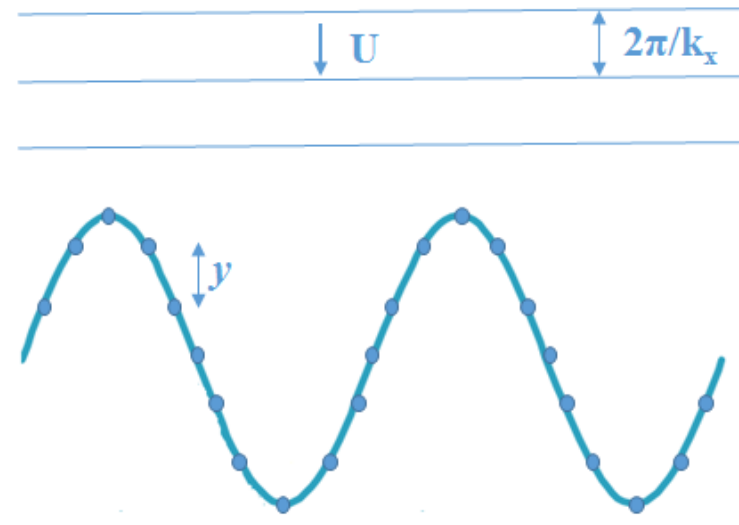

Phase difference $=\omega y / U$

FIGURE 4.8: Parallel gust incident on a sinusoidal leading edge

3. Phase compatibility is assumed in which the phase of the surface pressure response along the leading edge is identical to that of the impinging gusts along the leading edge.

4. The length of the sources in the streamwise (flow) direction is identical to that of the flat plate.

5. The unsteady aerofoil surface response is dominated by the gust component convecting parallel to the flow direction.

We write the far field sound Power Spectra $W_{s}(\omega)$ in terms of the transverse vortical gust component convecting parallel to the flow direction of the form, i.e.,

$$
W_{s}(\omega)=\overline{v^{2}}(\omega) S(\omega) R(\omega)
$$

where $\overline{v^{2}}(\omega)$ is the mean square transverse turbulence velocity of the normally incident gust at frequency $\omega, S(\omega)$ is the sound power per unit mean square velocity $\overline{v^{2}}(\omega)$ at the leading edge of the baseline aerofoil, and $R(\omega)$ is the sound power reduction coefficient $(0 \leq|R| \leq 1)$ due to the serrated aerofoil. For simplicity, given the basic assumptions listed above, the sound power reduction coefficient is predicted by integrating the phase variation $\omega h \cos (\theta) / U$ along one cycle of the sinusoidal leading edge, i.e.,

$$
R(\omega)=\left|\frac{1}{2 \pi} \int_{0}^{2 \pi} e^{-i \omega h \cos (\theta) / U} d \theta\right|^{2}
$$

This integral expression evaluates to

$$
R(\omega)=J_{0}^{2}(\omega h / U)
$$

where $J_{0}$ is the Bessel function of the first kind of order 0 . This simple expression for the sound power of the leading edge serration is now compared to that for the baseline leading edge $h=0$, which in equation 4.14 becomes 1 . 


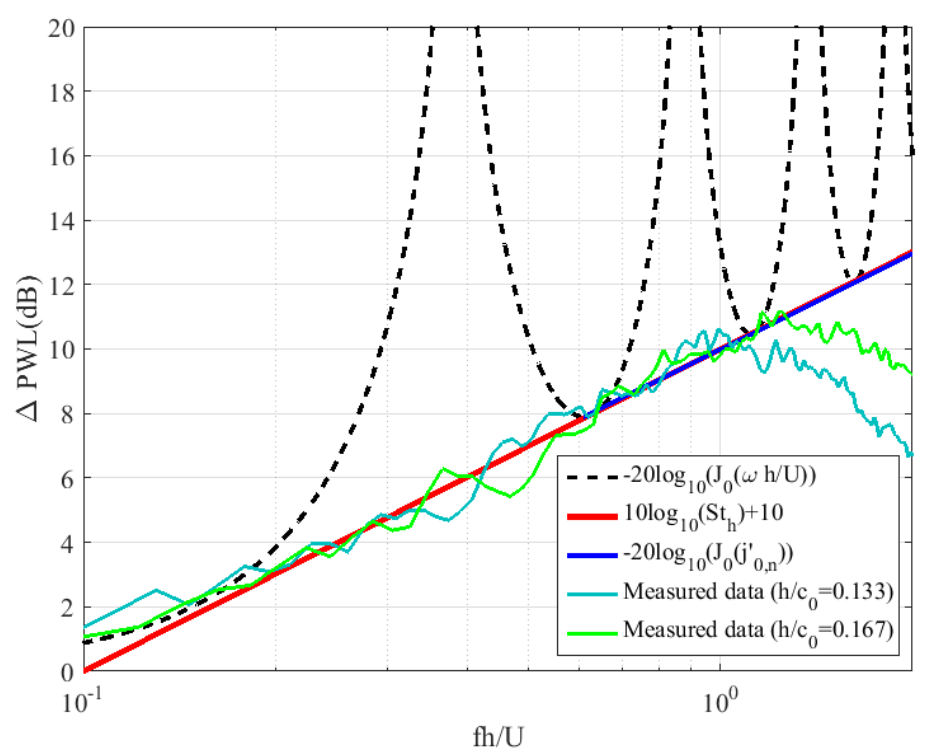

FIGURE 4.9: Comparison of simple analytical model against experiments at optimum serration angle.

The sound power level reduction predicted by the very simple, single-gust model is therefore given by

$$
\Delta P W L(\omega)=10 \log _{10}\left(W_{s}(\omega) / W_{b l}(\omega)\right)=-20 \log _{10}\left(J_{0}(\omega h / U)\right)
$$

Figure 4.9 provides a comparison of the measured noise reduction spectra with the simple analytical expression of equation 4.16. Also plotted is the line $10 \log _{10}\left(S t_{h}\right)+10$ obtained by observation of the measured spectra. Excellent agreement is obtained from all three curves at the stationary values of $J_{0}(\omega h / U)$. At all other frequencies, agreement is poor, particularly at the zeros of $J_{0}(\omega h / U)$, where complete cancellation of the leading edge response is predicted. Clearly, the major deficiency with this simple single-gust model is the assumption of uniform source strengths along the leading edge. As shown by (Clair et al., 2013; Kim et al., 2016) for example the source strengths along the hill region is generally weaker due to the combined effect of gust components becoming cut-off and a smaller mean flow component in the direction normal to the edge (Kim et al. (2016)). However, this simple single-gust model is nevertheless useful for speculating on the reasons underlying the trend in the noise reduction spectra. The model, therefore, predicts the maximum noise reductions for a sinusoidal serration. In practice, however, noise reductions are considerably less than this value due to the presence of multiple oblique gusts, imperfect phase cancellation along the leading edge and partial correlation of the blade response along the leading edge due to the finite eddy size. A similar model has been developed in chapter 7 with coherence and the results are comparatively close to $10 \log _{10}\left(S t_{h}\right)+10$.

\section{Balance between source strength per valley and number of valleys}

Figures 4.5 and 4.6 provide strong experimental evidence for the existence of an optimum serration wavelength at which maximum noise reductions occur. In section 4.4 .3 , this has been directly linked to the turbulence integral length-scale. In this section, we provide an 
alternative, but consistent, interpretation of the optimum serration wavelength as the wavelength that provides the optimum combination of source strength per valley and the number of valleys. For serration wavelengths greater than the optimum wavelength, the valley sources radiate incoherently and hence the sound power due to the serrated aerofoil can be written as the product of the sound power per valley $W_{r}$ and the number of valleys, which is simply $N_{r}=L / \lambda$, i.e.,

$$
W_{s}=W_{r}(\lambda) \frac{L}{\lambda}
$$

Note that $W_{r}$ could be sound power at a single frequency or integrated over a frequency bandwidth. The optimum serration wavelength $\lambda=\lambda_{o}$ occurs when

$$
\left.\frac{d W_{s}}{d \lambda}\right|_{\lambda=\lambda_{o}}=0
$$

The solution to equation 4.18 gives the condition for the optimum wavelength of the form,

$$
\left.\frac{1}{W_{r}} \frac{d W_{r}}{d \lambda}\right|_{\lambda=\lambda_{o}}=\left.\frac{1}{\lambda}\right|_{\lambda=\lambda_{o}}
$$

which says that the optimum serration wavelength $\lambda_{o}$ occurs when the rate of change of sound power per valley with $\lambda$ exactly equals the variation in the number of valleys with $\lambda$. This optimum condition for $\lambda_{o}$ is shown explicitly in Figure 4.10, which is a plot of the sound power level per valley measured at $U=60 \mathrm{~m} / \mathrm{s}, h / c_{0}=0.1$ versus $\lambda$. Also, shown is the line $-10 \log _{10}\left(N_{r}\right)+67.75$ showing the number of valleys per unit span in $\mathrm{dB}$. Note that the constant $67.75 \mathrm{~dB}$ has been added to allow comparison of the two curves. At the optimum wavelength, the gradients of the two curves match, in agreement with equation 4.19.

For serration wavelengths less than the optimum value the sound power per valley increases at a slower rate with increasing $\lambda$ than the number of valleys increases. This is a result of the coherence effect discussed in detail in section 4.4.4, whereby coherent excitation of the valley sources causes the sound power radiation to vary at a faster rate than the number of valleys $N_{r}$. For serration wavelengths greater than the optimum, the valleys continue to radiate incoherently but clearly, the source strength must be increasing at a faster rate than $N_{r}$. The optimum serration wavelength, therefore, occurs when the rate of change of sound power with $\lambda$ increases at precisely the same rate as $N_{r}$ itself.

\subsubsection{Noise reduction contours}

In this section, noise reductions contours due to LE serrations are discussed. Figure 4.11 shows the noise reductions contours for varying serration wavelength $\lambda / \Lambda_{t}$ at three different serration amplitudes of $h / c_{0}$ of $0.033,0.1 \& 0.167$ and a fixed jet velocity, $U=60 \mathrm{~m} / \mathrm{s}$. Clearly around optimum serration wavelength as identified in section 4.4.3, the serrations are effective, i.e., significant noise reductions $(3 \mathrm{~dB})$ are observed. As described previously, at a non-dimensional frequency of $f h / U$ of 0.2 , we get significant noise reductions. The range of serration wavelengths $\lambda / \Lambda_{t}$ where $3 \mathrm{~dB}$ of noise reductions are achieved for $f h / U=0.2$ is observed to increase with increase of serration amplitude $h / c_{0}$. 


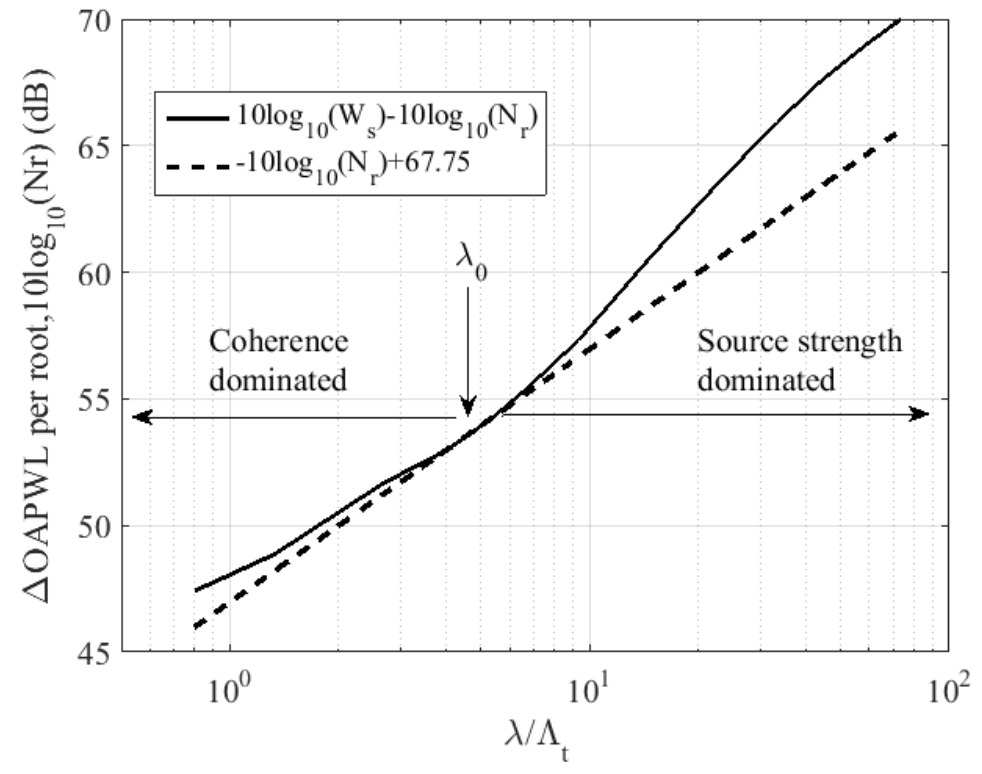

FIGURE 4.10: Balance between number of valleys and source strength

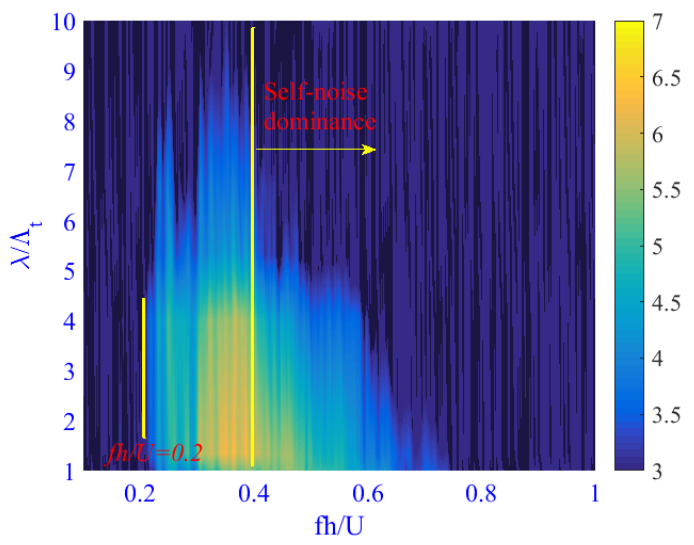

(a) $h / c_{0}=0.03$

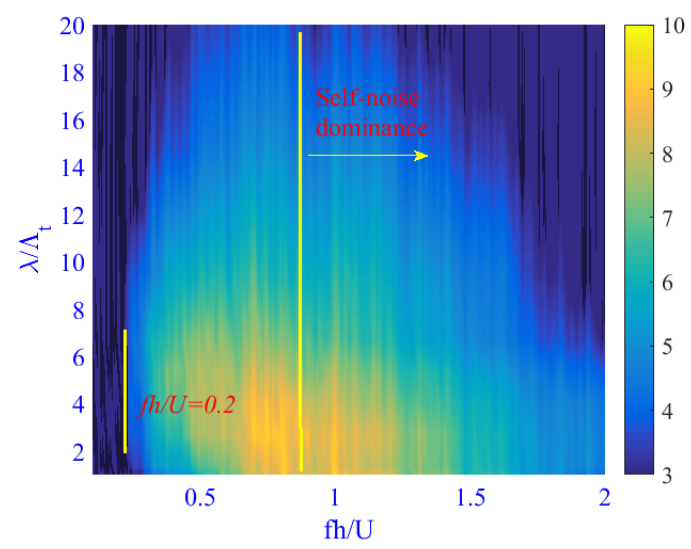

(b) $h / c_{0}=0.1$

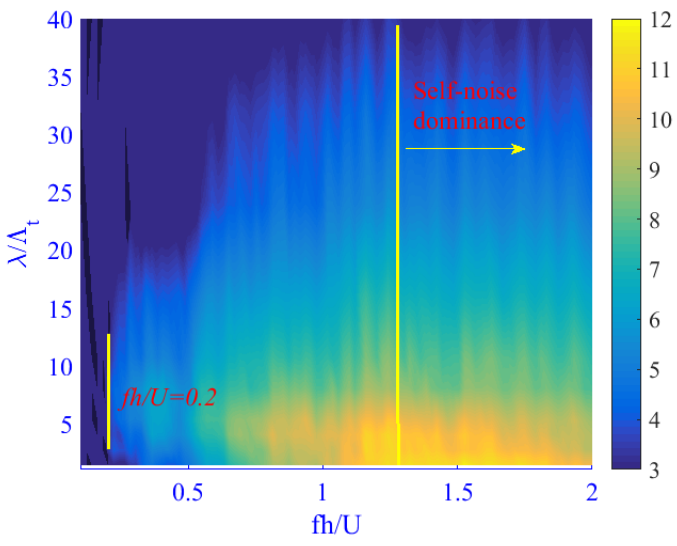

(c) $h / c_{0}=0.167$

FIGURE 4.11: Sound power reduction contours $(\triangle P W L)$ for Jet Velocity, $U=$ $60 \mathrm{~m} / \mathrm{s}$ 


\subsubsection{Influence of self-noise on aerofoils on 3D aerofoils}

Work described in previous sections and in Narayanan et al. (2014, 2015); Chaitanya et al. (2015b) has characterized the behaviour and effectiveness of sinusoidal leading edge serrations on flat plates. The current section aims to verify whether the same behavior, in particular the similarity relationships observed for flat plates, also applies to leading edge serrations introduced into 3D aerofoil geometries.

The important difference between the aerodynamic noise due to a flat plate in a turbulent stream and a 3D aerofoil is a much greater contribution of trailing edge self-noise due to a more energetic boundary layer forming over the surface of the 3D aerofoil driven by the adverse pressure gradient. Another important difference between the serration on an aerofoil and a flat plate is the effects of aerofoil leading edge geometry which the effect of distorting the incoming turbulence due to mean flow gradients. This section aims to investigate the balance between the self noise and leading edge noise, furthermore, to assess the effect of leading edge serrations on the trailing edge self-noise.

Figure 4.12 shows the total radiated sound power level spectra for the baseline aerofoil plotted against non-dimensionless frequencies at the jet velocities of 20 and $60 \mathrm{~m} / \mathrm{s}$. Also shown is the spectra of radiated noise due to TE self-noise alone obtained by removing the grid. Comparison between the two spectra confirms that interaction noise, even with serrations, is the dominant noise source at low frequencies whilst self-noise dominates at high frequencies. As also shown in Narayanan et al. (2015) this figure makes clear that self-noise is the factor that limits noise reductions by the serrations at high frequencies. The frequencies at which maximum noise reductions occur appears to correspond to the frequency at which self-noise starts to become significant compared to interaction noise. This frequency of maximum noise reduction, as indicated by the vertical dashed line, appears to occur at approximately the same non-dimensional frequency $f c_{0} / U$ for both jet velocities of around $f c_{0} / U=10$ for this aerofoil geometry. Figure 4.12 also shows a reduction in self-noise for serrated leading edge (dashed blue) than compared to baseline self-noise (dashed black). This is further discussed below.

\subsubsection{Influence of leading edge serrations on trailing edge self-noise}

The effect of leading edge serrations on interaction noise and trailing edge self-noise can be determined from separate measurements of the overall noise radiation (i.e., the sum of LE, $\mathrm{TE}$ and background noise), the sum of self noise and background noise (by removing the turbulent grid), and background noise spectra alone (without aerofoil).

Figures 4.13a show the variation of the reduction in total sound power versus non-dimensionless frequency $\left(f c_{0} / U\right)$ at a fixed serration wavelength $\left(\lambda / \Lambda_{t}\right)$ of 2.67 for various serration amplitudes $\left(h / c_{0}\right)$ at a jet velocity of $60 \mathrm{~m} / \mathrm{s}$ and angle of attack of $0^{\circ}$. Note that the serration wavelength for these cases are close to the optimum serrated wavelength identified in section 4.4.3. The noise reduction spectra follows very closely the behaviour observed for flat plates. A detailed comparison between flat plate and aerofoil measured noise reductions with numerical predictions are presented in Chaitanya et al. (2015b). As observed in figure 4.13, the noise reduction spectra increases with increasing frequency until some optimum frequency of approximately $f c_{0} / U=8$, above which it then falls due to the dominance of self-noise at high frequencies. The introduction of trailing edge serration has been shown to reduce trailing edge noise, for example (Gruber, 2012; Gruber et al., 2013), which if applied to the current aerofoil will therefore extend the frequency range over which leading 


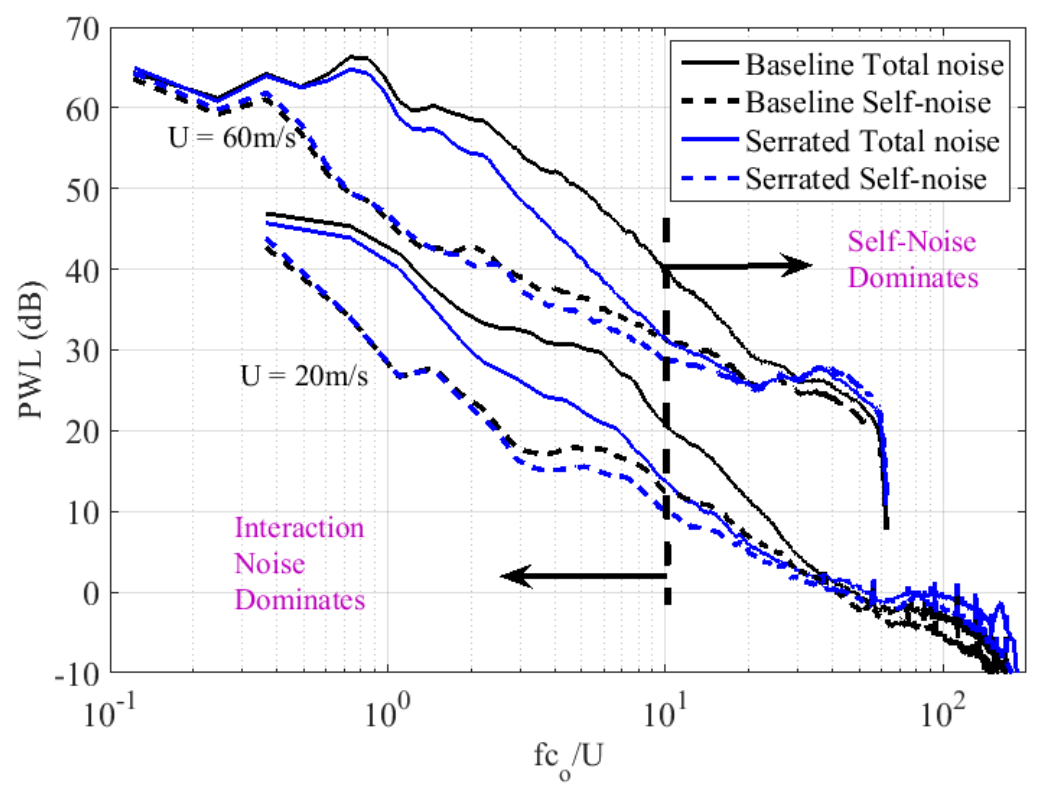

FIGURE 4.12: Power level spectra comparison between baseline NACA65 aerofoils with serrated aerofoil of amplitude $\left(h / c_{0}\right) 0.167$ and wavelength $\left(\lambda / c_{0}\right) 0.067$ along with its self-noise

edge serrations are effective. An important observation is that, in this high frequency range, the noise is sensitive to the amplitude of the leading edge serration thus clearly suggesting that leading edge serrations can reduce trailing edge noise.

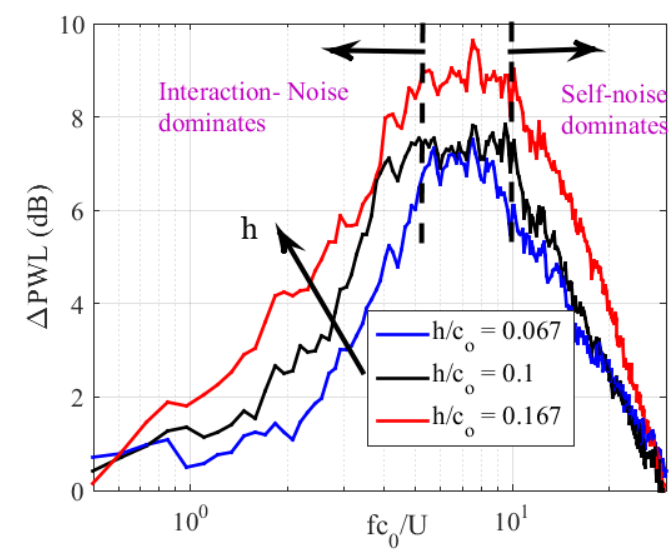

(a) Influence on total noise reduction

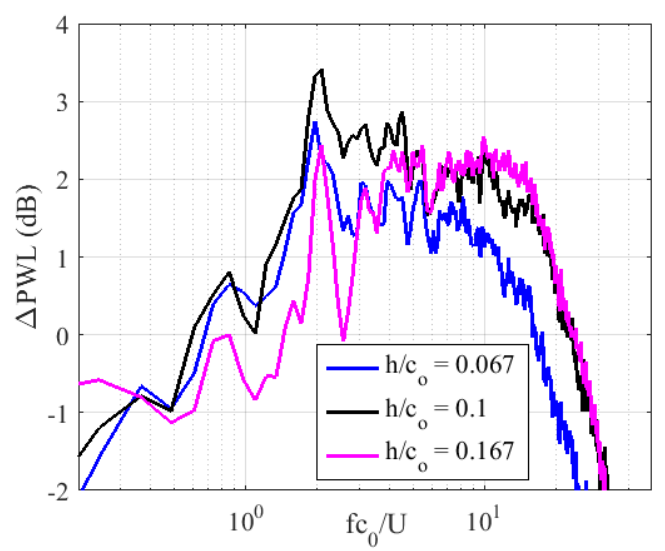

(b) Influence on self-noise reduction

FIGURE 4.13: Sound power reduction level $(\triangle P W L)$ for various serrated amplitude $(h)$ at jet velocity $60 \mathrm{~m} / \mathrm{s}$ and $A o A=0^{\circ}$ for a serrated wavelength

$\left(\lambda / c_{0}\right)$ of 0.067

Figure $4.13 \mathrm{~b}$ demonstrates this effect explicitly by showing self-noise reduction spectra due to the introduction of leading edge serrations. Reductions in self noise of up to $3 \mathrm{~dB}$ are observed, which are comparable to those obtained with trailing edge serrations (Gruber, 2012; Gruber et al., 2013). This effect is undoubtedly due to modification to the boundary layer caused by the leading edge serration. This is investigated using PIV measurements in section 4.6. 


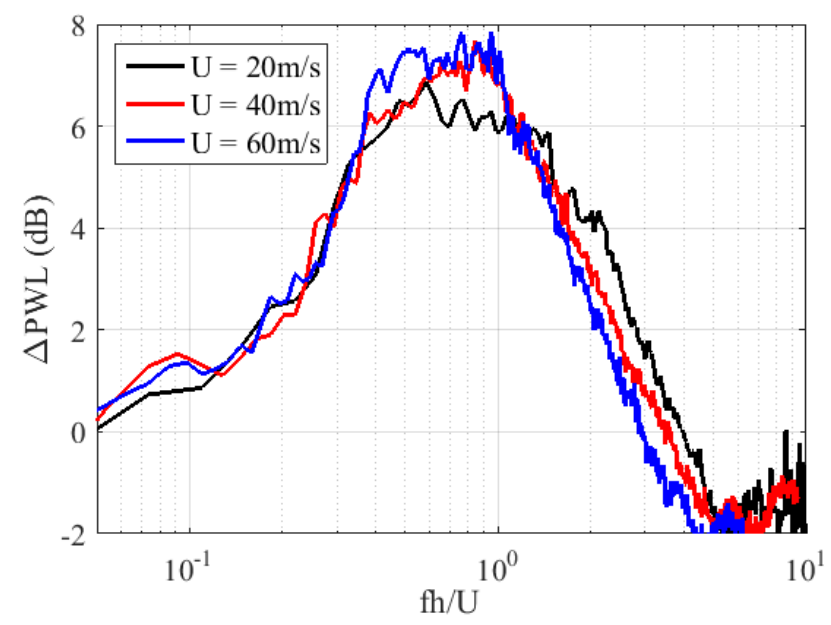

FIGURE 4.14: Sound power reduction level $(\triangle P W L)$ for serrated amplitude $\left(h / c_{0}\right)$ of 0.1 and serrated wavelength $\left(\lambda / \Lambda_{t}\right)$ of 2.67 at various velocities at $A \circ A=0^{\circ}$

We now investigate the sensitivity of noise reductions to flow speed. Figure 4.14 shows the reduction in total noise versus dimensionless frequency $(f h / U)$ at speeds of 20,40 and 60 $\mathrm{m} / \mathrm{s}$ for a serration amplitude and wavelength of $\left(h / c_{0}\right)$ of 0.1 and $\left(\lambda / \Lambda_{t}\right)$ of 2.67. As also observed in the flat plate study in section 4.4.3, the noise reduction spectra follows a nondimensional frequency dependence $(f h / U)$ in the low frequency range where interaction noise is dominant. However, at higher frequency $(f h / U>0.5)$ collapse of the spectra with non-dimensional frequency based on amplitude is relatively poorer. This finding is consistent with the dominance of self noise in this frequency range, where a more appropriate nondimensional frequency would be defined with respect to boundary layer thickness (Gruber, 2012; Gruber et al., 2013).

\subsubsection{Validity of optimal serration angles for 3D aerofoils}

In the flat plate study in Section 4.4.3, an optimum serration wavelength for maximum noise reductions was identified. At this optimum angle, the noise reduction spectra were shown to collapse on $f h / U$ and follow the linear frequency dependence $\Delta P W L=10 \log _{10}\left(S t_{h}\right)+10$. Figure 4.15 shows the noise reduction spectra for the NACA65 aerofoil at three serration angles close to the optimum angle, which can be seen to closely follow this relationship confirming that the essential noise reductions mechanisms on flat plates also apply to the aerofoil even though the flow behaviour in the vicinity of the valleys is much more complex.

The noise reduction spectra in figure 4.15 for the optimum serrations may be used to identify a frequency $f_{3 d B}$ above which noise reductions become significant $(>3 d B)$. From this figure $f_{3 d B}$ nearly corresponds to a Strouhal number of $\left(f_{3 d B} h / U\right)=0.25$. This value is consistent with the frequency identified by Narayanan et al. (2015). However, it is only valid for serrations at the optimum serration wavelengths. For sub-optimal serration wavelengths the rate of increase in noise reduction with frequency, for example, as shown in figure 4.6, is slower than for the optimum case and hence the frequency $\left(f_{3 d B}\right)$ will be higher. 


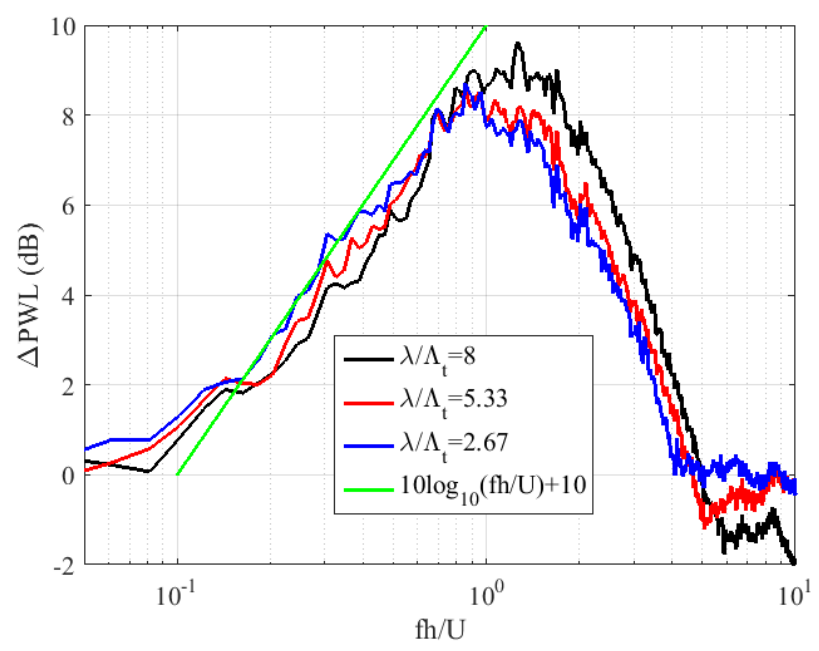

FIGURE 4.15: Sound power reduction level $(\triangle P W L)$ on NACA65 aerofoil for various inclination angle $\lambda / \Lambda_{t}$ at jet velocity $U=60 \mathrm{~m} / \mathrm{s}$ and $A o A=0^{\circ}$ for serrated amplitude $\left(h / c_{0}\right)$ of 0.167

\subsubsection{Comparison with serrated flat plate}

In this section we investigate the variation of the noise reduction spectra with continually varying flow speed. This data is obtained by measuring the acoustic pressure as the fan speed is steadily reduced (or increased). Spectra are computed over short time intervals over which the change in flow speed is assumed to be negligible. In the present study, 'blow-down' measurements are conducted over a range of mean jet velocities between 55 to $25 \mathrm{~m} / \mathrm{s}$ for aerofoils with and without LE-serrations.

Figure 4.16 shows the 'blow down' plots for the serrated flat plate and aerofoil at identical serration amplitude and wavelength. Comparison of their respective results (Figure 4.16a and b) show that the lower frequency limit is the same for flat plate as well as aerofoils suggesting that the flat plate captures the essential physics of the noise reduction mechanism achievable by the use of leading serrations. It clearly reveals the existence of a lower frequency limit $f_{3 d B}$, shown as the lower dotted line, below which the serrations produce negligible noise reductions. This lower frequency limit, which was also observed in the flat plate study in Narayanan et al. (2014), is seen to be a linear function of jet velocity. Previous study of this lower frequency limit suggests it occurs when the gust wavelength $U / f$ is a certain fraction $\alpha$ of the serration amplitude $h$. This coefficient $\alpha$ is determined from the gradient of the lower dotted line in figure 4.16 which is consistent with our previous finding of 0.2 . The value of 0.2 is only around optimum wavelength.

In figure 4.16 the approximate frequency at which maximum noise reduction is obtained versus flow speed is shown as the middle dashed line, which also appears to follow a constant Strouhal number dependence of $f L_{0} / U$, where $L_{0}$ is an arbitrary constant. Also shown in figure 4.16 as the upper dotted line is the frequency above which noise reductions are negligible. The noise reduction bandwidth for the flat plate is higher than for the aerofoils due to a lower level of self-noise for the flat plate compared to interaction noise. The difference in noise reductions at lower frequencies where interaction noise is dominant because for the flat plate all the aeroacoustic sources lie in the plane whereas they are distributed in 3D for the 3D aerofoils. Detailed analysis of the flat plate results has been already presented by Narayanan et al. (2014). 


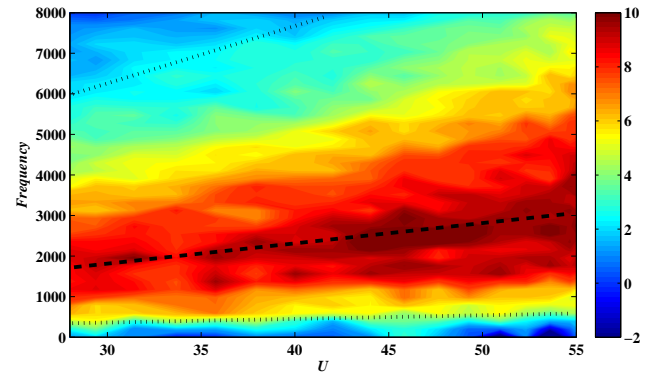

(a) Flat Plate

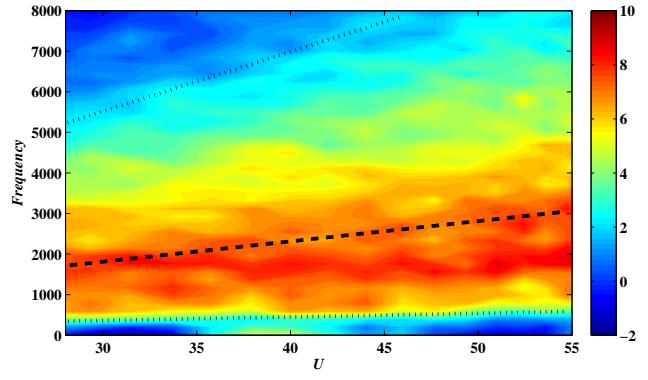

(b) Aerofoil

FIGURE 4.16: Blow down map of $\Delta$ PWL for serration amplitudes $\left(h / c_{0}\right)$ of 0.167 and serration wavelengths $\left(\lambda / \Lambda_{t}\right)$ of 5.33

For completeness, figure 4.17 provides a comparison between the predicted (taken from Chaitanya et al. (2015b)) and measured noise reduction spectra for the flat plate and serrated aerofoils with serration angles close to the optimum. Flat plate and 3D aerofoil noise reductions are compared for a fixed serration amplitude $h / c_{0}=0.167$ and wavelength $\lambda / \Lambda_{t}=5.33$. The measured and predicted spectra seem to be in close agreement and closely follow the empirical curve $\Delta P W L=10 \log _{10}(f h / U)+10$. This relation therefore appears to be widely valid and is supported by numerical predictions that do not reproduce trailingedge noise. This again confirms that the flat plate aerofoils are representative of $3 \mathrm{D}$ aerofoils in order to capture the physics of noise reductions.

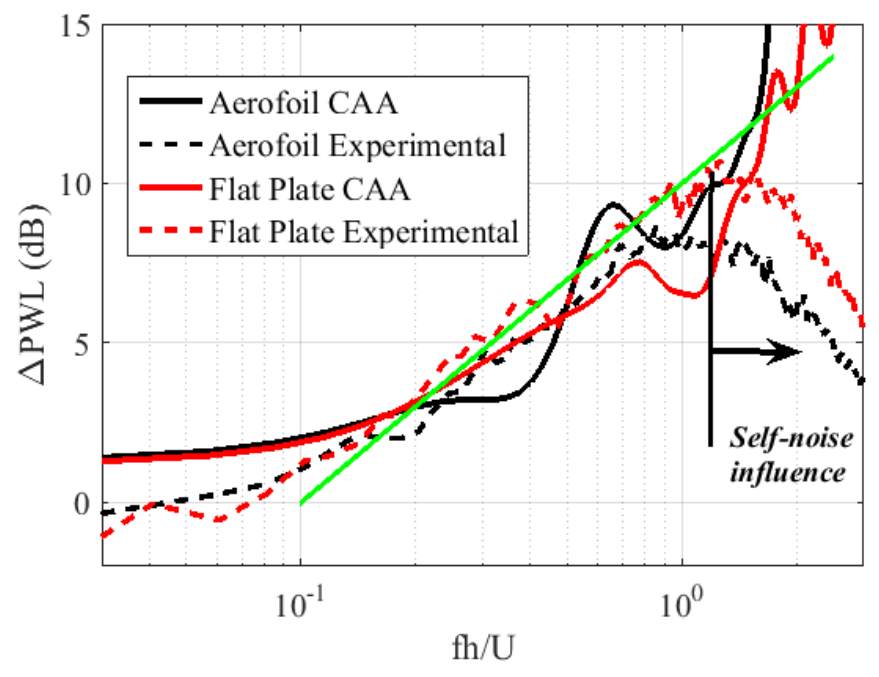

FIGURE 4.17: Comparison of sound power reductions for aerofoil and flat plate of serration amplitude $h / c_{0}=0.167$ and wavelength $\lambda / \Lambda_{t}=3.33$ (taken from Chaitanya et al. (2015b))

\subsubsection{Effect of angle of attack}

Figures 4.18 show the effect of angle of attack on the interaction noise of the baseline and serrated NACA65 aerofoils, which in both cases is negligible. This insensitivity to angle of attack for conventional sharp edge aerofoils is well documented in the literature. Showing 
the same insensitivity for leading serrations demonstrates that the mechanism of noise generation at the leading is similar in both cases and that noise reductions are due to interference across the leading edge.

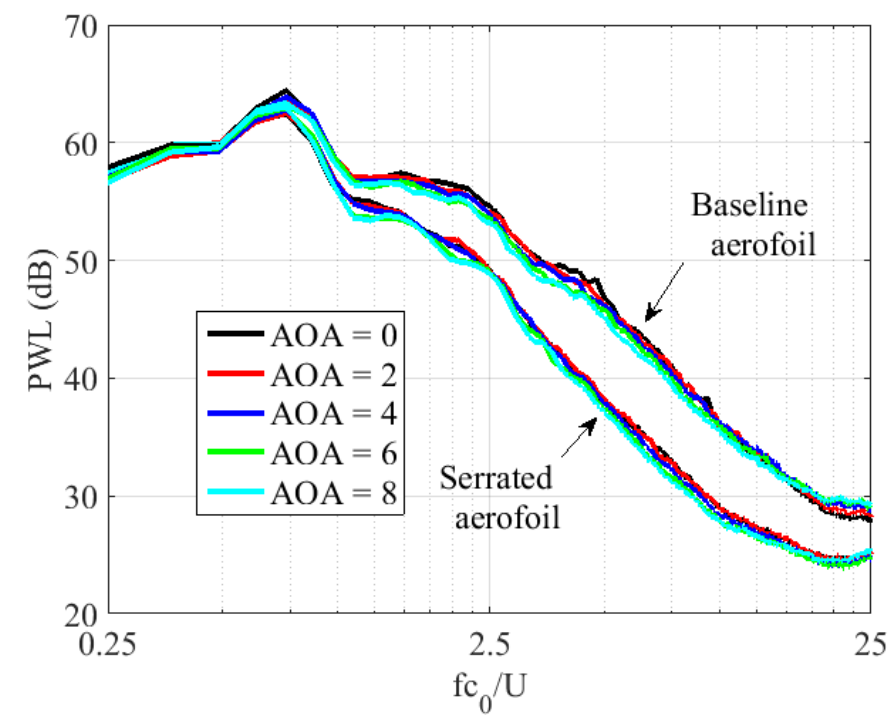

FIGURE 4.18: Power level spectra comparison at various AOA for baseline and serrated aerofoil of amplitude $\left(h / c_{0}\right) 0.167$ and wavelength $\left(\lambda / \Lambda_{t}\right) 2.67$ at Jet velocity $60 \mathrm{~m} / \mathrm{s}$

\subsection{Aerodynamic Measurement}

Previous sections have highlighted the potential effectiveness of leading edge serrations on 3D aerofoils. However, it is imperative that aerodynamic performance is not substantially degraded by their introduction. In this section, the effect of leading edge serrations on the steady aerodynamic performance of aerofoils is investigated experimentally. The lift and drag forces were measured on the NACA65 aerofoil with varying serration amplitudes $\left(h / c_{0}\right)$. The geometric angle of attack varies from $-2.5^{\circ}$ to $10^{\circ}$ but due to jet deflection in the open jet wind tunnel the corresponding effective angle of attack is in the smaller range, $-1^{\circ}$ to $2.8^{\circ}$. Aerodynamics measurements were performed in the same open jet wind-tunnel as the PIV and acoustic measurements to ensure consistency.

Figure 5.14a shows the lift coefficient for the baseline and serrated aerofoil of wavelength $\left(\lambda / \Lambda_{t}\right) 2.67$ for three different serration amplitudes $h / c_{0}$ of $0.066,0.1$ and 0.167 . Whilst the gradient of lift coefficient versus angle of attack remains unchanged by the introduction of serrations (Gradient $\approx 6.2$ /radian), levels are consistently smaller by between 0.01 and 0.05 compared to the baseline case. Thus, whilst increasing the serration amplitude increases noise effectiveness, the corresponding lift performance degrades. The corresponding variation in drag coefficient is shown in figure $5.14 \mathrm{~b}$ which is observed to increase by between 0.001 and 0.005 compared to the baseline case as serration amplitude is increased. Further examination of the aerodynamic performance of the serrated aerofoil is explored in section 4.6 using PIV measurements. It is worth noting that the absolute values of lift and drag coefficient are lower than actual values available in literature (Liu et al. (2015)). This ambiguity can be due to end effects of the setup in open jet wind tunnel. However, the purpose 


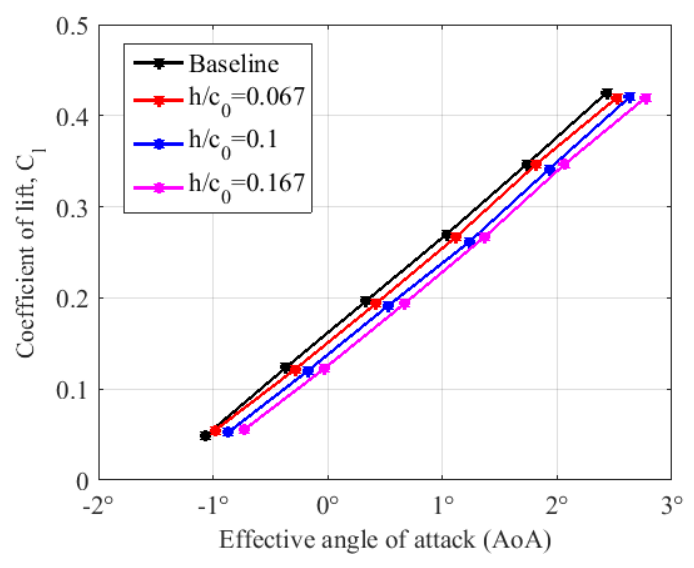

(a) Lift coefficient

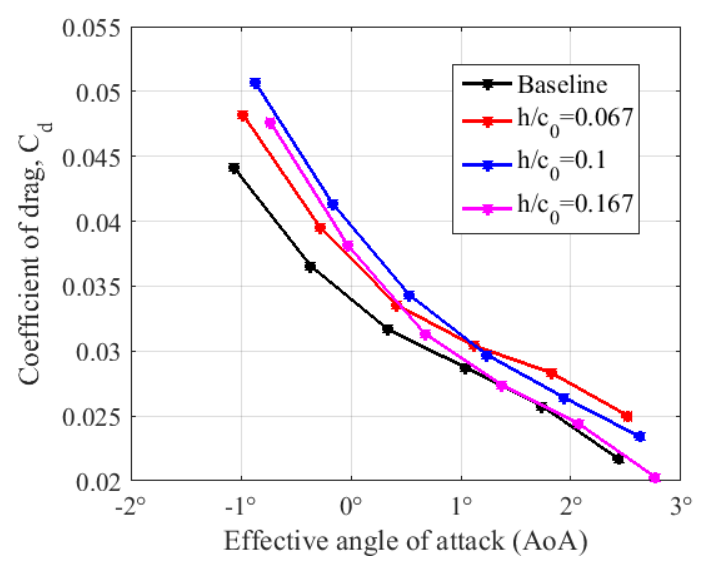

(b) Drag coefficient

FIGURE 4.19: Aerodynamic evaluation of serrated aerofoils $\left(\lambda / c_{0}=0.067\right)$ at the jet velocity $U=60 \mathrm{~m} / \mathrm{s}$

of this section is to demonstrate the aerodynamic penalty due to leading edge serrations at very low effective angles of attack.

\subsection{Flow measurement}

\subsubsection{Mean flow}

This section examines in greater detail the effect of leading edge serrations on the flow behavior around the aerofoil by the use of PIV measurements. Mean velocity contour with streamlines superimposed are presented in figure $4.20 \mathrm{a}-\mathrm{d}$ for a free-stream velocity of $20 \mathrm{~m} / \mathrm{s}$. The inflow turbulence intensity is $2.5 \%$ and the transverse length scale of incoming turbulence is $3.75 \mathrm{~mm}$. The results reveal the flow across two transverse planes aligned with the serration peak and valley at the two geometric angles of attack of $0^{\circ}$ and $10^{\circ}$ (Effective angles of attacks are $0^{\circ}$ and $2.8^{\circ}$ respectively). Also shown in figure $4.20 \mathrm{e}-\mathrm{f}$ are the flow velocity contours for the baseline NACA65 aerofoil. Note that the white region corresponds to the locations where the LASER was unable to illuminate. In both valley and peak planes the mean flow is attached at both angles of attacks even though there are large mean velocity gradients.

Figures $4.20 \mathrm{e}, \mathrm{f}$ show the variation of axial flow velocity around the leading edge of the baseline aerofoil. They show the presence of a significant stagnation region at the leading edge for both angles of attack. The stagnation region around the peak of the serrated aerofoil is considerably weaker (figures $4.20 \mathrm{a}-\mathrm{d}$ ) which is most likely because the flow streamlines can be diverted radially from the peak to the trough. In the plane of the serration valley, however, the streamlines clearly continue in the gap between the serrations. However, since the valley cannot be illuminated and therefore the extent of the stagnation region around the valley is not accessible.

The mean flow component normal to the measurement axis exhibits similar behaviour to the axial flow, as in figure 4.21 which shows a zoomed-in view in the vicinity of the leading edge for the angle of attack of $2.8^{\circ}$. Flow deviation around the peak can be seen to be substantially weaker than in the baseline case. However, streamlines emerging from the valley are 


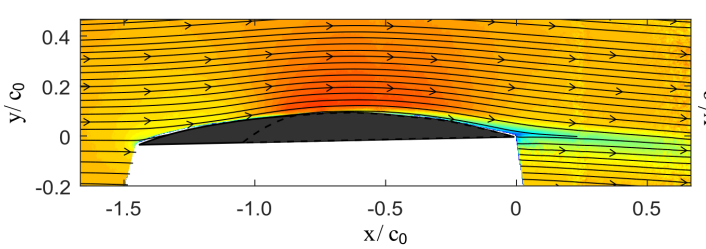

a) Plane of serration peak $\left(\mathrm{AoA}=0^{\circ}\right)$

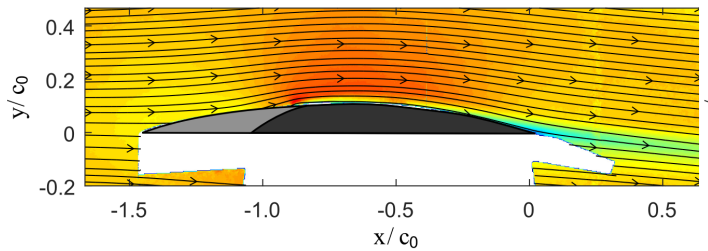

c) Plane of serration valley $\left(\mathrm{AoA}=0^{\circ}\right)$

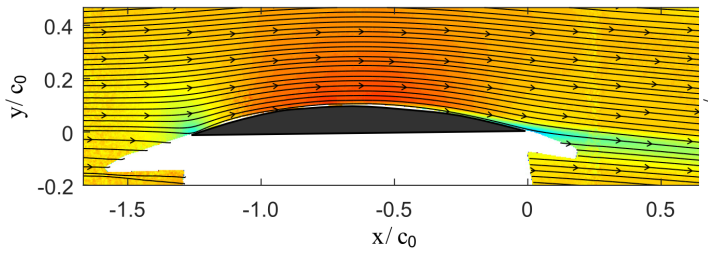

e) Baseline aerofoil $\left(\mathrm{AoA}=0^{\circ}\right)$

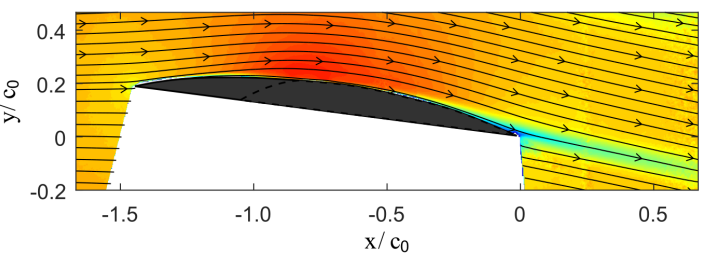

b) Plane of serration peak $\left(\mathrm{AoA}=2.8^{\circ}\right)$

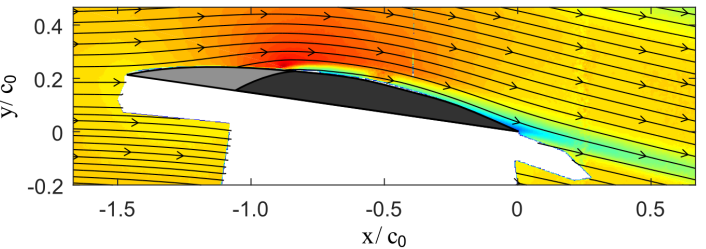

d) Plane of serration valley $\left(\mathrm{AoA}=2.8^{\circ}\right)$

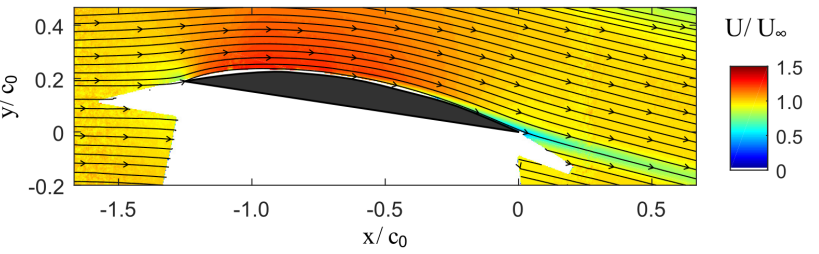

f) Baseline aerofoil $\left(\mathrm{AoA}=2.8^{\circ}\right)$

FIGURE 4.20: Mean velocity maps for serrated and baseline aerofoil for effective angle of attack of $0^{\circ}$ and $2.8^{\circ}$. The aerofoil cross-sections are illustrated to scale and regions where the experimental geometry obscured the measurements are left blank.

observed to undergo stronger deviation than for the baseline case, suggesting strong mean velocity gradients. These large velocity gradients could be a source of turbulence generation and hence a source of additional noise. However, more work is needed through the use, for example, LES or PIV measurement in between the valley to quantify the importance of this phenomenon.

\subsubsection{Effect on leading edge serrations on boundary layer development}

In section 4.4.6 it was observed that the introduction of leading edge serrations caused a significant reduction in trailing edge noise suggesting that boundary layer development was affected. This hypothesis is confirmed in figure 4.22 which compares the PIV measurements of stream-wise boundary layer development over the suction surface in the two planes of

(a) Plane of serration peak

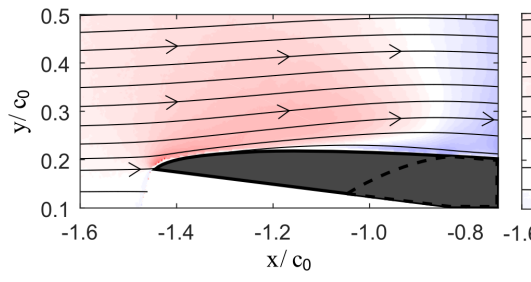

(b) Plane of serration root

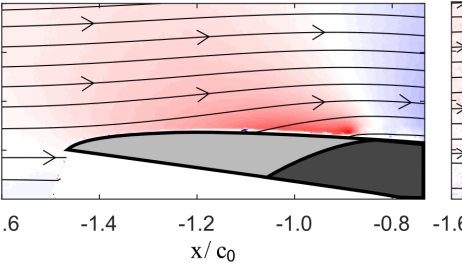

(c) Baseline aerofoil

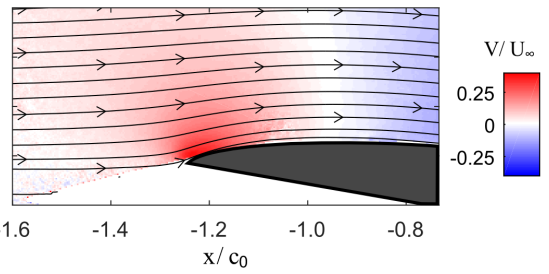

FIGURE 4.21: Maps of the vertical component of the mean velocity at the leading edge of the serrated and baseline aerofoils at an angle of attack of $2.8^{\circ}$. 
the serrated aerofoil with the baseline case at $2.8^{\circ}$ angle of attack. In the plane of the valley the boundary layer is seen to be significantly thicker than in both the peak plane and baseline cases. This is due to delayed vertical deflection of the flow at the valley of the serrations causing a large input of momentum into the boundary layer, which initially causes a substantial thickening of the boundary layer in this plane. Close to the leading edge (figures $4.22 \mathrm{a}, \mathrm{b})$ the profiles at the peak plane and baseline cases are similar. As the boundary layer develops towards the trailing edge their profiles in both planes become similar due to turbulent mixing resulting in a significantly thicker boundary layer at the trailing edge compared to the baseline case.

Figure 4.23 shows profiles of the rms velocity fluctuations along the upper surface of the aerofoil. Just downstream of the serration valley the velocity fluctuations are much higher in the plane of the valleys compared to the baseline case, while they are reduced in the plane of the peak. This is consistent with our earlier observation that velocity fluctuations are reduced at the leading edge peak and localized in the valleys of the serrations as a result of the stagnation region being spread across a wider region compared to the baseline aerofoil. These higher fluctuations in the plane of the valley are also consistent with the thicker boundary layer that develops in this plane. In all the boundary layer profiles shown in this section, at position $\mathrm{y}=0$ (location on the aerofoil surface), the velocity is assumed to be zero, as practically $\mathrm{y}=0$ can not be measured.

The mean velocity profiles at the trailing edge plotted in figure $4.22 \mathrm{e}$ and the rms velocity profile plotted in figure 4.23e are consistent with reduced self-noise radiation plotted in figure 4.13b. The work of Blake (1970) and more recently by Stalnov et al. (2015),has shown that the surface pressure spectrum close to the trailing edge, and hence the far field radiation, is partly determined by the product of mean shear profile $(d U / d y)^{2}$ and the mean square velocity profile integrated through the boundary layer. Whilst the rms velocity profile is only slightly increased at the trailing edge compared to the baseline case, the mean shear gradients are significantly reduced, particularly close to the wall, which is responsible for self-noise generation at high frequencies (since high frequencies are generated by small eddies convecting close to the wall).

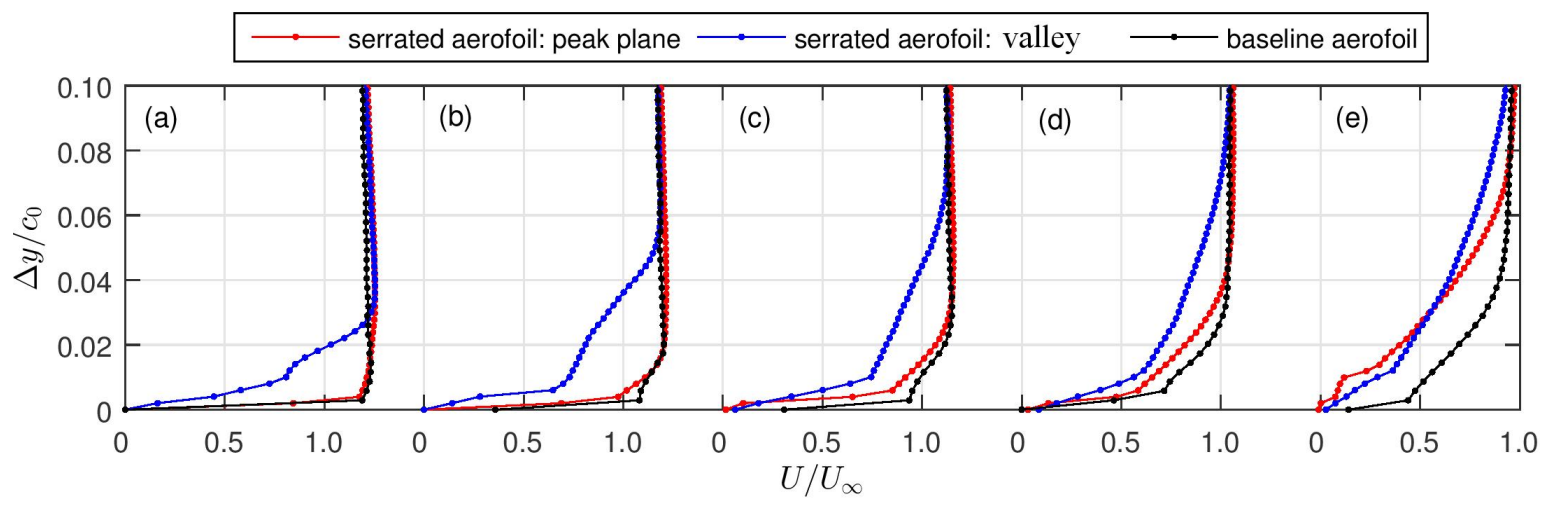

FIGURE 4.22: Development of the boundary layer over the suction side of the aerofoil at locations of distance of (a) $-0.8 c_{0}$, (b) $-0.6 c_{0}$, (c) $-0.4 c_{0}$, (d) $-0.2 c_{0}$, and (e) 0 from the trailing edge of the aerofoil. 


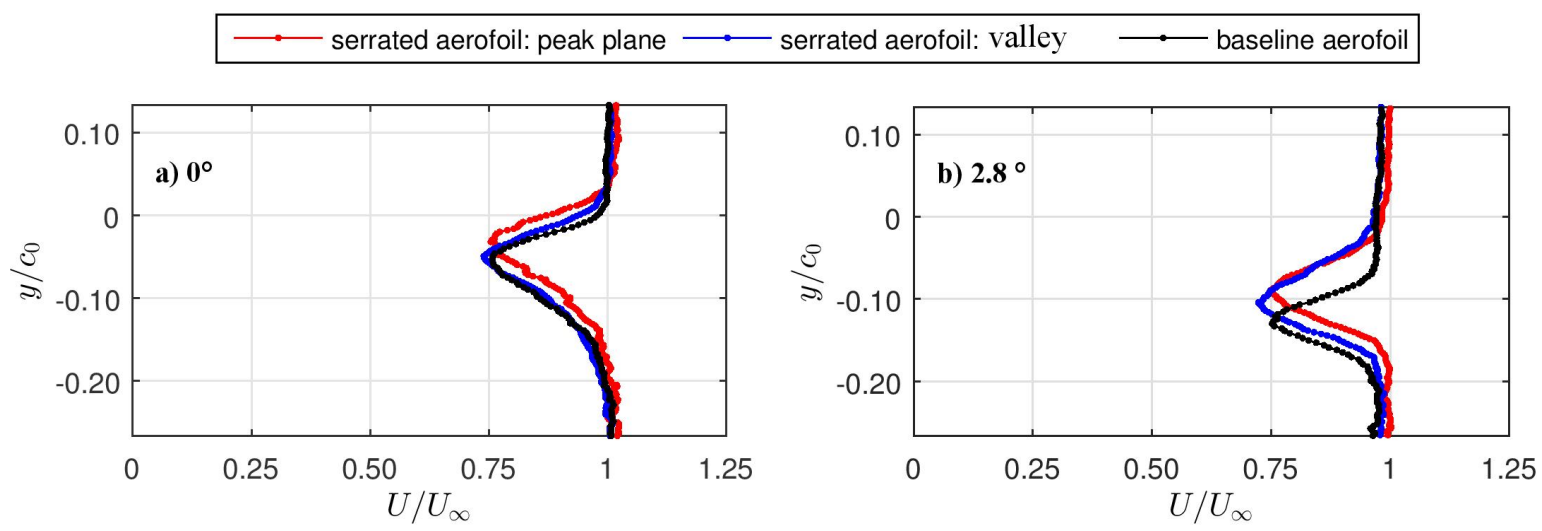

FIGURE 4.24: Stream-wise velocity profiles of the wake measured $70 \mathrm{~mm}$ downstream of the trailing edge for effective angles of attack of (a) $0^{\circ}$ and

(b) $2.8^{\circ}$ where the geometric angles of attacks are $0^{\circ}$ and $10^{\circ}$ respectively.

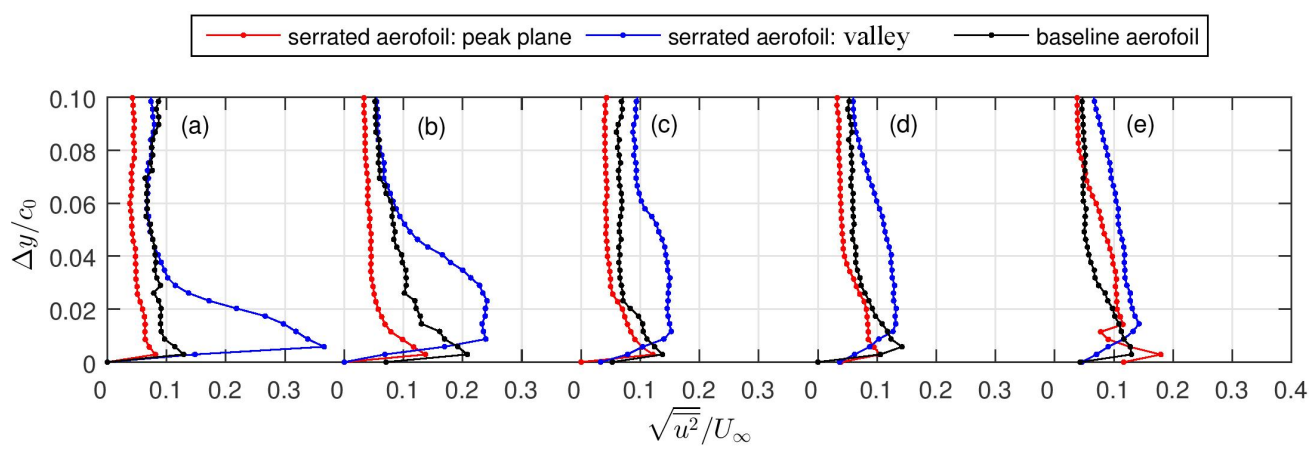

FIGURE 4.23: Profiles of the root-mean-square stream-wise velocity fluctuations along the suction side of the aerofoil at locations of distance of (a) $-0.8 c_{0}$, (b) $-0.6 c_{0},(\mathrm{c})-0.4 c_{0},(\mathrm{~d})-0.2 c_{0}$, and (e) 0 from the trailing edge of the aerofoil.

\subsubsection{Wake characteristics}

In Section 4.6.2 it was observed that the serrated aerofoil has a thicker boundary layer compared with the baseline aerofoil. This implies that the velocity gradient near the wall is lower. The skin friction drag along the upper surface could therefore be decreased. However, a thicker boundary layer could also lead to a broader wake, thereby increasing the pressure drag. Figure 4.24 shows the stream-wise velocity profile measured downstream of the trailing edge through the wake. Wider wake profiles are observed in the case of the serrated aerofoils compared to the baseline aerofoil suggesting an increase in drag. These findings are consistent with the drag measurements obtained directly and presented in section 4.5 .

The lift force produced by the aerofoils may also be analyzed by considering the downward displacement of the wake with respect to $y=0$ as an indicator of flow turning. Figure 4.24 indicates that the wake of the serrated aerofoil is displaced by roughly $20 \%$ less than the baseline case indicating lower lift at both angles of attack. This is consistent with the reported decrease in lift observed by Hansen et al. (2011); Johari et al. (2007) and lift measurements presented in figure 5.14. 


\subsection{Summary}

Consistent with the results from previous work, single-wavelength leading edge serrations have been found to provide substantial noise reductions over a range of frequencies and flow speeds. Based on careful measurements of the sound power reduction for a range of serration amplitudes, wavelengths and two different turbulence length-scales, this chapter has derived simple scaling laws with which to understand and predict the noise reduction for arbitrary serration amplitude and wavelength. The most useful finding of this thesis is the existence of an optimum serration wavelength $\lambda_{o}$ at which maximum sound power reductions occur. The findings above allow the optimum single-wavelength serration to be designed for any arbitrary frequency range and integral length scale. This optimum wavelength has been shown to roughly equal four times the integral length scale $\Lambda_{t}$. At the optimum wavelength:

1. The compact sources at adjacent valleys are excited incoherently

2. A geometric similarity condition is observed in which noise reductions are a function of the ratio of serration amplitude $h$ to gust wavelength $U / f$ at frequencies up until the frequency at which self-noise starts to dominate. This ratio corresponds to the Strouhal number $S t_{h}=f h / U$.

3. The ratio of the sound power produced by the serrated leading edge to the sharp edge (baseline) case is found to be inversely proportional to $S t_{h}$ for $S t_{h} \geq 0.2$. This inverse Strouhal dependence observed at the optimum serration wavelength provides insight into the characteristics of the noise generation mechanism. Following the work of Kim et al. (2016), who show that the noise sources on a serrated leading edge are acoustically compact, concentrated at the valleys, and are roughly the same level as on a straight edge. The inverse $S t_{h}$ dependence suggests that the 'length' of these sources along the leading edge must scale linearly with the hydrodynamic wavelength $U / f$.

4. The rate of change of sound power per valley is precisely equal to the number of valleys $N_{r}$ itself.

A very simple, idealised model is proposed to explain the precise variation in sound power reduction with $S t_{h}$. By assuming that noise reductions are due to variations in the phase of the serrated leading edge, and that these match the phase variation due to the gust along the leading edge, a simple function is derived whose minima match very well the measured variation in sound power reduction versus $S t_{h}$. It is emphasise that the proposed model doesn't provide a complete description of the noise reduction mechanism but merely to provide a simple theoretical framework in which to explain the general behaviour.

Introducing serrations onto the aerofoil leading edge has also been shown to produce a significant reduction in trailing edge noise of up to $3 \mathrm{~dB}$. The serration valleys have been shown through PIV measurements to produce a local thickening of the boundary layer at the leading edge, resulting in an overall thicker boundary layer at the trailing edge which is reasonably uniform across the span. At this trailing edge region, mean shear gradients were found to diminish, particularly near the wall, and the rms velocity fluctuation remains similar to the levels for the sharp baseline case. The classical work of Blake (1970) predicts that a reduction in mean shear gradients will result in a reduction in surface pressure spectra and hence far field noise.

The effect on aerodynamic performance of leading edge serrations has been investigated through direct measurement of the lift and drag and qualitatively through PIV measurements on the same wind tunnel used to perform the noise measurements. The introduction 
of leading edge serrations has been demonstrated to cause a reduction in lift coefficient by between 0.01 and 0.05 approximately and an increase in drag coefficient of 0.001 and 0.005 .

In additional to its structural importance, the purpose of OGV's in turbofan engines are to provide maximum aerodynamic performance by making the flow completely axial with minimum pressure loss coefficient across the them. Leading edge serrations has demonstrated high levels of noise reductions with little penalty on aerodynamics performance. At realistic mean flow velocities in turbofan engines (especially approach conditions), the hydrodynamic gust wavelengths $U / f$ are very large and hence we need to have significantly longer serration amplitudes in order to achieve atleast $3 \mathrm{~dB}$ of noise reductions. This results in deterioration in it's aerodynamic performance. The next chapters in the thesis will be focusing on the maximizing the acoustic performance for a fixed peak-to-root serration amplitude so that aerodynamic performance is not compromised. 



\title{
Chapter 5
}

\section{Noise reductions through double-wavelength leading edge serrations; a new control concept}

\author{
Contents

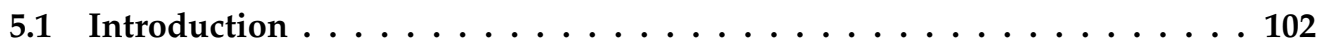 \\ 5.2 Double-wavelength leading edge serrations . . . . . . . . . . . . 103 \\ 5.3 Flat plates leading edge serrations . . . . . . . . . . . . . . . . 104 \\ 5.4 Comparison of noise reductions due to double-wavelength and single- \\ wavelength serrations . . . . . . . . . . . . . . . 105 \\ 5.5 Sensitivity on noise reductions due to $h_{r}$ and $\lambda_{r} \ldots \ldots \ldots \ldots$ \\ 5.5.1 Influence on noise reductions due to $h_{r} \ldots \ldots \ldots \ldots$ \\ 5.5 .2 Influence on noise reductions due to $\lambda_{r} \ldots \ldots \ldots \ldots \ldots$ \\ 5.6 Simple analytical model for additional noise reductions . . . . . . . . 110 \\ 5.7 Self-similarity of double-wavelength serrations . . . . . . . . . . . 113 \\ 5.8 Predicted dependence of $\Delta \mathbf{P W L}_{I}$ on $h_{r}$ and $\lambda_{r}$ on noise reductions $\ldots 114$ \\ 5.8.1 Noise reductions at the tuned frequency $\omega_{0} \ldots \ldots \ldots \ldots$ \\ 5.8 .2 Optimum condition $\left(\omega_{0}=\omega_{\Lambda}\right) \ldots \ldots \ldots \ldots \ldots$ \\ 5.8 .3 Noise reduction frequency bandwidth . . . . . . . . . . . . . . 116 \\ 5.9 Sound Power Spectra comparison for 3-D aerofoils . . . . . . . . . . 118 \\ 5.10 Aerodynamic performance . . . . . . . . . . . . . . . . . . 119

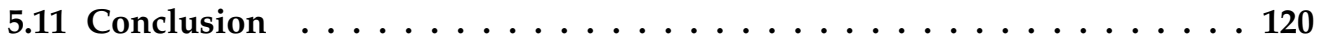

The aeroacoustic and aerodynamic performance of single-wavelength sinusoidal serrations has been studied extensively in the Chapter 4, where simple scaling laws were identified under some conditions. Nearly all of the previous work available in the literature has focused on sinusoidal leading serration geometries. It was observed previously in Chapter 4 that as serration amplitude is increased, aerodynamic performance degrades. The goal of the present chapter is to maximize the acoustic performance for a fixed serration amplitude, thereby achieving a better balance between acoustic performance and aerodynamic penalty. This chapter presents alternative leading-edge serration design based on a new noise control principle for the reduction of aerofoil leading edge noise that provide substantially greater noise reductions than single-wavelength serrations. The new leading edge serration profiles simply comprise the superposition of two single-wavelength components of different wavelength, amplitude and phase with the objective of forming two roots that are sufficiently close together and separated in the streamwise direction. Compact sources located at these root locations then interfere leading to less efficient radiation than single-wavelength geometries. 


\subsection{Introduction}

The single-wavelength profiles have been shown to involve two dominant noise reduction mechanisms. One is due to a source cut-off effect arising from the obliqueness of the inclined leading edge (Clair et al., 2013; Kim et al., 2016). The second is due to interference between the sources along the serration leading edge. However, one of the main findings of Kim et al. (2016) is that the source at the root is dominant compared to the sources at the tip and the sources along the oblique edge and is similar in strength to the straight leading edge. The reason for this behavior has been investigated by Turner et al. (2016). They showed that the root is the dominant noise source due to the presence of a secondary horseshoe-like vortex system generated by the serrated leading edge, which alters the upstream velocity field, thereby enhancing the source strength at the serration root.

Chapter 4 on single-wavelength serrations has demonstrated the existence of an optimum wavelength $\lambda_{0}$ which is a roughly four times the transverse integral length scale $\Lambda_{t}$ of the incoming turbulent flow. At this optimum wavelength, it was shown the reduction in sound power level is given by $\Delta \mathrm{PWL}(f)=10 \log _{10}(f h / U)+10$, where $h$ is the serration amplitude, $U$ is the velocity. This expression represents the upper limit on the possible reductions in sound power that can be achieved through the use of single-wavelength serrations at any particular frequency $f$ and is determined only by the non-dimensional frequency $f h / U$.

Chapter 4 of the thesis has outlined the reason for the existence of an optimum wavelength $\lambda_{0}$ for single-wavelength serrations, which is related to the turbulence integral length scale $\Lambda_{t}$ by 4 . It wass argued that this value corresponds to the maximum spanwise separation distance between adjacent roots at which the root sources just become incoherent. At smaller separation distances, therefore, adjacent roots interfere constructively, leading to a deterioration in the noise reduction performance. In the serration profile proposed here, therefore, the distance between roots is required to be less than this value in order to exploit the effects of interference between adjacent roots.

In this chapter we propose an alternative leading edge geometry that is able to provide significantly enhanced sound power reduction compared to the maximum sound power reduction that can be obtained from single-wavelength serrations. A new control principle is proposed in which different source components at the leading edge are arranged to interfere destructively. The new leading edge profile simply comprises the superposition of two single-wavelength components of roughly the same amplitude but with one wavelength being twice the second and shifted in phase. The resulting profile then comprises adjacent (nearly-identical) roots which are separated in the streamwise direction. Upon interaction with a turbulent flow, therefore, near-identical radiation is generated at each root but with a phase difference corresponding to the time taken for the turbulent flow to convect between the two roots. Maximum noise reductions are obtained at the frequency at which the two roots radiate $180^{\circ}$ out of phase. Preliminary experimental results confirming this new control concept was originally proposed by Chaitanya et al. (2016) and validated numerically by the numerical simulations of Turner et al. (2016).

This chapter presents a detailed experimental investigation into the effectiveness of this double-wavelength concept on flat plates in a turbulent stream. The sensitivity of noise reductions to the stream-wise and span-wise separation distance between adjacent roots is investigated in sections 5.5.1 and 5.5.2. A simple model is proposed in section 5.6 to predict the reduction in sound power versus frequency and provide physical insight into the influence of separation distance between the roots, which is discussed in section 5.8. Finally the optimum design is implemented on a $10 \%$ thick three-dimensional (3-D) aerofoils. The 
noise reduction spectra, which are presented in section 5.9, is shown to be almost identical to that obtained on the flat plate.

\subsection{Double-wavelength leading edge serrations}

The double-wavelength profile under investigation here is defined as the sum of two singlewavelength components of amplitudes $h_{1}$ and $h_{2}$, and wavelength $\lambda_{1}$ and $\lambda_{2}$ with phase difference $\phi$. The chord length $c(r)$ at any radial position $r$ is therefore given by,

$$
c(r)=c_{0}+h_{1} \sin \left(2 \pi r / \lambda_{1}\right)+h_{2} \sin \left(2 \pi r / \lambda_{2}+\phi\right)
$$

where $c_{0}$ is the mean chord.

Adding two sinusoidal profiles has the desired property of forming two adjacent roots separated in the streamwise direction by $h_{r}$ and separated in the spanwise direction by $\lambda_{r}$, giving a total peak-to-root amplitude of $2 h$, as shown in the sketch in figure 5.1. The red dots in this figures represent the compact sources regions at the root locations, which will form the basis of a simple model for predicting the noise reductions in section 5.6. This sketch also shows an upstream turbulent eddy impinging on the leading edge at a flow speed $U$ with transverse integral length-scale $\Lambda_{t}$. Note that no simple relationship exists between the single-wavelength parameters $h_{1,2}, \lambda_{1,2}$, the phase difference $\phi$ and the distances $h_{r}$ and $\lambda_{r}$, which we shall show below are central to the performance of the double wavelength serration. These distances must therefore be obtained by a trial and error process to provide the necessary values identified below for best control performance.

The profile sketched in figure 5.1 is designed to produce near-identical sources at the roots but which differ in phase by $\phi(\omega)=\omega h_{r} / U$ by virtue of being separated in the streamwise direction by a distance $h_{r}$. This profile therefore leads to dipole-like radiation at the frequency $\omega_{0}$, i.e., $\phi\left(\omega_{0}\right)=\pi$, which we shall now refer to as the tuned frequency, for which the sources are exactly $180^{\circ}$ out of phase, given by

$$
\omega_{0}=\pi U / h_{r}
$$

Note also that based on the principle of destructive interference, additional peaks in the noise reduction spectra are also predicted, occurring at the odd harmonics of $\omega_{0}$ corresponding to $\omega_{0, n}=(2 n-1) \pi U / h_{r}$, provided that the sources are sufficiently coherent at these higher frequencies.

Overall sound power reductions provided by these double-wavelength profiles therefore comprises the sum of two contributions. The first arises from the reduction in source strength along the leading edge, as shown by Kim et al. (2016) for single-wavelength profiles, which we shall denote as $\Delta \mathrm{PWL}_{S}$. The second arises from interference between adjacent root sources, which we shall denote as $\Delta \mathrm{PWL}_{I}$. The overall sound power reduction may therefore be written as,

$$
\Delta \operatorname{PWL}(\omega)=\Delta \mathrm{PWL}_{S}(\omega)+\Delta \mathrm{PWL}_{I}(\omega)
$$




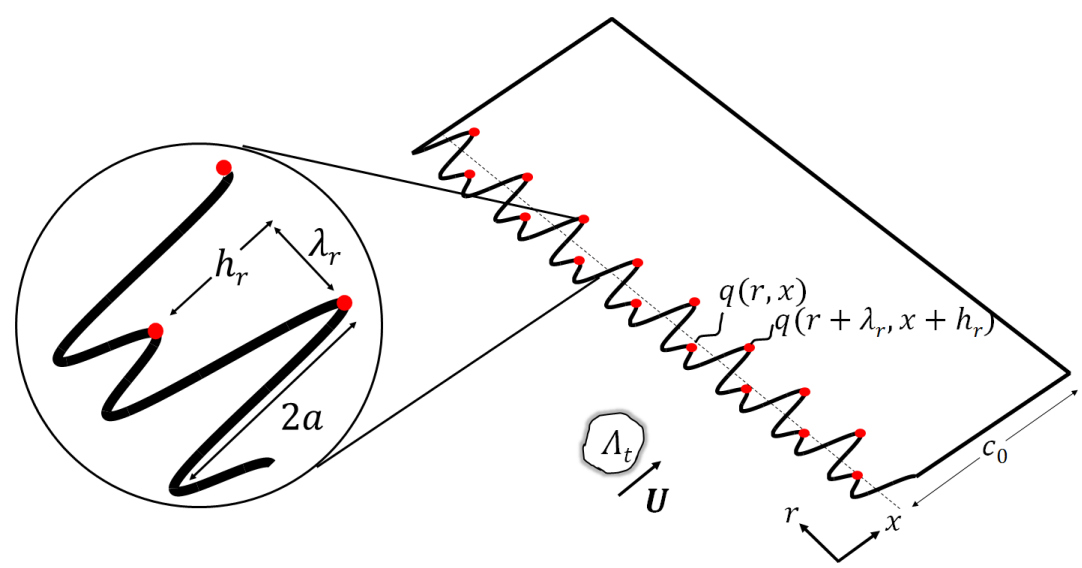

FIGURE 5.1: A typical double-wavelength serration profile formed from the summation of two single-wavelength profiles to form adjacent roots that are separated in the spanwise direction by $\lambda_{r}$ and by $h_{r}$ in the streamwise direction.

Chaitanya et al. (2017) has identified the maximum possible sound power reduction that can be obtained from single-wavelength serrations arising solely from a reduction in the source strength, given by,

$$
\Delta \mathrm{PWL}_{S}(f h / U)=10 \log _{10}(f h / U)+10,
$$

and is only a function of the non-dimensional frequency $f h / U$, where $h$ is half the peakto-root amplitude identified in figure 5.1. The additional noise reduction due to doublewavelength serrations arising from interference between adjacent roots is therefore quantified by the interference term $\Delta \mathrm{PWL}_{I}(\omega)$, which we shall be investigating in detail in Section 5.6. We now present the experimental data confirming the proposed noise reduction mechanism of double-wavelength serrations.

\subsection{Flat plates leading edge serrations}

For economy and ease of manufacture, a parametric study into the effect of $h_{r}$ and $\lambda_{r}$ on noise reductions was performed on flat plates situated within a turbulent flow. A double wavelength design arising from this study was then applied to a 3-D aerofoil of $10 \%$ thickness, which we show in section 5.9 has an almost identical noise reduction spectra to the flat plate case.

The flat plate with a mean chord $\left(c_{0}\right)$ of $150 \mathrm{~mm}$ and span of $450 \mathrm{~mm}$ were constructed by joining together two $1 \mathrm{~mm}$ thick metallic sheets to allow serrated flat plate inserts $2 \mathrm{~mm}$ thick to be inserted between them. All corners were rounded and the trailing edge sharpened to eliminate vortex shedding noise. Further details of this flat plate construction can be found in Narayanan et al. (2015).

A total of 11 different flat plate serrations of varying amplitude, phase and wavelength were investigated to explore the sensitivity on noise reductions to these parameter variations. Note that in this chapter values of $\lambda_{r}$ and $h_{r}$ are presented as quantities normalized on the mean chord $c_{0}$ although there is no evidence to suggest that this is a meaningful parameter 


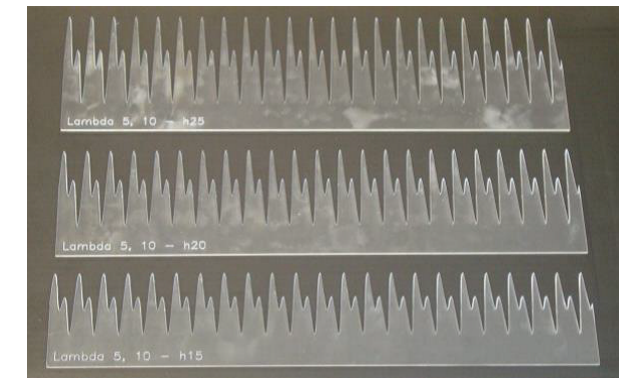

(a) $\lambda_{1} / c_{0}=0.033 \& \lambda_{2} / c_{0}=0.067, h / c_{0}$ $0.1,0.133,0.167$.

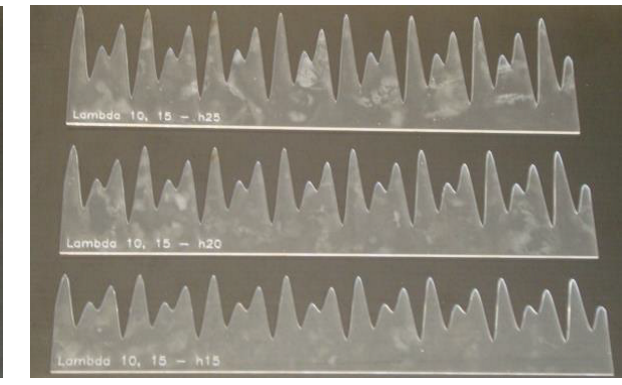

(b) $\lambda_{1} / c_{0}=0.067 \& \lambda_{2} / c_{0}=0.1, h / c_{0}=$ $0.1,0.133,0.167$.

FIGURE 5.2: A typical flat plate double-wavelength serration inserts.

in determining noise reductions. Figure 5.2 shows photographs of six typical profiles, cut from $2 \mathrm{~mm}$ acrylic sheet, investigated in this chapter.

\subsection{Comparison of noise reductions due to double-wavelength and single-wavelength serrations}

Figure 5.3 is a typical sound power reduction spectra plotted against non-dimensional frequency $\omega / \omega_{0}$ obtained for a double-wavelength profile comprising the sum of two singlewavelength serrations with wavelengths of $\lambda_{1} / c_{0}=0.033$ and $\lambda_{2}=2 \lambda_{1}$ with identical serration amplitudes $h_{1} / c_{0}=h_{2} / c_{0}=0.0568$ with zero phase difference $\phi=0$. The flow speed in this case is $U=60 \mathrm{~m} / \mathrm{s}$. This double wavelength profile has a total peak-to-root distance of $2 h / c_{0}=0.2$ and spanwise distance between adjacent roots $\lambda_{r} / \Lambda_{t}$ of 1.17, which we shall show below is a critical parameter in determining the level of noise reductions. A sketch of the profiles are also shown in figure 5.3. Noise reduction spectra due to the doublewavelength serration profile and single-wavelength profiles whose amplitudes were chosen to equal that of the double wavelength profile, $2 h / c_{0}=0.2$, are also shown in this figure. The dashed line in this figure represents the optimum sound power reduction for a singlewavelength serration given by equation 5.4. Note also that at high frequencies, the self-noise radiated from the trailing edge starts to become the dominant noise source causing the noise reduction spectra to deviate from the straight line of equation 5.4, which one would expect based solely on a reduction in source strength.

Simply adding the two single-wavelength components but maintaining overall amplitude can be seen to provide an additional noise reduction compared to single-wavelength serrations of up to $4 \mathrm{~dB}$ in the non-dimensional frequency range of roughly $\omega / \omega_{0}=0.7$ to 1.4. The frequency of maximum noise reductions occurs close to the tuned frequency of $\omega / \omega_{0} \approx 1$, where two adjacent roots are $180^{\circ}$ out of phase. This increase in noise reduction performance therefore provides validation of the control principle of destructive interference between adjacent roots. An important finding in figure 5.3 is that noise reductions are confined to a well-defined frequency bandwidth with no additional noise reductions outside of this bandwidth. A detailed explanation of this behaviour will be provided in section 5.8. 


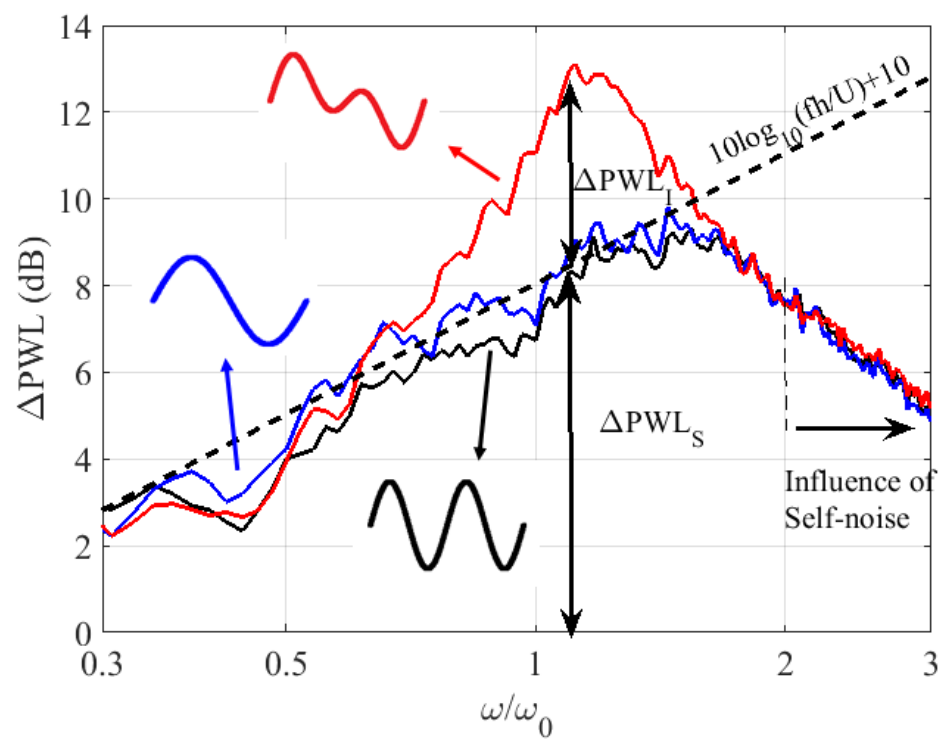

FIGURE 5.3: Acoustic performance of double-wavelength serrations for $\lambda_{1} / c_{0}=0.033 \lambda_{2} / c_{0}=0.067, h / c_{0}=0.1, \phi=0$ and flow velocity $U=60 \mathrm{~m} / \mathrm{s}$. (black line: Single-wavelength $\lambda_{1}$; blue line: Single-wavelength $\lambda_{2}$; red line: Double-wavelength serration).

\subsection{Sensitivity on noise reductions due to $h_{r}$ and $\lambda_{r}$}

In this section the sensitivity of the noise reduction due to double-wavelength serration profiles are compared to the optimum single-wavelength profiles of the same peak-to-root distance due to variations in $h_{r}$ and $\lambda_{r}$.

\subsubsection{Influence on noise reductions due to $h_{r}$}

The noise control principle outlined above suggests that to achieve significant noise reductions at low frequencies requires the streamwise distance between the adjacent roots $h_{r}$ to be as large as possible. The distance between adjacent roots may be altered, either by altering the amplitude of the single-wavelength components $h_{1}$ and $h_{2}$ or by altering the phase between them.

The results of a parametric study are now presented aimed at revealing the sensitivity of noise reductions to overall peak-to-root distance $2 h$, i.e., simply scaling $h_{1}, h_{2}$, but keeping $h_{1}=h_{2}$, to vary $2 h$. Figure 5.4 shows the variation in sound power reductions in $2 h / c_{0}$ between 0.1 and 0.167, whilst keeping $\lambda_{1}$ and $\lambda_{2}$ the same as in Figure 5.2a. It is observed that the peak noise reduction frequency $f h_{r} / U$ varies between 0.5 to 0.6 , even though the root sources are predicted to be $180^{\circ}$ out of phase for $f h_{r} / U=0.5$. This variation in value is because the sources in the vicinity of the root are not located precisely at the root. Clearly, narrower serrations exhibit a much greater variation in stream-wise source location compared to broader serrations. A detailed numerical investigation of this phenomenon is presented by Turner et al. (2016), who demonstrated that destructive interference occurs between upstream root and a point near to the downstream root. This effect is more pronounced for longer serrations as shown in Figure 5.4. 


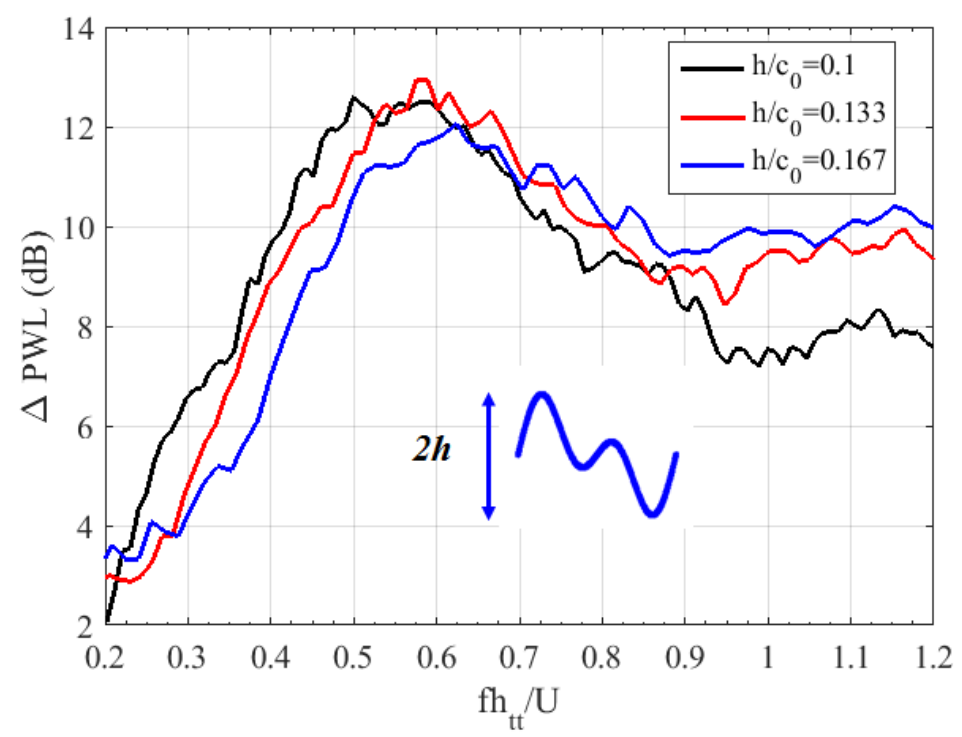

FIGURE 5.4: Acoustic performance of double-wavelength serrations, $\lambda_{1} / c_{0}=$ $0.033 \& \lambda_{2} / c_{0}=0.067, h / c_{0}=0.1,0.133,0.167$.

We now investigate the effect on noise reductions due to varying $h_{r}$ by changing the phase difference $\phi$. Three values of $h_{r}$ were investigated by fixing $h_{1}=h_{2}$ and choosing the phase difference to be $\phi=0, \pi / 4$ and $\pi / 2$. This combination of parameters has the effect of varying $h_{r}$ whilst keeping the peak-to-root amplitude $2 h$ and the spanwise distance between roots $\lambda_{r}$ constant. Note that in this example the spanwise distance $\lambda_{r} / \Lambda_{t}=1.17$ which is considerably less than the value 4.5 , which ensures that adjacent root sources radiate with a high degree of coherence and therefore strongly interfere, as shown in Section 5.5.2. The three noise reduction spectra are plotted in Figure 5.5 against $\omega / \omega_{0}$.

Noise reductions at the peak frequency $\omega / \omega_{0} \approx 1$ diminish as $h_{r}$ increases. A second peak is also observed in the noise reduction spectra at $\omega / \omega_{0} \approx 3$, again confirming the role of interference between adjacent roots as an important noise reduction mechanism. Increasing $h_{r}$ can be seen to have two effects on the noise reduction spectra. One is that the noise reduction reduces at the peak frequency $\omega_{0}$ but increases at the second peak frequency of $\approx 3 \omega_{0}$. The second effect of increasing $h_{r}$ is that the frequency of the first peak steadily increases above $\omega / \omega_{0}=1$ while the second peak moves towards $\omega / \omega_{0}=3$ from above.

One explanation of the shifting peak frequencies is that the sources are not precisely located at the roots, as identified in the numerical simulations of Turner et al. (2016). Another explanation for the diminishing peak noise reductions and shifting frequencies is proposed in Section 5.8.2 and is related to the frequency of maximum coherence length.

\subsubsection{Influence on noise reductions due to $\lambda_{r}$}

In this section we investigate the effect on noise reductions due to variations in the spanwise root-to-root separation distance $\lambda_{r}$. An important requirement for the double-wavelength serration concept to be effective is that the sources between adjacent roots must have a high degree of coherence to be able to interfere destructively. The distance $\lambda_{r}$ is therefore required to be much less than the optimum spanwise distance identified in Chaitanya et al. (2017) for single-wavelength serrations, necessary to ensure that adjacent roots are incoherent over the entire frequency range. We now investigate this dependence explicitly. A parametric 


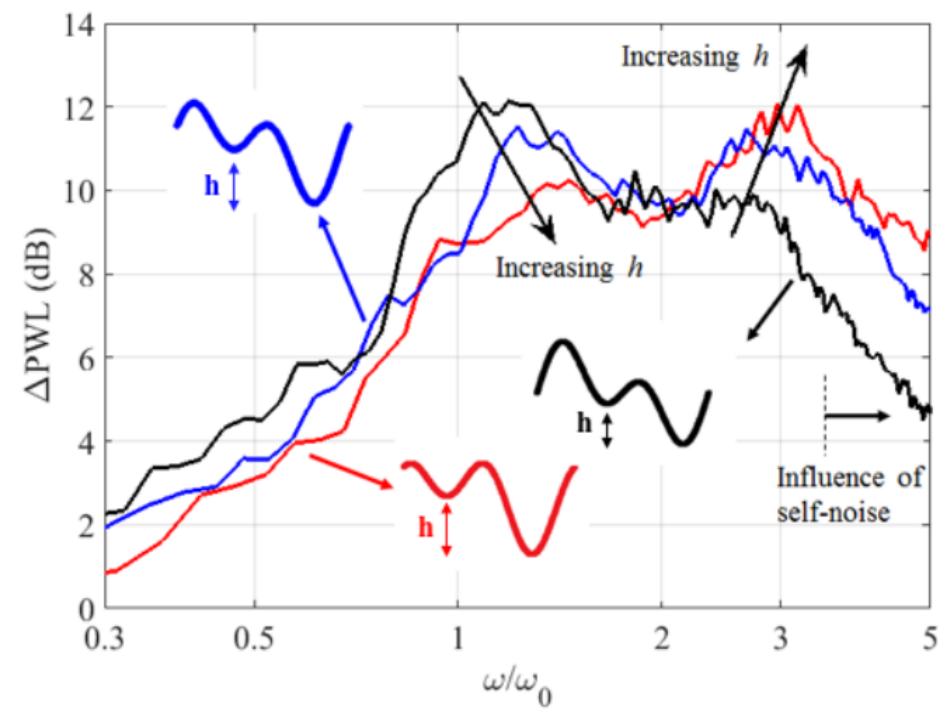

FIGURE 5.5: Acoustic performance of double-wavelength serrations for $\lambda_{1} / c_{0}=0.033 \& \lambda_{2} / c_{0}=0.067, h / c_{0}=0.167, \mathrm{U}=60 \mathrm{~m} / \mathrm{s}$ at varying phase $\phi$ ratios of $0, \pi / 4, \pi / 2$. (black line: $\phi=0$; blue line: $\phi=\pi / 4$; red line: $\phi=\pi / 2$ ).

study is now performed in which only the spanwise distance $\lambda_{r}$ between adjacent roots is varied whilst maintaining constant root-to-root distance $h_{r}$. This was achieved by varying $\lambda_{1}$ but maintaining the ratio between the two wavelengths $\lambda_{1} / \lambda_{2}$ at 0.5 . Figure 5.6 shows the sound power reductions versus $\omega / \omega_{0}$ for 6 different spanwise distances $\lambda_{r} / \Lambda_{t}$ of between 1 to 7 at a fixed peak-to-root amplitude $2 h / c_{0}=0.33$ and a flow speed of $U=60 \mathrm{~m} / \mathrm{s}$. This range of values was chosen to vary either side of the optimum wavelength, $\lambda_{r} / \Lambda_{t}$ of $\approx 4$ for which maximum noise reductions occur for single-wavelength serrations. Also shown in this figure is the line $10 \log _{10}(f h / U)+10$ corresponding to the maximum sound power reduction versus frequency that can be obtained by a single-wavelength serration, which occurs at the optimum wavelength $\lambda_{r}=\lambda_{o}$.

Figure 5.6 can be summarized as follows:

1. Peaks in the sound power reduction spectra at non-dimensional frequencies of approximately 1 are only observed for the three cases in which $\lambda_{r} / \Lambda_{t} \leq 4.5$. In these cases, therefore, adjacent roots strongly interfere and the sound power reductions significantly exceed the fundamental limit $10 \log _{10}(f h / U)+10$ achievable using singlewavelength serrations (Chaitanya et al. (2017)). By contrast, no peaks in the noise reduction spectra can be observed for the three cases in which $\lambda_{r} / \Lambda_{t} \geq 4.5$ for which adjacent root sources are incoherent.

2. Sound power reductions are only achieved over a band of frequencies, which is highly dependent on $\lambda_{r}$. The reason for this behaviour will be explored in section 5.6 below.

3. Sound power reductions generally improve as $\lambda_{r} / \Lambda_{t}$ is reduced below the threshold value of 4.5 suggesting that the level of coherence between the adjacent root sources increases as $\lambda_{r} / \Lambda_{t}$ is reduced and hence the degree of destructive interference is improved.

4. The peak frequency of maximum noise reduction for the cases $\lambda_{r} / \Lambda_{t} \leq 4.5$ increases steadily from the lowest value of 1 to 1.3 at the shortest separation distance $\lambda_{r} / \Lambda_{t} .17$. This behaviour is explored in section 5.8 . 


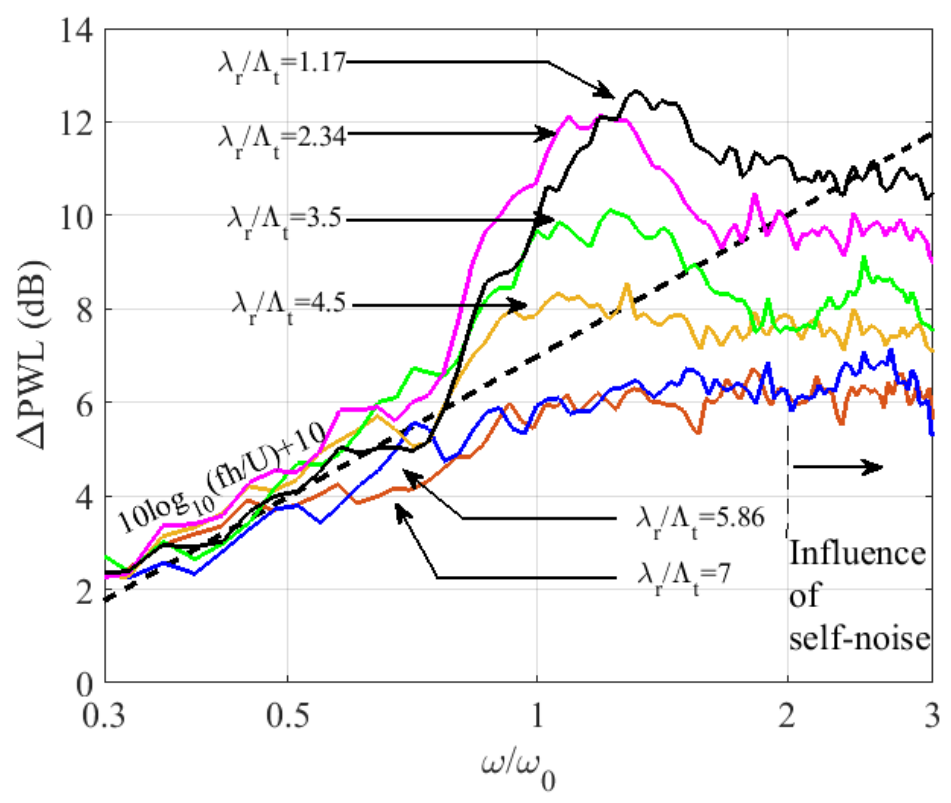

FIGURE 5.6: Influence of serration wavelength on noise reduction at a fixed peak-to-root amplitude $2 h=0.33 \&\left(\lambda_{1} / \lambda_{2}=0.5\right)$ at jet velocity $60 \mathrm{~m} / \mathrm{s}$.

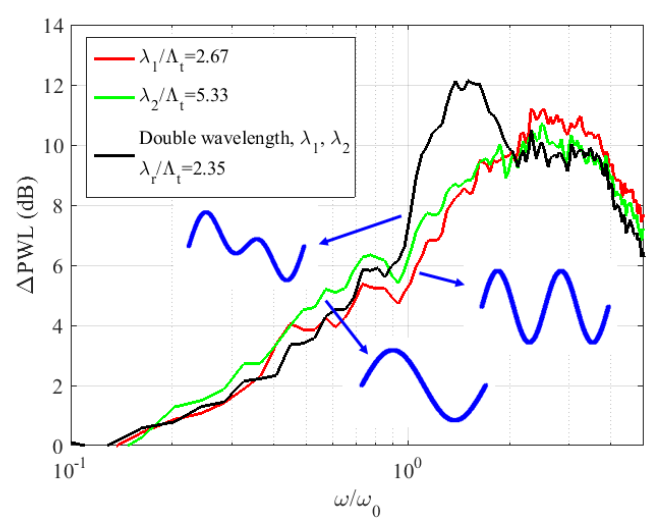

(a) $\lambda_{r} / \Lambda_{t}=2.35$

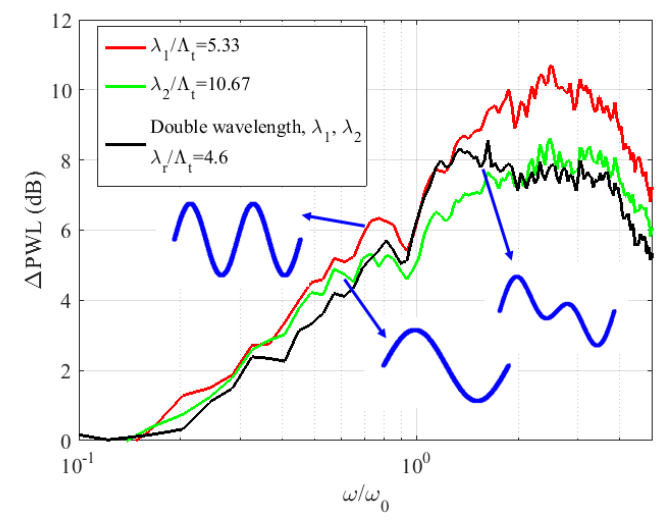

(b) $\lambda_{r} / \Lambda_{t}=4.6$

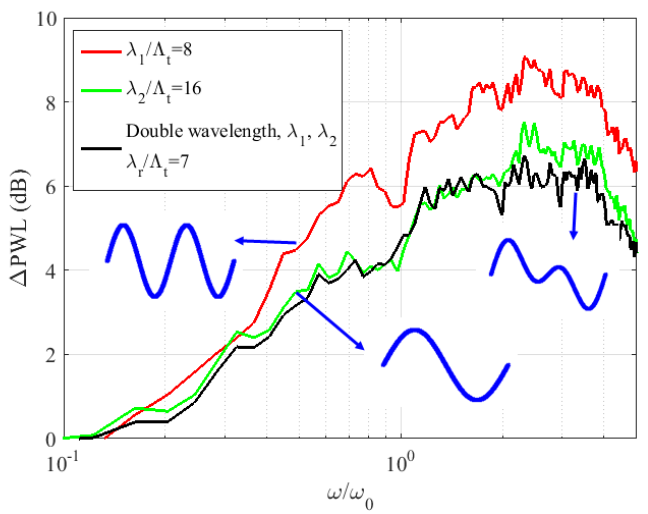

(c) $\lambda_{r} / \Lambda_{t}=7$

FIGURE 5.7: Comparison of Sound power reductions of double wavelength serrations with single wavelength serrations at three different $\lambda_{r} / \Lambda_{t}$ of 2.35, 
To more fully illustrate the behaviour of double-wavelength serrations their noise reduction spectra are now compared to their single wavelength components of the same peak-to-root amplitude. Three cases are considered. One in Figure 5.7a corresponds to $\lambda_{r} / \Lambda_{t}=2.35$ and hence adjacent roots are well within the correlation length of 4 identified in Chapter 4 . The second in Figure 5.7b is for $\lambda_{r} / \Lambda_{t}=4.6$ and corresponds to the case when adjacent roots are on the verge of becoming uncorrelated and Figure 5.7c for $\lambda_{r} / \Lambda_{t}=7$ where adjacent roots are completely uncorrelated. Figure 5.7a clearly shows the additional noise reductions resulting from destructive interference between adjacent roots, leading to better overall noise reductions. However, when adjacent roots are just uncorrelated, noise reductions are equal to the single wavelength serration with the largest wavelength but poorer than than the serration with the smallest wavelength. Finally, when adjacent roots are completed uncorrelated, Figure 5.7c, the noise reduction spectra of the double serration profile tends almost exactly to the single wavelength serration of the largest profile but is significant worse than the smallest wavelength profile.

Thus, the requirement for an effective double wavelength serration profile are contrary to that for single wavelength profiles, whereby the wavelength $\lambda_{r}$ is required to be significantly less than the optimum value of 4 identified for single wavelength profiles.

\subsection{Simple analytical model for additional noise reductions}

In this section a simple analytical model is derived to understand the behaviour of the noise reductions with serration geometry observed experimentally above. In the model we focus on the additional noise reduction $\Delta \mathrm{PWL}_{I}$ due to interference between adjacent root sources.

In this simple model we assume that the root sources have identical source strength but radiate to a far-field observer with a phase difference $\omega h_{r} / U$ arising from the streamwise distance $h_{r}$ between adjacent roots. Consider two compact sources located at spanwise positions $r_{1}$ and $r_{2}=r_{1}+\lambda_{r}$, and streamwise locations $x_{1}$ and $x_{2}=x_{1}+h_{r}$ with source strengths $q\left(r_{1}, x_{1}, \omega\right)$ and $q\left(r_{2}, x_{2}, \omega\right)$ along the span of the aerofoil, as shown in figure 5.1. The path length differences between adjacent roots to a far-field observer can be neglected since they are generally much closer together compared to an acoustic wavelength. The total radiated pressure at any given frequency $\omega$ is therefore proportional to the sum of the source strengths with relative phase difference included,

$$
p(\omega) \propto q\left(r_{1}, x_{1}, \omega\right)+q\left(r_{2}, x_{2}, \omega\right) e^{-i \omega h_{r} / U}
$$

The level of additional noise reduction due to interference between adjacent roots is therefore determined by the degree of coherence between them as turbulent flow convects over the aerofoil. In this chapter, an assumption was made that the turbulence is frozen, i.e., $v(\mathbf{x}, t)=v(\mathbf{x}-\mathbf{U} t)$, where $v$ is the velocity component normal to the aerofoil and $\mathbf{x}=(r, x)$ is any position in the flow. Further making the assumption that the turbulence is homogeneous, the space-time correlation function $R=E\left[v\left(r_{1}, x_{1}, t_{1}\right) v\left(r_{1}+\lambda_{r}, x_{1}+h_{r}, t_{2}\right)\right]$ for the velocity $v$ between the two positions $\left(r_{1}, x_{1}\right)$ and $\left(r_{2}, x_{2}\right)$ is of the form

$$
R\left(r_{1}, x_{1}, r_{1}+\lambda_{r}, x_{1}+h, t_{1}, t_{2}\right)=R\left(h-\left(t_{1}-t_{2}\right) U, \lambda_{r}\right) .
$$


Under these assumptions, equation 6.2 makes explicit that the correlation function evaluated at the time taken for the flow to convect between the upstream root and downstream roots, $t_{1}-t_{2}=h_{r} / U$, i.e., $R=R\left(0, \lambda_{r}\right)$, is only a function of the spanwise separation distance $\lambda_{r}$.

We further make the assumptions that the coherence of the sources has the same frequency to that of the turbulence velocity and that the source strength at the root position $\left(r_{2}, x_{2}\right)$ is identical to that at root position $\left(r_{1}, x_{1}\right)$ and shown in Chaitanya et al. (2017) to be only a function of non-dimensional frequency $\omega h / U$. Further noting from the above argument that the space-time correlation function, and hence the coherence function, is only a function of the spanwise separation distance between roots for frozen, homogeneous turbulent flow, i.e., $\gamma^{2}=\gamma^{2}\left(\lambda_{r}, \Lambda_{t}, \omega\right)$, the cross spectrum between adjacent root sources may therefore be written as,

$$
\mathbf{E}\left[q^{*}\left(r_{1}, x_{1}, \omega h / U\right) q\left(r_{1}+\lambda_{r}, x_{1}+h_{r}, \omega h / U\right)\right]=\overline{q^{2}}(\omega h / U) \gamma^{2}\left(\lambda_{r}, \omega\right),
$$

where $\overline{q^{2}}(\omega h / U)$ is the mean square source strength at the root location.

The radiated sound power $W$ is related to the (integrated) mean square far-field pressure, i.e.,

$$
W(\omega) \propto \overline{p^{2}}(\omega) \propto \mathbf{E}\left[p^{*}(\omega) p(\omega)\right]
$$

Substituting equation 6.1 and 5.7 into equation 5.8, the radiated sound power becomes,

$$
W(\omega) \propto 2 \overline{q^{2}}(\omega h / U)\left[1+\gamma^{2}\left(\lambda_{r}, \Lambda_{t}, \omega\right) \cos \left(\omega h_{r} / U\right)\right] .
$$

Equation 5.9 for the sound power radiated by the double wavelength serration will now be compared to the sound power $W_{s}(\omega)$ radiated by the optimum single-wavelength serration with the same amplitude. This can be calculated within the present theoretical framework by assuming zero phase difference and zero coherence between adjacent roots, i.e., substituting $h_{r}=0$ and $\gamma^{2}\left(\lambda_{r}, \Lambda_{t}, \omega\right)=0$ in equation 5.9 , to give,

$$
W_{s}(\omega) \propto 2 \overline{q^{2}}(\omega h / U)
$$

The additional sound power reduction relative to the optimum single-wavelength serration may be written as $\Delta \mathrm{PWL}_{I}(\omega)=10 \log _{10}(\epsilon)$, where $\epsilon=W_{s}(\omega) / W(\omega)$ (defined to be positive),

$$
\Delta \mathrm{PWL}_{I}(\omega)=-10 \log _{10}\left[1+\gamma^{2}\left(\lambda_{r}, \Lambda_{t}, \omega\right) \cos \left(\omega h_{r} / U\right)\right]
$$

For simplicity, a simple exponential form for the source strength spanwise coherence function was assumed and defined by equation 5.7 of the form,

$$
\gamma^{2}\left(\lambda_{r}, \Lambda_{t}, \omega\right)=e^{-\lambda_{r} / l(\omega)}
$$


where $l(\omega)$ is the frequency-dependent coherence length defined by $l(\omega)=\int_{0}^{\infty} \gamma^{2}\left(\lambda_{r}, \Lambda_{t}, \omega\right) d \lambda_{r}$. Assuming the von Karman turbulence spectra for the incoming turbulent flow, the coherence length is given by (Amiet, 1976)

$$
\frac{l(\omega)}{\Lambda_{t}}=\frac{9 \pi}{\sqrt{1+\left(2 \omega \Lambda_{t} / U\right)^{2}}} \frac{\left(2 \omega \Lambda_{t} / U\right)^{2}}{1+3\left(2 \omega \Lambda_{t} / U\right)^{2}}
$$

Note that, in order to provide best fit to the experimental noise reduction spectra, the coherence length of equation 5.13 includes an additional factor of 3 compared to Amiet's original expression. The justification for this choice of arbitrary constant is that the coherence of the pressure response at the aerofoil leading edge is likely to be greater than that of the turbulence velocity causing it since the aerofoil will not respond equally to all gust components.

The behaviour of equation 5.13 is central to understanding the noise reduction characteristics of double-wavelength serrations. The expression for $l$ above tends to zero as $\left(2 \omega \Lambda_{t} / U\right)^{2}$ as $\omega \Lambda_{t} / U \rightarrow 0$ and as $\left(2 \omega \Lambda_{t} / U\right)^{-1}$ as $\omega \Lambda_{t} / U \rightarrow \infty$. Crucially, it takes a maximum value at $2 \omega \Lambda_{t} / U=1$, from which the frequency $\omega_{\Lambda}$ of maximum coherence is given by,

$$
\omega_{\Lambda}=U / 2 \Lambda_{t}
$$

Equation 5.14 therefore identifies the frequency $\omega_{\Lambda}$ at which adjacent root sources have maximum coherence, which in equation 5.13 gives the maximum value of the coherence length $l\left(\omega_{\Lambda}\right)$ as,

$$
\frac{l\left(\omega_{\Lambda}\right)}{\Lambda_{t}}=\frac{9 \pi}{4 \sqrt{2}} \approx 4.8
$$

Substituting equation 5.14 and 5.13 in 5.11 and re-writing the form of the coherence in terms of $\omega_{\Lambda}$ gives

$$
\Delta \operatorname{PWL}_{I}(\omega)=-10 \log _{10}\left[1+\gamma^{2}\left(\lambda_{r} / \Lambda_{t}, \omega / \omega_{\Lambda}\right) \cos \left(\pi \omega / \omega_{0}\right)\right]
$$

where

$$
\gamma^{2}\left(\lambda_{r} / \Lambda_{t}, \omega / \omega_{\Lambda}\right)=\exp \left(-\frac{\lambda_{r}}{\Lambda_{t}} \frac{\sqrt{1+\left(\omega / \omega_{\Lambda_{t}}\right)^{2}}\left(1+3\left(\omega / \omega_{\Lambda}\right)^{2}\right)}{9 \pi\left(\omega / \omega_{\Lambda}\right)^{2}}\right)
$$

Additional noise reductions afforded by the double-wavelength serration given by equation 5.16 are therefore determined by the ratio of spanwise root separation distance relative to the turbulence length scale $\lambda_{r} / \Lambda_{t}$ and the two non-dimensional frequencies of $\omega / \omega_{0}$ and $\omega / \omega_{\Lambda}$. We now use this simple model to understand the experimental noise reduction spectra plotted above.

Figure 5.8 shows a comparison between the measured additional noise reduction spectra for the case of $\lambda_{r} / \Lambda_{t}=2.34$ and $a / c_{0}=0.13$ with that predicted from Eqs 5.16 and 5.17, plotted against non-dimensional frequency $\omega / \omega_{0}$. The measured spectra was obtained by subtracting $10 \log _{10}(f h / U)+10$ from the measured noise reduction spectra plotted in figure 5.8. A reasonably good comparison is observed between the measured and predicted noise reduction spectra, providing confirmation of the validity of the additional noise reduction 


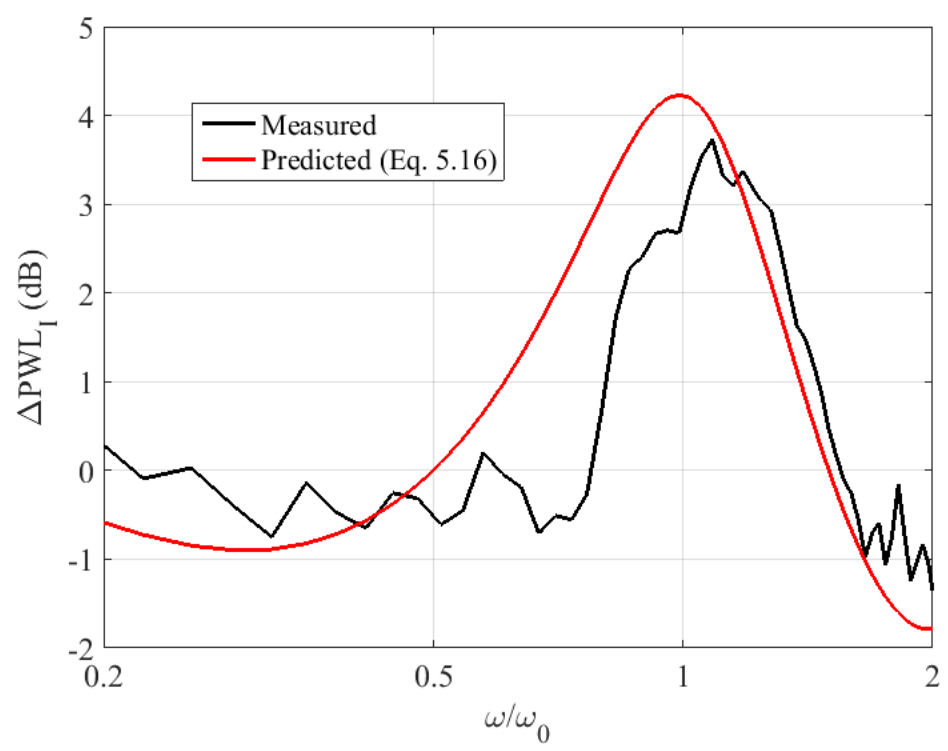

FIGURE 5.8: Additional noise reductions $\left(\Delta \mathrm{PWL}_{I}\right)$ comparison between measured and predicted for flat plates.

mechanism described by the model. Discrepancies between the measured and predicted noise reduction spectra arises mainly from the size and precise location of the root sources, which as pointed by Turner et al. (2016), are not exactly located at the root. Nevertheless, this level of agreement proves unequivocally that the additional benefit of double wavelength serrations is a result of interference between two compact sources located at adjacent roots.

\subsection{Self-similarity of double-wavelength serrations}

The expressions above for the two components of noise reduction $\Delta \mathrm{PWL}_{S}$ and $\Delta \mathrm{PWL}_{I}$ can all be expressed in terms of the non-dimensional frequency $f L / U$ where $L$ takes the three characteristic lengths of $h, h_{r}$ and $\Lambda_{t}$. Overall noise reductions are therefore determined by the ratio of hydrodynamic wavelength $U / f$ to these three dimensions. Double-wavelength serrations for which these four dimensions are in constant ratio are therefore predicted to produce identical noise reductions. The noise reduction mechanism may therefore be regarded as self-similar in these four length-scales.

An important consequence of this observation is that noise reductions obtained for a fixed geometry and integral length scale should be identical at all flow speeds when plotted against non-dimensional frequency $f L / U$, where $L$ can be any of the length scales $h, h_{r}$ and $\Lambda_{t}$.

To confirm this prediction, the noise reduction spectra are shown in figure 5.9 plotted against $\omega / \omega_{0}$, i.e. for $L=h_{r}$, at the four different velocities of $U$ equal to 20,40,60 and $80 \mathrm{~m} / \mathrm{s}$ for the typical double-serration wavelength example of $\lambda_{r} / \Lambda_{t}=2.34$ and $h_{r} / c_{0}=0.13$.

As predicted the noise reduction spectra are almost identical to within $1 \mathrm{~dB}$ with greatest deviation being observed at the highest frequencies where self noise starts to dominate. 


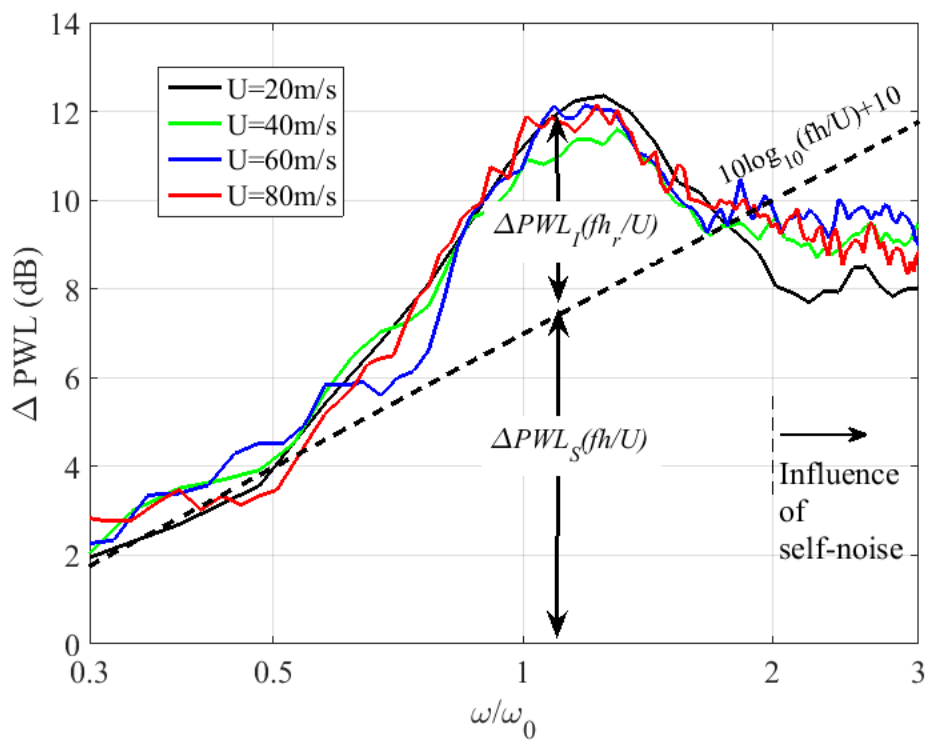

FIGURE 5.9: Influence of flow velocity on noise reduction due to doublewavelength serration profiles.

\subsection{Predicted dependence of $\Delta \mathbf{P W L}_{I}$ on $h_{r}$ and $\lambda_{r}$ on noise reduc- tions}

In this section we interrogate the model developed above to understand more fully the variation in the additional noise reductions observed in the measured noise spectra plotted in figure 5.8 to variations in the double wavelength parameters of $h_{r}$ and $\lambda_{r}$.

Setting equation 5.16 to be greater than $10 \log _{10}(\epsilon)$, gives the following condition necessary to achieve an additional sound power level reduction of $10 \log _{10}(\epsilon) \mathrm{dB}$ greater than the maximum noise reduction that can be achieved using a single-wavelength serration,

$$
\gamma^{2}\left(\lambda_{r} / \Lambda_{t}, \omega / \omega_{\Lambda}\right) \cos \left(\pi \omega / \omega_{0}\right)<\frac{1-\epsilon}{\epsilon}
$$

To identify the combinations of $\lambda_{r}$ and $h_{r}$ that provides the noise reduction of $10 \log _{10}(\epsilon)$, $\Delta \mathrm{PWL}_{I}$ from equation 5.16 is plotted in figure 5.10 against $\omega / \omega_{0}$ for the same six different spanwise distances $\lambda_{r} / \Lambda_{t}$ plotted previously in figure 5.6 of between 1.17 to 7 . Just as in the measured noise reduction spectra in figure 5.6, the peak-to-root amplitude is held constant at $2 h / c_{0}=0.33$. Also plotted in this figure by way of a reference value, as a dashed line, is the noise reduction of $3 \mathrm{~dB}$.

This figure confirms the general dependence in the noise reduction spectra with $\lambda_{r}$ observed experimentally in figure 5.6. Maximum noise reductions at the peak frequency $\omega_{0}$ are predicted to diminish sharply as $\lambda_{r}$ increases, confirming the need for high levels of coherence between adjacent root sources for effective interference to occur. Another significant feature in figure 5.10 is that noise reductions are fundamentally limited to a number of finite frequency bandwidths, which are determined from a combination of the effect of interference between adjacent roots, as determined by the factor $\cos \left(\pi \omega / \omega_{0}\right)$, and the coherence between adjacent roots sources, as determined from the factor $\gamma^{2}\left(\lambda_{r} / \Lambda_{t}, \omega / \omega_{\Lambda}\right)$. The relationship between the noise reduction frequency-bandwidth $\omega_{2}-\omega_{1}$ and the geometrical parameters of the double wavelength serration are discussed in detail in Section 5.8.3. 


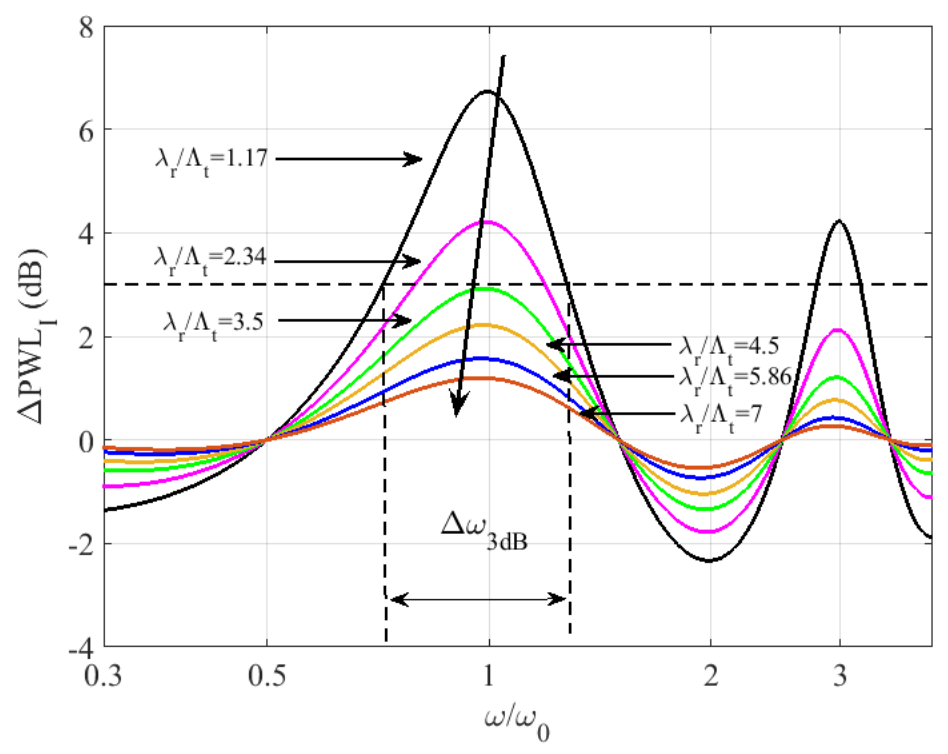

FIGURE 5.10: Predicted behaviour of $\Delta \mathrm{PWL}_{I}$ on $h_{r}$ and $\lambda_{r}$.

In both the measured noise reduction spectra in figures 5.6 and their predictions in figure 5.10 it is fundamentally important to recognize that the main reason that the serration geometries under investigation are effective in reducing noise is that the peak frequency $\omega_{0}$ was chosen to equal the frequency $\omega_{\Lambda}$ at which the coherence length is a maximum. The geometries investigated experimentally in figure 5.6 therefore represent the optimum condition, which we shall discuss in detail in the section 5.8 .2 below.

We first discuss the factors that affect the noise reduction at the tuned frequency $\omega_{0}$ at which adjacent roots sources are $180^{\circ}$ out of the phase. We then proceed to discuss the factors that determine the frequency bandwidth $\omega_{2}-\omega_{1}$ of noise reductions.

\subsubsection{Noise reductions at the tuned frequency $\omega_{0}$}

Additional noise reductions $\triangle \mathrm{PWL}_{I}$ due to double-wavelength serrations are determined by just two geometric parameters; the spanwise and streamwise separation distances between adjacent roots, $\lambda_{r}$ and $h_{r}$ respectively. We now summarise the relationship between these parameters and the noise reduction performance at the tuned frequency $\omega_{0}$, which represents the maximum possible noise reduction.

Equation 5.16 enables the additional sound power reduction to be compared directly to the noise reduction due to the optimum single-wavelength serration at the peak frequency $\omega_{0}$,

$$
\Delta \mathrm{PWL}_{I}(\omega) \leq \Delta \mathrm{PWL}_{I}\left(\omega_{0}\right)=-10 \log _{10}\left(1-\gamma^{2}\left(\lambda_{r} / \Lambda_{t}, \omega_{0} / \omega_{\Lambda}\right)\right)
$$

which of course is determined solely by the degree of coherence between adjacent root sources.

At frequency $\omega_{0}$, the essential requirement for good levels of additional noise reduction is that adjacent root sources must be sufficiently coherent. At this frequency $\cos \left(\pi \omega / \omega_{0}\right)=-1$ and therefore equation 5.18 imposes a lower limit on the coherence function of $\gamma^{2}\left(\lambda_{r}, \Lambda_{t}, \omega_{0}\right)=$ $(\epsilon-1) / \epsilon$ necessary to achieve an additional noise reduction of at least $10 \log _{10}(\epsilon)$. For 
example, to provide a noise reduction of $3 \mathrm{~dB}(\epsilon=2)$ this condition may be written as $e^{-\lambda_{r} / l\left(\omega_{0}\right)}>0.5$ and hence $\lambda_{r}$ must not exceed $-(\log 0.5) l\left(\omega_{0}\right)$. Noting the expression for $l(\omega)$ in equation 5.13, the maximum value of $\lambda_{r}$ necessary to ensure a noise reduction at the tuned frequency of at least $3 \mathrm{~dB}$ must therefore satisfy

$$
\lambda_{r} \leq 0.7 l\left(\omega_{0}\right) \quad\left(\Delta \mathrm{PWL}\left(\omega_{0}\right)>3 \mathrm{~dB}\right)
$$

\subsubsection{Optimum condition $\left(\omega_{0}=\omega_{\Lambda}\right)$}

As stated explicitly in equation 5.19, maximum noise reductions are obtained at the tuned frequency $\omega_{0}$. However, if $\omega_{0}$ is chosen (by the choice of $h_{r}$ ) to deviate too far from the frequency $\omega_{\Lambda}$ of maximum coherence length, the condition $\lambda_{r} \leq 0.7 l\left(\omega_{0}\right)$ can only be satisfied by excessively small $\lambda_{r}$ since $l$ tends to zero at frequencies away from $\omega_{\Lambda}$. Optimum noise reductions are therefore obtained at the tuned frequency, at which adjacent root sources are, $180^{\circ}$ out of phase, when it is chosen to coincide with the frequency of maximum coherence, i.e.,

$$
\omega_{0}=\omega_{\Lambda}
$$

Equating equations 6.11 and 5.14 therefore provides the optimum value of $h_{0}$ in terms of the turbulence length-scale at which $\omega_{0}=\omega_{\Lambda}$,

$$
h_{0}=2 \pi \Lambda_{t}\left(\omega_{0}=\omega_{\Lambda}\right)
$$

The effect on noise reductions at a tuned frequency $\omega_{0}$ less than, or greater than, $\omega_{\Lambda}$ is illustrated in figure 5.11, which shows plots of $-\cos \left(\pi \omega / \omega_{0}\right)$ and $\gamma^{2}$ versus $\omega / \omega_{0}$ for the three cases of $\omega_{0} / \omega_{\Lambda}<1,=1$ and $>1$. At sub-optimum conditions, $\omega_{0} / \omega_{\Lambda} \neq 1$, the frequencies of the peaks due to the two terms differ, while at the optimum condition $\omega_{0} / \omega_{\Lambda}=1$, the two peak frequencies coincide resulting in maximum noise reduction. Note that it is also possible to obtain the optimum condition at the second harmonic frequency, i.e., $3 \omega_{0}=\omega_{\Lambda}$. This is the situation in Figure 5.5 for which greatest noise reductions are observed at the second harmonic frequency for the case of $\phi=\pi / 2$.

Finally, combining equation 5.15 and 5.20 gives a simple expression for the upper limit of $\lambda_{r}$ at which additional noise reductions of at least $3 \mathrm{~dB}$ may be obtained in terms of the turbulence integral length scale,

$$
\lambda_{r} \leq 0.7 l\left(\omega_{\Lambda}\right) \approx 3.3 \Lambda_{t}
$$

This prediction is close to the observed value in figure 5.6 of roughly $2.5 \mathrm{~dB}$ for the slightly wider serration of $\lambda_{r} / \Lambda_{t}=3.5$.

\subsubsection{Noise reduction frequency bandwidth}

Double-wavelength serrations are able to provide noise reductions over and above the maximum noise reduction achievable with a single-wavelength serration. However, as both measurements and predictions have shown, these additional noise reductions are fundamentally limited to finite frequency bandwidths. This finding is a consequence of the fact 


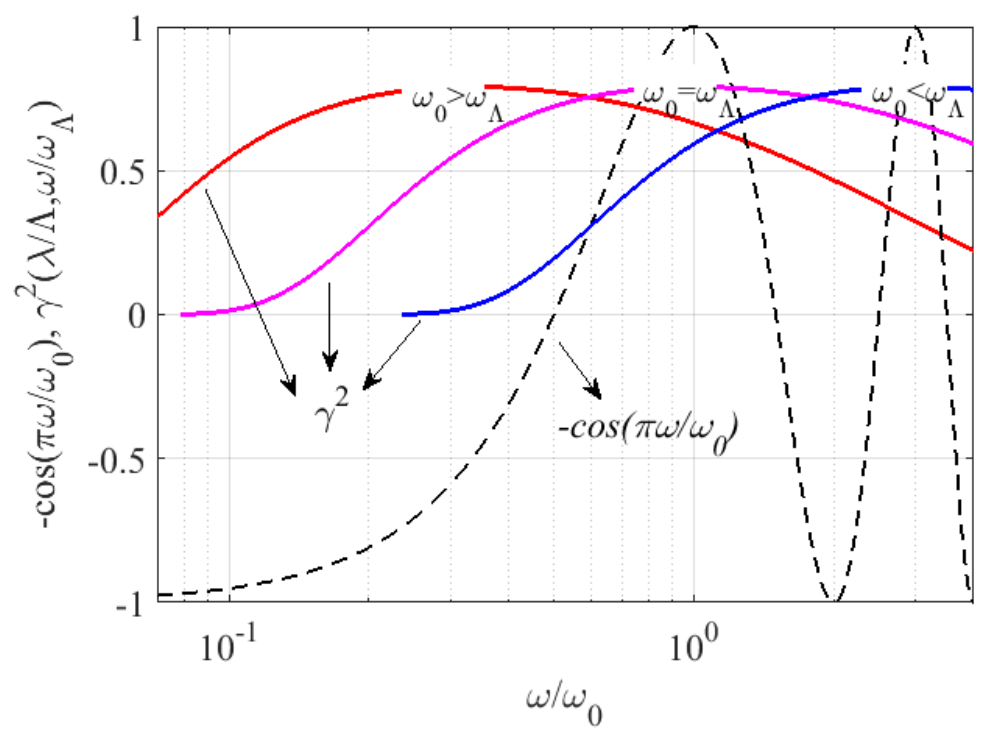

FIGURE 5.11: Condition for optimum noise reductions.

that the frequency range at which adjacent root sources are sufficiently out of phase and also have sufficiently high coherence are themselves band-limited.

The measured noise reductions in Figure 5.6 and the predicted reductions from figure 5.10 both indicate that the frequency bandwidth increases as $\lambda_{r}$ is reduced. More formally, the upper and lower frequency limits, $\omega_{1}$ and $\omega_{2}$, either side of $\omega_{0}$, at which an additional noise reduction of $10 \log _{10}(\epsilon)$ is obtained may be deduced from the two solutions to equation 5.18 either side of $\omega_{0}$, which we express here in terms of the tuned frequency $\omega_{0}$ as

$$
\frac{\omega_{1,2}}{\omega_{0}}=\frac{1}{\pi} \cos ^{-1}\left(\frac{(1-\epsilon)}{\epsilon} \frac{1}{\gamma^{2}\left(\lambda_{r} / \Lambda_{t}, \omega_{1,2} / \omega_{\Lambda}\right)}\right)
$$

The frequency bandwidth $\Delta \omega_{3 d B}=\omega_{1}-\omega_{2}$ of $3 \mathrm{~dB}$ additional noise reductions was calculated from solutions to Equation 5.24 for the three examples, $\omega_{0} / \omega_{\Lambda}<1,=1$ and $>1$ and plotted against $\lambda_{r} / \Lambda_{t}$ in figure 5.12. As expected, the highest frequency bandwidth is achieved at the optimum condition $\omega_{0}=\omega_{\Lambda}$. The fundamental upper limit on the frequency bandwidth of $3 \mathrm{~dB}$ additional noise reductions is achieved as $\lambda_{r}$ tends to zero and the coherence between adjacent root sources approaches unity. In this limit, the upper and lower frequencies are $\omega_{1} / \omega_{0}=2 / 3, \omega_{2} / \omega_{0}=4 / 3$ and therefore the upper limit on the frequency bandwidth $\Delta \omega_{3 d B}=\left(\omega_{2}-\omega_{1}\right)$ at additional noise reduction $\Delta \mathrm{PWL}_{I}=3 \mathrm{~dB}$ is given by

$$
\frac{\Delta \omega_{3 d B}}{\omega_{0}}=\frac{2}{3}
$$

The upper limiting frequency bandwidth is therefore proportional to the tuned frequency $\omega_{0}$. Tuning to a low frequency, therefore only produces noise reductions over a small frequency bandwidth in absolute terms. 


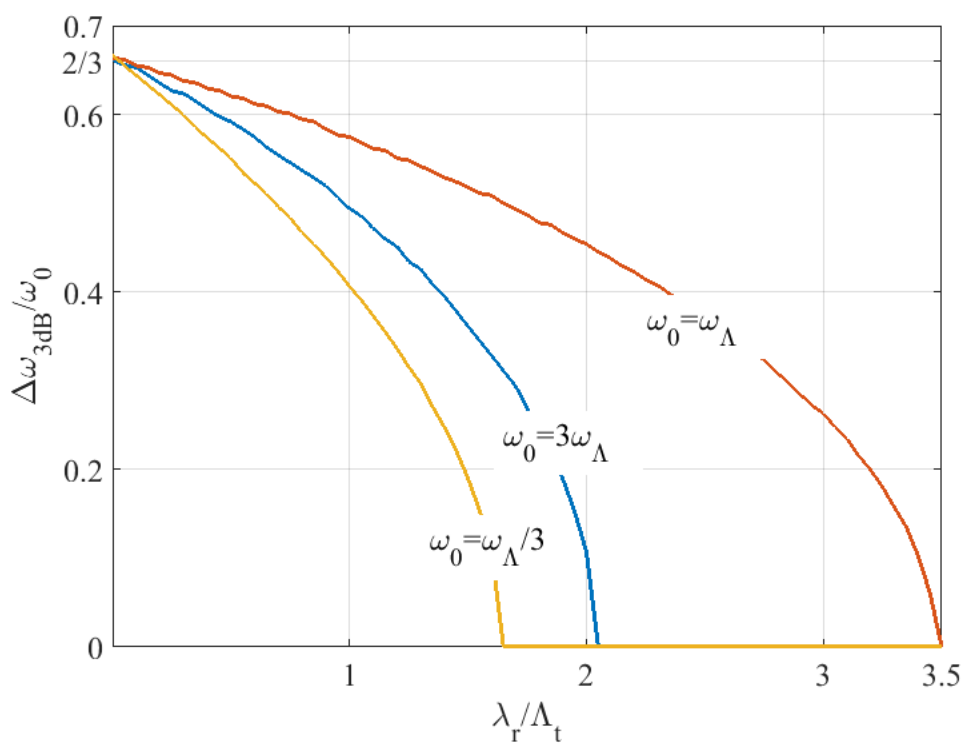

FIGURE 5.12: Additional noise reductions frequency bandwidth

\subsection{Sound Power Spectra comparison for 3-D aerofoils}

Finally, we validate the noise reduction principle established on flat plates in Section 5.4 by applying it to the leading edge of a NACA65 aerofoil with 10\% thickness, $150 \mathrm{~mm}$ mean chord and $450 \mathrm{~mm}$ span. Noise measurements were made on the aerofoil with a doublewavelength serration comprising of two single-wavelengths of $\left(\lambda_{1} / c_{0}=0.067\right.$ and $\lambda_{2} / c_{0}=$ 0.13 ) with zero phase difference. Summing these two single-wavelength components gives a total peak to root distance of $2 h / c_{0}=0.33$, a root-to-root spanwise and streamwise distance $\lambda_{r} / c_{0}=0.059$ and $h_{r} / c_{0}=0.13$ respectively. In terms of the important non-dimensional ratios identified above, these distances correspond to $\lambda_{r} / \Lambda_{t}=2.34, \omega_{0} / \omega_{\Lambda} \approx 1$, where the tuned frequency $\omega_{0}$ corresponds to $1530 \mathrm{~Hz}$ at the flow speed of the measurement $U=60 \mathrm{~m} / \mathrm{s}$. Also measured for comparison were the noise reductions for the two single-wavelength serrations of the same peak-to-root amplitude $2 h$ together with the baseline (un-serrated) aerofoil. All aerofoils were fabricated using a 3-D printer, which provides smooth surface finish.

The serrated aerofoils are defined such that if $y(X)=f(X)$ defines the NACA65 aerofoil profile, where $X=0$ represents the trailing edge and $X=1$ the leading edge. The profile $y(x, r)$ at any spanwise position $r$ along the aerofoil is given by,

$$
y(x, r)= \begin{cases}f\left(x / c_{0}\right), & 0<x / c_{0}<2 / 3 \\ f(x / c(r)), & 2 / 3<x / c(r)<1\end{cases}
$$

where the chord is a function of span $r$, i.e., $c(r)=c_{0}+h_{\text {eff }} \sin \left(2 \pi r / \lambda_{1}\right)+h_{\text {eff }} \sin \left(2 \pi r / \lambda_{2}\right)$ and $\mathrm{x}$ varies between 0 at the trailing edge and the leading edge $x=c(r)$. The total peak-toroot distance of $2 h$ is maintained by adjusting $h_{e f f}$.

Figure 5.13 shows the comparison of the sound power reduction spectra between the two single-wavelength serrations and the double-wavelength serration when introduced onto 


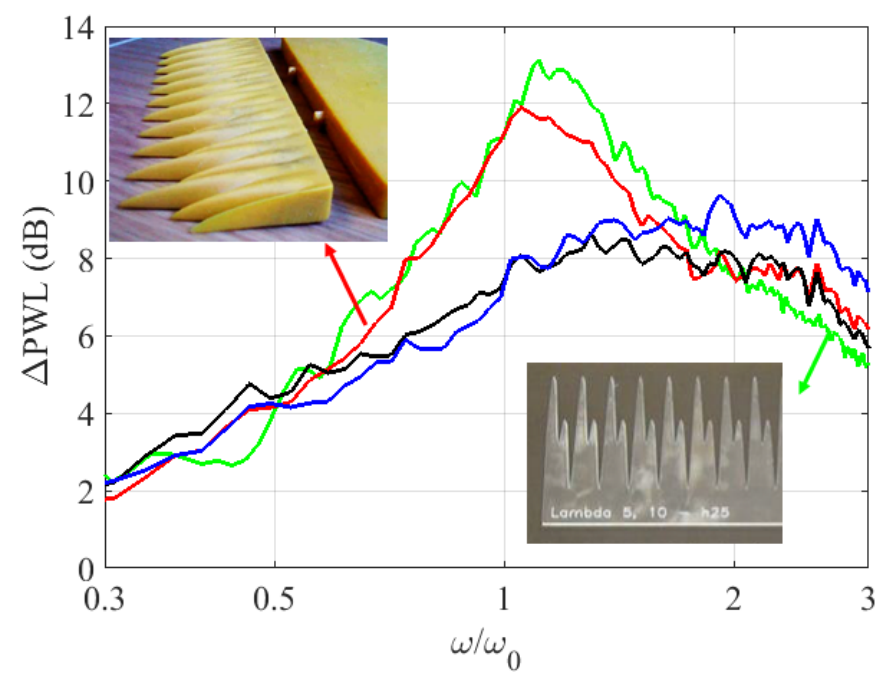

FIGURE 5.13: Total sound power reductions level ( $\Delta$ PWL) for single, doublewavelength serrations on 3-D aerofoils of $\lambda_{1} / c_{0}=0.067 \& \lambda_{2} / c_{0}=0.13$, $\lambda_{r} / \Lambda_{t}=2.65,2 h / c_{0}=0.33, \mathrm{U}=60 \mathrm{~m} / \mathrm{s}$. (black line: Single-wavelength, $\lambda_{1}$ (Aerofoil); blue line: Single-wavelength, $\lambda_{2}$ (Aerofoil); red line: Doublewavelength (Aerofoil); green line: Double-wavelength (Flat plate))

the aerofoil and also on a flat plate. Differences between the respective spectra for the aerofoil and a flat plat are typically about $1 \mathrm{~dB}$ confirming that the flat plate encapsulates the same physical principles as the 3-D aerofoil. Furthermore, it confirms the validity of the new noise reduction principle on relatively thick aerofoil geometries.

\subsection{Aerodynamic performance}

Previous sections have highlighted the potential effectiveness of leading edge serrations on 3D aerofoils. However, it is imperative that aerodynamic performance is not substantially degraded by their introduction. In this section the effect of leading edge serrations on the steady aerodynamic performance of aerofoils is investigated experimentally. The lift and drag forces were measured on the NACA65 aerofoil with varying serration wavelength $\left(\lambda_{r} / c_{0}\right)$. The geometric angle of attack varies from $-2.5^{\circ}$ to $10^{\circ}$ but due to jet deflection in the open jet wind tunnel the corresponding effective angle of attack is in the smaller range $-1^{\circ}$ to $2.8^{\circ}$. Aerodynamics measurements were performed on same open jet wind-tunnel as acoustic measurements to ensure consistency.

Figure 5.14a shows the coefficient of lift for the baseline and serrated aerofoil of amplitudes $\left(h / c_{0}\right) 0.167$ for two different serration wavelengths $\lambda_{1} / c_{0}$ and $\lambda_{2} / c_{0}$ of $0.067,0.13$ compared against double-wavelength serrations. Whilst the gradient of lift coefficient versus angle of attack remains unchanged by the introduction of serrations (Gradient $\approx 6.1$ /radian), levels are consistently smaller by between 0.01 and 0.05 compared to the baseline case. Thus, whilst increasing the serration wavelength, the corresponding lift performances improves and tend towards the performance of the baseline aerofoil. The lift performance of the double-wavelength serrations falls in between that of the two single frequency serrations. The corresponding variation in drag coefficient is shown in Figure 5.14b which is observed to increase by between 0.001 and 0.005 compared to the baseline case. The variation is very 


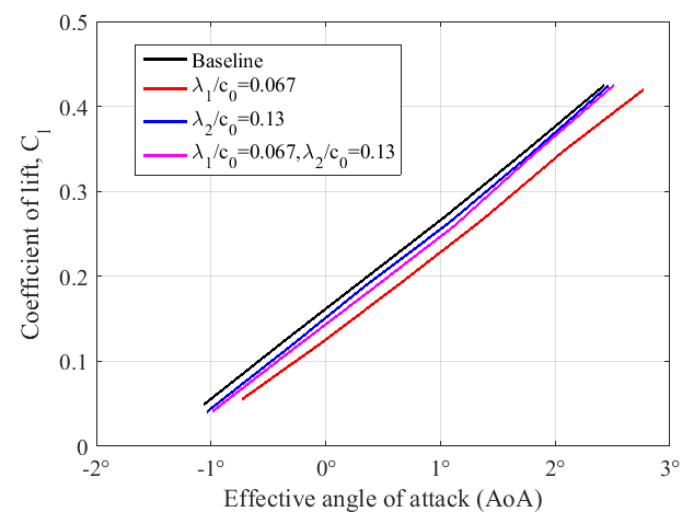

(a) Coefficient of lift.

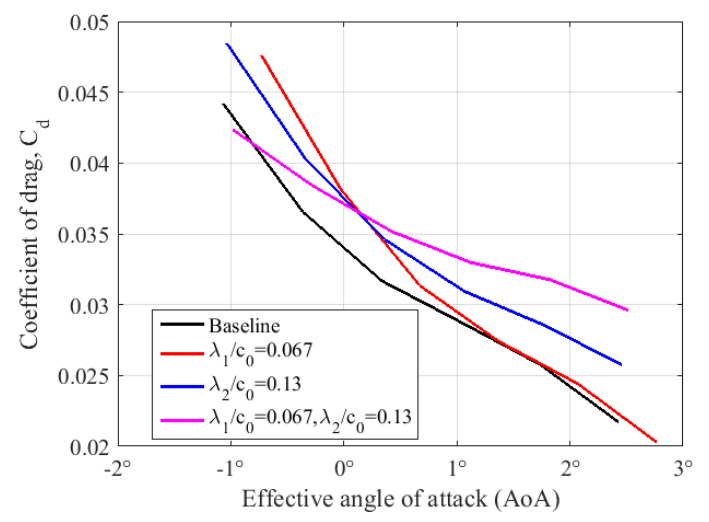

(b) Coefficient of drag.

FIGURE 5.14: Aerodynamic evaluation of serrated aerofoils $\left(\lambda_{r} / c_{0}=0.067\right)$ at the jet velocity $U=60 \mathrm{~m} / \mathrm{s}$.

small, hence no conclusions can be drawn but, very roughly, the double-wavelength generate slightly greater drag compared to the single-wavelength serrations. As mentioned earlier in section 4.5, the ambiguity in the absolute values of lift and drag coefficients are due to end effects in the open jet wind tunnel facility. However, the purpose of this measurement is to demonstrate the relative difference between single-wavelength and double-wavelength serration.

\subsection{Conclusion}

A new noise control principle has been developed and validated for the reduction of broadband interaction noise on aerofoils. The new control method is capable of providing substantially better noise reduction that the maximum noise reduction achievable on singlewavelength serration geometries. A simple analytic model is proposed to explain the additional noise reduction over and above that due to single-wavelength profiles. This additional noise reduction has been shown to depend on just the three non-dimensional factors of $\omega / \omega_{0}, \omega / \omega_{\Lambda}, \lambda_{r} / \Lambda_{t}$. In summary, therefore, the total noise reduction from the double wavelength serration can be written as the sum of a reduction due to the source strength at the root and additional noise reductions due to interference between adjacent root sources,

$$
\Delta \operatorname{PWL}(\omega)=10 \log _{10}(f h / U)-10 \log _{10}\left(1+e^{-\lambda_{r} / l(\omega)} \cos \left(\pi \omega / \omega_{0}\right)\right)+10
$$

The optimum condition at which maximum noise reductions are obtained at the tuned frequency $\omega_{0}$ has been identified. It is shown to occur at the frequency $\omega_{0}$ at which adjacent root sources are simultaneously $180^{\circ}$ out of phase and have maximum coherence length at $\omega_{\Lambda}$. An upper frequency bandwidth at which additional noise reductions of at least $3 \mathrm{~dB}$ are obtained has been identified as $2 \omega_{0} / 3$. Finally, the double wavelength geometry has been applied to a relatively thick aerofoil and shown to provide almost identical noise reductions to that when the same geometry is applied to a flat plate.

In conclusion a new noise control principle for the reduction of aerofoil leading edge noise has been proposed. It has been validated experimentally and shown to provide considerably better noise reductions that single-wavelength profile alone. Further work is needed to 
establish the aerodynamic performance of such leading edge geometries although there is no reason to believe that these profiles will perform any worse than single-wavelength serrations. These profiles also pose a challenge to structural engineers to ensure that there is no reduction in structural integrity due to stress concentrations at the roots. This work opens up the possibility of research into the development of a new family of leading edge profiles based on the same noise reduction principle of destructive interference between dominant compact sources distributed over the leading edge. 



\title{
Chapter 6
}

\section{Slitted leading edge profiles for the reduction of turbulence-aerofoil interaction noise}

\author{
Contents

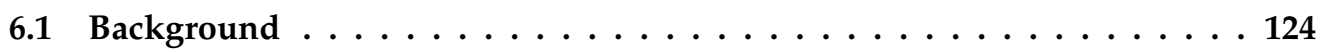 \\ 6.2 Acoustic performance of sawtooth serrations . . . . . . . . . 124 \\ 6.3 Slitted-root profiles . . . . . . . . . . . . . . . . . . . . 125 \\ 6.3 .1 Geometry . . . . . . . . . . . . . . . . . . 125 \\ 6.3.2 A simple analytical model to predict noise reductions . . . . . . . . 127 \\ 6.3 .3 Limiting case . . . . . . . . . . . . . . . . . . . . . . . . . 129 \\ 6.3.4 Acoustic performance on flat plate . . . . . . . . . . . . . . 130 \\ 6.3.5 Influence of $w$ on additional noise reductions . . . . . . . . . . 131 \\ 6.3.6 Influence of $h_{s}$ on additional noise reductions . . . . . . 133 \\ 6.3.7 Comparison between predicted and measured additional noise re- \\ ductions . . . . . . . . . . . . . . . . . . . . . . 135 \\ 6.3.8 Sound Power Spectra comparison for 3D aerofoils . . . . . . . . . 137

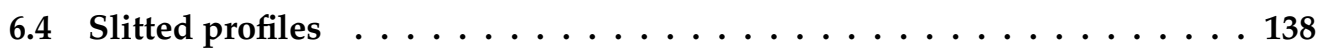 \\ 6.4 .1 Geometry . . . . . . . . . . . . . . . . . . . 139 \\ 6.4.2 Influence of $a$ and $w$ on noise reductions . . . . . . . . . . 140 \\ 6.4 .3 Optimum sound power redictions . . . . . . . . . . . . . . . . 141 \\ 6.4.4 Sound Power Spectra comparison for 3D aerofoils . . . . . . . . . . . 141 \\ 6.5 V-slit serrations . . . . . . . . . . . . . . . . . . . . . . . . 142 \\ 6.5 .1 Geometry . . . . . . . . . . . . . . . . . . 143 \\ 6.5.2 Acoustic performance on flat plate aerofoils . . . . . . . . . 143 \\ 6.6 Conclusions . . . . . . . . . . . . . . . . . . . 144
}

In the previous chapter a new noise control principle was presented and validated against experimental data for the reduction of broadband interaction noise on aerofoils. It relied on a new control concept on aerofoils related to the interference between adjacent root sources. The new profile was found to provide considerably better noise reductions than singlewavelength profiles alone. However, the limiting factor in determining the maximum noise reductions was found to be due to the imperfect coherence between adjacent root sources.

In this chapter, new leading edge geometries are developed which will be shown to provide greater noise reductions than even the double-wavelength profiles, with one being designed to incur a smaller aerodynamic penalty. This leading edge geometry is based on the same 
noise reduction principle of destructive interference (demonstrated in chapter 5) between dominant compact sources distributed over the leading edge.

\subsection{Background}

In Chapter 5, it was shown that the double-wavelength serration provides considerably better noise reductions compared to single wavelength profiles at the optimum wavelength. However, these profiles fundamentally limited by the coherence between adjacent roots, thereby requiring the separation distance between adjacent roots to be excessively small. This new leading edge profiles presented in this chapter are designed to overcome some of these issues. However, this design will be shown to provide superior noise reductions compared to the double wavelength of the same peak-to-root distance.

In this chapter, we propose a leading edge geometry for reducing leading edge interaction noise that significantly exceeds the noise reduction performance (by up to $11 \mathrm{~dB}$ ) of both single and double-wavelength serrations. In the most general form it comprises a sawtooth profile in which narrow slits with typical widths of just a few millimetres are introduced at the root positions. The introduction of these slits, upon interaction with turbulent flow, produces two compact acoustic source regions at either ends of the slit. However, since these sources are aligned in the streamwise direction they are highly coherent and their level of destructive interference considerably greater than that of the double wavelength serration. As in the case of the double wavelength serrations, these sources will radiate out of the phase by virtue of the time taken for eddies to convect along the length of the narrow slit. A frequency therefore exists at which these two coherent source regions radiate $180^{\circ}$ out of phase. Providing the source strengths at this frequency are arranged to be equal through appropriate choice of slit width, therefore, perfect cancellation of the sound field can in principle be achieved at this frequency. We will show below that noise reductions of almost $18 \mathrm{~dB}$ in radiated sound power can be achieved using this control principle at a flow speed of $40 \mathrm{~m} / \mathrm{s}$, with a small reduction in performance being observed at higher flow speeds.

By contrast to Chapters 4 and 5, which are based on single wavelength profiles, this new profile is based around sawtooth profiles. It will be shown below that the single wavelength and sawtooth profiles provide almost identical noise reduction at $\omega / \omega_{0}<1$.

In the current chapter, similar to previous chapters, the results of a systematic parametric study are presented on flat plates and later applied to a 3-D aerofoil and shown to have almost the same noise reduction effectiveness as when applied to flat plates. As previously described in Chapter 4 and 5, a $2 \mathrm{~mm}$ thick metallic flat plate with dimensions $(150 \times 450$ $\mathrm{mm}$ ) with a slot is used to insert the serration profiles made from acrylic sheet of $2 \mathrm{~mm}$ thickness are used. This chapter provides an preliminary assessment of the new profiles which represent a completely new approach to the design of leading edge geometries for reduction of turbulence-aerofoil interaction noise.

\subsection{Acoustic performance of sawtooth serrations}

The noise reductions due to sawtooth serrations are first compared with sinusoidal serrations of the same amplitude and wavelength. Due to ease of design sawtooth serrations were considered as the baseline for the rest of the designs investigated in this chapter. Figure 6.1 compares the noise reductions versus non-dimensional frequency $f(2 h) / U$ due 


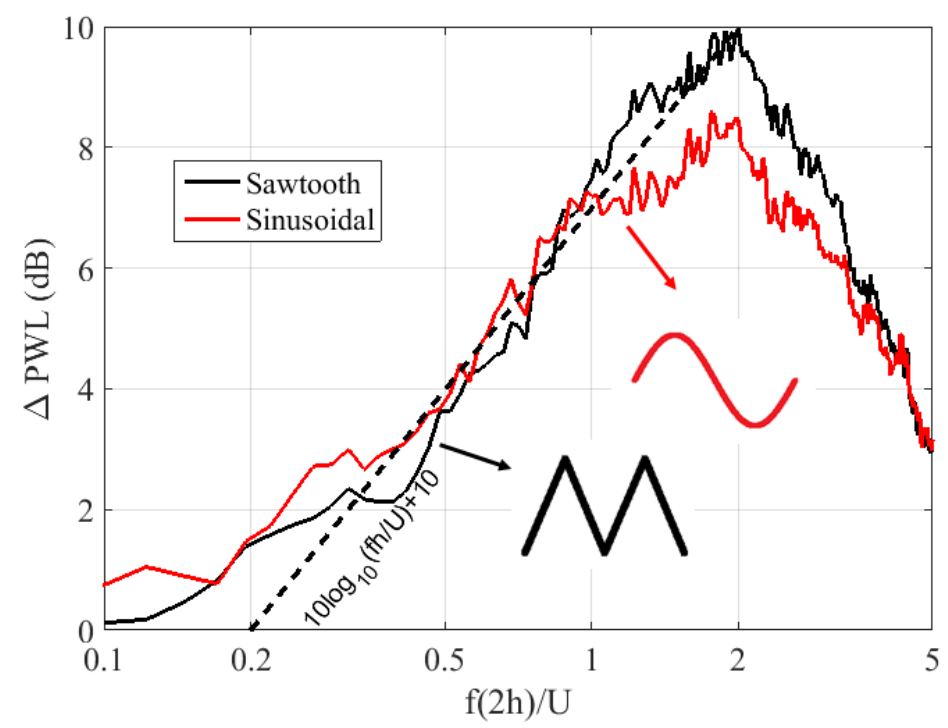

FIGURE 6.1: Comparison of noise reductions between sinusoidal and sawtooth aerofoil.

to a sawtooth and sinusoidal serrations of the same wavelength $\lambda / c_{0}=0.1$ and amplitude $h / c_{0}=0.167$. The wavelengths are chosen to be 4 times the integral length-scale $\Lambda$ of the incoming turbulence, identified by Chaitanya et al. (2017). This wavelength was shown to be the optimal wavelength for providing maximum noise reductions given by $10 \log _{10}(f h / U)+10$ for single-wavelength profiles, shown in this figure as the dashed line.

Nearly identical noise reductions are obtained at frequencies less than $f(2 h) / U=1$. Below this frequency, sawtooth serrations provide consistently poorer noise reductions $(<1 \mathrm{~dB})$ compared to the corresponding sinusoidal case. The poorer noise reductions for the sawtooth serration is most likely because the source strength at the peak is lower than that of the root and hence destructive interference is less effective in reducing noise compared with the single wavelength serration for which peak and root are more similar, in this low frequency range. This behaviour will be exploited in section 7.3 in the chopped-peak serration design. Above this frequency $f(2 h) / U=1$, sawtooth serrations provide consistently better noise reductions compared to the corresponding sinusoidal case. The additional noise reduction is most likely due to lower source strength near the sawtooth root compared to the sinusoidal case owing to the singular behavior of the geometry exactly at the root. However, the source distribution on sawtooth serrations remains to be investigated numerically similar to the investigation presented by Kim et al. (2016) for sinusoidal serrations.

\subsection{Slitted-root profiles}

\subsubsection{Geometry}

The Slitted-root leading edge design investigated in this chapter involves introducing narrow slits at the serration roots, as sketched in figure 6.2. In this chapter we demonstrate that, for very narrow slits, the noise reduction is almost identical at low frequencies, and better at high frequencies, than that of single-wavelength or sawtooth serration profiles of the same peak-to-root amplitude. However, introducing narrow slits clearly requires much less modification to the aerofoil leading edge as shown in Figure 6.3 showing a comparison 
of the two leading edge geometries drawn to scale. However, the main innovation of the new leading edge design is that a new noise reduction mechanism is introduced once the slit width is increased. In this case, the source strengths at either ends of the slit become comparable and interfere destructively, leading to much greater levels of noise reduction compared to conventional profiles of the same overall amplitude.

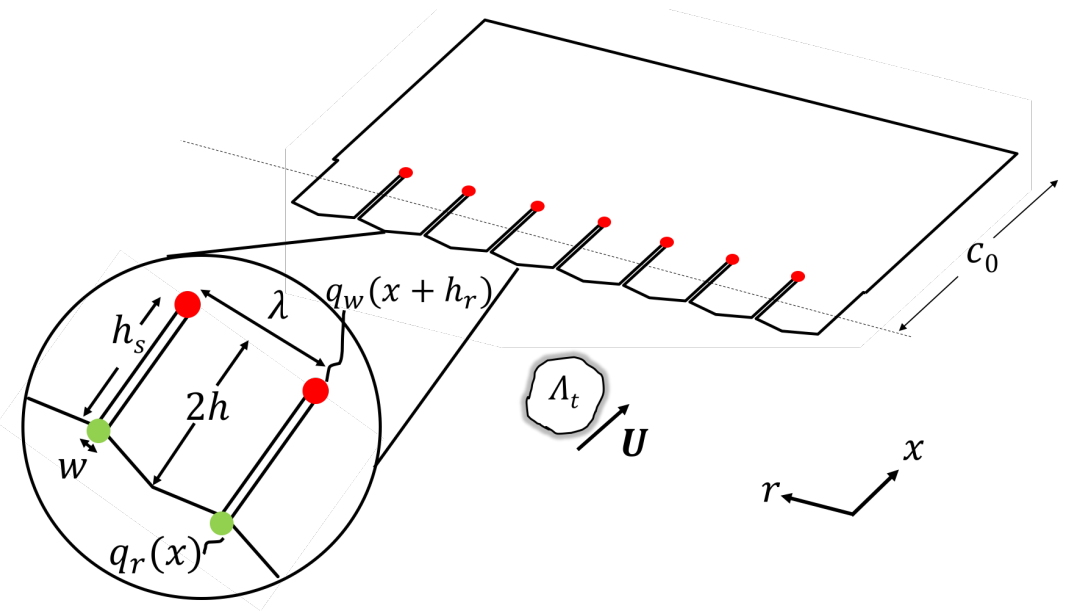

FIGURE 6.2: A sketch of the slitted-root leading edge serration.

The principal dimensions of the slitted root profile are sketched in figure 6.3. For sufficiently large slit widths $w$, the noise reduction spectra shown in Section 6.3.4 exhibit a frequency of maximum noise reduction. This suggests that the location of the dominant sources (indicated as red and green dots) are located at either ends of the slit separated in the streamwise direction by close to the slit height $h_{s}$. The overall distance between the peak and the end of the slit is $2 h$, with the pattern repeating with periodicity (wavelength) $\lambda$.

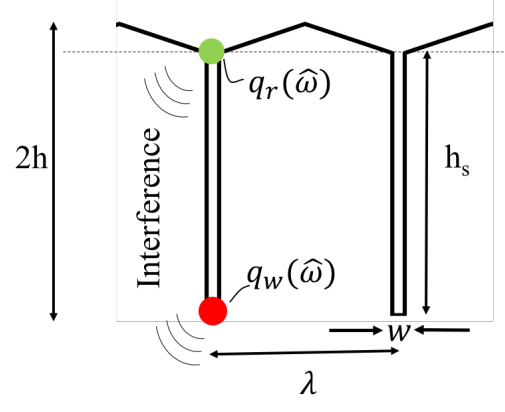

Slitted-root serrations

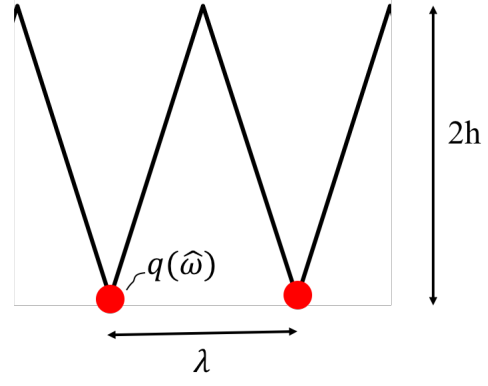

Sawtooth serrations

FIGURE 6.3: A comparison of slitted-root and sawtooth serration.

A photograph of typical slitted-root serration inserts investigated in this chapter are shown in Figure 6.4.

Before presenting the measured noise reduction spectra we first present a simple theoretical framework for describing the mechanism underlying the additional noise reductions compared to conventional sawtooth leading edge serrations. In the next section the compact source model developed in chapter 5 is extended to help explain the additional noise reductions due to slitted-root serration profiles. 


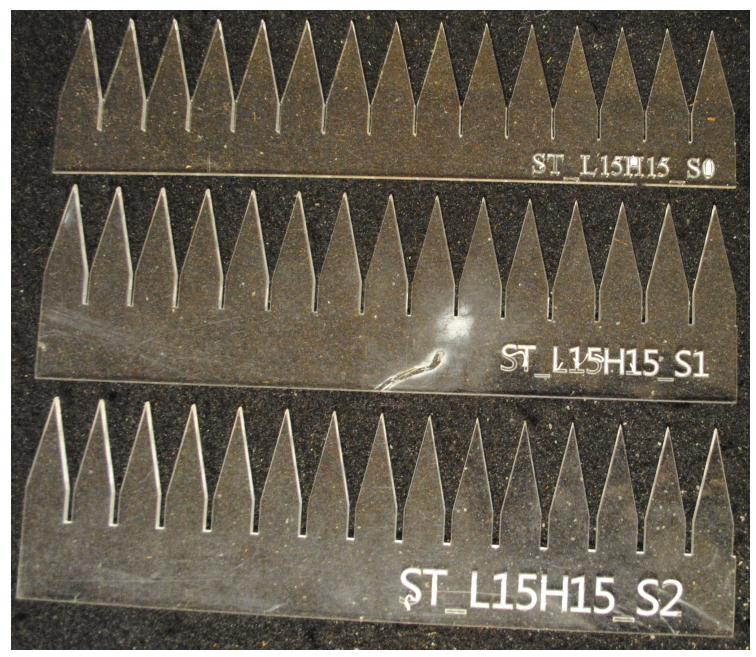

FIGURE 6.4: A typical flat plate slitted-root serration inserts, $h / c_{0}=0.1$, $h_{s} / c_{0}=0.066, w / \lambda=0.033,0.067,0.13$.

\subsubsection{A simple analytical model to predict noise reductions}

Turbulent flow interacting with the profile sketched in figure 6.2 will induce variations in the pressure-difference (between pressure and suction sides) over the aerofoil. The largest pressure-differences principally occur along the leading edge whose strength and phase variation mostly determine the level of noise reduction in the far field. Previous work on single-wavelength profiles indicate that the dominant sources are located at the root region Kim et al. (2016); Chaitanya et al. (2017); Gea-Aguilera et al. (2017). The reason for this behaviour has been investigated by Turner and Kim (2017). They showed that the root of the serrated leading edge is the dominant noise source due to the presence of a secondary horseshoe-like vortex system generated by the serrated leading edge, which alters the upstream velocity field, thereby enhancing the source strength at the serration root. The noise reduction spectra shown below in section 6.3.4 suggests that dominant compact sources are also present in the slitted root profile but with localized sources located at either ends of the slit, as indicated by the green and red dots in figure 6.2.

Consider two compact sources located at the streamwise locations $x_{1}$ and $x_{1}+h_{s}$ with source strengths $q_{r}\left(x_{1}, \omega\right)$ and $q_{w}\left(x_{2}, \omega\right)$, as shown in figure 6.2. However, these source strengths have been shown on single wavelength serration to be solely a function of the non-dimensional frequency $\hat{\omega}=\omega h / U$, where $h$ is the overall serration height as demonstrated by Chaitanya et al. (2017). In the case of the slitted root, the source strengths $q_{r}$ and $q_{w}$ are now complicated functions of the slit geometry $q_{r}=q_{r}\left(\omega, h_{s}, h, w, \lambda\right)$ and $q_{w}=$ $q_{w}\left(\omega, h_{s}, h, w\right)$. As in the previous studies presented in Chapters 4 and 5, the path length differences between $q_{r}$ and $q_{w}$ to a far-field observer can be neglected since they are generally much closer together compared to an acoustic wavelength. The total radiated pressure is therefore proportional to the sum of source strengths with relative phase difference included,

$$
p(\omega) \propto q_{r}\left(x_{1}, \omega\right)+q_{w}\left(x_{1}+h_{s}, \omega\right) e^{-i \omega h_{s} / U} .
$$

In this chapter, similar to Chapter 5 , we make the assumption that the incoming turbulence is frozen, i.e., $v(\mathbf{x}, t)=v(\mathbf{x}-\mathbf{U} t)$, where $v$ is the velocity component normal to the aerofoil and $\mathbf{x}=(r, x)$ is any position in the flow. Further making the assumption that the turbulence 
is homogeneous, the space-time correlation function $R=E\left[v\left(x_{1}, t_{1}\right) v\left(x_{1}+h_{s}, t_{2}\right)\right]$ for the velocity $v$ between the two stream-wise positions $x_{1}$ and $x_{1}+h_{s}$ is of the form

$$
R\left(x_{1}, x_{1}+h_{s}, t_{1}, t_{2}\right)=\hat{R}\left(h_{s}-\left(t_{1}-t_{2}\right) U\right)
$$

where $\hat{R}\left(h_{s}-\left(t_{1}-t_{2}\right) U\right)$ is the spatial correlation function in the reference frame moving with the flow. A direct consequence of this frozen turbulence assumption is that the sources $q_{r}$ and $q_{w}$ at either ends of the narrow slit must be perfectly coherent since the turbulent eddies that excite them simply convect with the mean flow along the slit height $h_{s}$ as a frozen pattern of turbulence. The radiated sound power $W(\omega)$ is proportional to mean square pressure,

$$
W(\omega) \propto \overline{p^{2}}(\omega) \propto \mathbf{E}\left[p^{*}(\omega) p(\omega)\right]
$$

Substituting (6.1) into (7.8), the radiated sound power becomes,

$$
W(\omega) \propto\left[\overline{q_{r}^{2}}(\omega)+\overline{q_{w}^{2}}(\omega)+2 \overline{q_{r}(\omega) q_{w}^{*}(\omega)} \cos \left(\omega h_{s} / U\right)\right] .
$$

where $\overline{q_{r}^{2}}(\omega)$ and $\overline{q_{w}^{2}}(\omega)$ are the mean square source strengths at the frequency $\omega$ at the two locations either ends of the slit and $\overline{q_{r}(\omega) q_{w}^{*}(\omega)}$ represents the cross spectra between the two sources. The advantage of the slitted-root design is that these sources are perfectly coherent and hence the cross spectrum may be replaced by the product of the rms source strengths,

$$
\overline{q_{r}(\omega) q_{w}^{*}(\omega)}=\sqrt{\overline{q_{r}^{2}}(\omega) \overline{q_{w}^{2}}(\omega) .}
$$

The sound power radiation $W(\omega)$ from the slitted root serration may now be compared to the baseline un-slitted sawtooth profile of the same overall amplitude $W_{s}(\omega)$, which we assume is due to a single compact source at the root of the serration $q(\omega)$ (Chaitanya et al. (2017)) as sketched in figure 6.3. The additional sound power level reduction compared to the sawtooth baseline case may therefore be written as,

$\Delta P W L_{A}(\omega)=10 \log _{10}\left(\frac{W_{s}(\omega)}{W(\omega)}\right)=10 \log _{10}\left(\frac{\overline{q^{2}}(\omega)}{\overline{q_{r}^{2}}(\omega)+\overline{q_{w}^{2}}(\omega)+2 \sqrt{\overline{q_{r}^{2}}(\omega) \overline{q_{w}^{2}}(\omega)} \cos \left(\omega h_{s} / U\right)}\right)$

Overall sound power reductions provided by these slitted-root profiles therefore comprise the sum of two contributions. The first arises from the reduction in source strength along the oblique leading edge, which we shall denote as $\triangle \mathrm{PWL}_{S}$. The second arises solely from interference between the two coherent sources $q_{r}$ and $q_{w}$ at either ends of the slit, which we shall denote as $\Delta \mathrm{PWL}_{A}$. The overall sound power reduction may therefore be written as,

$$
\Delta \mathrm{PWL}(\omega)=\Delta \mathrm{PWL}_{S}(\omega)+\Delta \mathrm{PWL}_{A}(\omega) .
$$


Equation 6.6 for the additional noise reduction spectra makes clear that it is completely governed by two source strength ratios. One, $r(\omega)$, quantifies the relative strengths between the two sources at either ends of the slit,

$$
r^{2}(\omega)=\frac{\overline{q_{w}^{2}}(\omega)}{\overline{q_{r}^{2}}(\omega)} .
$$

The second, $s(\omega)$, quantifies the relative strengths between the source at the root location of the un-slitted profile and the sum of source strengths at either ends of the slit,

$$
s^{2}(\omega)=\frac{\overline{q^{2}}(\omega)}{\left(\sqrt{\overline{q_{r}^{2}}(\omega)}+\sqrt{\overline{q_{w}^{2}}(\omega)}\right)^{2}} .
$$

The final expression for the additional sound power level reduction in terms of the ratio of source strengths defined in Equations 6.8 and 6.9 is given by,

$$
\Delta P W L_{A}(\omega)=10 \log _{10}\left(\frac{s^{2}(\omega)}{1+\frac{2 r(\omega)}{(1+r(\omega))^{2}}\left(\cos \left(\pi \omega / \omega_{0}\right)-1\right)}\right)
$$

where,

$$
\omega_{0}=\pi U / h_{s}
$$

Equation 6.10 suggests that additional noise reductions obtained from the slitted root serration compared to the un-slitted profile are due to two factors. The first is due to a reduction in the source strengths between the un-slitted $q^{2}(\omega)$ and the slitted profiles $\left(\sqrt{\overline{q_{r}^{2}}(\omega)}+\right.$ $\sqrt{\left.\overline{q_{w}^{2}}(\omega)\right)^{2}}$ as accounted for by the ratio $s(\omega)$. The second factor is due to interference between the two source strengths at either ends of slit whose effect is quantified by the denominator in Equation 6.10. This term goes to zero, and hence perfect noise cancellation is predicted, when the source strengths at the ends of the slit at the frequency $\omega_{0}$ are equal, i.e., $r\left(\omega_{0}\right)=1$, and radiate exactly $180^{\circ}$ out of phase. Here, we refer to $\omega_{0}$ given by Equation 6.11 as the tuned frequency of the slitted-root profile.

\subsubsection{Limiting case}

As discussed earlier, we are concerned with the two limiting cases of slitted-root geometry. One occurs for very small slit width for which the source strength $q_{w}(\omega)$ at the downstream end of the slit tends to zero and hence, $r(\omega) \rightarrow 0$. The other is when the slitted-root parameters are chosen so that the source strengths at either ends of the slit are equal and hence $r(\omega) \rightarrow 1$. We now consider each case in turn. 


\section{Slit width tends to zero}

In the case of $r(\omega) \rightarrow 0$, Equation 6.10 becomes,

$$
\Delta P W L_{A}(\omega)=20 \log _{10}(s(\omega)),
$$

In this limiting case the sound power reduction is therefore determined solely by $s(\omega)$ the ratio between the source strengths at the root of the baseline sawtooth to that of the total source strengths of the slitted-root. An unexpected finding of this work is that, in this limiting case, at frequencies below the tuned frequency, noise reductions are found to be almost identical to those of a conventional sawtooth serration of the same peak-to root distance $2 h$. The ratio of source strengths between the slitted and un-slitted profiles must therefore be equal in the low frequency limit, i.e, $s(\omega) \rightarrow 1$ for $\omega / \omega_{0} \rightarrow 0$. For very narrow slits, $q_{w}(\omega)$ must be negligible and hence,

$$
\overline{q_{w}^{2}(\omega)} \rightarrow \overline{q^{2}(\omega)}, \quad\left(\omega / \omega_{0} \rightarrow 0, r \rightarrow 0\right) .
$$

Very narrow slits therefore provide near identical noise reductions to the conventional sawtooth (and single wavelength) serration profiles of the same amplitude. This finding has potentially significant benefits for the design of low noise aerofoils since introducing narrow slits is a much smaller modification to the aerofoil than conventional sawtooth serrations of the same amplitude.

\section{Optimum slit geometry}

The second limiting case of interest here is when the source strengths at either ends of the slit are exactly equal at the tunned frequency, i.e., $r\left(\omega_{0}\right)=1$, which can be obtained through optimal choice of slitted root geometry. In this case, the noise reductions from Equation 6.10 are given by,

$$
\Delta P W L_{A}(\omega)=20 \log _{10}\left(s(\omega) \sec \left(\pi \omega / 2 \omega_{0}\right)\right) .
$$

Noise reductions are now solely controlled by the frequency $\omega$ in relation to the tuned frequency $\omega_{0}$. Thus, by simply introducing narrow slits onto a leading edge profile, significantly enhanced noise reductions can be obtained compared to un-slitted profiles of the same amplitude. In the next section, the experimental setup is described which allows the proposed noise reduction mechanisms to be validated experimentally. Subsequent sections will present levels of noise reduction substantially higher (by up to $11 \mathrm{~dB}$ ) than can be achieved with conventional profiles of the same amplitude.

\subsubsection{Acoustic performance on flat plate}

We start by demonstrating the superior noise reduction performance of slitted-root serrations compared to the more conventional un-slitted profiles of the same amplitude. Figure 6.5 shows the sound power reduction spectra plotted against non-dimensional frequency $f h / U$ for a typical slitted-root serration with serration amplitude $h / c_{0}=0.167$, slit width $w / \lambda=0.133$, slit height $h_{s} / 2 h=0.64$ at the four flow speeds of $20,40,60$ and $80 \mathrm{~m} / \mathrm{s}$. Also 


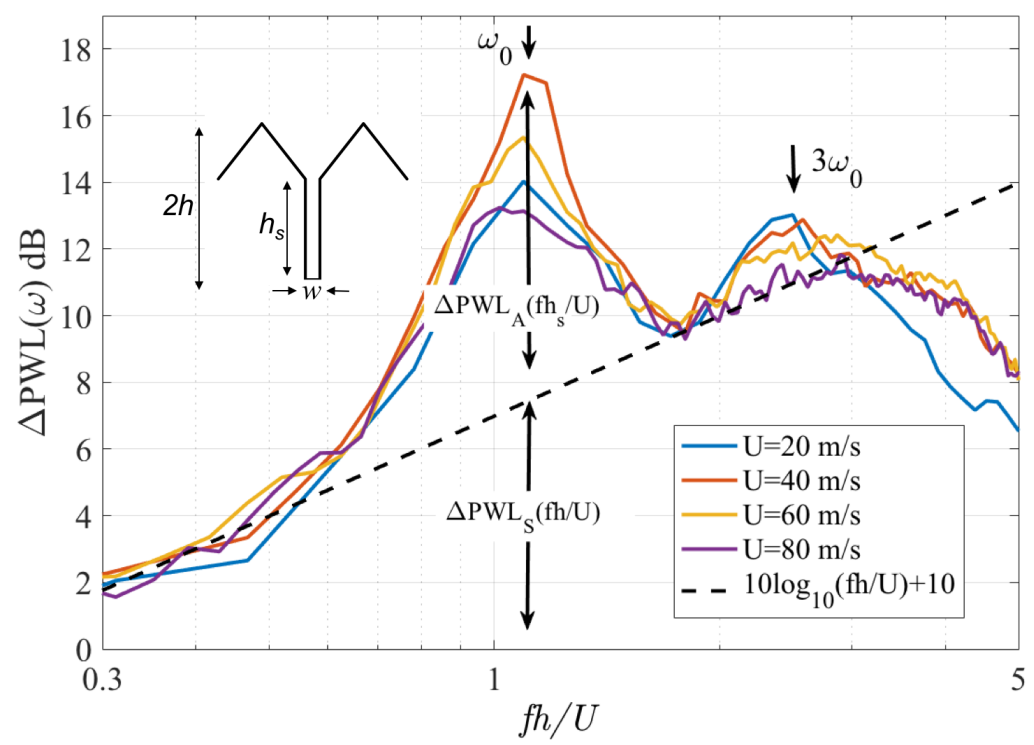

FIGURE 6.5: Influence of jet velocity on noise reductions of slitted-root serrations of slit width $w / \lambda=0.133$, slit amplitude $h_{s} / 2 h=0.64$ and serration amplitude $h / c_{0}=0.167$.

shown is the line $10 \log _{10}(f h / U)+10$ representing the optimum sound power reduction achievable for a single-wavelength serration at the optimum wavelength.

The noise reduction spectra due to the slitted root serration can be seen to peak at a frequency very close to the tuned frequency $\omega_{0}$ marked on this figure as a vertical arrow. The noise reduction at this peak frequency is substantially higher by about $10 \mathrm{~dB}$ than the corresponding level of the optimum sawtooth geometry of the same amplitude. A second peak in the noise reduction spectra is also apparent at approximately three times the tuned frequency, also marked on this figure. Overall, the slitted-root profile considerably outperforms the single sawtooth profile of the same amplitude. The characteristics of the noise reduction spectra are entirely consistent with the hypothesis of destructive interference between two dominant compact source regions of roughly equal strength at either ends of the slit, as sketched in Figure 6.2. Almost perfect collapse of the noise reduction spectra at the four different flow speeds are obtained with greatest deviations occurring at the peak frequency, demonstrating that the ratio between the serration characteristic length and the hydrodynamic wavelength $U / f$ is the important parameter in determining the level of noise reductions.

In the next section the sensitivity of noise reduction to slit width $w$ is investigated, with particular emphasis on highlighting the different noise reduction mechanisms involved in the limit of very small slit width, $w \rightarrow 0$, and the optimum slit width when $r(\omega) \rightarrow 1$.

\subsubsection{Influence of $w$ on additional noise reductions}

Figure 6.6 shows the spectra of noise reductions $\Delta P W L_{A}(\omega)$ additional to the noise reductions achievable with a single-sawtooth serration of the same amplitude and optimum wavelength. The spectra are now plotted against non-dimensional frequency $\omega / \omega_{0}$ to make explicit the role of interference in the noise reduction mechanism. The results from 7 different slit widths $w / \lambda$ of $0.033,0.067,0.1,0.13,0.167,0.2$ and 0.233 are presented at a fixed slit 


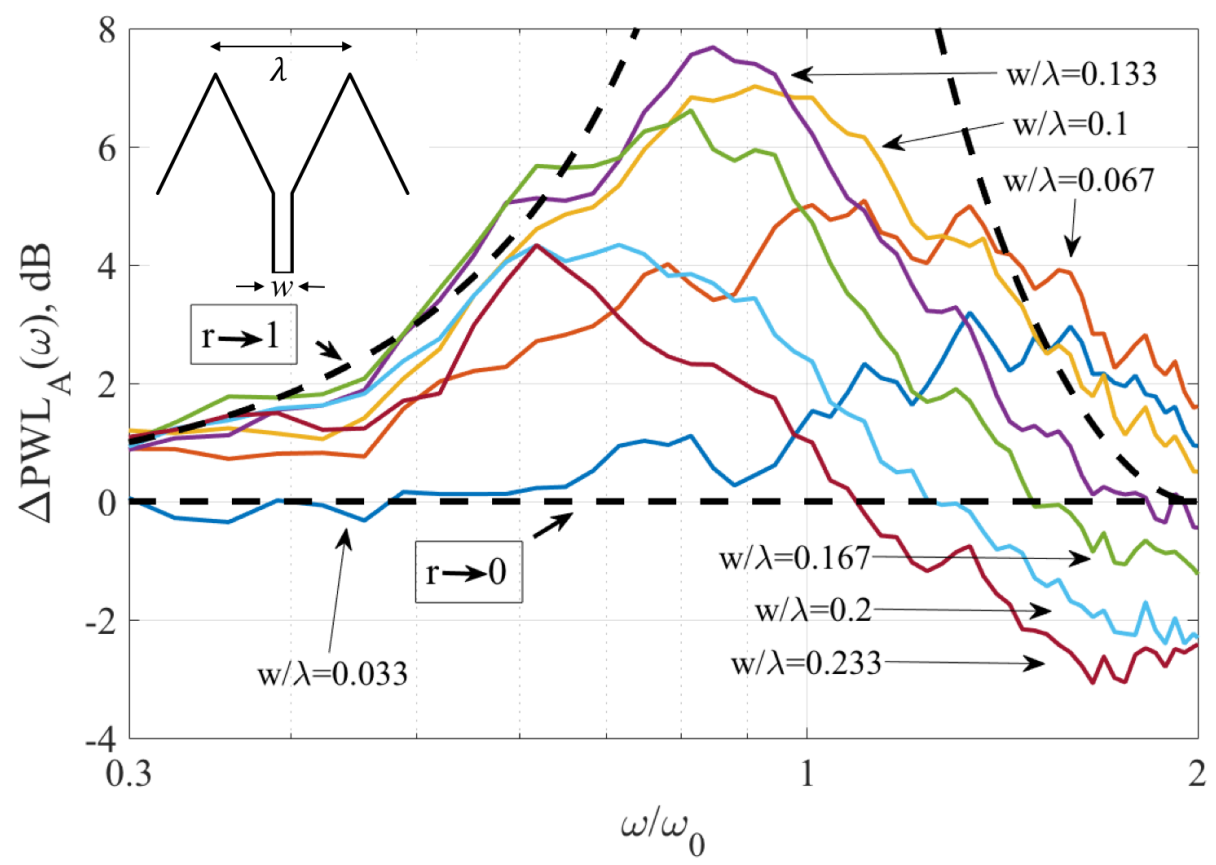

FIGURE 6.6: Additional sound power reduction comparisons for a slitted-root serration for varying slit width $w / \lambda$ at fixed slit amplitude $h_{s} / c_{0}=0.133$, serration amplitude $\left(h / c_{0}=0.1\right)$ at jet velocity of $60 \mathrm{~m} / \mathrm{s}$.

height $h_{s} / c_{0}=0.133$ and flow speed of $60 \mathrm{~m} / \mathrm{s}$. These additional noise reduction spectra were obtained by subtracting the sound power level spectra due to a slitted-root serration from that due to the un-slitted-sawtooth serration of the same peak-to-root amplitude. Also shown in this figure are Equations $20 \log _{10} s(\omega)$ and $20 \log _{10}\left(s(\omega) \sec \left(\pi \omega / 2 \omega_{0}\right)\right)$ representing the theoretical additional noise reduction spectra for the two limiting cases of narrow slit and optimum slit width respectively, where we have used a value of $s(\omega)=1$. We emphasize that, in general, $s(\omega)$ is not known. However, $s(\omega)=1$ was chosen for consistency with the results of figures 6.6 and 6.5, which both indicate that, for all widths under investigation, the slitted and un-slitted serrations provide nearly identical noise reductions, and hence we can infer that $s(\omega) \rightarrow 1$ in the low frequency limit.

The results in Figure 6.6 provide an overview of the advantages of introducing slits at the serration roots whilst maintaining the same overall amplitude.

The noise reduction spectra in this figure may be summarized as follows:

- For all slit widths $w$ investigated, levels of noise reduction always exceed those of the un-slitted sawtooth serrations of the same amplitude. The exception is at high frequencies where its performance is slightly worse compared to the sawtooth profile (by a few $\mathrm{dB}$ ). We emphasize that this geometry still provides noise reductions compared to a straight leading edge.

- For the smallest slit width $w / \lambda=0.033(w=0.5 \mathrm{~mm})$ under investigation, almost zero additional noise reductions are observed suggesting that introducing extremely narrow slits at the roots of the serrations provides the same noise reduction as the sawtooth serration of the same amplitude. The obvious advantage of the new profile is that it represents a much smaller modification to the leading edge which has benefits in terms of structural integrity and aerodynamic performance. 


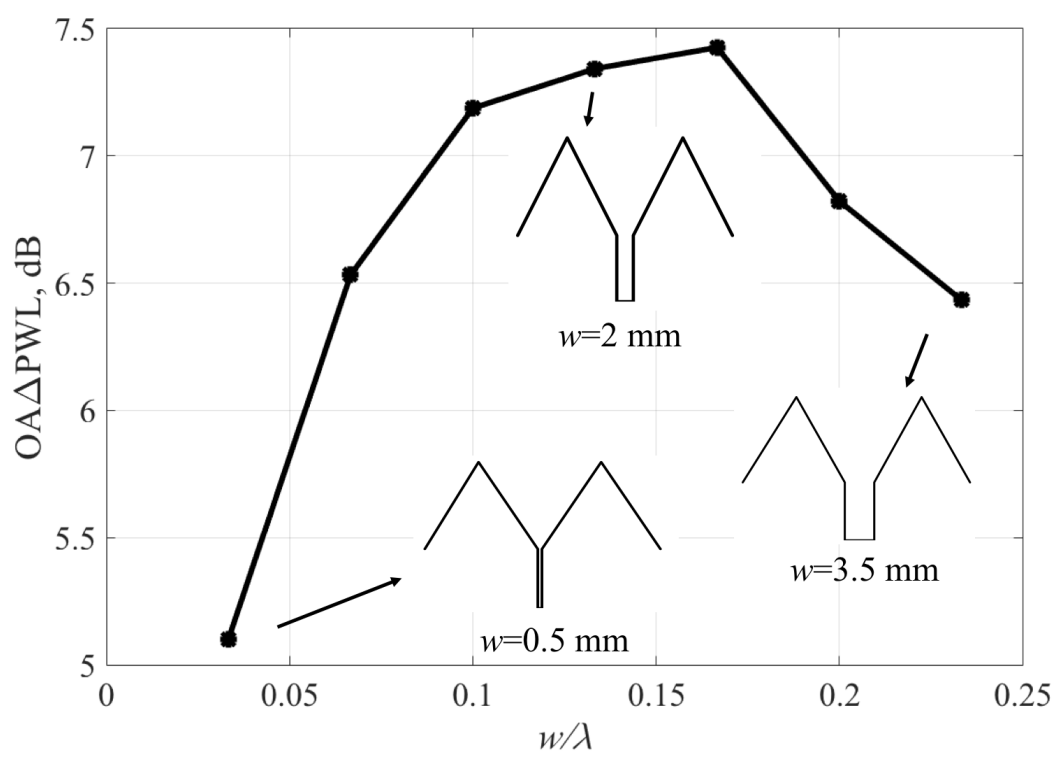

FIGURE 6.7: OAPWL versus $w / \lambda$ for a slitted-root serration for varying slit width $w / \lambda$ at fixed slit amplitude $h_{s} / c_{0}=0.133$, serration amplitude $\left(h / c_{0}=\right.$ 0.1 ) at jet velocity of $60 \mathrm{~m} / \mathrm{s}$.

- As the slit width is progressively increased the levels of noise reduction increase, reaching maximum values at $w / \lambda=0.133$. At slit widths greater than this value, levels of additional noise reduction are observed to reduce. This observation implies the existence of an optimum slit width whose additional noise reduction spectra match very closely the theoretical curve $20 \log _{10}\left(s(\omega) \sec \left(\pi \omega / 2 \omega_{0}\right)\right)$ predicted based on interference between two sources of identical strength separated by a distance equal to the slit height $h_{s}$. This observation validates the noise reduction mechanism proposed in Section 6.3.2. As $s(\omega)=1$ is assumed in this prediction, this theoretical curve therefore represents the upper limit on the noise reduction that can be obtained through the use of slitted-root serrations.

- The two theoretical spectra $20 \log _{10} s(\omega)$ and $20 \log _{10}\left(s(\omega) \sec \left(\pi \omega / 2 \omega_{0}\right)\right)$ therefore represent the lower and upper limits on the noise reductions that may be achieved using slitted profiles additional to the noise reduction on un-slitted profiles. All additional noise spectra therefore fall within these two limits.

Figure 6.7 is a plot of the overall sound power reductions integrated over the non-dimensional frequency range of $\omega / \omega_{0}$ of between 0.3 and 3 plotted against non-dimensional slit width $w / \lambda$. It provides explicit evidence for the existence of an optimum slit width, which in this case is $w / \lambda=0.167$ representing a slit width of just $2.5 \mathrm{~mm}$. However, increasing or reducing this slit width by just $0.5 \mathrm{~mm}$ causes a significant decrease in noise reductions, indicating that the source balance between $q_{r}$ and $q_{w}$ is highly dependent on slit width, as we shall shown section 6.3 .7 below.

\subsubsection{Influence of $h_{s}$ on additional noise reductions}

In this section we investigate the influence of the slit height $h_{s}$ on the noise reductions. The additional noise reduction spectra $\triangle P W L_{A}$ are plotted against non-dimensional frequency $\omega / \omega_{0}$ in Figure 6.8 for eight different slit heights $h_{s} / 2 h$ of $0.2,0.4,0.6,0.64,0.7,0.8,0.9$, 


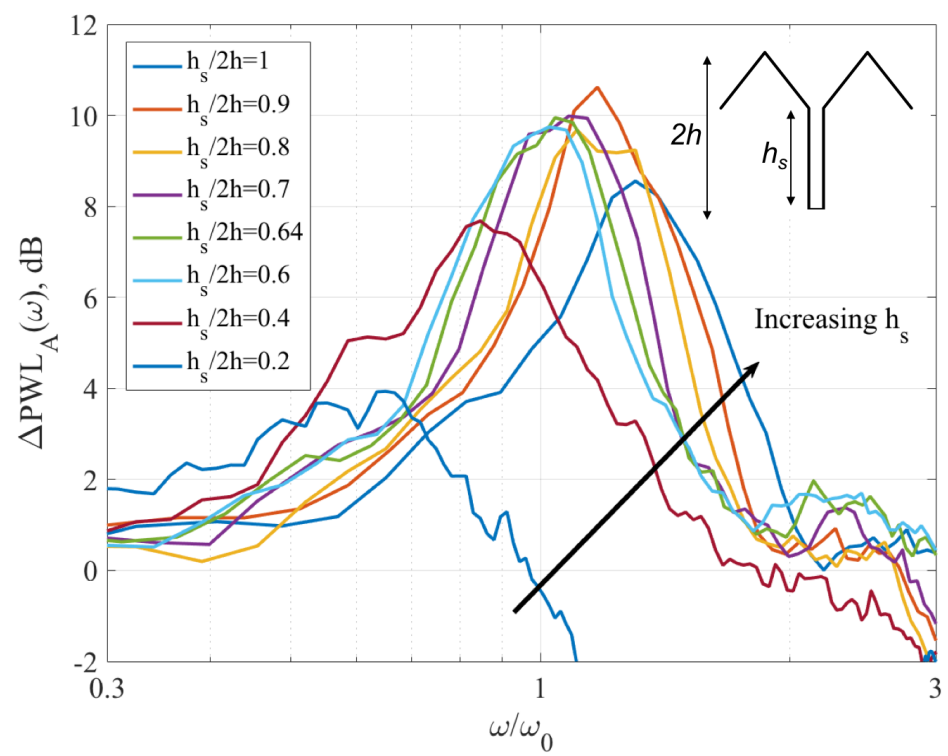

FIGURE 6.8: Additional noise reductions $\triangle P W L_{A}$ versus non-dimensional frequency $\omega / \omega_{0}$ for a serration ampltiude $h / c_{0}=0.167$, wavelength $\lambda / c_{0}=0.1$ and jet velocity $U=60 \mathrm{~m} / \mathrm{s}$.

1 (obtained by adjusting the inclination angle of the oblique edge). Note that $h_{s} / 2 h=1$ represents the limiting case of only slits without a sawtooth profile, as sketched in Figure 6.8. In this figure the overall amplitude $2 h / c_{0}$ is kept constant at 0.33 , the slit width is kept constant at the optimum value identified in figure 6.6 of $w / \lambda=0.133$ and the serration wavelength kept constant at $\lambda / c_{0}=0.1$ corresponding to four times the turbulence integral length-scale.

The peaks in the noise reduction spectra in Figure 6.8 of maximum noise reduction all occur close to the tuned frequency of $\omega / \omega_{0} \approx 1$.

A clear trend in the noise reduction spectra is observed. As the slit height $h_{s}$ is increased the peak frequency $\omega / \omega_{0}$ of maximum noise reduction approaches, from below, a value of approximately 1.25 while the level of maximum additional reduction increases, reaching a level of approximately $11 \mathrm{~dB}$ (compared to a conventional sawtooth profile of the same amplitude). Based on the model presented in section 6.3.2, this finding suggests that, as $h_{s}$ is decreased, the sources at either ends of the slit become more equal in strength and their effective separation distance approaches a limiting value corresponding to an effective slit height of $0.8 h_{s}$ (corresponding to a peak frequency of $\omega / \omega_{0}=1.25$ ).

To quantify the sensitivity of the noise reduction performance to slit height, the overall sound power reductions were calculated over the non-dimensional frequency range of $f h / U$ of between 0.15 and 2 and plotted against $h_{s} / 2 h$ in Figure 6.9.

For this combination of slit width, wavelength and frequency bandwidth, the overall noise reduction can be observed to increase sharply with increasing slit height up to a value of about $h_{s} / 2 h=0.6$. Above this value, overall noise reductions appear to fluctuate by nearly $1 \mathrm{~dB}$ even though the frequency of maximum noise reduction reduces as $1 / h_{s}$, according to Eq. 6.11. The extreme value $h_{s} / 2 h=1$ in this figure corresponds to the special case in which only slits are present at the leading edge with no sawtooth roots. Simply adding narrow slits (just $2 \mathrm{~mm}$ in this case) is therefore capable of providing considerably greater noise reductions than conventional single-wavelength or sawtooth profiles alone. The effectiveness of 


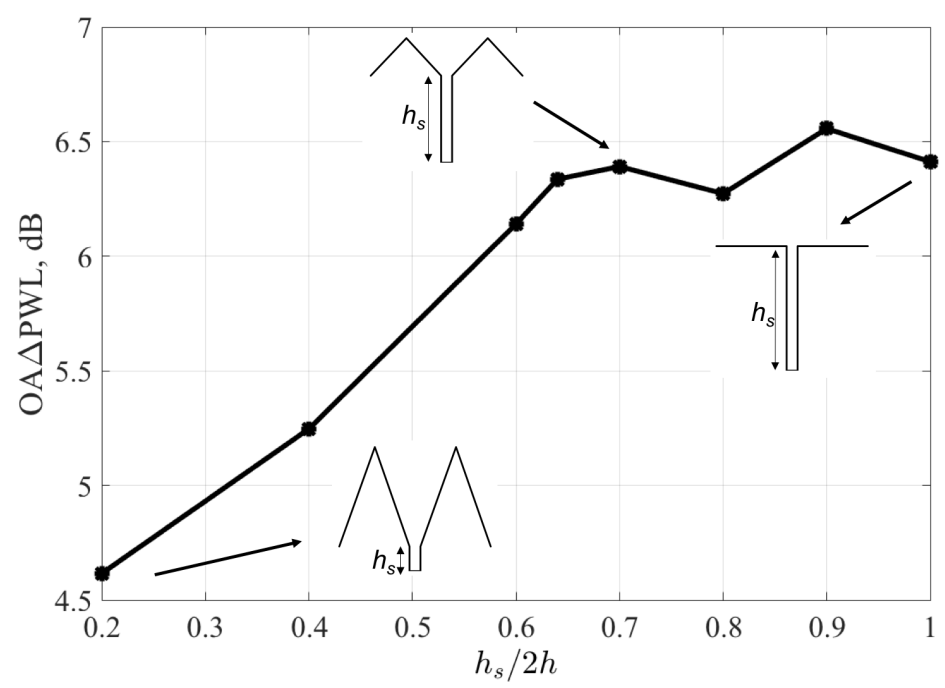

FIGURE 6.9: Overall sound power reductions $O A \Delta P W L_{A}$ versus $h_{s} / 2 h$ for a serration amplitude $h / c_{0}=0.167$, wavelength $\lambda / c_{0}=0.1$ and jet velocity $U=60$ $\mathrm{m} / \mathrm{s}$.

introducing slits to a 5\% thick 3D aerofoil typical of an Outlet Guide vanes will be examined in section 6.4.4.

\subsubsection{Comparison between predicted and measured additional noise reductions}

To assess the validity of the noise reduction mechanism described by Equation 6.10 in Section 6.3.2 for predicting the noise reductions additional to the baseline (un-slitted sawtooth geometry), predictions from this equation are compared to the measured additional noise reduction spectra for the three different slit heights of $h_{s} / 2 h=0.4,0.7$ and 1 . In this comparison the slitted root profile has a peak-to-root distance $2 h / c_{0}=0.33$, a slit width of $w / \lambda=0.13$, and at a flow speed equal to $60 \mathrm{~m} / \mathrm{s}$. In figure 6.10 the measured noise reduction spectra plotted against $\omega / \omega_{0}$ is compared against the predicted spectra with assumed values of $s(\omega)=1$ for the three values of $r(\omega)$ of $0.4,0.53,0.45$ chosen to provide best fit to the measured data for each value of $h_{s}$ over the frequency range of interest. Note that, for simplicity, we have assumed that $r(\omega)$ is independent of frequency. These simulations also assume a value of $s(\omega)=1$, which we know to be correct only in the low frequency limit. However, its precise value at higher frequencies is unknown and is therefore a source of error in the noise reduction predictions. Finally, the slit height $h_{s}$ in the expression for the tuned frequency $\omega_{0}=\pi U / h_{s}$ is adjusted by no more than $20 \%$ of the physical value to give best fit to the frequency of maximum noise reduction. This slit height $h_{s}$ may therefore be regarded as the effective slit height $h_{s}^{*}$ corresponding to the streamwise separation distance between the two sources at either ends of the slit.

Very good levels of agreement are obtained over the entire frequency range with the measured noise reduction spectra by the simple model obtained by adjusting just the two parameters of $\omega_{0}$ and $r(\omega)$ (where $\omega / \omega_{0} \approx 1$ ), whilst assuming $s(\omega)=1$.

The key to obtaining good levels of noise reduction by the use of these profiles is to ensure that the source strengths at either ends of the slit are as equal as possible at the tuned frequency $\omega_{0}$. It is therefore important to understand how the ratio of source strengths $r(\omega)$ 


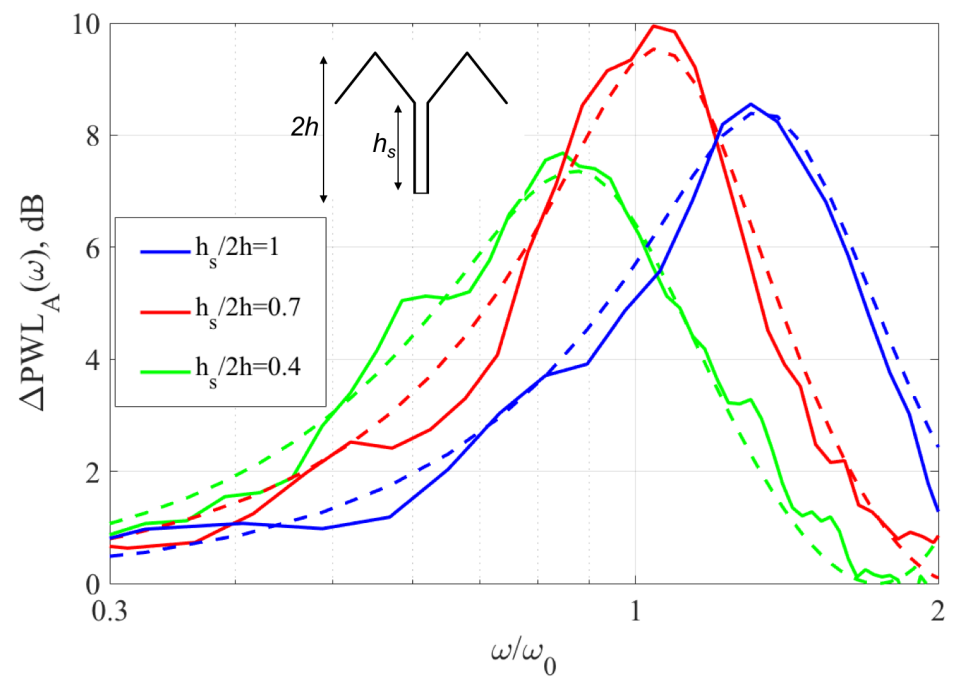

FIGURE 6.10: Comparison between analytic and experimental noise reductions for slitted-root serrations of $h_{s} / c_{0}=0.213, w / \lambda=0.132 h / c_{0}=0.33$, $\lambda / \Lambda_{o}=4$ at jet velocity $\mathrm{U}=60 \mathrm{~m} / \mathrm{s}$.

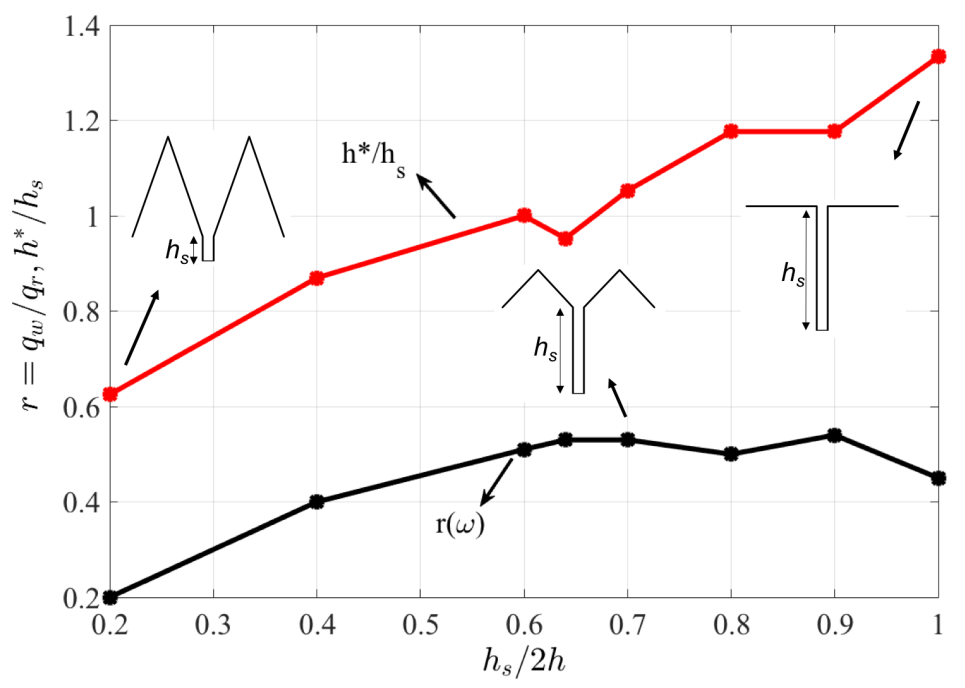

FIGURE 6.11: Source strength $r$ versus $h_{s} / 2 h$ (Black line) and ratio of effective slit height to actual slit height calculated from the measured data (Red line) . 


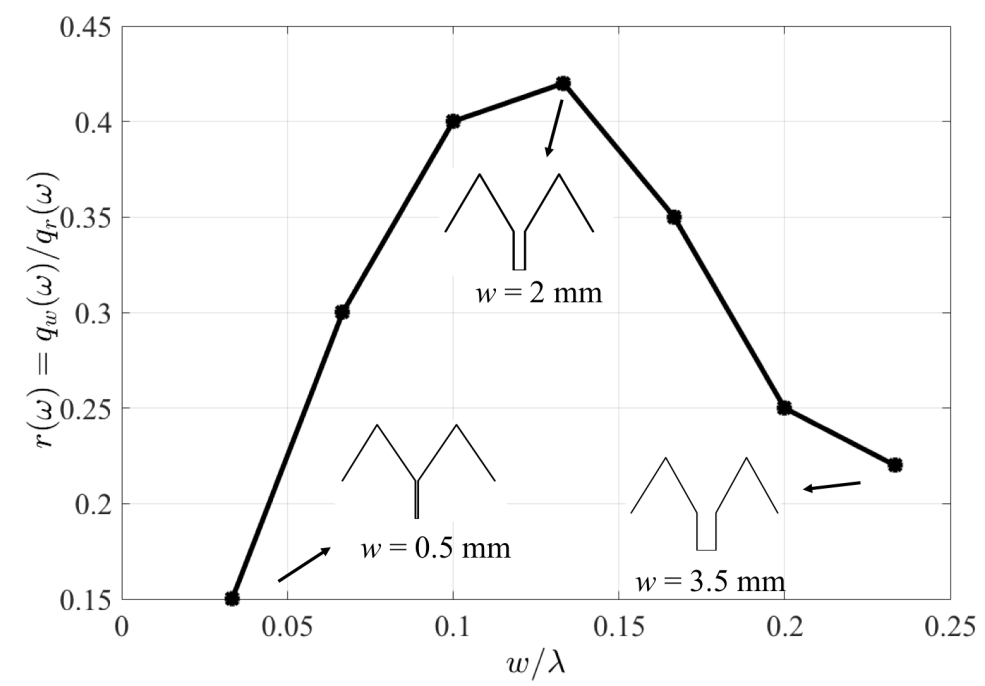

FIGURE 6.12: Source strength $r$ versus $w / \lambda$ calculated from the measured data.

varies with both $h_{s}$ and $w$. An estimate for $r(\omega)$ for any slitted-root geometry may be obtained from the value which most closely matches the predicted noise reduction spectra to the measured spectra.

Figure 6.11 shows the variation of $r(\omega)$ (black line) and $h_{s}^{*}$ (red line) with slit height obtained by following this procedure. This figure suggests that $r(\omega)$ tends from above towards a maximum value of 0.55 as $h_{s}$ increases. Unsurprisingly, the variation of $r$ with $h_{s}$ follows a similar variation to the overall sound power variation plotted in Figure 6.9, since noise reductions are predicted to increase as $r$ tends to 1 . We emphasize that the estimate of $r(\omega)$ in Figure 6.11 are only approximate values owing to the erroneous assumption that $s(\omega)=1$ and that $r(\omega)$ is frequency independent. Additional sources of error are also introduced by the assumption that the turbulence is frozen as it convects between the serration peak and the root where in practice some de-correlation of the turbulent flow will occur. Nevertheless, Figure 6.11 provides useful information about the design of the slitted profiles for maximum noise reduction.

Finally, we consider the variation in source strength ratio $r(\omega)$ with slit width, which we plot in figure 6.12 against slit width $w / \lambda$ normalized on $\lambda$. The results for 7 values of slit width are plotted for $h_{s} / 2 h=0.4$. Unlike with the variation in $h_{s}$, a clear optimum value of $w / \lambda=0.13$ in slit width (corresponding to an actual width of just $2 \mathrm{~mm}$ ) is observed.

\subsubsection{Sound Power Spectra comparison for 3D aerofoils}

We now investigate the noise effectiveness of the control principle demonstrated above for flat plates to 3D aerofoils. A slitted-root sawtooth profile was introduced onto a NACA65 aerofoil by applying the design method of Eq. 5.26 in section 5.9. The slitted-root sawtooth geometry was chosen to have serration amplitude of $h_{s} / c_{0}=0.1$ and optimum serration wavelength $\lambda=4 \Lambda_{t}$ and optimum slit width $w / \lambda=0.13$, as discussed in Section 6.3.4. A $3 \mathrm{D}$ view of the slitted-root geometry is shown in Figure 6.13. The sound power reduction spectra for the 3D aerofoil are plotted in Figure 6.14 versus $f(2 h) / U$ together with the corresponding power reduction obtained for the $3 \mathrm{D}$ baseline serration without slits. Also shown 


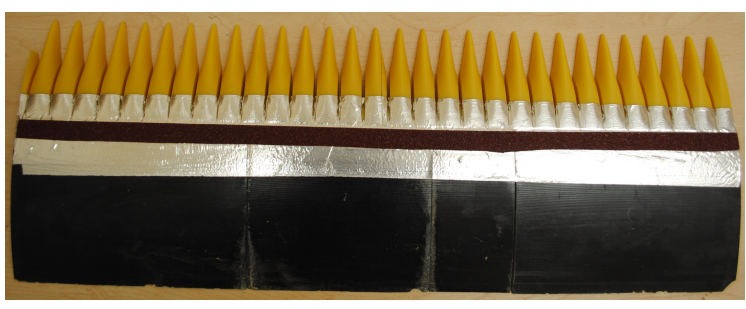

(a) Slitted-root serrated aerofoil.

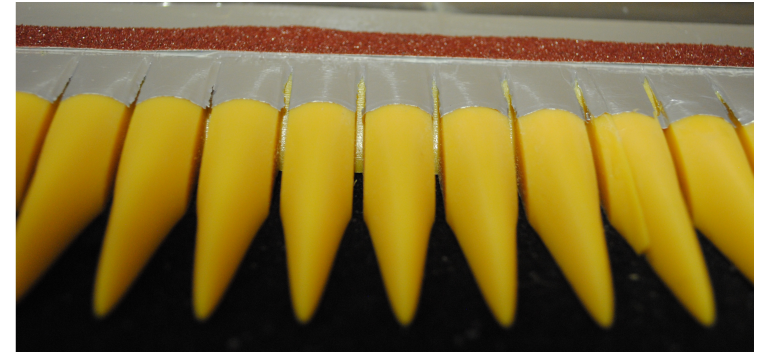

(b) Details of slitted-root geometry.

FIGURE 6.13: Top view of Slitted-root 3D geometry fabricated using 3D printer.

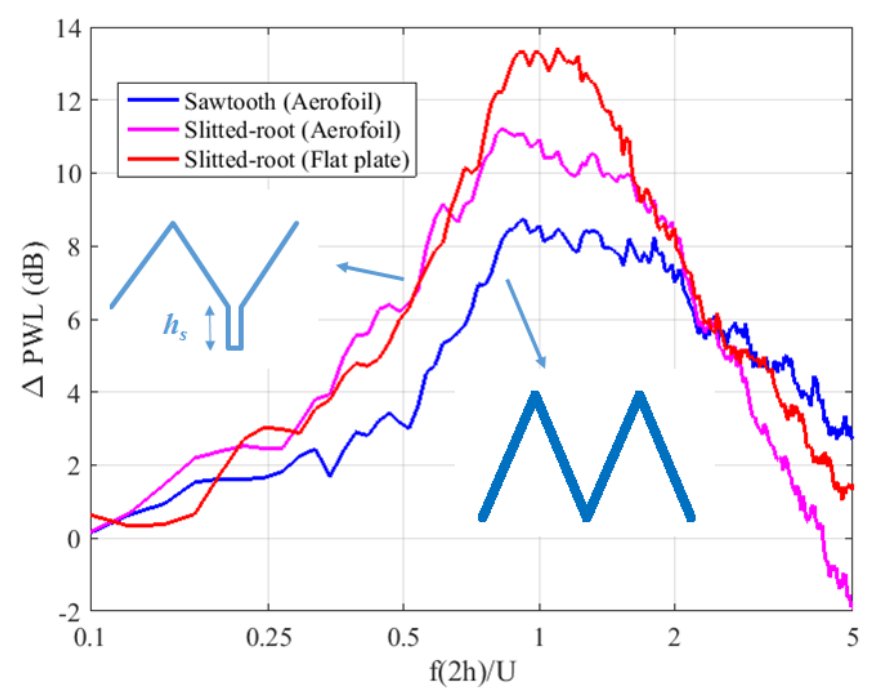

FIGURE 6.14: Sound power reduction comparisons for a slitted-root serration of amplitude $\left(h / c_{0}=0.1\right), w / \lambda=0.13, h_{s} / c_{0}=0.1$ with sawtooth at jet velocity of $60 \mathrm{~m} / \mathrm{s}$.

is the power reduction spectra for the slitted sawtooth profile cut into the flat plate discussed above.

Levels of noise reduction obtained for the slitted-root sawtooth on the 3D aerofoil are almost identical to that for the flat plate at frequencies below about $2 f h / U \approx 0.8(1 \mathrm{kHz})$. Above this frequency, 3D flow effects can be seen to degrade the performance compared to a flat plate. However, noise reductions for the 3D slitted sawtooth are better than those for the non-slitted sawtooth case except at high frequencies thus validating the control principle even for thick aerofoils. Negative noise reductions at high frequencies indicate a noise increase, which may be caused by a Helmholtz resonance formed in the cavity between the two parallel slides of the slits. An alternative slitted geometry is considered below in section 6.5, which is expected to reduce this effect.

\subsection{Slitted profiles}

In this section, we considered a limiting case of slitted-root serration $\left(h_{s} / 2 h=1\right)$ which provide significant noise reductions at low frequencies, as the maximum noise reduction 


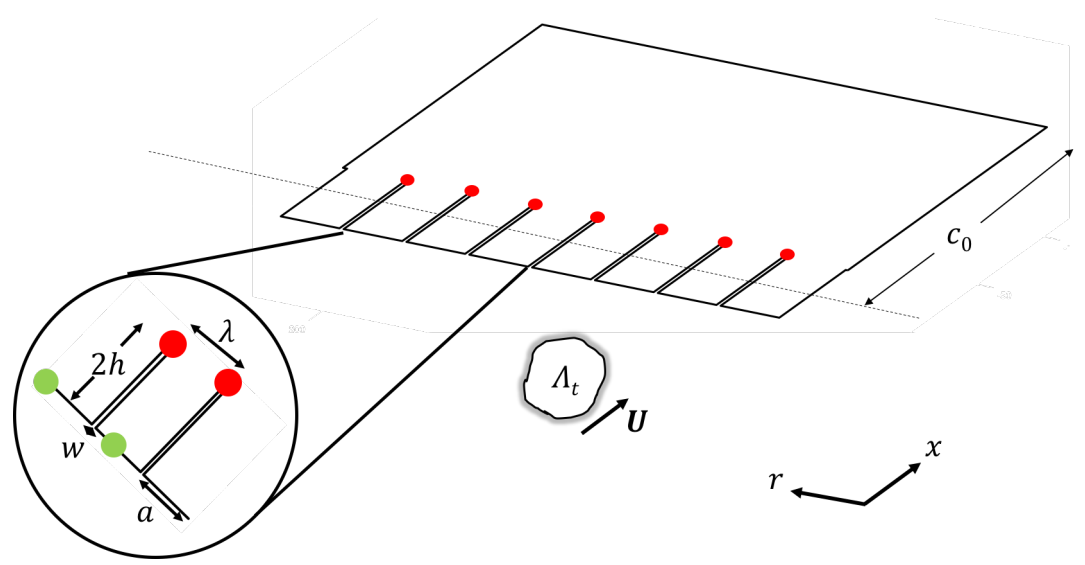

FIGURE 6.15: A sketch of the slitted-root leading edge serration.

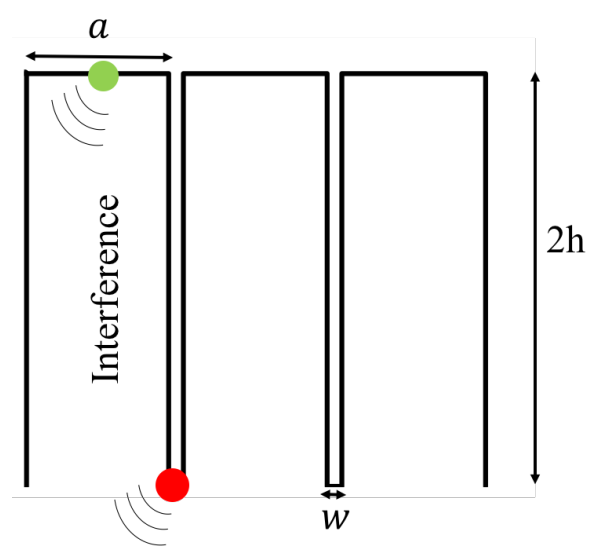

FIGURE 6.16: A schematic representation of slitted leading edge profiles.

reduces as $1 / h_{s}$. This is the best possible design which is capable of providing maximum low frequency noise reductions. A systematic parametric study on the slit dimensions $a$ and $w$ (see figure 6.16) has been performed to identify the optimum slit dimension ratio for maximum noise reductions.

\subsubsection{Geometry}

The Slitted leading edge profile investigated in this section involves introducing narrow slits at leading edge of the aerofoil, as sketched in figure 6.15.

The principal dimensions of the slitted profile is sketched in figure 6.16. For sufficiently large slit widths $w$, the peak in the noise reduction spectra shown in Section 6.4.2 suggests that the location of the dominant sources (indicated as red and green dots) are located at either ends of the slit separated in the streamwise direction by $2 h$. The overall distance between the peak and the end of the slit is $2 h$, with the pattern repeating with periodicity (wavelength) $\lambda=a+w$. 


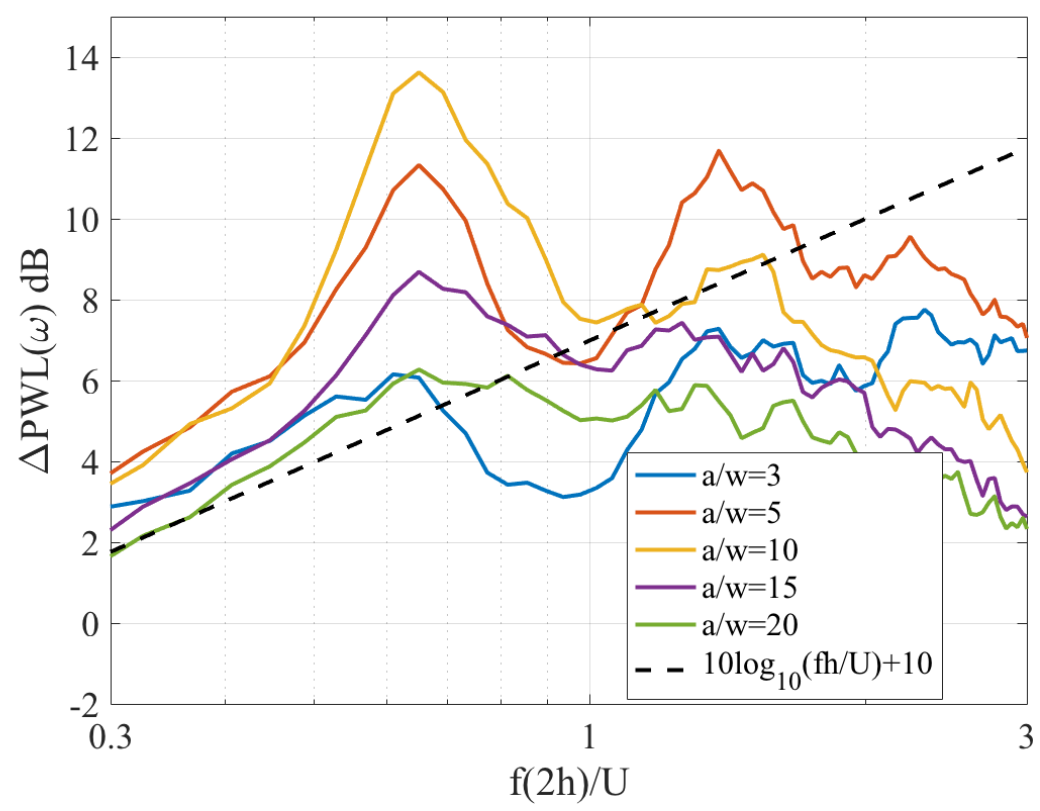

FIGURE 6.17: sound power reduction comparisons for a slitted serration for varying slit distance $a$ at fixed slit width $w=1 \mathrm{~mm}$, at jet velocity of $60 \mathrm{~m} / \mathrm{s}$.

\subsubsection{Influence of $a$ and $w$ on noise reductions}

Two principle dimensions of the slitted profiles $a$ and $w$ are systematically varied for different combinations of $w$ varying from $0.5 \mathrm{~mm}$ to $10 \mathrm{~mm}$ and $a$ varying from $3 \mathrm{~mm}$ to 60 $\mathrm{mm}$ respectively. A total of 40 cases are considered in this study. Figure 6.17 shows the comparison of sound power reductions spectra for 5 different width ratios $a / w$ of $3,5,10,15$ and 20 at a fixed slit width of $1 \mathrm{~mm}$ and a jet velocity of $60 \mathrm{~m} / \mathrm{s}$. A well defined optimum $a / w$ of 10 is observed for a slit width of $1 \mathrm{~mm}$. It is believed from earlier model proposed in section 6.3.2, for this combination of $a$ and $w$, the source strength ratio $r(\omega)$ must be close to 1 i.e., the condition when the two compact source are equal such that they interference at tuned frequency $\omega_{0}=\pi U / 2 h$.

A total of 40 slitted profiles of varying slit widths $a$ and $w$, were investigated to explore the sensitivity on noise reductions to these parameters. The below table 6.1 summaries the optimum dimension ratio $a / w$ for 7 different values of slit width $w$ varying from $0.5 \mathrm{~mm}$ to $10 \mathrm{~mm}$.

\begin{tabular}{c|c|c}
$\mathrm{w}(\mathrm{mm})$ & $\mathrm{a}(\mathrm{mm})$ & $\mathrm{a} / \mathrm{w}$ \\
\hline 0.5 & 5 & 10 \\
\hline 1 & 10 & 10 \\
\hline 2 & 15 & 7.5 \\
\hline 3 & 20 & 6.66 \\
\hline 4 & 25 & 6.25 \\
\hline 5 & 30 & 6 \\
\hline 10 & 40 & 4 \\
\hline
\end{tabular}

TABLE 6.1: Optimum $a / w$ ratio for various slit widths.

The reason for the decrease in the $a / w$ ratio with increase in slit width $w$ is currently not clear. From the previous chapters 4 and 5, the incoming turbulent length scale is also key factor to 


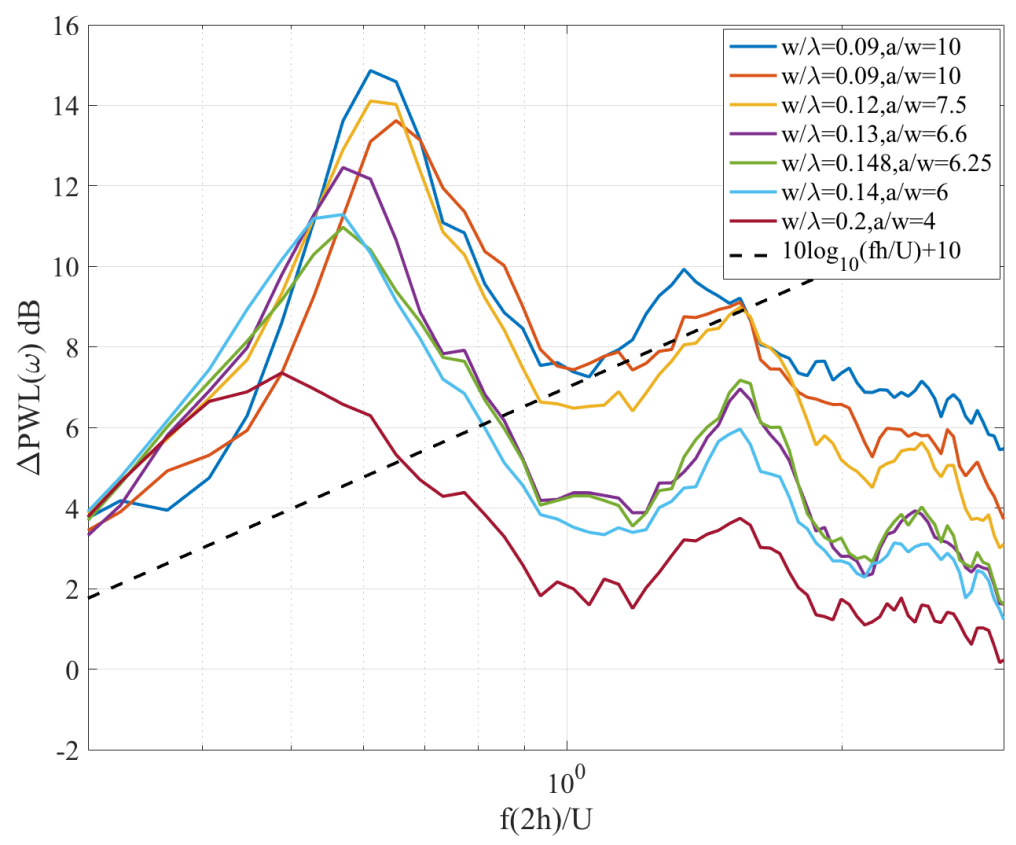

FIGURE 6.18: Comparison of sound power reductions spectra at optimum $a / w$ ratio for 7 different slit widths of $0.5,1,2,3,4,5,10 \mathrm{~mm}$ at a fixed jet velocity of $60 \mathrm{~m} / \mathrm{s}$.

determine this source balance. Detailed numerical investigation is required to explain this optimum $a / w$ ratio behaviour.

\subsubsection{Optimum sound power redictions}

Figure 6.18 shows the comparison of sound power reductions spectra at optimum $a / w$ ratio for 7 different slit widths of $0.5,1,2,3,4,5,10 \mathrm{~mm}$ (as tabulated in table 6.1) respectively at a fixed jet velocity of $60 \mathrm{~m} / \mathrm{s}$. The maximum noise reductions spectra given by $10 \log _{10}(f h / U)+10$ for single-wavelength profiles, is also shown in this figure as the dashed line.

An optimum slit width $w / \lambda=0.12$ has been identified and this design has be implemented on 3D OGV to demonstrate the effectiveness on 3D aerofoils. From Figure 6.18, with the increase of slit width $w / \lambda$, we observe an decrease in performance of slitted profiles. The reason for this behaviour is currently not know but more likely due to influence of correlation length which is function of incoming integral length scale of turbulence.

\subsubsection{Sound Power Spectra comparison for 3D aerofoils}

A symmetric thin aerofoil of 5\% thickness (Typical of an Outlet Guide Vane) of $0.15 \mathrm{~m}$ chord was also investigated in this study. To maximize the noise reduction at low frequencies, only narrow slits are introduced at the leading edge, i.e., with no sawtooth profile. The slit amplitude was chosen to be $h / c_{0}=0.167$, separated along the span (the 'wavelength') by $\lambda / c_{0}=0.11$. The slit width was chosen to be $w / \lambda=0.12$ and slit dimension ratio $a / w=7.5$, which was shown in Section 6.4.3 to provide optimum noise reductions on a flat plate over 


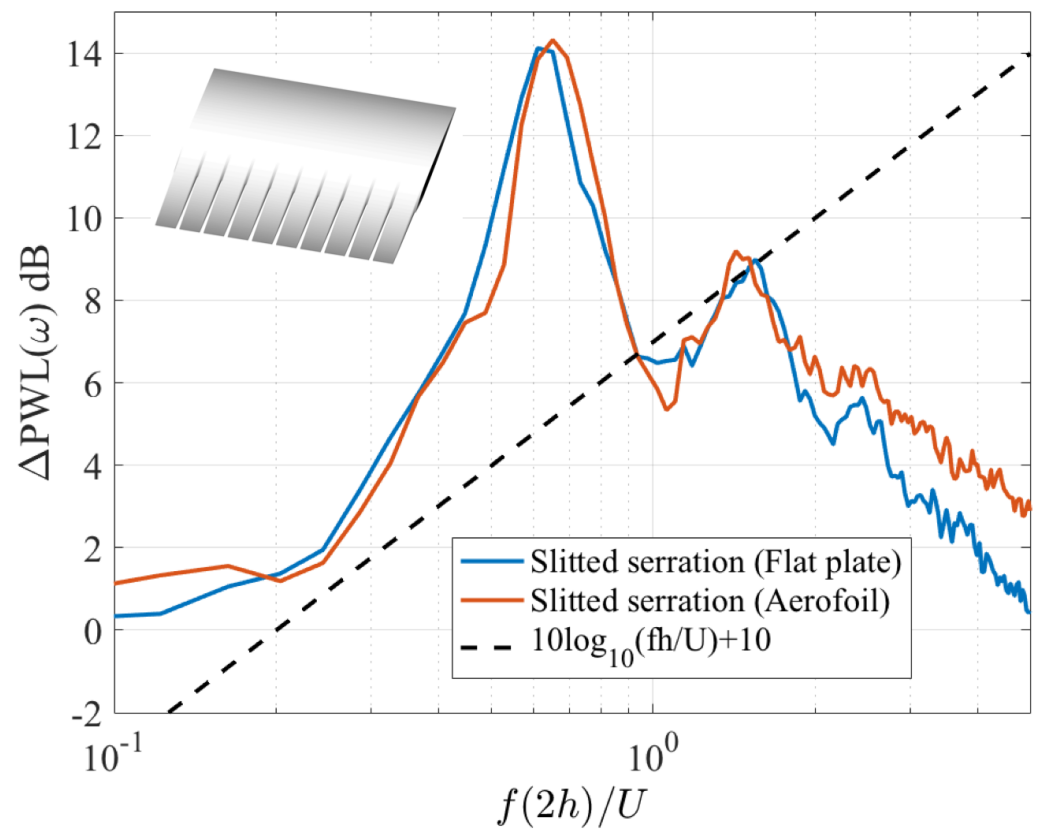

FIGURE 6.19: Sound power reduction comparisons for a slitted serration on flat plate and aerofoil of amplitude $\left(h / c_{0}=0.167\right), w / \lambda=0.133, h_{s} / 2 h=1$ at jet velocity of $60 \mathrm{~m} / \mathrm{s}$.

the frequency bandwidth of interest. A photograph of the slitted profile on the aerofoil leading edge is shown in Figure 6.14.

The sound power reduction spectra for the 3D slitted aerofoil is plotted in Figure 6.19 versus $f(2 h) / U$. Also shown in this figure is the corresponding sound power spectra for the slitted profile on a flat plate.

Levels of noise reduction obtained for the slitted profile on the 3D aerofoil are almost identical to that for the flat plate at frequencies below about $2 f h / U \approx 2.5(3 \mathrm{kHz})$. Above this frequency, 3D flow effects can be seen to influence the noise reductions by approximately $2 \mathrm{~dB}$ compared to a flat plate. Note that no attempt has been made to optimize this $3 \mathrm{D}$ slitted-root geometry. Additional noise reductions may therefore be obtained, particularly at low frequencies (less than $\omega_{0}$ ) by further tuning of the slit width $w$ and wavelength $\lambda$. The flat plate study presented in section 6.4.3 therefore provides a good basis for understanding the physics of leading edge serrations and identifying effective leading edge geometries for reducing interaction noise.

\subsection{V-slit serrations}

The previous sections have demonstrated how very narrow slits introduced into the root of a sawtooth serration can have a significant effect on the level of noise reductions. In this section it is shown that this level of noise of reduction is highly sensitive to the geometry of the slit. In particular, a new slit profile is investigated comprising an inverted triangle ('V - shape') introduced into the serration root, which shall be shown to provide better noise reductions at the tuned frequency but at the expense of reduced noise reductions at lower frequencies. However, one disadvantage of this profile is that effective slit height $h^{*}$ is reduced thus causing an increase in the tuned frequency. 


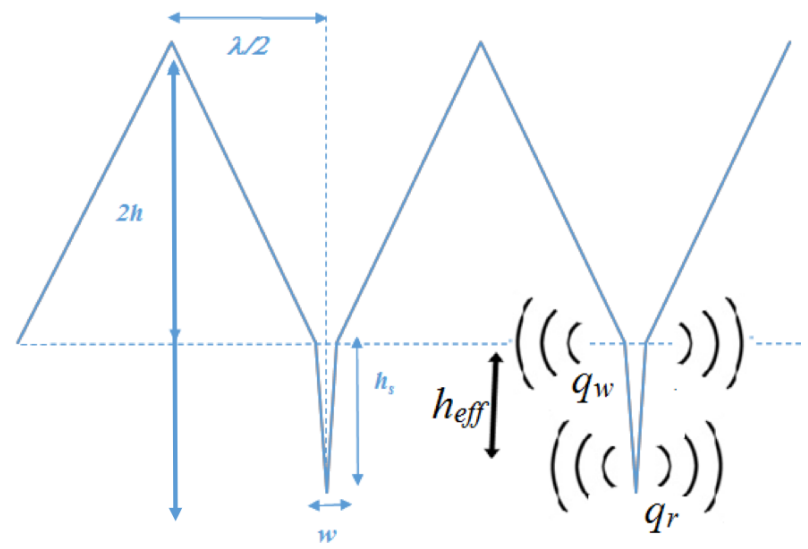

FIGURE 6.20: A Schematic of the leading edge V-slit serrated profile.

Another possible advantage of the V-slit design is that it reduces the cavity volume formed by the slits on 3D aerofoils compared to the rectangular slit cases considered previously. Moreover, the V-slit provides a gradual change in profile whereas it is sudden in the rectangular slit case.

\subsubsection{Geometry}

A Schematic of a 'V-slit' slit geometry is shown below with identical parameters as in the previous section for the rectangular slit. The two dominant noise sources $q_{w}$ and $q_{r}$ are also indicated in the figure 6.20. Here, the effective slit height $h^{*}$ is also indicated which is a key parameter in determining the level of additional noise reductions.

\subsubsection{Acoustic performance on flat plate aerofoils}

The measured sound power reduction spectra due to a V-slit serration, a rectangular slit serration and a non-slitted sawtooth serration are plotted against $f(2 h) / U$ in Figure 6.21a. The length and width of both slits are identical and equal to $h_{s} / c_{0}=0.133$ and $w / \lambda=$ 0.13. The V-slit serration is seen to provide greater maximum noise reductions of almost $15 \mathrm{~dB}$ compared to $14 \mathrm{~dB}$ for the rectangular slit case. The non-dimensional frequency of maximum noise reductions are also observed to be higher, suggesting a smaller effective height $h^{*}$, but smaller levels of noise reduction at lower frequencies.

The noise reduction data plotted in Figure 6.21a is shown again in Figure 6.21b but shown as an additional noise reduction plotted against $\omega / \omega_{0}^{*}$, where $\omega_{0}^{*}$ is the effective tuned frequency defined in terms of the effective slit height $h^{*}, \omega_{0}^{*}=\pi U / h^{*}$. For the case of a rectangular slit of width $w / \lambda=0.13, h^{*}$ becomes $1.12 h_{s}$, whereas for V-slit it is approximately equal to $0.7 h_{s}$. This comparison causes the peaks in the two noise reduction spectra to be aligned at $\omega / \omega_{0}^{*}=1$ thereby allowing the additional noise reduction spectra to be directly compared.

From the previous investigation of the rectangular slit profiles presented in Section 6.3.7, the source strength ratio $r(\omega)$ was found to increase slightly with increasing in slit height (see figure 6.10). Similar observations can be made in the case of the V-slit serration profiles. A consequence of the decrease in effective slit height $h^{*}$ by the V-slit serrations, it is speculated that the source ratio $r(\omega)$ is decreased and hence additional noise reductions are poorer compared to the rectangular slit profile. This findings requires further investigation 

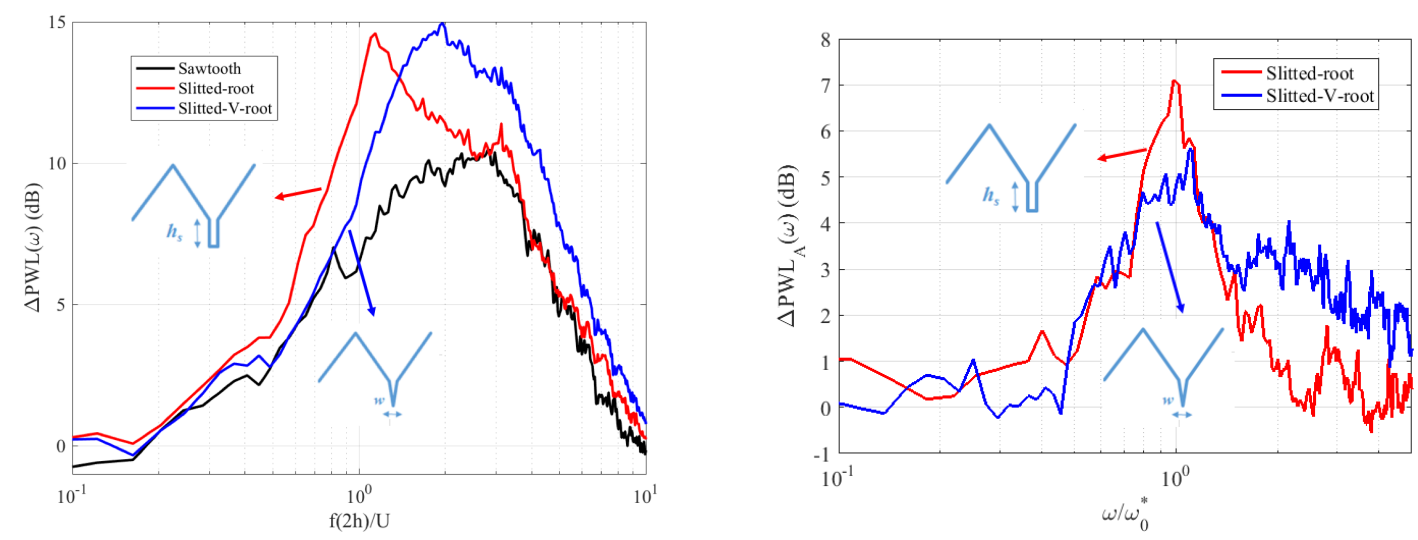

(a) Noise reductions versus non-dimensional frequency (b) Noise reductions versus non-dimensional frequency $\omega / \omega_{0}$. $f(2 h) / U$.

FIGURE 6.21: Sound power reduction comparisons for a V-slit serration of amplitude $\left(h / c_{0}=0.1\right), w / \lambda=0.13, h_{s} / c_{0}=0.133$ with sawtooth at jet velocity of $60 \mathrm{~m} / \mathrm{s}$.

to understand more fully the precise noise reduction characteristics for V-slit serrations, as well as the sensitivity of noise reductions to small changes to the slit geometry. In summary, the geometry of the slit is very important factor in order to match the source strengths to achieve maximum noise reductions.

\subsection{Conclusions}

This chapter extends the noise reduction principles proposed in Chapter 5 to a new profile design that allows the dominant interfering sources to be perfectly aligned in the streamwise direction thereby enhancing their ability to interfere. The new profile simply involves introducing slits onto the roots of sawtooth serration profiles. These new leading edge profiles overcome the fundamental limitation of double-wavelength serrations whose performance were shown to be limited by the imperfect coherence between adjacent roots.

This chapter has presented a leading profile that is capable of providing considerably better reductions in turbulence-aerofoil interaction noise compared to conventional single wavelength or sawtooth serration profiles. The leading edge profile comprises simply of narrow slits introduced on the airfoil leading edge. By the simple addition of slits, a new control mechanism is introduced involving the destructive interference between two compact source regions approximately located at either ends of the slit. Maximum noise reductions are achieved at a frequency, which we referred to in this chapter, as the tuned frequency at which these two sources radiate $180^{\circ}$ out of phase. Greatest noise reductions are therefore achieved when the two source strengths at either ends of the slit are equal at the tuned frequency. This balancing of source strengths is achieved by the careful choice of slit width. Based on this principle, this chapter has demonstrated noise reductions at a flow speed of $40 \mathrm{~m} / \mathrm{s}$ of almost $18 \mathrm{~dB}$ compared with noise reductions of just $7 \mathrm{~dB}$ for a sawtooth un-slitted profile of the same overall amplitude.

This chapter has demonstrated conclusively that single wavelength or sawtooth leading edge profiles, that have been widely investigated in previous work, provide levels of noise reduction that are significantly inferior to those achievable by the new design. Further work 
is needed to understand the factors that control the source strength distribution along the edge to optimize the leading edge profile further. Further refinement of the slitted profiles are also possible that may be rounder and avoid sharp corners as long as the source balance is not affected. Finally, further work is needed to quantify the effect of slits on aerodynamic performance. Any degradation in aerodynamic performance must of course be balanced against the positive effects of reduced noise.

However, a small increase in noise at very high frequencies was observed on 3D aerofoils which is most likely due to the effect of a resonance occurring in the cavity formed by the two parallel sides of the slit. An alternative slitted geometry comprising a ' $\mathrm{V}$ ' shape is also considered to minimise this effect. It has been shown that this slitted-V profile provides better noise reductions at high frequencies. 



\title{
Chapter 7
}

\section{Enhanced noise reductions using variable-slit and chopped-peak leading edge serrations}

\author{
Contents

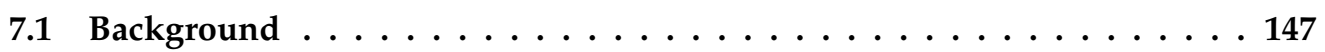 \\ 7.2 Variable-slits serrations . . . . . . . . . . . . . . . . . . . . 149 \\ 7.2 .1 Geometry . . . . . . . . . . . . . . . . . . . . . . . . . 149 \\ 7.2 .2 Control principle . . . . . . . . . . . . . . . . . . . . . . . . . . 149 \\ 7.2.3 Acoustic performance on flat plates . . . . . . . . . . . . . 150 \\ 7.2.4 Influence on noise reductions due to $a$ and $b \ldots \ldots \ldots \ldots$ \\ 7.2.5 Simplified analytical model to predict additional noise reductions . . 152 \\ 7.2.6 Influence on noise reductions due to flow speed . . . . . . . . 155 \\ 7.2.7 Predicted dependence of $\Delta \mathrm{PWL}_{I}$ on $N$ and $\lambda$ on noise reductions . . 155 \\ 7.3 Chopped-peak serrations . . . . . . . . . . . . . . 156 \\ 7.3 .1 Geometry . . . . . . . . . . . . . . . . . 157 \\ 7.3 .2 Control principle . . . . . . . . . . . . . . . . . . . . 157 \\ 7.3.3 Acoustic performance on flat plate aerofoils . . . . . . . . . . 158 \\ 7.3.4 Influence of noise reductions due to flow speed . . . . . . . . 158 \\ 7.3.5 Simple analytical model to predict additional noise reductions . . . . 159 \\ 7.4 Independence of low frequency noise reductions to serration geometry . 160
}

In Chapters 5 and 6, two new leading edge serration profiles were prosed to provide substantially better noise reduction than conventional single frequency sawtooth profiles alone. These new concepts exploited the finding that the dominant noise source on the leading edge were concentrated at the root. In this chapter, two new leading edge designs are proposed aimed at overcoming the limitations of the two previous designs. Their effectiveness is validated experimentally on flat plates and compared against relatively simple analytic models.

\subsection{Background}

In Chapters 5 and 6, it was shown that the double-wavelength and slitted-root serrations provide considerably better noise reductions compared to single-wavelength profiles at the optimum wavelength. However, these profiles may suffer from three limitations: 


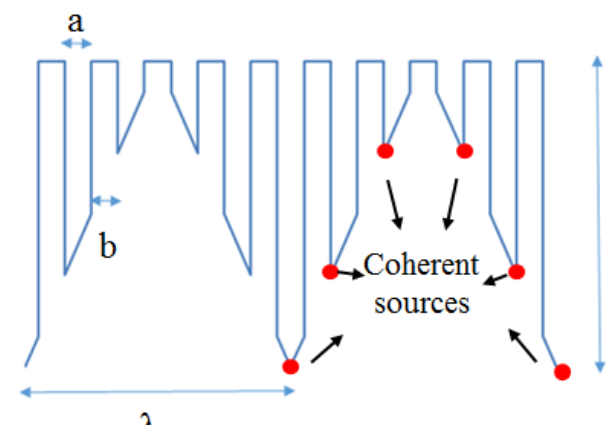

$\lambda$

a) Variable-slit serration

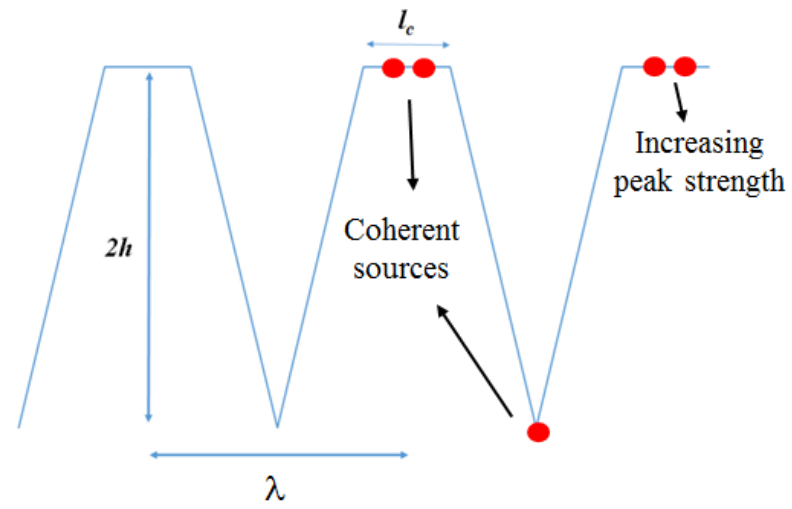

b) Chopped-peak serration

FIGURE 7.1: A sketch of the two leading edge profiles a)Variable-slit serrations, b) Chopped-peak serrations.

1. Noise Control is tuned to a single frequency, which then falls off quite rapidly at frequencies away from this.

2. Structural limitations.

3. Potential loss in lift and drag.

The two new leading edge profiles presented in this chapter are designed to overcome some of these issues. However, these two will also be shown to provide superior noise reductions compared to previous designs for the same peak-to-root distance.

A sketch of the two profiles are shown in figure 7.1, indicating the principal dimensions of each profile and the location of the dominant sources along the leading edge (inferred from the noise reduction spectra).

The profiles proposed are described as follows:

1. Variable-slit leading edge profiles in which multiple, regularly spaced slits are formed of varying lengths along the span. In Figure 7.1, the slit heights are arranged to form a sawtooth pattern although any geometry could be formed. The principle behind this design is simply to provide a series of compact sources at the end of each slit, as shown by the red dots in figure 7.1. Clearly, effective interference is only possible when the root sources are located closer together than the optimum wavelength defined in Chapter 4. The noise control principle involved in this serration profile is therefore a combination of those for the double wavelength serration in Chapter 5 and the slitted root serration in Chapter 6.

2. Chopped-peak leading edge profiles in which the source strength at the peak is deliberately increased by clipping the peak of a conventional sinusoidal or sawtooth serration so that it more effectively interferes with the other dominant sources, leading to improved noise reductions. In addition to achieving better noise reductions than double-wavelength serrations, it may also achieve better aerodynamic performance since most of the span comprises of straight leading edges, as shown in figure 7.1.

Similar to previous chapters, in this chapter the results of a systematic parametric study are presented on flat plates using the procedure described in Appendix A. 


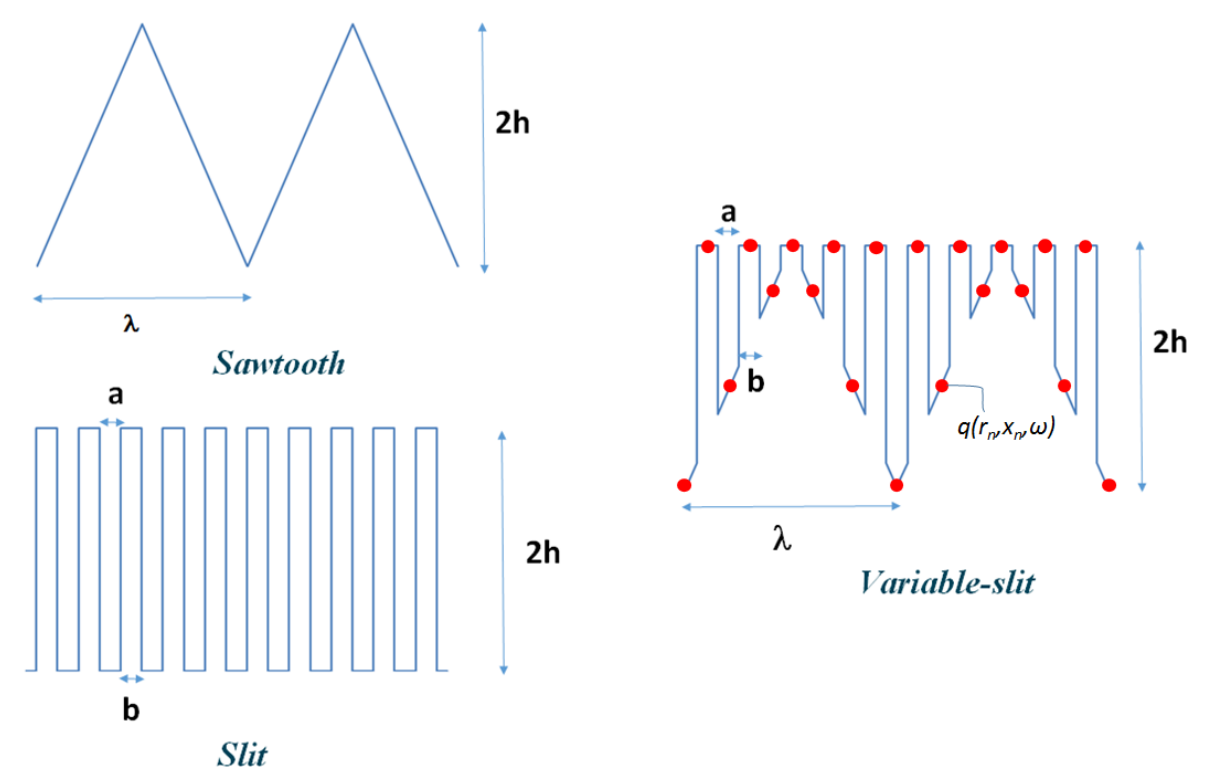

FIGURE 7.2: A Schematic of the leading edge Variable-slits serrations.

\subsection{Variable-slits serrations}

\subsubsection{Geometry}

A schematic of a 'Variable-slit' geometry is shown in figure 7.2 with parameters of serration amplitude $2 h / c_{0}$, serration wavelength $\lambda / c_{0}$, and slit width $a$ and distance between adjacent slits $b$. A variable-slit geometry may be formed by the combination of a sawtooth and a slitted serration profile as shown in figure 7.2. The main goal of this profile is to provide dominant compact sources located at the end of slit that are of different streamwise distances from the leading edge. Red dots in figure 7.2 denote the likely positions of the dominant sources on the variable-slit serration which is later used as the basis for the development of an analytical model to predict the additional noise reductions in section 7.2.5. A total of 8 variable-slit serrations are investigated experimentally to understand the noise reduction characteristics and mechanism.

\subsubsection{Control principle}

The noise control principle underlying the variable-slit serration profile combines the control principles of both the slitted and double-wavelength serrations. In this design, multiple dominant sources are distributed in the streamwise direction such that they interfere. Similar to the double-wavelength serrations, adjacent root sources will be partially coherent due to their distribution along the spanwise direction as shown in figure 7.2. The advantages of this design is that it allows for a distribution of discrete compact root sources along an arbitrary profile and therefore has more degrees of freedom than the two previous designs that provide noise reductions through the interference between just two compact root sources. The variable slit profile also represents a smaller modification to the baseline straight edge profile since it simply consists of a series of very narrow slits of varying lengths.

Since the variable slit profile provides a distribution of compact root sources closer together than the optimum wavelength $\lambda_{0}$ the noise reduction spectrum is more complex than for the 
previous designs. A model to describe the mechanism for the additional noise reductions is discussed in section 7.2.5.

When the slit width $a$ is sufficiently smaller than the distance between adjacent slits $b$, the sources at the root will be dominant and interference will only occur between adjacent root sources. However, as $b$ is increased the source strength at the peak becomes significant compared to the strength at the root, leading to further interference between the peak and root.

In general, overall sound power reductions provided by these variable-slit profiles therefore comprises the sum of three contributions. The first arises from the reduction in source strength along the leading edge, which we shall denote as $\Delta \mathrm{PWL}_{S}$. In this case, the reduction of source strength $\triangle \mathrm{PWL}_{S}$ is the noise reductions due to slitted serrations as shown in figure 7.2. The second arises from interference between adjacent root sources, which we shall denote as $\Delta \mathrm{PWL}_{I r, r}$. The third contribution to the noise reduction arises from interference between root and peak sources, which we shall refer to as $\Delta \mathrm{PWL}_{I r, p}$. The overall sound power reduction may therefore be written as,

$$
\Delta \operatorname{PWL}(\omega)=\Delta \mathrm{PWL}_{S}(\omega)+\Delta \mathrm{PWL}_{I r, r}(\omega)+\Delta \mathrm{PWL}_{I r, p}(\omega) .
$$

A simple analytical model to predict the additional noise reduction is developed in Section 7.2.5 below based on this hypothesis. The experimental data confirming the proposed noise reduction mechanism and effectiveness of the of variable-slit serrations is now presented. Without knowing the variation of relative source strength of peak and root with frequency, it is difficult to separate $\mathrm{PWL}_{I r, r}$ and $\Delta \mathrm{PWL}_{I r, p}$ from the measured data. Thus in this chapter general terminology $\Delta \mathrm{PWL}_{I}$ is used to represent $\Delta \mathrm{PWL}_{I r, r}+\Delta \mathrm{PWL}_{I r, p}$ for measured data.

\subsubsection{Acoustic performance on flat plates}

Figure 7.3 shows the comparison of the noise reduction spectra between a variable-slit serration profile, a slitted profile and a sawtooth profile plotted against non-dimensional frequency $f(2 h) / U$ for the same relatively short serration amplitude of $h / c_{0}=0.1$ and wavelength $\lambda / c_{0}=0.2$ and flow velocity of $60 \mathrm{~m} / \mathrm{s}$. In this present study the slit dimensions considered are $a / \lambda 0.066, b / \lambda=0.133$. The variable-slit serration can be seen to provide greater noise reductions at lower non-dimensional frequencies (especially below $f(2 h) / U<1$ ) than those of the sawtooth and slitted serrations. A noise increase in high frequencies is observed both in slitted and variable-slit serration designs. The reason for this behavior might be due to cross flow across the slit. Finally, for the same peak-to-root distance, variable-slit serrations provide substantially greater noise reductions at low frequencies than those of conventional non-slitted profiles. A more details about the noise reductions characteristics for slitted serrations are presented in Appendix D.

\subsubsection{Influence on noise reductions due to $a$ and $b$}

To make explicit that overall noise reductions due the variable-slit serrations arises from interference of compact sources located on the slit roots for cases in which the root sources are dominant, a systematic parametric study has been performed by varying slit widths $a$ and $b$ whilst keeping the number of slits per wavelength constant at $(a+b) / \lambda=0.2$. 


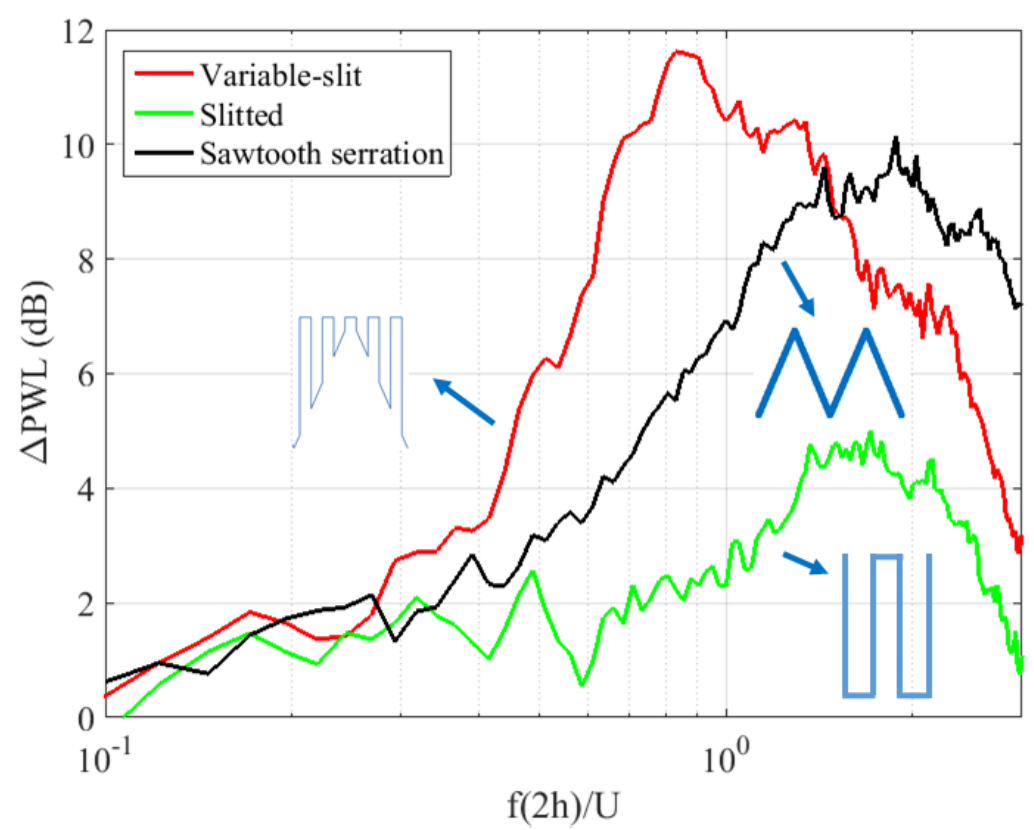

FIGURE 7.3: Acoustic performance of Variable-slits serrations for serration amplitude $\left(h / c_{0}=0.1\right)$, serration wavelength $\left(\lambda / c_{0}=0.2\right)$ at jet velocity of 60 $\mathrm{m} / \mathrm{s}$.

Figure 7.4a shows the noise reduction spectra versus non-dimensional frequency $f(2 h) / U$ for 3 different slit widths $a / \lambda$ of $0.133,0.1,0.066$ at a fixed serration amplitude $h / c_{0}=0.1$, serration wavelength $\lambda / c_{0}=0.2$, flow speed of $60 \mathrm{~m} / \mathrm{s}$ for $(a+b) / \lambda=0.2$. Also shown in this figure is the line $10 \log _{10}(f h / U)+10$ corresponding to the maximum sound power reduction versus frequency that can be obtained by a conventional sawtooth serration.

Figure 7.4a shows that the noise reductions are highly sensitive to slit dimensions $a$ and $b$. As the slit width $a / \lambda$ is varied, the largest variation in noise reductions occur at the frequency $f(2 h) / U=0.81$. However, the precise relationship between the level of noise reduction and variations in the slitted serration parameters is highly complex since it involves the interaction between a large number of sources, their mutual and relative source strengths. Reducing slit width $a / \lambda$ can be seen to have two effects on the noise reduction mechanism. The first is that reduced root sources strengths are desirable for low noise. The second is that reducing the root source strength causes it to become more comparable to that at the peak thereby causing them to more effectively interfere, leading to enhanced levels of noise reduction.

To illustrate more clearly the effect of the interaction between the various sources, noise reductions due to the variable slit serrations plotted in Figure 7.4a is repeated but for larger overall amplitude $h / c_{0}=0.167$ (i.e, $70 \%$ longer). The noise reduction for these longer serration are plotted in figure 7.4b. Comparing the noise reduction spectra in Figures 7.4a and b demonstrates that the longer serrations provide greater levels of noise reduction compared to the shorter case but, more significantly, an additional noise reduction peak is observed at $f(2 h) / U=2.4$ (indicated in the figure 7.4b), which we shall later show is due to interference between the peak and root. This additional peak cannot be observed in the case of the shorted serrations since it is masked by self noise.

Note that the additional noise reductions are not plotted because the reduction in source strength $\Delta \mathrm{PWL}_{S}$ is not constant for the different slit width cases. 


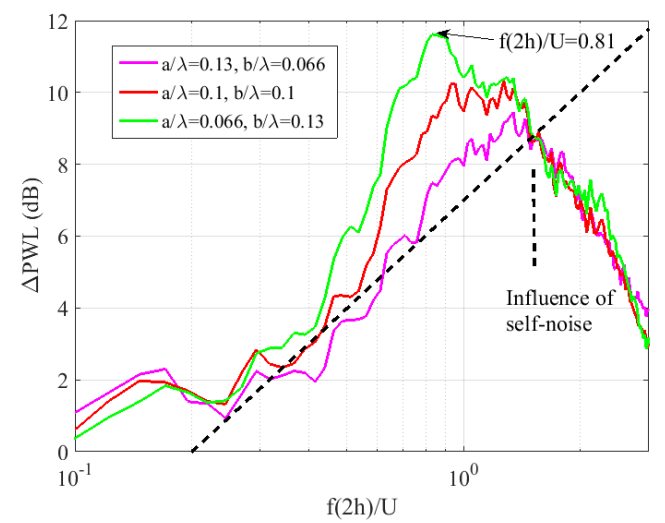

(a) Influence of slit width for $h / c_{0}=0.1, \lambda / c_{0}=0.2$.

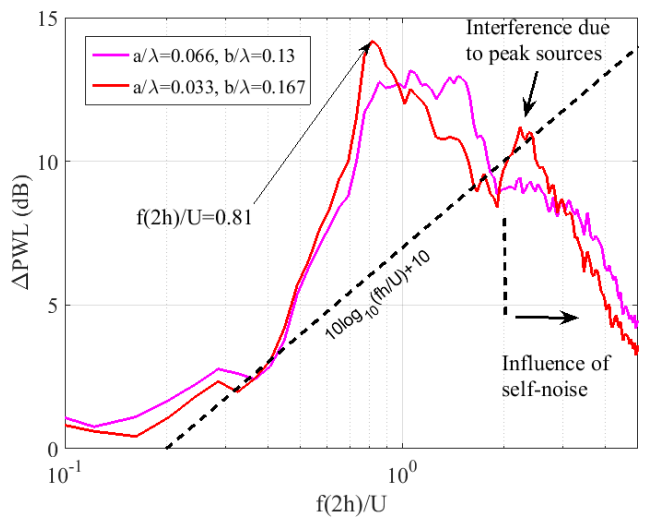

(b) Influence of slit width for $h / c_{0}=0.167, \lambda / c_{0}=0.2$.

FIGURE 7.4: Acoustic performance of variable slit serration of same amplitude at jet velocity of $60 \mathrm{~m} / \mathrm{s}$.

\subsubsection{Simplified analytical model to predict additional noise reductions}

In this section a simple analytical model is presented to understand the behaviour of the noise reductions with serration geometry observed experimentally above. The model will focus on the additional noise reduction $\Delta \mathrm{PWL}_{I}$ due to interference between adjacent root sources.

In this simple model we only consider the case where root sources are dominant. At the present time it is unclear what the combination of geometric parameters and frequencies lead to this condition but it is certainly related to having sufficiently large slit width $a$, which the noise reduction data in Chapter 6 suggests should be greater than approximately $1 \mathrm{~mm}$. In the model it is assumed that the root sources have identical source strength but radiate to a far-field observer with a phase difference $\omega l / U$ arising from the streamwise separation distance $l$ between two root sources. Consider $N$ compact root sources located at every root positions $r_{n}$ and streamwise positions $x_{n}$ with rms source strengths $q\left(r_{n}, x_{n}, \omega\right)$ along the airfoil span, as shown in figure 7.2. The path length differences between adjacent roots to a far-field observer can be neglected since they are generally much closer together compared to an acoustic wavelength. The total radiated pressure at any given frequency $\omega$ is therefore proportional to the sum of the source strengths $q\left(r_{n}, x_{n}, \omega\right)$ with relative phase difference relative the source $n=1$ included,

$$
p(\omega) \propto \sum_{n=1}^{N} q\left(r_{n}, x_{n}, \omega\right) e^{-i \omega\left(x_{n}-x_{1}\right) / U}
$$

The radiated sound power $W_{s}$ is related to the mean square far-field pressure integrated over some suitable closed surface. As discussed earlier in section 4.4.4, the mean square pressure will depend on the degree of coherence between adjacent sources on the serrated leading edge, which is related to the serration wavelength compared to the turbulence integral length scale. Following Eq. 7.2, the far-field mean square pressure, and hence radiated sound power, can therefore be written as,

$$
W_{s}(\omega) \propto \overline{p^{2}}(\omega) \propto \mathbf{E}\left[p^{*}(\omega) p(\omega)\right]
$$


Substituting equation 7.2 in equation 7.3, the radiated sound power becomes,

$$
W_{s}(\omega) \propto \sum_{n=1}^{N} \sum_{n^{\prime}=1}^{N} \mathbf{E}\left[q^{*}\left(r_{n}, x_{n}, \omega\right) q\left(r_{n^{\prime}}, x_{n^{\prime}}, \omega\right)\right] e^{-i \omega\left(x_{n^{\prime}}-x_{n}\right) / U},
$$

Following an identical argument to the analyses presented in sections 5.6 and 4.4.4 for homogeneous turbulence, the source strength cross spectra in Eq 4.4 can be written in terms of the coherence function as,

$$
\mathbf{E}\left[q^{*}\left(r_{n}, x_{n}, \omega\right) q\left(r_{n^{\prime}}, x_{n^{\prime}}, \omega\right)\right]=\overline{q^{2}}(\omega) \gamma^{2}\left(\left|r_{n}-r_{n^{\prime}}\right|, \Lambda_{t}, \omega\right)
$$

where $\overline{q^{2}}(\omega)$ is the mean square source strength at the root location. As in previous analyses a simple exponential form for the source strength spanwise coherence function is assumed defined by Eq. 5.7,

$$
\gamma^{2}\left(\left|r_{n}-r_{n^{\prime}}\right|, \Lambda_{t}, \omega\right)=e^{-\left|r_{n}-r_{n^{\prime}}\right| / l(\omega)}
$$

where $l(\omega)$ is the frequency-dependent coherence length as given in equation 5.13.

Substituting equation 7.5 in equation 7.4 and by using equation 7.6 , the radiated sound power for variable-slit serrations becomes,

$$
W_{s}(\omega) \propto \overline{q^{2}}(\omega) \sum_{n=1}^{N} \sum_{n^{\prime}=1}^{N} e^{-\left|r_{n}-r_{n^{\prime}}\right| / l(\omega)} e^{-i \omega\left(x_{n^{\prime}}-x_{n}\right) / U},
$$

Equation 7.7 for the sound power radiated by the variable-slit serration will now be compared to the sound power $W(\omega)$ of a baseline case corresponding to a single slit of the same amplitude and the same value of $a+b$ since the root source strengths in both cases is the same. This can be calculated within the present theoretical framework by assuming zero phase difference, i.e., substituting $x_{n^{\prime}}-x_{n}=0$ in (7.7), to give,

$$
W(\omega) \propto \overline{q^{2}}(\omega) \sum_{n=1}^{N} \sum_{n^{\prime}=1}^{N} e^{-\left|r_{n}-r_{n^{\prime}}\right| / l(\omega)} .
$$

The additional sound power reduction due to interference between the $N$ root sources relative to the single slit case may therefore be written as $\Delta \mathrm{PWL}_{I r, r}(\omega)=W(\omega) / W_{s}(\omega)$ (defined to be positive), which may be further expressed as a double summation over all possible source combinations,

$$
\Delta \mathrm{PWL}_{I r, r}(\omega)=10 \log _{10}\left[\frac{\sum_{n=1}^{N} \sum_{n^{\prime}=1}^{N} e^{-\left|r_{n}-r_{n^{\prime}}\right| / l(\omega)}}{\sum_{n=1}^{N} \sum_{n^{\prime}=1}^{N} e^{-\left|r_{n}-r_{n^{\prime}}\right| / l(\omega)} e^{-i \omega\left(x_{n^{\prime}}-x_{n}\right) / U}}\right] .
$$

As shown for the case of the double-wavelength serration in section 5.6, the coherence between two spanwise locations is only a function of $(a+b) / \Lambda$ and non-dimensional frequency $\omega / \omega_{\Lambda}$.

To assess the validity of the noise reduction mechanism described by Eq. 7.9 for predicting the noise reductions additional to the baseline (slitted geometry), predictions from this 


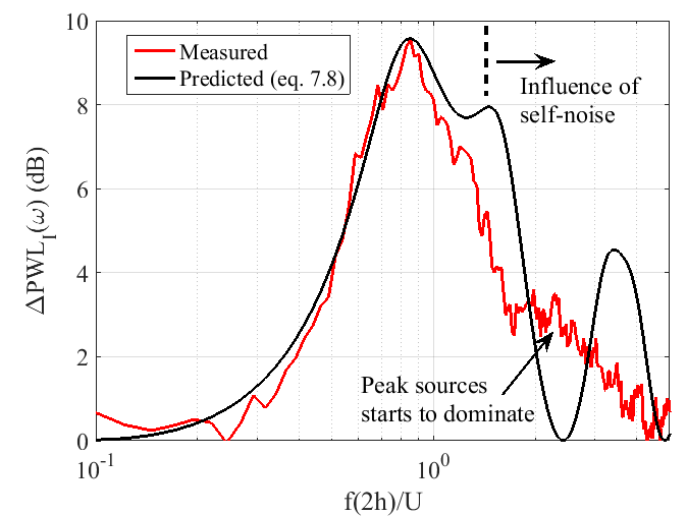

(a) $h / c_{0}=0.1$.

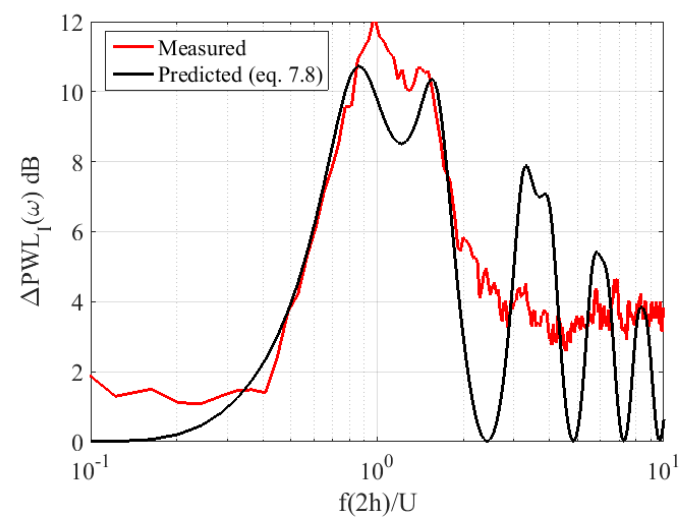

(b) $h / c_{0}=0.167$.

FIGURE 7.5: Comparison between analytical and experimental additional noise reductions for slitted-root serrations of $\lambda / c_{0}=0.2$ at jet velocity $\mathrm{U}=60$ $\mathrm{m} / \mathrm{s}$.

equation are compared to the measured additional noise reduction spectra for the two cases of peak-to-root distance $h / c_{0}$ of $0.1,0.167$, slit widths $a / \lambda=0.066, b / \lambda=0.13$ and flow speed of $60 \mathrm{~m} / \mathrm{s}$. This geometry corresponds to 6 slits per wavelengths and therefore 6 root sources with streamwise and spanwise locations of $x_{n} / 2 h=0,0.41,0.82,0.82,0.41,0$ and $r_{n} / \lambda=0,0.2,0.4,0.6,0.8,1$ respectively $((a+b) / \lambda=0.2)$. Equation 5.13 in Chapter 5 is used to calculate the coherence length $l(\omega)$ from knowledge of the turbulence integral length scale.

Figure 7.5 shows a comparison between the measured and predicted additional noise reduction spectra for the two different serration heights $h / c_{0}$ of 0.1 and 0.167 at a flow velocity of $60 \mathrm{~m} / \mathrm{s}$. Good qualitative agreement between measurement and prediction is observed, providing confirmation of the validity of the additional noise reduction mechanism described by the model. Most peaks, including those relatively minor peaks, observed in the measured noise reduction spectra are predicted using Eq. 7.9.

One failing of the model, which only includes root sources, is the absence of a peak at $f(2 h) / U$ of 2.4 observed in the measured spectra. The model can be seen to predict a dip in the spectra where a peak has been measured. This additional peak is more clearly seen in the noise reduction spectra in Figure 7.4b corresponding to the largest value of slit width $b$. The reason for this discrepancy is the occurrence of additional interference between the root and the peak source that is not included in the analytic model.

To confirm this assumption Equation 7.9 for the additional noise reductions was computed but now allowing for sources to be located at the peaks of the slit and assuming identical source strengths to those of the root. Clearly, in practice this is not the case but is a useful assumption for estimating the peak frequencies.

Figure 7.6 compares the predicted additional noise reduction spectra for two cases where only root sources are included and again when both root and peak sources are included. As observed in the measured noise reduction spectra in figures $7.4 \mathrm{~b}$ and $7.5 \mathrm{a}$, an additional peak in the noise reduction spectra is predicted at non-dimensional frequency $f(2 h) / U$ of 2.4 , consistent with the measured data. Thus, this provides strong evidence for the existence of the presence of both root and peak sources in the case of variable-slit serrations. 


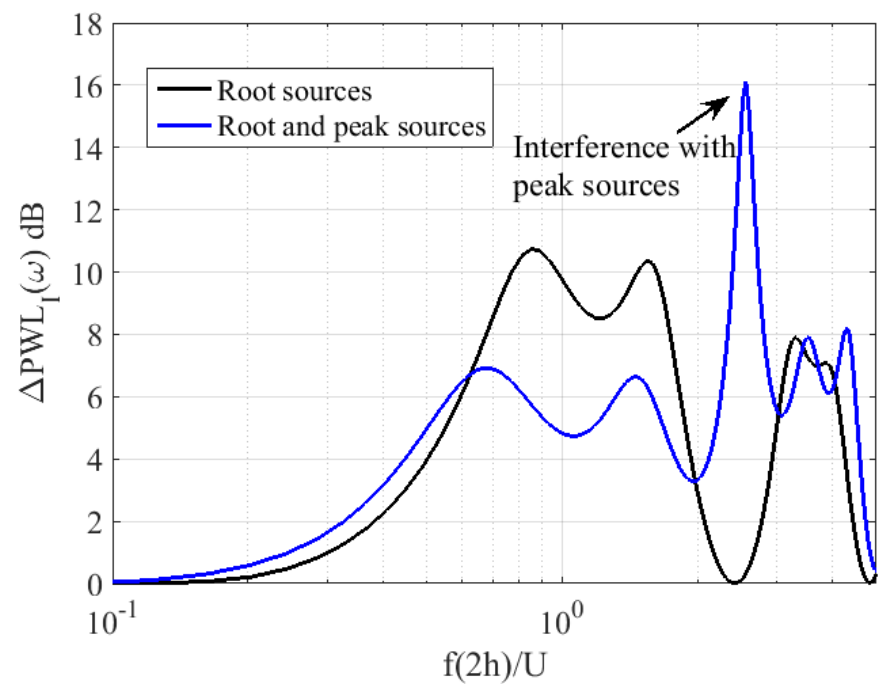

FIGURE 7.6: Significance of peak sources for slitted-root serrations of $h / c_{0}=$ $0.167, \lambda / c_{0}=0.2$ at jet velocity $\mathrm{U}=60 \mathrm{~m} / \mathrm{s}$.

\subsubsection{Influence on noise reductions due to flow speed}

In this section, we investigate the the velocity-dependence of slitted-root serrations on the noise reductions. Figure 7.7 shows the sound power reduction spectra plotted against $f(2 h) / U$ for the case with serration amplitude $h / c_{0}=0.167$, slit dimension $a / \lambda=0.033, b / \lambda=0.167$, and serration wavelength $\lambda / c_{0}=0.2$ at the four flow speeds of $20,40,60$ and $80 \mathrm{~m} / \mathrm{s}$. Collapse of the noise reduction spectra for the variable slit geometry can be seen to be significantly worse than for the previous profiles investigated in this thesis, when plotted against non-dimensional frequency $f L / U$ where $L$ can be any length associated with the geometry. Clearly, therefore, noise reductions are not self-similar, suggesting a different interaction mechanism with the incident turbulent flow. One possibility, that requires further investigation, is that the sources arising from the interaction of turbulent flow with very narrow slits mostly likely involves viscous effects. The noise reduction effectiveness is therefore likely to be Reynolds number dependent but more work is needed to establish this more clearly.

\subsubsection{Predicted dependence of $\Delta \mathrm{PWL}_{I}$ on $N$ and $\lambda$ on noise reductions}

The model described above for predicting the additional noise reduction can be expressed in terms of the three non-dimensional parameters of ratio of spanwise root separation distance relative to the turbulence length scale $\left(r_{n}-r_{n^{\prime}}\right) / \Lambda_{t}\left(l(\omega) \propto \Lambda_{t}\right)$ and two non-dimensional frequencies $\omega / \omega_{\Lambda}$ and $\omega\left(x_{n}-x_{n^{\prime}}\right) / U$.

In this section we interrogate the model developed above to determine the sensitivity of the level of additional noise reductions to the number of root sources per wavelength and the ratio of wavelength $\lambda$ to turbulence length scale $\Lambda_{t}$.

Consider $N$ dominant root sources along the serration profile. For reasons that will be clear below, a sinusoidal profile is assumed in this study. The predicted additional noise reductions are calculated using Eq. 7.9 for three different values of $N=6,20$ and 50 and the results plotted against $f(2 h) / U$ in Figure 7.8a. Also shown in this figure is the line $10 \log _{10}(f h / U)+10$ corresponding to the maximum sound power reduction versus frequency that can be obtained by a sinusoidal serration (cf Chapter 4). 


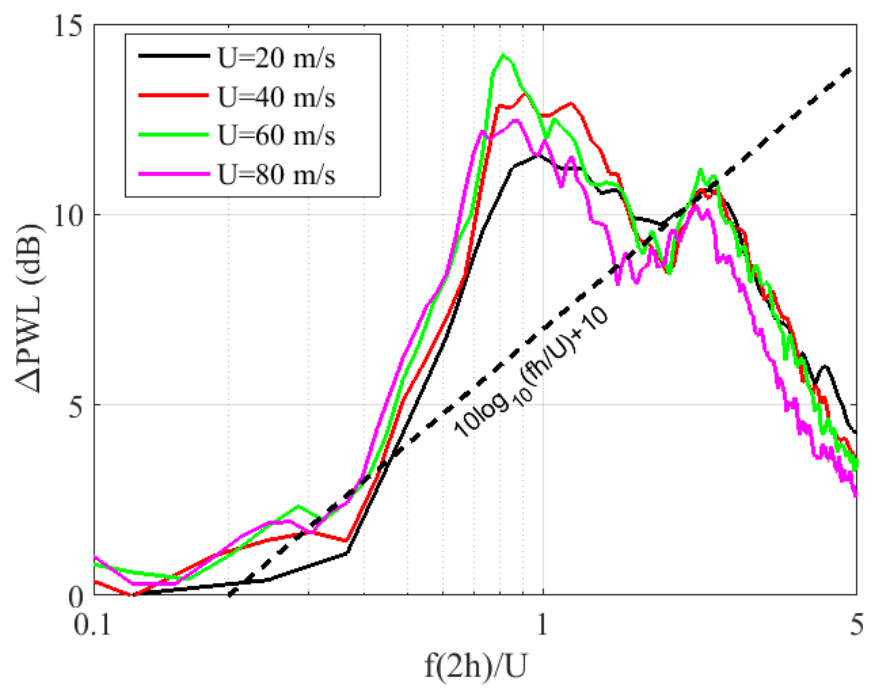

FIGURE 7.7: Influence of jet velocity on noise reduction of variable-slit serrations of slit dimension $a / \lambda=0.033, b / \lambda=0.167$, serration amplitude $h / c_{0}=0.167$ and serration wavelength $\lambda / c_{0}=0.2$.

Additional levels of noise reduction are seen to improve as the number of root source increases, eventually reaching an asymptote for $N=50$. The interesting feature of this results is that the asymptotic noise reduction spectra is very close to the maximum sound power level reduction spectra $10 \log _{10}(f h / U)+10$ that can be achieved by the optimum single wavelength serration profile. This result provides considerable insight into the exact noise reduction mechanism for single wavelength serration considered in Chapter 4 . However, more work is needed to understand this result more fully.

Finally, figure $7.8 \mathrm{~b}$ shows the additional noise reduction spectra versus $f(2 h) / U$ for the 5 different serration-wavelengths $\lambda / \Lambda_{t}$ of 40,20,8,4,2 respectively. Also shown in this figure for comparison is the line $10 \log _{10}(f h / U)+10$. The additional noise reduction spectra in figure $7.5 \mathrm{~b}$ is seen to tend towards $10 \log _{10}(f h / U)+10$ for the two cases less than the optimum wavelength $\lambda / \Lambda_{t}=4$. Again, further work is needed to understand the significance of this result, particularly in relation to the single wavelength serration.

\subsection{Chopped-peak serrations}

This section investigates the noise reduction effectiveness of another type of leading edge profile is investigated that aims to exploit the effects of interference between different dominant sources over the leading edge.

The new profile investigated here provides similar levels of noise reduction as doublewavelength profiles whilst keeping up to $50 \%$ of the span remains as as straight leading edge. This is desirable to minimise the effects of increased drag and a loss in structural integrity. The profile investigated here comprises a conventional sinusoidal or sawtooth profile but whose peak has been 'clipped', as shown in Figure 7.9. The purpose of the clipped peak is to enhance the source strength at the peak to a level comparable to that of the root thereby increasing the effectiveness of interference between the two sources. 


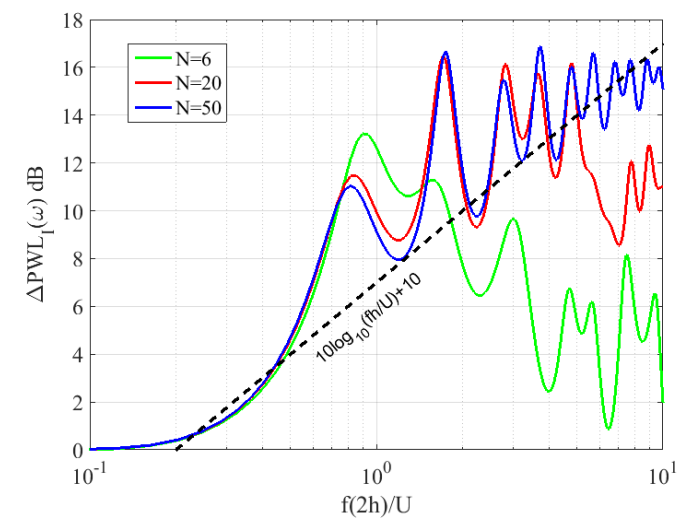

(a) Sound power reduction variation with number of sources $N$.

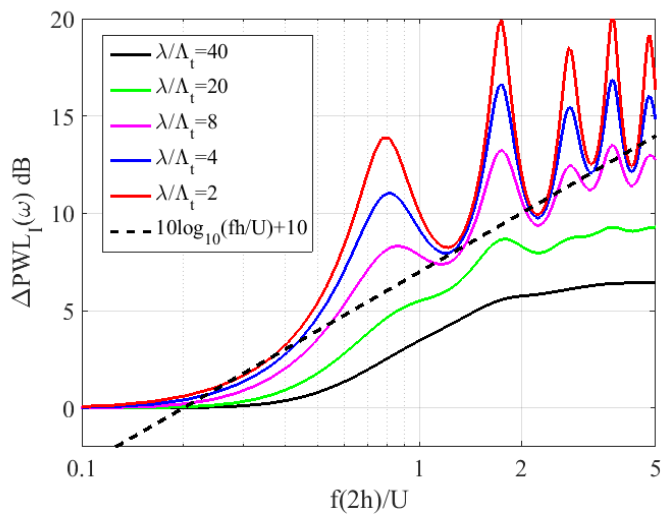

(b) Sound power reduction variation with serration wavelength $\lambda$.

FIGURE 7.8: Predicted behaviour of $\Delta \mathrm{PWL}_{I}$ on $N$ and $\lambda$ to validate the singlewavelength noise reduction hypothesis.

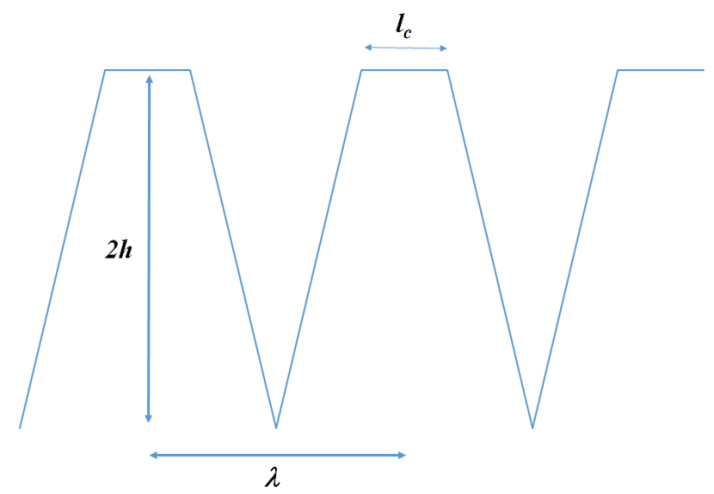

FIGURE 7.9: A Schematic of the leading edge chopped-peak serrated aerofoil.

\subsubsection{Geometry}

Figure 7.9 shows a schematic representation of the chopped-peak design applied to a sawtooth profile, where $l_{c}$ is the width of the clipped peak and $h$ is now the streamwise distance between the root and clipped peak.

The effect on additional noise reductions due to variations in $l_{c}$ are investigated experimentally. In this study, the peak to root distance $2 h$ is maintained constant by modifying the serration inclination angle. The sound power reduction spectra are now compared to the sawtooth baseline configuration whose wavelength is close to the optimum value as discussed in chapter 4 .

\subsubsection{Control principle}

The important difference between the noise reduction mechanism for the chopped-peak design and the previous profiles discussed in this thesis is that interference occurs between sources distributed over the entire leading edge. By contrast, in the previous designs, 


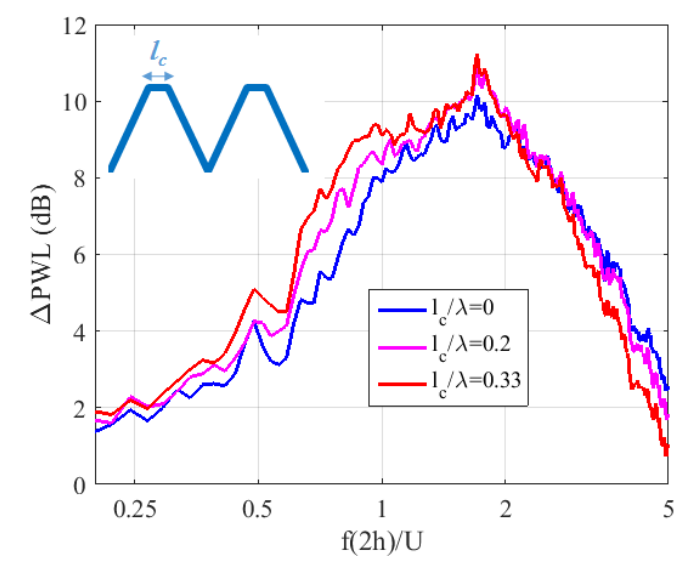

(a) Noise reductions at varied in chopped-peak width $\left(l_{c} / \lambda\right)$.

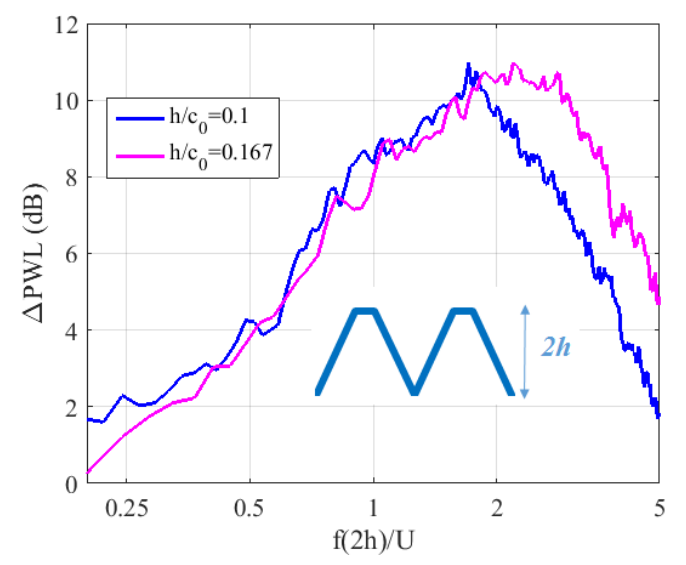

(b) Variation in total serrated amplitude $\left(h / c_{0}\right)$ at a fixed chopped-peak width $l_{c} / \lambda=0.2$.

FIGURE 7.10: Acoustic performance of chopped-peak serrated aerofoils.

interference occurs between a discrete number of compact sources. Owing to the complexity of the noise reduction mechanism, therefore, the additional noise reduction spectra $\left(\Delta \mathrm{PWL}_{I}(\omega)\right)$ will be a complex function of the serration height, chopped-width and the coherence between the peak and root sources.

\subsubsection{Acoustic performance on flat plate aerofoils}

A parametric study was undertaken to investigate the effect on noise reductions due to variations in the width of the chopped peak and the peak to root height. Figure 7.10a shows the variation in noise reduction spectra versus non-dimensional frequency $f(2 h) / U$ at a fixed serration wavelength $\left(\lambda / c_{0}=0.1\right)$ and amplitude $\left(h / c_{0}\right)=0.1$ for the three choppedpeak widths $\left(l_{c} / \lambda\right)$ of $0.1,0.2,0.33$ at a flow velocity of $60 \mathrm{~m} / \mathrm{s}$.

As the width of the chopped-peak $l_{c} / \lambda$ is increased, the figure shows an increase in the low frequency $(f(2 h) / U<1)$ noise reductions. This effect can be attributed to an increase in the source strength at the peak, thereby enhancing the degree of interference between the peak and rest of the leading edge sources.

Finally, the similarity property of the noise reduction spectra is demonstrated even for this relatively complex geometry. Figure $7.10 \mathrm{~b}$ shows the noise reduction spectra at a flow velocity of $60 \mathrm{~m} / \mathrm{s}$ for two different heights $h / c_{0}$ of 0.1 and 0.167 but scaling all dimensions by a factor 1.67 thereby preserving the ratios of all the dimensions. Good collapse of the noise reduction spectra are obtained. As before, deviation at high frequencies is due to the presence of self noise.

\subsubsection{Influence of noise reductions due to flow speed}

Finally, in this section the effect of velocity on the noise reductions due to chopped-peak serrations is investigated. Figure 7.11 shows the sound power reduction spectra plotted against $f(2 h) / U$ for the case with a chopped-peak width of $l_{c} / \lambda=0.33$, serration amplitude $h / c_{0}=0.167$ and serration wavelength $\lambda / c_{0}=0.1$ at the four flow speeds of 20, 40, 60 and 


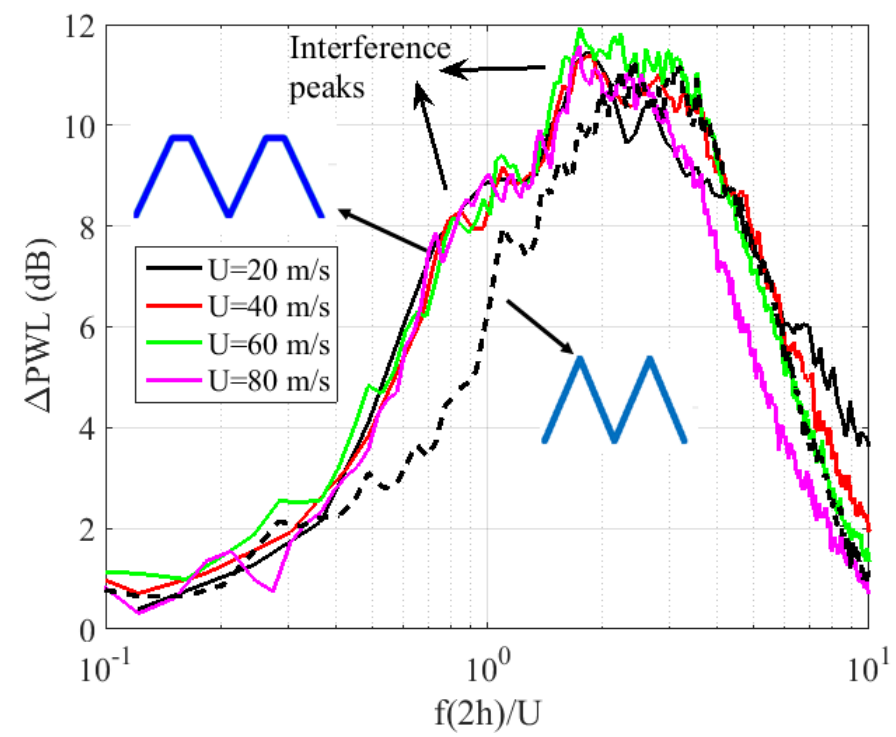

FIGURE 7.11: Influence of jet velocity on noise reduction of chopped-peak serrations of chopped-peak width $l_{c} / \lambda=0.33$, serration amplitude $h / c_{0}=$ 0.167 and serration wavelength $\lambda / c_{0}=0.1$.

$80 \mathrm{~m} / \mathrm{s}$. Similar to the previous serration profiles, almost perfect collapse of the noise reduction spectra are obtained when plotted against non-dimensional frequency $f(2 h) / U$. Noise reductions due to sawtooth serrations are also plotted in the same figure to demonstrate the advantage of chopped-peak serrations across different flow speeds. Two additional noise reduction peaks are observed in the spectra indicated on the figure. A simple model is proposed below to explain this additional noise reduction peak due to chopped-peak serrations.

\subsubsection{Simple analytical model to predict additional noise reductions}

To predict the additional noise reduction for the chopped peak serrations, the model developed in section 7.2.5 for the variable slit serration is used. The purpose of applying this model to the current profile, which includes the effect of a distribution of discrete compact sources along the leading edge, is simply to explain the reason for the existence of the additional noise reduction peaks indicated by the arrows in figure 7.11. Note that this model is not used to determine the level of noise reductions since the precise distribution of source strengths is unknown.

Consider a chopped-peak profile whose coordinates is given by the function $x_{n}=g\left(r_{n}\right)$ whose maximum peak-to-root distance is $2 h$. A chopped-peak serration of reasonably large amplitude $2 h / c_{0}$ of 0.33 , chopped-peak width $\left(l_{c} / \lambda\right)=0.3$, and a wavelength $\lambda / c_{0}$ of 0.1 is considered. Eq. 7.9 is used to predict the noise reduction spectra based on atotal of $N=50$ discrete sources per wavelength of constant source strength. The results for this prediction are plotted in Figure 7.12 against $f(2 h) / U$ and compared against the measured noise reduction spectra. Note that the predictions are based on considering more sources on chopped peak than inclined edge. This is simply to show agreement between the peak frequencies and not absolute levels. Good agreement is observed between the predicted frequencies of maximum noise reduction and the measured values of $f(2 h) / U=0.81,1.6,2.5$, thereby confirming the validity of this simple model for explaining the role of interference between all edge sources in determining the peak frequencies. 


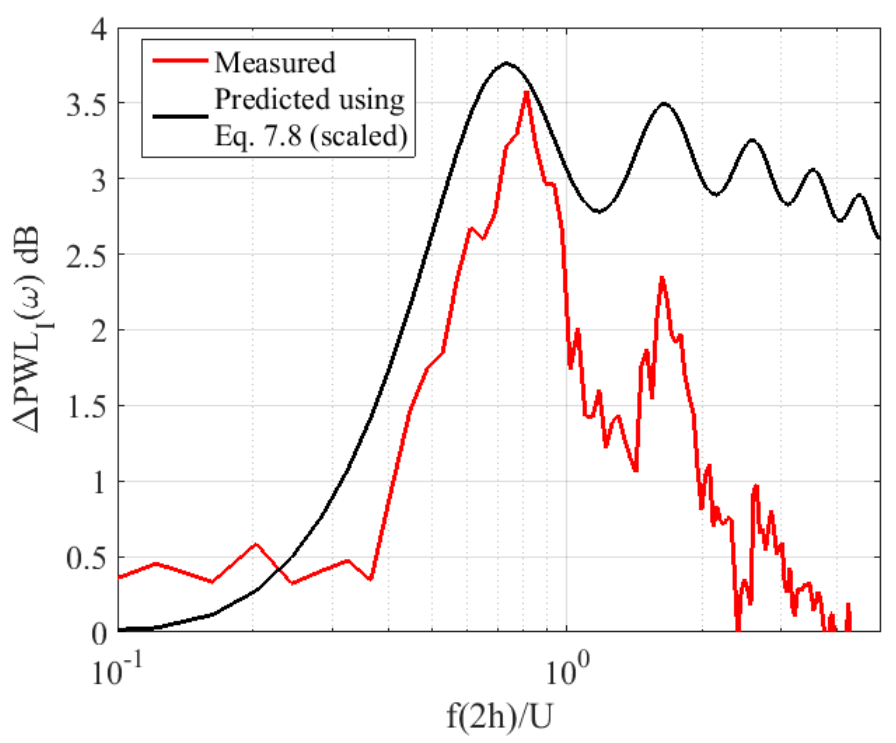

FIGURE 7.12: Comparison between analytical and experimental additional noise reductions for Chopped-peak serrations for $l_{c} / \lambda=0.33,2 h / c_{0}=0.33$, $\lambda / c_{0}=0.1 \mathrm{U}=60 \mathrm{~m} / \mathrm{s}$.

\subsection{Independence of low frequency noise reductions to serration geometry}

Finally, this chapter concludes with a brief study aimed at demonstrating the hypothesis that the relative source strengths along the leading edge is the governing factor in determining the noise reduction due to source effects, $\Delta P W L_{S}$. An important consequence of this hypothesis is that, at sufficiently low frequencies, noise reductions are independent of serration profile, as shall be demonstrated below.

Figure 7.13a is a schematic of the chopped-root design where the root has been truncated. The purpose of this design is to cause a substantial increase in the source strength at the root and assess its effect on the noise reduction spectra. Figure $7.13 \mathrm{~b}$ shows the noise reduction spectra for the chopped-root serration compared against sawtooth serration. Also shown is the noise reduction spectra due to a combined chopped-peak and chopped-root serration.

The important findings of this spectral comparison in Figure 7.13b is that the noise reduction spectra at frequencies below $f(2 h) / U=1$ are almost identical. However, significant differences can be observed at higher frequencies. This result is consistent with the difference in noise reduction spectra between the sawtooth and sinusoidal serration profiles, shown in Figure 6.1 in Chapter 6.

This is one of the most significant conclusions of this thesis. It shows that, in the low frequency range $f(2 h) / U<1$, noise reductions are solely determined by the integrated phase difference along one serration wavelength, which of course is only determined by the peakto-root height $h$ in relation to the hydrodynamic wavelength $U / f$ and hence noise reductions collapse on $f h / U$. The precise geometry, therefore, is unimportant to the level of noise reductions in this low frequency range. Further evidence to support this conclusion is presented in Appendix D showing the noise reductions due to leading profile generated by a Gaussian random distribution with standard deviation $\sigma$. Noise reduction are shown to collapse on $f \sigma / U$. 


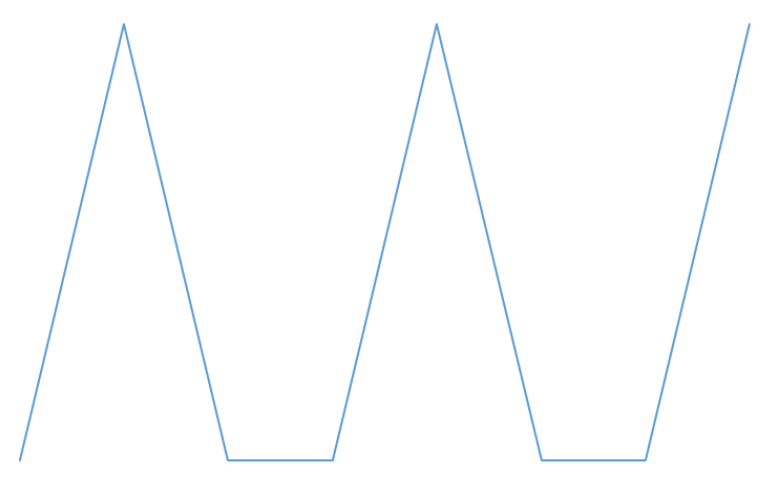

(a) Schematic of the chopped-root serration

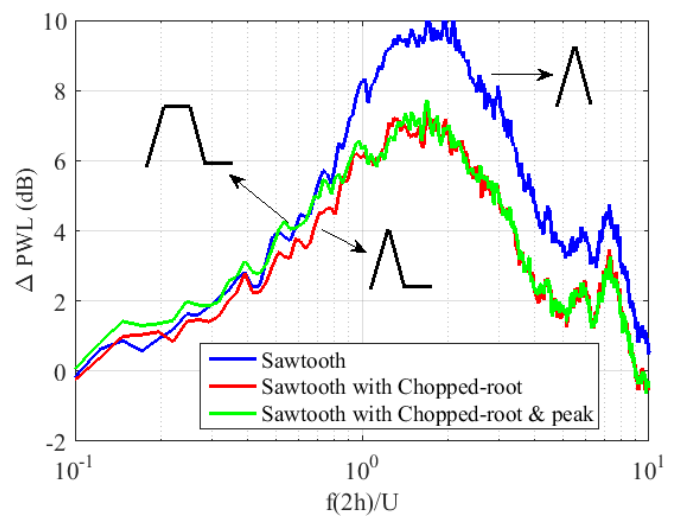

(b) Acoustic Performance

FIGURE 7.13: Chopped-root sawtooth serrations

At higher frequencies, $f(2 h) / U>1$, however, the level of noise reductions becomes sensitive to the presence of dominant sources, which tends to occur through the interaction between turbulent flow and rapid changes in leading edge profile, such as in the roots of single and double wavelength serrations (Chapters 4 and 5 respectively), and the roots either side of slits (Chapters 6 and 7). 



\section{Chapter 8}

\section{Conclusions and Future work}

Contents

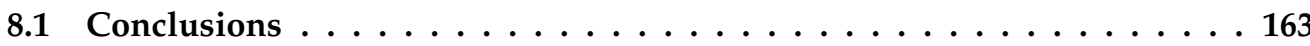

8.1 .1 General Conclusions . . . . . . . . . . . . . . . . . . . . 163

8.1 .2 Specific Conclusions . . . . . . . . . . . . . . . . . . 164

8.1.3 Comparison of different serration profiles . . . . . . . . . . . . 165

8.2 Future work . . . . . . . . . . . . . . . 166

\subsection{Conclusions}

This thesis presents a detailed experimental and numerical investigation into the effects of aerofoil geometry on turbulence-aerofoil interaction noise. The main focus of this thesis, however, is on the use of leading serrations to reduce the interaction noise.

\subsubsection{General Conclusions}

This thesis has shown that making modifications to an aerofoil geometry, such as thickness, leading edge radius and camber, to an aerofoil, produces reductions in noise of less than $1 \mathrm{~dB}$ at approach conditions. This conclusion has been validated experimentally on an isolated aerofoil and numerically in a 2D cascade. Clearly, therefore, significant levels of noise reductions required by, for example, Flightpath 2050, cannot be met through re-design of OGV aerofoil geometries. An alternative approach is therefore required.

This thesis has then focused on the use of leading edge serrations for reducing turbulenceleading edge interaction noise. Chapter 4 involves a detailed experimental investigation into the use of single wavelength serrations for reducing interaction noise. The optimum noise reduction performance has been identified corresponding to the case when adjacent root sources are only just excited incoherently. This has been shown to correspond to the optimum wavelength given by,

$$
\lambda_{0} \approx 4 \Lambda_{t}
$$

At this optimum condition, it has been shown that the sound power reduction level reduction is given by

$$
\Delta P W L(\omega)=10 \log _{10}(f h / U)+10
$$


Subsequent chapters have focused on the use of new leading edge profile designs aimed at improving the levels of noise reduction given by Eq. 8.2 afforded by single wavelength serrations. These new profiles have in common wavelengths that are smaller than the optimum wavelength given by Eq. 8.1 so that dominant sources on the leading edge are sufficiently coherent to interfere, which then leads to improved levels of noise reduction. At optimum conditions these new profiles have been shown to provide significantly higher levels of noise reductions compared to conventional single wavelength serrations. Simple analytic models have been developed to predict the additional levels of noise reduction that are shown to be in good general agreement with measured noise reduction data. The success of these simple models confirm that, despite the apparent complexity of turbulence-serrated leading edge interaction noise, the fundamental noise reduction mechanism is relatively simple.

\subsubsection{Specific Conclusions}

Some specific conclusions of this thesis may be summarised as follows:

1. Isolated flat plate analytical methods for predicting turbulence-leading edge interaction noise have been shown to be in very close agreement with the measured data.

2. Noise measurements made in a low speed wind tunnel $(M<0.3)$ provide a valid assessment of noise generation that may be extrapolated to higher flow speeds. Compressibility effects on interaction noise that occur at higher Mach numbers are therefore unimportant.

3. A detailed study into the effect of geometry variations to interaction noise make clear that these effects cannot be predicted based on a single length parameters, such as thickness or noise radius, such as has been attempted by some authors previously. It has been shown that noise predictions based on the non-dimensional frequency $f t / U$ provide a first approximation to predicting the effects on geometry. More accurate predictions require knowledge of the complete profile.

4. A major drawback of the flat plate model for cascades is that it doesn't account for camber effects which are shown to be significant in the case of turbulence-cascade interaction noise at the frequencies of interest.

5. A theoretical framework has been developed by which turbulence-cascade broadband interaction noise can be predicted in 2 dimensions using a linearized Navierstokes solver for the mean flow and a turbulence description involving a summation of Fourier vortical modes.

6. The effects of aerofoil thickness, stagger angle, camber and flow incidence have potentially large effect on individual modes in cascade interaction noise. However, when summed to compute overall broadband noise, the differences were generally negligible $(<1.5 \mathrm{~dB})$ at the realistic Mach numbers and frequencies of interest.

7. A new noise control principle has been developed and validated for the reduction of broadband interaction noise on aerofoils. The new control method is capable of providing substantially better noise reduction that the maximum noise reduction achievable on single-wavelength serration geometries.

8. Introducing serrations onto the aerofoil leading edge has been shown to produce significant reductions in self-noise of up to $3 \mathrm{~dB}$. 
9. New leading edge profiles (slitted profiles) has been developed to overcome the fundamental limitation of double-wavelength serrations whose performance was shown to be limited by the imperfect coherence between adjacent roots. A model has been developed to predict and understand the additional noise reductions due to slitted-root serration profiles.

10. Enhanced noise reductions has been demonstrated by a different family of leading edge profiles, such as a variable-slit profile, a chopped-peak profile. The profiles provide enhanced levels of noise reduction by either increasing the source strengths at the peak or by distributing the dominant root sources along the streamwise direction such that they interfere more effectively.

11. Finally, reduction in broadband interaction noise of up to $15 \mathrm{~dB}$ can be achieved by leading edge serrations. In most of the profiles considered, a self similarity property has been established which implies that these high levels of noise reduction are possible at all flow speeds.

This thesis concludes with a comparison of the noise control performance of the various leading edge profiles proposed.

\subsubsection{Comparison of different serration profiles}

Sound power reductions measured from all the serration profiles investigated in this thesis are now compared at the same serration wavelength $\lambda / c_{0}=0.1$, serration amplitude $2 h / c_{0}$ $=0.167$ and flow velocity $60 \mathrm{~m} / \mathrm{s}$. Note that all the serration profiles being compared are optimized to achieve best noise reductions, with the focus at low frequencies $(f h / U<1 / 2)$ where conventional single wavelength serrations perform poorly (cf Eq. 8.2).

A table summarizing the geometric parameters of each profile is shown below.

\begin{tabular}{c|c} 
Serration profile & dimensions \\
\hline Sinusoidal & $\lambda / c_{0}=0.1$ \\
\hline Sawtooth & $\lambda / c_{0}=0.1$ \\
\hline Double-wavelength & $\lambda_{1} / c_{0}=0.067, \lambda_{2} / c_{0}=0.133, \phi=\pi / 2$ \\
\hline Chopped-peak & $\lambda / c_{0}=0.1, l_{c} / \lambda=0.33$ \\
\hline Slitted-root & $h_{s} / c_{0}=0.21, \lambda / c_{0}=0.1, w / \lambda=0.13$ \\
\hline Variable-slit & $\lambda / c_{0}=0.2, a / \lambda=0.033, b / \lambda=0.167$ \\
\hline Slitted profile & $h_{s} / 2 h=1, w / \lambda=0.11, a / \lambda=0.89$ \\
\hline
\end{tabular}

TABLE 8.1: Leading edge serration profiles considered in this study for the same peak-to-root amplitude $h / c_{0}=0.167$

The spectral comparison in Figure 8.1 clearly shows the slitted profiles provides the greatest level of noise reduction over the entire frequency range where self-noise is negligible. Greatest noise reductions are therefore achieved when the two source strengths at either ends of the slit are equal at the tuned frequency. This balancing of source strengths is achieved by the careful choice of slit width. The variable slit serration can be seen to provide nearly the same levels of noise reduction at low and mid-frequencies but is significantly worse at higher frequencies compared to conventional sawtooth serrations.

The chopped-peak and double-wavelength serrations appear to provide similar levels of noise reduction at low frequencies. In these example, peak noise reductions are observed at 


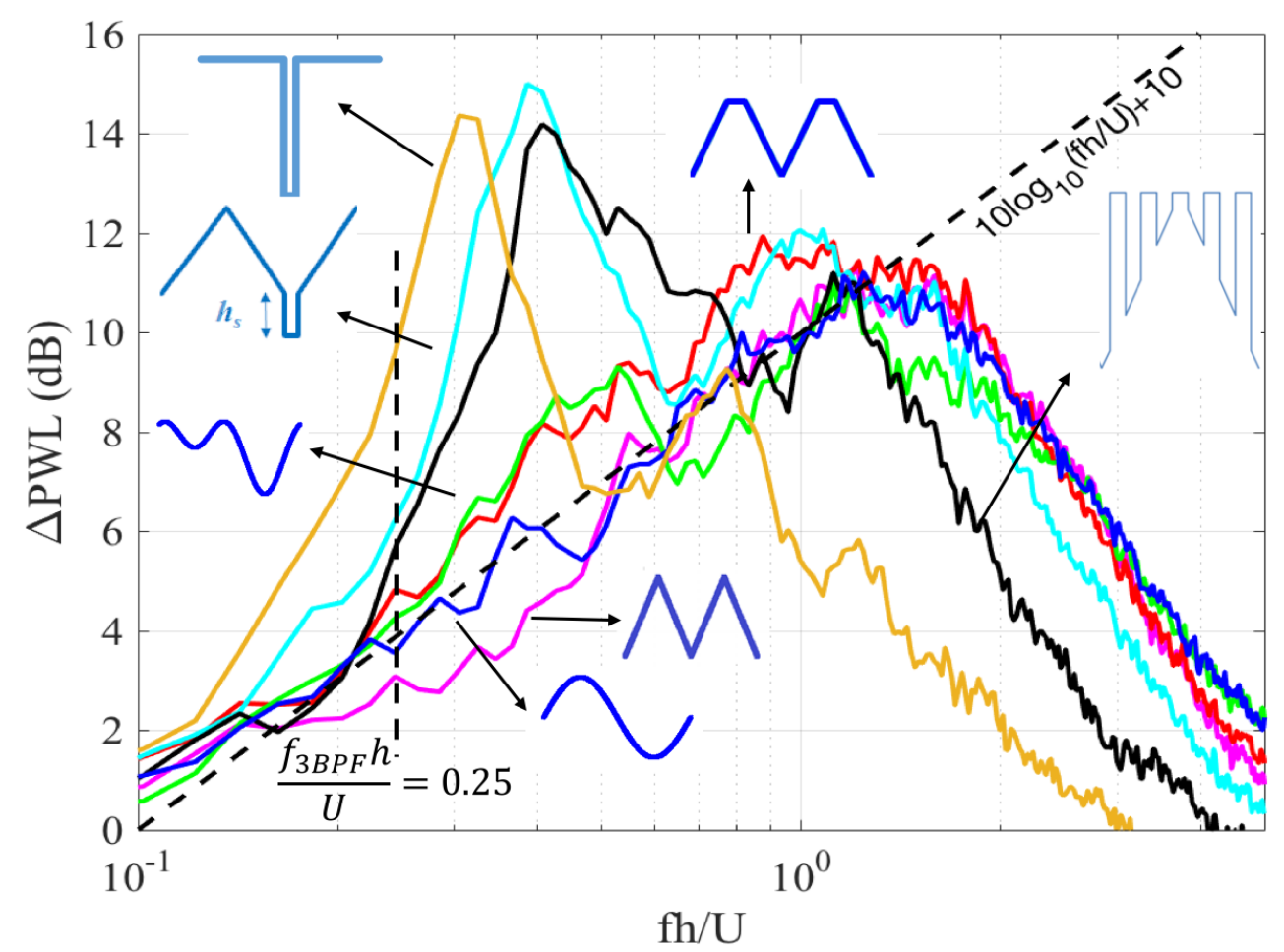

FIGURE 8.1: Acoustic performance of various serration geometries for a fixed peak-to-root distance $\left(h / c_{0}=0.167\right)$ at flow velocity of $60 \mathrm{~m} / \mathrm{s}$ (Black dashed: $\left.10 \log _{10}(f h / U)+10\right)$; Blue solid: Sinusoidal; Magenta solid: Sawtooth; Green solid: Double-wavelength; Red solid: Chopped-peak; cyan solid: Slitted-root;

Black solid: Variable-slit; Slitted profile: Orange.

mid frequencies with the chopped peak providing slightly better noise reduction by about $2 \mathrm{~dB}$ than the double wavelength serrations.

However, all of these profiles provide substantially better noise reductions by up to $10 \mathrm{~dB}$ than conventional single wavelength serrations.

\subsection{Future work}

The recommendations for future work are listed below:

1. To apply CFD methods to understand more clearly the noise reduction mechanism of the leading edge geometries proposed in this thesis.

2. To extend the parametric study on serration cut into flat plates to $3 \mathrm{D}$ aerofoils.

3. To investigate the aerodynamic performance of the leading edge serrations on 3D aerofoils.

4. To investigate the performance of leading edge serrations at high angles of attack and at post-stall conditions.

5. To develop a theoretical framework by for which turbulence-cascade broadband interaction noise can be predicted in 3 dimensions for realistic aerofoils. 
6. To incorporate the effect of camber in LINSUB to predict the upstream and downstream radiated power of turbulence-cascade interaction noise for flat plates.

7. Use of the RANS mean flow around the aerofoil leading edge to estimate the extent of gust distortion by using rapid distortion and panel method theory. This would help to develop a computational less expensive tool to predict the turbulence-aerofoil interaction noise.

8. To develop a combined leading and trailing edge serration for quieter low noise aerofoils. 



\section{Appendix A}

\section{Experimental setup}

This appendix describes the experimental facilities and acoustic measurement techniques used in the present thesis that focuses on measuring the Leading edge interaction noise generated in a turbulent flow. This section starts with ISVR's quite open-Jet wind tunnel facility which is used to perform acoustic measurement on airfoils. Then followed by flow characterizing techniques such as hot-wire anemometry which will help to characterize the turbulent at the nozzle exit and flow visualization techniques such as particle image velocitometry (PIV) to visualize the flow around the airfoil.

\section{A.1 Open-Jet wind tunnel}

Far field noise measurements were carried out at the ISVR's open-jet wind tunnel facility. Figure A.1 shows a photograph of the facility within the anechoic chamber of dimensions $8 m \times 8 m \times 8 m$. The walls are acoustically treated with glass wool wedges. The cut-off frequency of the chamber is about $80 \mathrm{~Hz}$. A detailed description of the facility is presented by Chong et al. (2008). To maintain two-dimensional flow around the aerofoil, side plates are mounted to the nozzle exit which will also support the aerofoil. The nozzle dimensions are $15 \mathrm{~cm} \times 45 \mathrm{~cm}$. Aerofoils are located $0.15 \mathrm{~m}$ downstream of the nozzle to ensure that the entire aerofoil is located well within the jet potential core, whose width is at least $12 \mathrm{~cm}$, as shown in figure 11a in Chong et al. (2008). As shown in Chong et al. (2008) the flow is two dimensional (no spanwise variation) to within an deviation of about $4 \%$. The maximum jet speed investigated in this study is $80 \mathrm{~m} / \mathrm{s}$. The background noise is atleast $10 \mathrm{~dB}$ below the airfoil noise, and the free stream turbulence (without turbulence grid) is less than $0.4 \%$ in the potential core of the nozzle.

In order to prevent tonal noise generation due to Tollmien-Schlichting waves convecting in the laminar boundary layer, and to ensure complete consistency between the different cases, the flow near the leading edge of the aerofoil was tripped to force transition to turbulence using a rough band of tape of width $1.25 \mathrm{~cm}$ located $16.6 \%$ of chord from the leading edge, on both suction and pressure sides. The tape has roughness of SS 100, corresponding to a surface roughness of $140 \mu \mathrm{m}$. Transition is forced by the use of trip tape, which is many orders of magnitude rougher than the aerofoil surface, and is therefore highly unlikely to affect transition. Previous noise measurements have indicated that self noise is insensitive to the method of tripping.

The mounting and airfoil models investigated in the present work is described in the respective chapters of the thesis. 


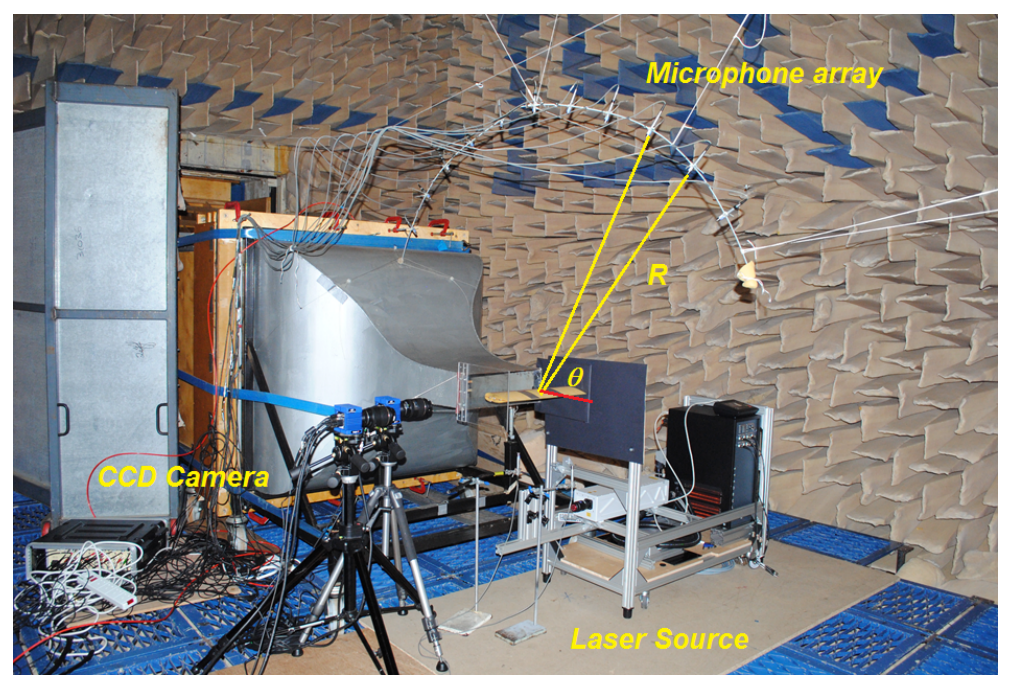

FIGURE A.1: Photograph of ISVR's Open-jet wind tunnel facility with Particle Image Velocimetry (PIV) setup (collaborated by AFM group, University of Southampton.

\section{A.2 Measurement techniques}

\section{A.2.1 Far-field noise measurement}

A.2 shows the distributed microphone array which is used to measure the far-field pressure. Far-field measurements were made by using 13 to 19, half-inch condenser microphones (B\&K type 4189) located radially at a distance of $1.2 \mathrm{~m}$ from the mid span of the airfoil having arc centre at airfoil leading edge. These microphones are placed at emission angles between $40^{\circ}$ and $140^{\circ}$ measured relative to the downstream jet axis. The microphones are connected to a amplifier which is manufactured in-house, and then to a National Instruments PXI 1042 chassis. The data acquisition is controlled via laptop which is connected via express card NI 8360. Three NI PXI-4472 Data Acquisition Cards are available which provided 24 channel to acquire the data at the same time. The available sampling rate per each channel is around $102.4 \mathrm{kS} / \mathrm{s}$. Measurements were carried for 10s duration at a sampling frequency of $50 \mathrm{kHz}$, and the noise spectra were calculated with a window size of 1024 data points corresponding to a frequency resolution of $48.83 \mathrm{~Hz}$ and a $B T$ product of about 500, which is sufficient to ensure negligible variance in the spectral estimate.

The acoustic pressure at the microphone locations were recorded at the mean flow velocities $(U)$ of 20,40, 60 and $80 \mathrm{~m} / \mathrm{s}\left(R e_{c}=206,500,413,000,620,000\right.$ and 826,000 respectively). Farfield microphones are calibrated using B\&K 4231 calibrator to get the correct sensitivities which might change slightly due to amplifier channel, amplified gain settings etc.

\section{A.2.2 Characterization of the jet with turbulence grid}

A bi-planar rectangular grid with overall dimensions of $630 \times 690 \mathrm{~mm}^{2}$ located in the contraction section of the nozzle was used to generate turbulence that is closely homogeneous and isotropic (shown in figure A.3). The grid was located $75 \mathrm{~cm}$ upstream of the nozzle exit. The velocity spectrum was measured using a hot wire at a single on-axis position $145 \mathrm{~mm}$ downstream from the nozzle exit. It is found to be in close agreement with the von-Karman spectrum for homogeneous and isotropic turbulence with a $2.5 \%$ turbulence intensity (TI) 


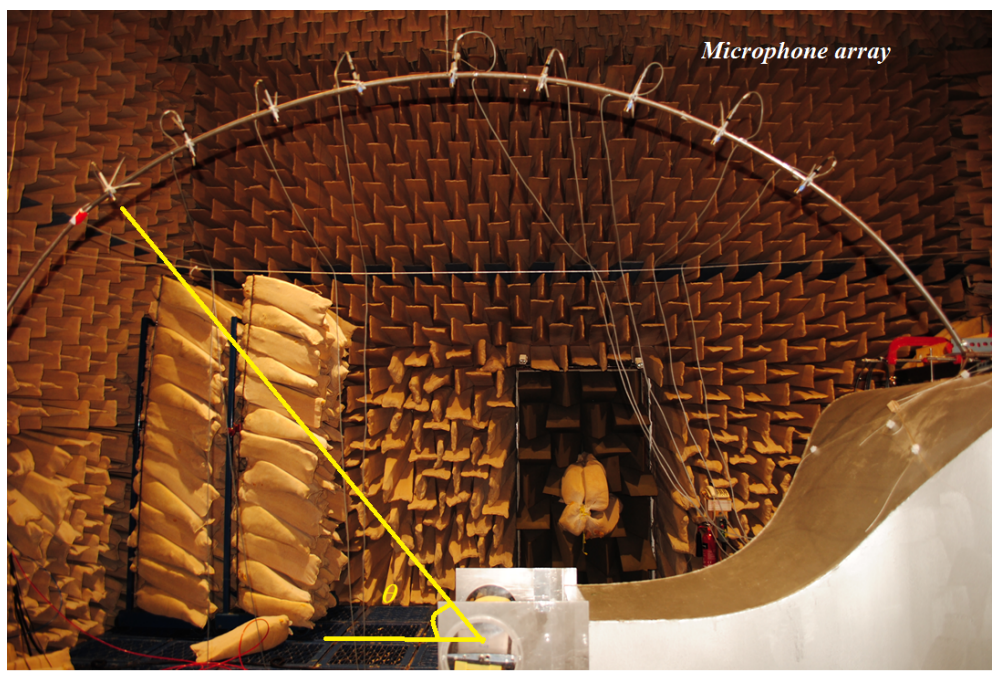

FIGURE A.2: Far-field measurements using microphone array.

and a $7.5 \mathrm{~mm}$ integral length scale. Bi-planar rectangular grid is designed based on Roach (1987).

To achieve larger integral length scale, a perforated grid has been designed based on Liu et al. (2004). Another grid of the same overall dimension but with multiple holes of $40 \mathrm{~mm}$ diameter was also used to generate homogeneous and isotropic turbulence but at a much larger length-scale of approximately $13.5 \mathrm{~mm}$ and a turbulence intensity of $4.5 \%$. The turbulence integral length scale was obtained by matching the theoretical spectra to the measured streamwise velocity spectra and dividing by two, assuming perfect isotropic turbulence. The integral length scale $\left(\Lambda_{t}\right)$ associated with the transverse velocity component (responsible for noise generation on a flat plate) was inferred from the streamwise length scale to be $3.75 \mathrm{~mm}$ and $6.75 \mathrm{~mm}$ respectively. A comparison of the two measured streamwise velocity spectra $\left(S_{u u}\right)$ together with the theoretical von-Karman spectra given by Equation A.1 are plotted in figure A.4, where close agreement is observed.

$$
\phi_{u u}^{V K}(f)=\frac{\overline{u^{\prime 2}} \Lambda_{u u}}{\pi U_{0}} \frac{1}{\left(1+\left(k_{x} / k_{e}\right)^{2}\right)^{5} / 6} k_{e}=\frac{\sqrt{(} \pi)}{\Lambda_{u u}} \frac{\Gamma(5 / 6)}{\Gamma(1 / 3)}
$$

where $\Lambda_{u u}$ is the integral length scale, $k_{x}=\omega / U_{0}$ is the stream-wise wavenumber, $T I=$ $\overline{u^{\prime}} / U_{0}$ is the turbulence intensity, $U_{0}$ is the flow velocity.

\section{A.2.3 Hot-wire measurement}

Boundary layer velocity profiles were acquired with hot-wire anemometry. A single hotwire probe (Dantec type 55P11) was driven using a standard constant temperature anemometry bridge (AA Lab System) operated at an over-heat ratio of 1.5. The hot-wire sensor was calibrated in the free-stream against a standard Pitot tube. The calibration function was a third-order polynomial that was fitted to the calibration data pairs (including the voltage at zero velocity). The preliminary wall position of the sensing element was obtained optically and was adjusted by fitting the near-wall data to a prescribed mean velocity distribution. To ensure convergence of mean statistics the hot-wire signals were acquired for a period of $60 \mathrm{~s}$ at sampling frequency of $20 \mathrm{kHz}$. A.5a shows the photograph of the setup. Calibration of hot-wire probe is carried out first. Voltage output from hot-wire probe are measured at 


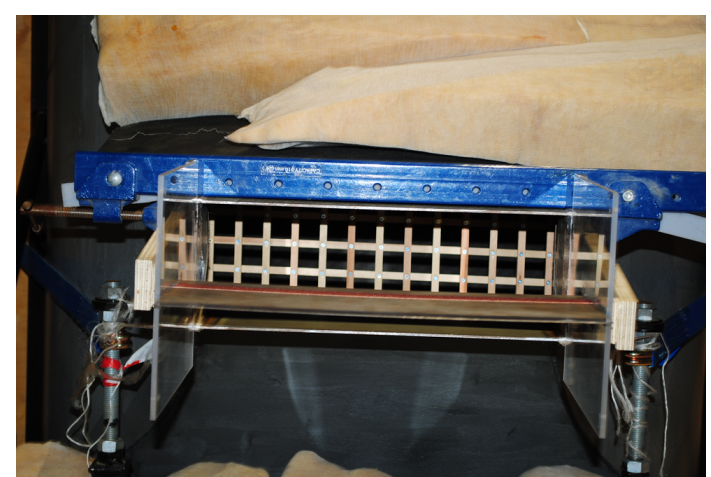

(a) Nozzle with turbulent grid.

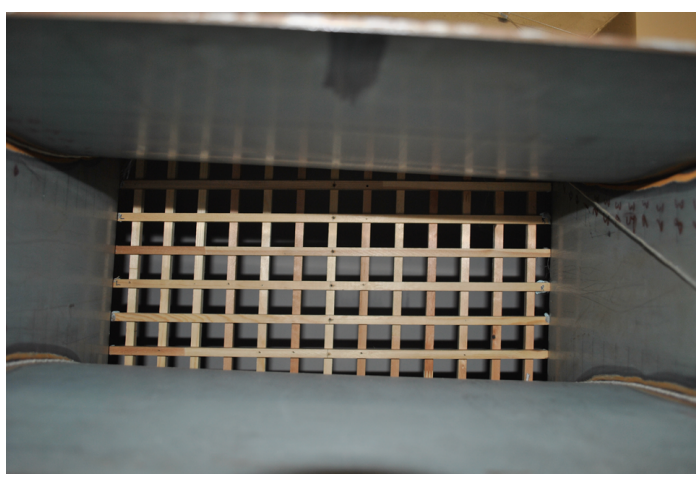

(b) Zoom-in view of grid inside nozzle

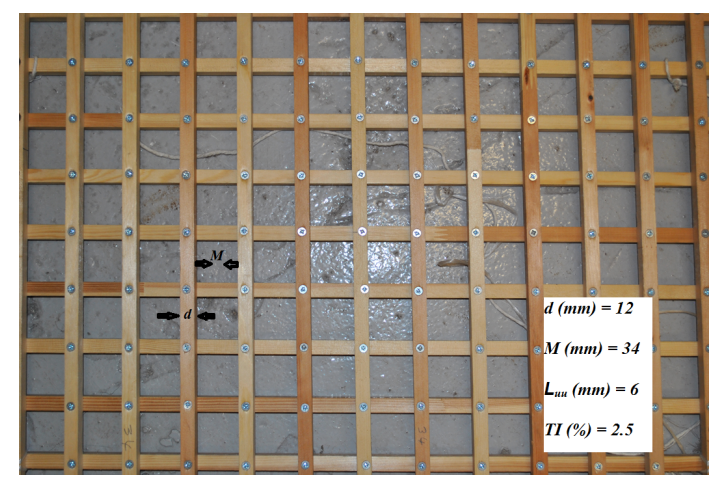

(c) Grid dimensions

FIGURE A.3: Location of turbulent grid inside the nozzle.

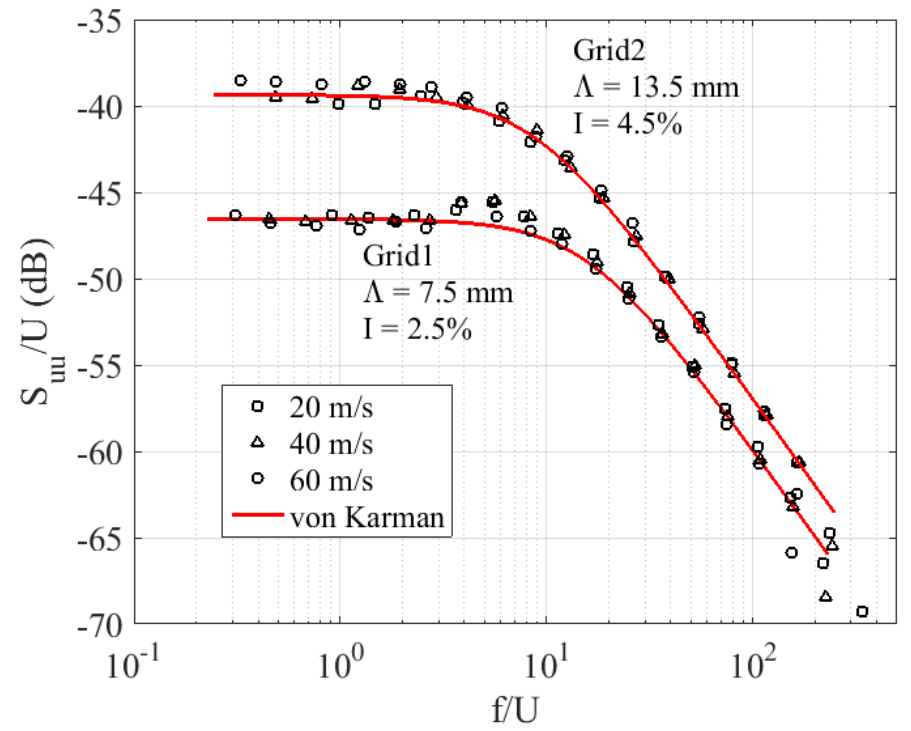

FIGURE A.4: Comparison between the measured axial velocity spectra and von-Karman 


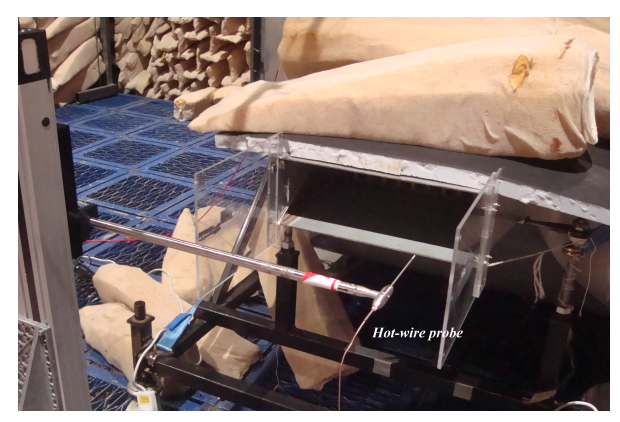

(a) A photogpragh of the hot-wire anemometry experimental setup.

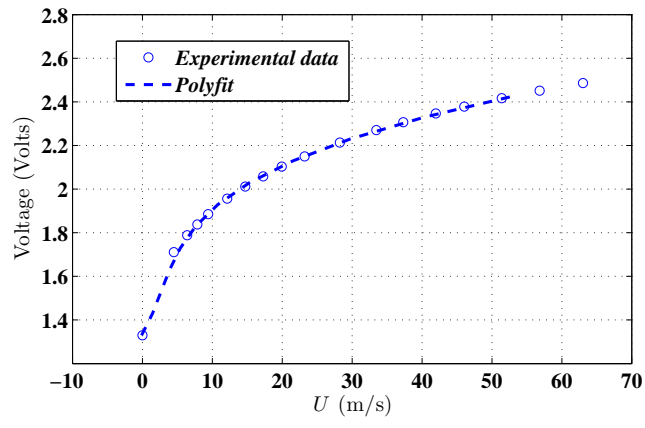

(b) Calibration of hot-wire probe

FIGURE A.5: Hot-wire anemometry setup \& calibration.

various jet velocities varying from 5 to $65 \mathrm{~m} / \mathrm{s}$. The jet velocities at each configuration of calibration procedure are measured with the help of static pitot tube which is connected to flow meter. A curve has been fitted for velocity versus voltage data to get the relation between them as shown in A.5b. This is later used to convert the voltage output from the hot-wire to the flow velocities in the real measurements.

\section{A.2.4 Particle Image Velocimetry (PIV) setup}

To assess whether the presence of leading serration on a realistic airfoil was detrimental to its aerodynamic behavior the flow field around the serrated NACA65 airfoil was investigated in detail using Particle Image Velocimitry (PIV). PIV is also used in the present work to visualize the flow around leading edge nose region around the airfoil as well as the development of boundary layer at the trailing edge of the airfoil. The arrangement of cameras and lasers in relation to the jet nozzle is shown in A.6. Two LaVision Imager-LX 29MP (megapixel) CCD cameras, fitted with lens having with a focal length of $100 \mathrm{~mm}$ and with an aperture of $\mathrm{f} 5.6$ are focused on the airfoil for two different field of views. One field of view is made to focus on the airfoil leading edge. The second focuses on the complete airfoil to observe the turbulent boundary layer and wake properties. The laser source is a Nd:YAG Laser Bernoulli 200-15 PIV which is focused into a thin laser sheet oriented parallel to the chord at position aligned with at the root and peak of the serrated airfoil as shown in A.6b. A fog machine (Magnum 1200) was placed at the inlet of the centrifugal fan. Homogeneous smoke then emerges from the jet nozzle and passed around the airfoil. Camera measurements were recorded at $1 \mathrm{~Hz}$, with an image pair separation time $(d t)$ of $20 \mu \mathrm{s}$. An average of 500 measurements were taken to obtain the mean velocity vectors around the airfoil. A photograph of the PIV setup in the anechoic wind tunnel is shown in the A.1.

\section{A.2.5 Aerodynamic measurements}

To evaluate the aerodynamic performance of the baseline and serrated airfoils, the vertical and horizontal forces acting on the airfoil were measured using a two-axis load cell (NOVATECH F314) which were mounted on either side of the airfoil as shown in figure A.7. Airfoils were directly mounted on the load-cell using a single shaft which minimizes the other reaction forces that could influence the lift and drag measurements. The force resolution of the load-cell is $0.1 \mathrm{~N}$ corresponding to a measurement error of less than $1 \%$. The 


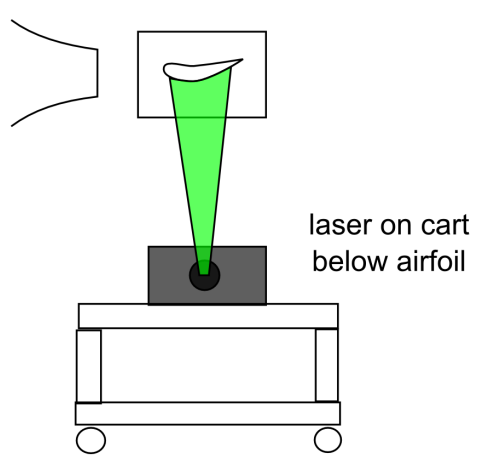

(a) Schematic of measurement procedure

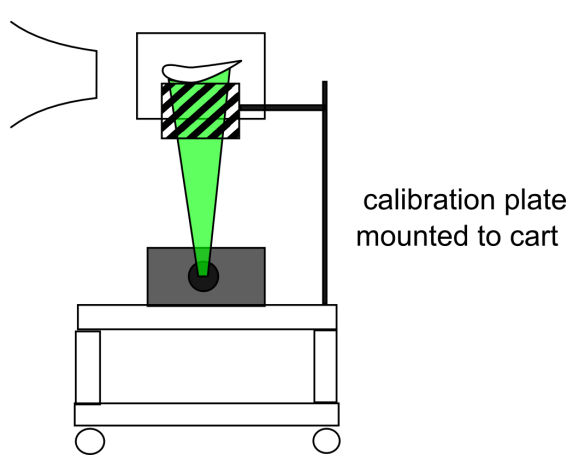

(b) Schematic of calibration procedure

FIGURE A.6: Schematic of the PIV setup.

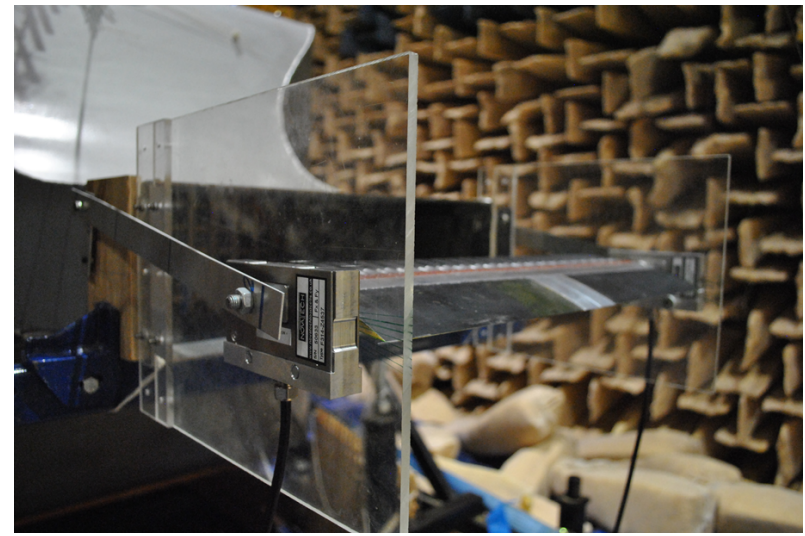

FIGURE A.7: Photograph of aerodynamic measurements in an open jet wind tunnel.

measurements were carried out at jet velocities of 20,40 and $60 \mathrm{~m} / \mathrm{s}$ and the geometric angle of attack were varied between $-2.5^{\circ}$ and $10^{\circ}$ corresponding to an effective angle of attack between $-0.7^{\circ}$ to $2.8^{\circ}$ due to jet deflection (Brooks et al. (1989)). The lift and drag was calculated by resolving the vertical and horizontal force components in the directions normal and parallel to the jet flow direction. Brooks et al. (1989) showed that in the presence of an aerofoil the flow from the open-jet wind tunnel is deflected downwards which does not occur in free air. The geometrical angle of attack is therefore corrected to obtain the effective angle of attack in free air. The lift and drag were calculated by resolving the vertical and horizontal force components in the directions normal and parallel to the jet deflection direction which was estimated from the difference between the geometric and effective angles of attack. The effects on lift and drag due to corner effects are believed to be relatively small due to the relatively large aerofoil aspect ratio 3:1 used in this study, and in any case only the relative difference in lift and drag between baseline and serrations are of interest in this thesis.

\section{A.3 Measuring TE noise and LE noise}

This Section presents typical measurements of aerofoil TE noise and LE noise. Separate measurements of the total noise radiation (i.e., the sum of LE, TE and background noise), the sum of self noise and background noise (by removing the turbulent grid), background noise spectra alone with and without turbulence grid (without aerofoil), and jet near-field data are 


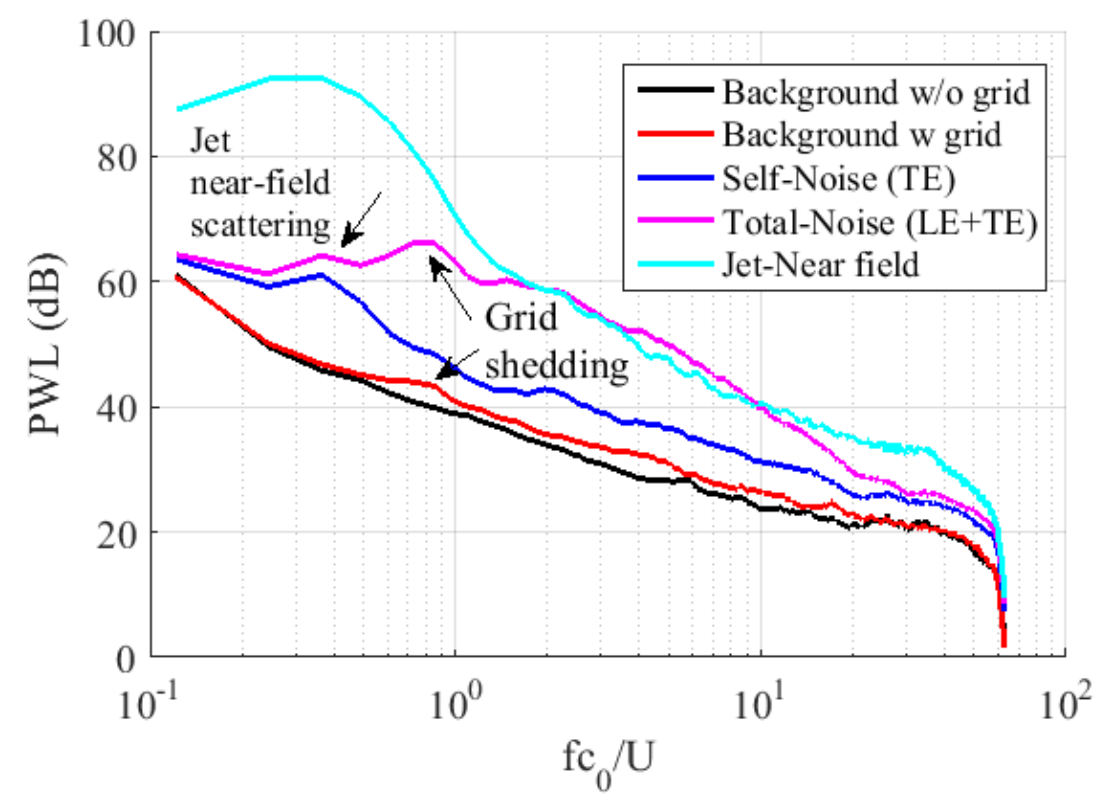

FIGURE A.8: Typical far-field LE interaction noise and TE noise measured on the isolated NACA65(12) aerofoil, at $U_{0}=60 \mathrm{~m} / \mathrm{s}$ and $0^{\circ}$ AoA.

plotted in the figure A. 8 at a jet velocity of $60 \mathrm{~m} / \mathrm{s}$. It is observed that the LE noise dominates the TE noise till non-dimensional frequency $f c_{0} / U 15$. Below $f c_{0} / U$ of 0.25 , the spectra is dominated by jet noise as also this is seen in the background noise spectra. Between $f c_{0} / U$ of 0.25 and 0.5 , we see an additional noise source which is not seen in far-field background noise spectra. This can be due to near-field scattering of the jet hydrodynamic field with the LE or TE of the aerofoil. A near-field jet spectra is also plotted in the figure A.8 where the peak frequency of the near-field spectra falls in between $f c_{0} / U$ of 0.25 and 0.5 . This nearfield is not seen in the background data as it decays before it reaches the far-field. Additional noise source around non-dimensional frequency $f c_{0} / U$ of 0.75 is observed which matches with the velocity spectral hump as shown in the figure A.4. This is only seen for the case of bi-planar grid and this can be due to vortex shedding from the bar of the turbulence grid. This additional hump is also seen in the background noise data with turbulence grid as shown in the figure A.8. In summary, LE is dominates in the frequency range of $f c_{0} / U$ of 1 to 15 . 



\section{Appendix B}

\section{Analytical Flat plate solution}

The analytic solution for turbulence interacting with flat plate is presented in this Appendix.

\section{B.1 Amiet's flat plate turbulence-aerofoil interaction model}

\section{B.1.1 Model formulation}

The problem of predicting the sound pressure radiated from an isolated flat plate in a turbulent stream was addressed by Amiet (1975). This section presents an expression for the pressure power spectral density of the noise generated due to interaction of turbulent gust on an aerofoil. The following assumptions considered in the present analysis.

1. Turbulence is assumed to be frozen in the present analysis.

2. Jet free stream is extended to infinity i.e. the analysis doesn't consider shear layer.

3. The observer is located in far-field of the aerofoil.

4. Source is approximated as distributed dipole sources of equal strength.

5. Thickness of aerofoil is negligible (flat plate).

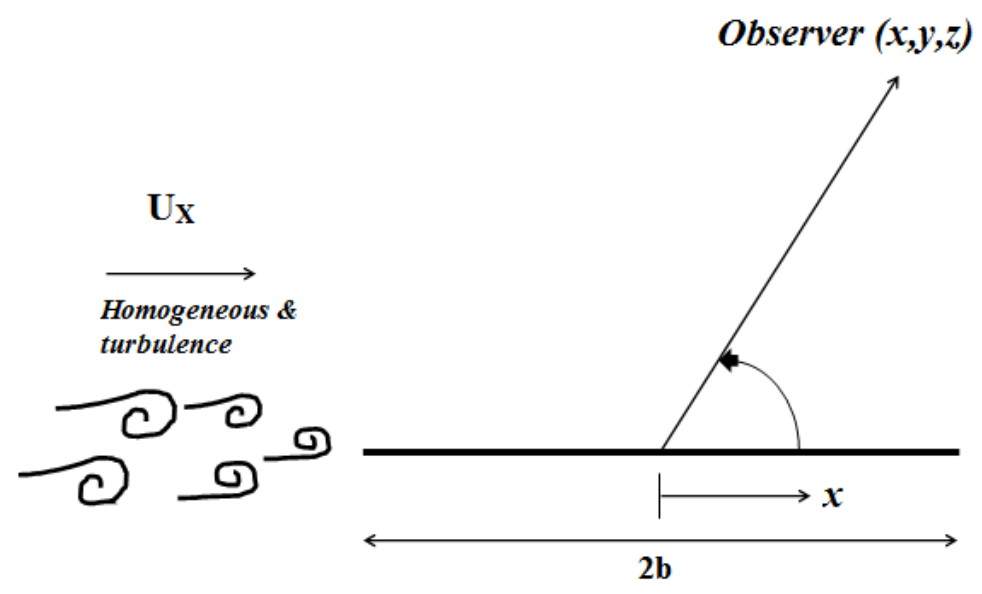

FIGURE B.1: Configuration of the flat plate interacting with a harmonic gust. 
Assuming, $\Delta P$, as the pressure jump across flat plate caused due to sinusoidal gust $\left(w_{g}\right)$ interacting the flat plate in turbulent flow. The incoming gust is of the form

$$
w_{g}=w_{0} e^{i k_{x}\left(x-U_{x} t\right)+k_{y} y}
$$

and the pressure jump can be expressed as

$$
\Delta P(x, y, t)=2 \pi \rho_{0} U b w_{0} g\left(x, k_{x}, k_{y}\right) e^{i\left(k_{y} y-k_{x} U_{x} t\right)}
$$

where $U_{x}$ is the mean flow in $x$ direction (along the chord), $k_{x}$ and $k_{y}$ are the turbulence wavenumbers along the chord-wise and span-wise direction, $b=c_{0} / 2$ is the semi-chord length, $g\left(x, k_{x}, k_{y}\right)$ is the transfer function between turbulent velocity and aerofoil pressure jump. The unsteady pressure jump in the frequency domain is obtained by Fourier transform of Equation B.2 and is given by (Amiet (1975)):

$$
\Delta P_{T}(x, y, \omega)=2 \pi \rho_{0} b \int_{-\infty}^{+\infty} W\left(K_{x}, k_{y}\right) g\left(x, K_{x}, k_{y}\right) e^{i k_{y} y} d k_{y}
$$

where $K_{x}=\omega / U_{x}$ Due to randomness of turbulence, the cross-power spectral density of the pressure jump is given expressed as the ensemble average of pressure jump at two points on the aerofoil surface. The final expression for cross-PSD is given as (Amiet (1975)):

$$
S_{Q Q}\left(x_{1}, x_{2}, \eta, \omega\right)=\left(2 \pi \rho_{0} b\right)^{2} U_{x} \int_{-\infty}^{+\infty} g^{*}\left(x_{1}, K_{x}, k_{y}\right) g\left(x_{1}, K_{x}, k_{y}\right) \phi_{w w}\left(K_{x}, k_{y}\right) e^{i k_{y} \eta} d k_{y}
$$

where $\eta=y_{2}-y_{1}, x_{1}$ and $x_{2}$ are the two point coordinates on the aerofoil surface. $\phi_{w w}\left(k_{x}, k_{y}\right)$ is the two-dimensional wavenumber spectrum of the transverse turbulence velocity component. von-Karman and the Liepmann models are the two widely used homogeneous and isotropic turbulence velocity spectra. The $2 \mathrm{D}$ Liepmann turbulence velocity spectra is given by

$$
\phi_{w w}\left(k_{x}, k_{y}\right)=\frac{3 \overline{w^{2}} \Lambda^{4}}{4 \pi} \frac{k_{x}^{2}+k_{y}^{2}}{\left[1+\Lambda^{2}\left(k_{x}^{2}+k_{y}^{2}\right)\right]^{5 / 2}}
$$

where $\overline{w^{2}}$ is the mean square turbulent upwash velocity and $\Lambda$ is the turbulence integral length scale The far-field pressure is related to the pressure jump across the aerofoil by approximating the distributing dipoles over the aerofoil surface whose strength is equal to the pressure jump. The far-field radiated sound generated by a point force on strength $\Delta P_{T}\left(x_{0}, y_{0}, \omega\right)$ is given by

$$
P\left(x, y, z, \omega ; x_{0}, y_{0}\right)=\frac{i \omega z \Delta P_{T}\left(x_{0}, y_{0}, \omega\right)}{4 \pi a S_{0}^{2}} e^{i \omega\left[t+\frac{M\left(x-x_{0}\right)-S_{0}}{a \beta^{2}}+\frac{x x_{0}+y y_{0} \beta^{2}}{a \beta^{2} S_{0}}\right]}
$$

where $k$ is the acoustic wavenumber given by $\omega / a$ and $a$ is sound speed

The far-field PSD is related to cross-power spectral density of the pressure jump and the final expression for the far field power spectral density (PSD) due to a flat plate interacting 
with isotropic turbulence is given below: [Roger (2010); Amiet (1975)].

$S_{p p}(x, y, z, \omega)=\left(\frac{k \rho b z}{S_{0}^{2}}\right)^{2} \pi U_{x} \frac{L}{2} \int_{-\infty}^{+\infty}\left[\Phi_{w w}\left(\frac{\omega}{U_{x}}, k_{y}\right)\left|L_{T I}\left(x, \frac{\omega}{U_{x}}, k_{y}\right)\right|{ }^{2} \frac{\sin ^{2}\left[\left(\frac{k y}{S_{0}}+k_{y}\right) \frac{L}{2}\right]}{\pi\left(\frac{k y}{S_{0}}+k_{y}\right)^{2} \frac{L}{2}}\right] d k_{y}$

$S_{0}=\left(x^{2}+\beta^{2}\left(y^{2}+z^{2}\right)\right)$ is the convective corrected distance, $L$ is the span length, $b$ is the semi-chord length, and $L_{T I}$ is a radiation function which takes different forms for subcritical and supercritical gusts which are given in Roger (2010).

\section{Two-dimensional formulation}

The derived far-field PSD can be simplified into two-dimensional equation considering the aerofoils as infinite-span. Equation B.7 can be simplified using a $L \rightarrow \infty$. This approximation results in

$$
\lim _{L \rightarrow \infty} \frac{\sin ^{2}\left[\left(\frac{k y}{S_{0}}+k_{y}\right) \frac{L}{2}\right]}{\pi\left(\frac{k y}{S_{0}}+k_{y}\right)^{2} \frac{L}{2}}=\lim _{L \rightarrow \infty} \frac{L}{\pi}\left(\operatorname{sinc}^{2}\left[\left(\frac{k y}{S_{0}}+k_{y}\right)\right)=\delta\left(\frac{k y}{S_{0}}+k_{y}\right)(\mathrm{B} .8)\right.
$$

Using the properties of delta function and substituting in Equation B.7 yields an expression far-field PSD due to turbulence-aerofoil interaction noise. As the aerofoil span is infinite, the simpler case is the observer in $y=0$ plane. This simplifies the far-field PSD equation as below:

$$
S_{p p}(x, 0, z, \omega)=\left(\frac{\omega z \rho_{0} b}{a S_{0}^{2}}\right)^{2} \pi U \frac{L}{2}\left|L_{T I}\left(x, K_{x}, 0\right)\right|^{2} \Phi_{w w}\left(K_{x}, 0\right)
$$

The sound pressure spectral comparison between amiet's 2D \& 3D models using Equations B.7 and B.9 are presently in Figure B.2 for varying $L / 2 b$ ratios. It has shown from figure B.2, with the increase of $L / 2 b$, the infinite span approximation holds good for the case of two-dimensional model. Significant difference are observed at low frequencies between $2 \mathrm{D}$ and $3 \mathrm{D}$ models at low $L / 2 b$ ratios which is due to infinite span approximation. Below are the parameters considered for the analysis. $M=U_{x} / a=0.261 ; c_{0}=2 b=0.233 \mathrm{~m}$; Turbulent intensity, $I=\sqrt{\frac{\overline{u^{2}}}{U_{x}^{2}}}=4.4 \%$; Integral length scale $\Lambda=0.03 \mathrm{~m}$; Observer location $(\mathrm{x}, \mathrm{y}, \mathrm{z})=(0,0,2.25) ; L / 2 b=0.5,1,2,4,8$.

This can also be interpreted as the parallel gust wavenumber i.e. $k_{y}=0$ are the dominant wavenumbers contribute to sound. The contributions from other wavenumber components to the total sound power radiation can seen in the section B.2.

\section{B.2 Importance of subcritical and supercritical gust frequencies}

$L_{T I}$ in the Equations B.7 and B.9 is the radiation term where the closed form solutions includes both the subcritical and supercritical gusts as given by Roger (2010). The solution is divided into two terms $\left(L_{1} \& L_{2}\right)$ : one solution is obtained assuming the aerofoil of infinite chord without trailing edge noise and second solutions assumes semi-infinite aerofoil 


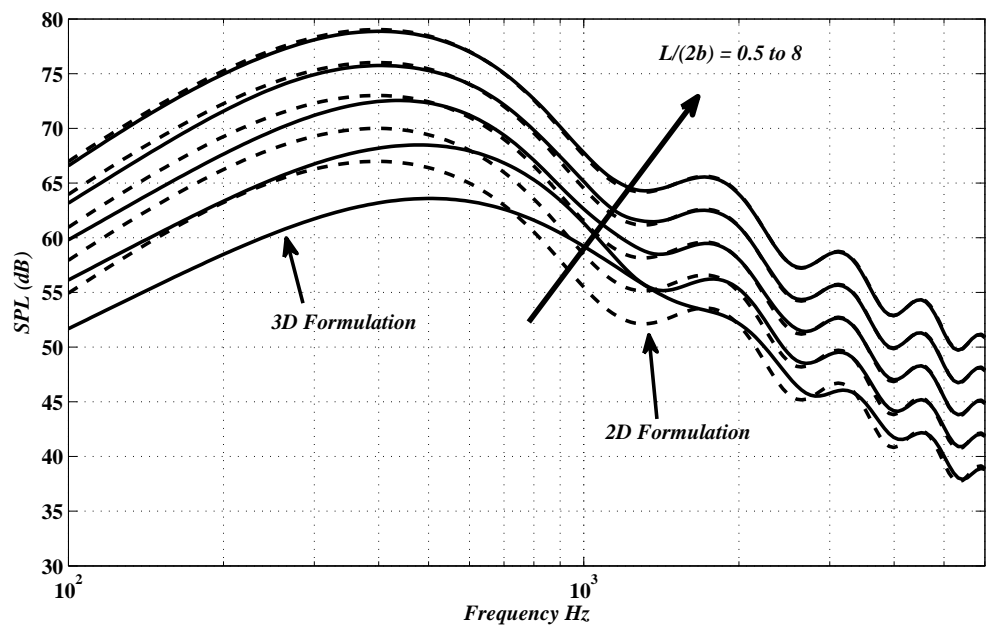

FIGURE B.2: Sound pressure spectral comparison between amiet's 2D \& 3D turbulent-aerofoil interaction model for varying $L / 2 b$.

with trailing edge and no leading edge. If the gust interaction point with the aerofoil moves faster than speed of sound, the gusts are known as supercritical gust which radiate to farfield whereas the interaction point moves slower than speed of sound then these gusts are know as subcritical gusts producing evanescent waves which will decay at short distance. These contributions are studied in the present section which would help to understand the frequency range of analysis which would save in computational time. The details expressions of these solutions for subcritical and supercritical are as below (Roger (2010)):

In case of subcritical gust when the Graham's parameter $\Theta_{0}=k_{x}^{*} M_{0} /\left(\beta k_{y}^{*}\right)$ is smaller than 1.

$$
\begin{aligned}
& L_{1}=-\frac{1}{\pi} \sqrt{\frac{2}{\left(k_{x}^{*}+i \beta^{2} \overline{\kappa^{\prime}}\right) i \Theta_{3}}} e^{-i \Theta_{2}} E\left[2 i \Theta_{3}\right] \\
& L_{2}=\frac{e^{-i \Theta_{2}}}{\pi \sqrt{2 \pi\left(k_{x}^{*}+i \beta^{2} \overline{\kappa^{\prime}}\right) \Theta_{3}}} \\
& \times\left\{1-e^{-2 \Theta_{3}}-\operatorname{erf}\left(\sqrt{4 \overline{\kappa^{\prime}}}\right)+2 e^{-2 \Theta_{3}} \sqrt{\frac{\overline{\kappa^{\prime}}}{i \overline{\kappa^{\prime}}+\bar{\mu} x / S_{0}}} E\left[2\left(i \overline{\kappa^{\prime}}+\bar{\mu} x / S_{0}\right)\right]\right\}
\end{aligned}
$$

where $\Theta_{3}=\bar{\mu}\left(M-x S_{0}\right)-\frac{\pi}{4}, \Theta_{3}=\overline{\kappa^{\prime}}+i \bar{\mu} \frac{x}{S_{0}}, \overline{\kappa^{\prime}}=\sqrt{\frac{k_{y}^{* 2}}{\beta^{2}}-\bar{\mu}^{2}},{\overline{\kappa^{\prime}}}^{2}=\bar{\mu}^{2}\left(\frac{1}{\Theta_{0}^{2}}\right), \bar{\mu}=\frac{k b}{2 \beta^{2}}=$ $\frac{k_{x}^{*} M}{\beta^{2}}, k_{j}^{*}=\frac{k_{j} b}{2}$

where $E$ is the Fresnel integral: $E(\zeta)=\int_{0}^{\zeta} \frac{e^{i t}}{\sqrt{2 \pi t}} d t$

In case of supercritical gust when the graham's parameter $\Theta_{0}=k_{x}^{*} M_{0} /\left(\beta k_{y}^{*}\right)$ is greater than 1 and ${\overline{\kappa^{\prime}}}^{2}<0$ and given by:

$$
L_{1}=-\frac{1}{\pi} \sqrt{\frac{2}{\left(k_{x}^{*}+\beta^{2} \overline{\kappa^{\prime}}\right) i \Theta_{4}}} e^{-i \Theta_{2}} E\left[2 \Theta_{4}\right]
$$




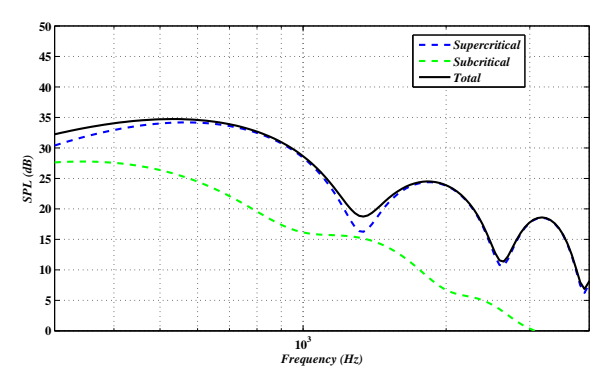

(a) $150^{\circ}$ microphonelocation

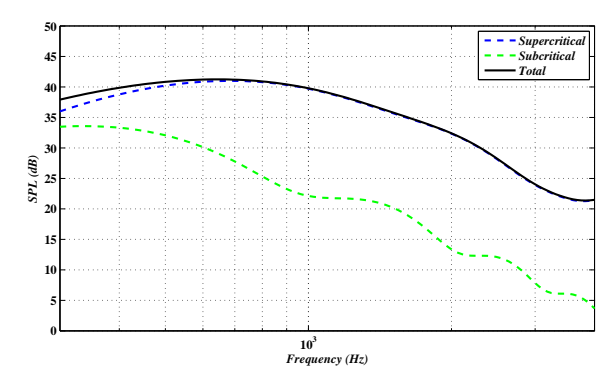

(b) $70^{\circ}$ microphonelocation

FIGURE B.3: Contribution of supercritical and supercritical gust to total turbulent-aerofoil interaction noise $\left(c_{0}=2 b=0.1 \mathrm{~m}, U=40 \mathrm{~m} / \mathrm{s}, I=\right.$ $5 \%, \Lambda=0.009 m)$.

$$
\begin{aligned}
L_{2}= & \frac{e^{-i \Theta_{2}}}{\pi \Theta_{4} \sqrt{2 \pi\left(k_{x}^{*}+\beta^{2} \bar{\kappa}\right)}} \\
& \times\left\{i \left(1-e^{2 i \Theta_{4}}-(1+i)\left[E(4 \bar{\kappa})-2 e^{-2 i \Theta_{4}} \sqrt{\frac{\bar{\kappa}}{\bar{\kappa}+\bar{\mu} x / S_{0}}} E\left[2\left(\bar{\kappa}+\bar{\mu} x / S_{0}\right]\right]\right)\right.\right.
\end{aligned}
$$

where $\Theta_{4}=\bar{\kappa}-\bar{\mu} \frac{x}{S_{0}}$

Figure B.3a and b shows the contributions from supercritical and subcritical gust wavenumbers in the total turbulent-aerofoil interaction noise. Gust parallel to chord $\left(k_{y}=0\right)$ is the efficient sound producer than compared to skewed gusts. As skewed gusts vary sinusoidally along the span there is an substantial cancellations and hence contribute significantly lesser. Figure B.3a and $\mathrm{b}$ confirms the similar observation, the contributions from subcritical gusts are negligible except for the low frequencies. The microphone position which is away from normal direction to the aerofoil have more contribution from subcritical gusts than compared to microphone position closer to normal axis to aerofoil. 



\section{Appendix C}

\section{HYDRA Setup}

\section{C.1 Process Map}

Figure C.1 gives an overview of the hydra user suite. The source for this appendix is Rolls Royce plc HYDRA user manual. The main elements of this suite are: (a) JM52 for preprocessing mesh files and setting up hydra input files; (b) JM56 for generating multi-grid levels and multi-passage application and (c) JL09 for post-processing and extracting quantitative data from unstructured mesh solutions (d) JM51 for non-linear, linear Navier-Stoke solvers.

\section{C.1.1 Meshing using PADRAM}

The current approach uses Rolls Royce plc meshing tool named PADRAM. PADRAM stands for Parametric Design and Rapid Meshing. As the name indicates, it is a CFD mesh generation tool designed for turbo-machinery components as well as a design system providing a rich set of parametrized design space to alter a given configuration. Generation of Structured, Unstructured and Hybrid (combination of both structured and un-structured) meshes are possible using PADRAM.

PADRAM is capable of creating a $2 \mathrm{D}$, quasi-3D, 3D, single passage, multi-passage meshes suitable for viscous and inviscid CFD calculations. The meshes are based on $\mathrm{H}-\mathrm{O}-\mathrm{H}$ multiblocks which are currently preprocessed by JM52 which write the input files for the HYDRA CFD code. The O-mesh is primarily used for the blade and in particular should be extended to capture the viscous region of the aerofoil. The H-mesh is used in the passages to link the O-mesh to the periodic boundaries and is used for the upstream and the downstream blocks.

\section{C.1.2 JM51}

Within Rolls-Royce plc. HYRA is released under the system name jm51. The hydra source code compiles a number of different executables, such as the serial and parallel versions of the non-linear, linear and adjoint solvers. JM51 have different executables for parallel version, linear and every adjoint solvers versions. 


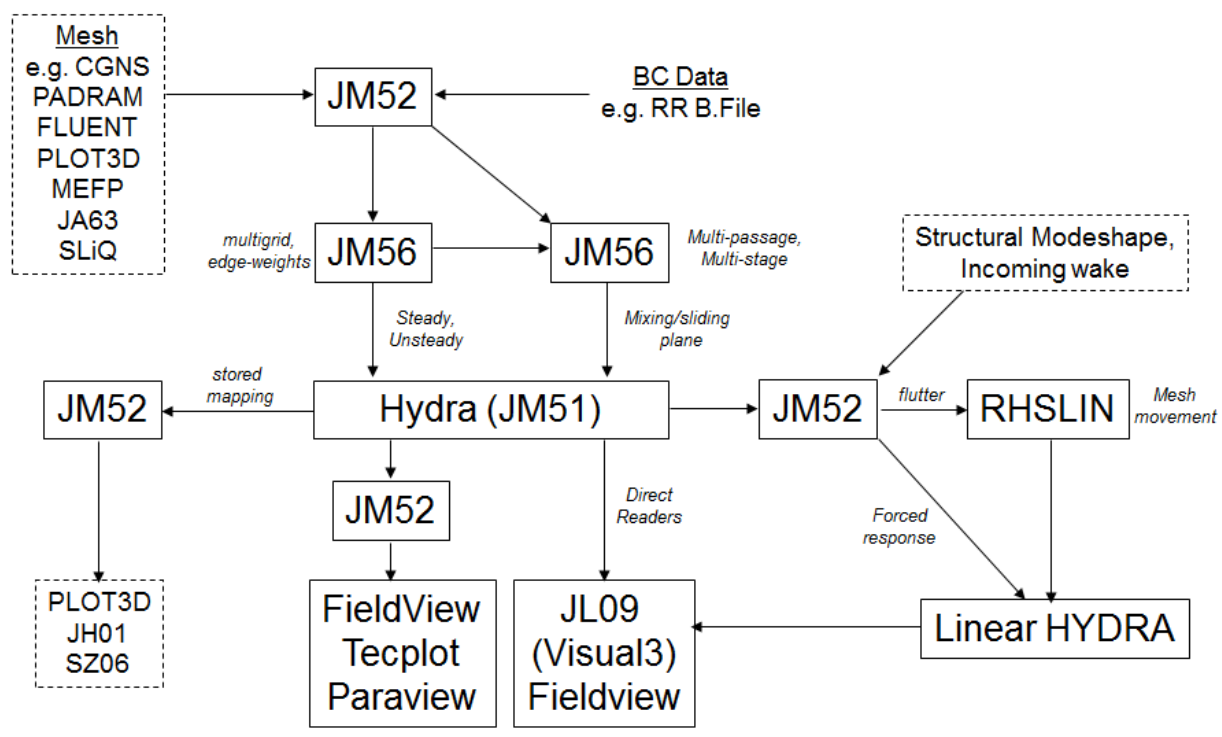

FIGURE C.1: Process Map (Courtesy: RR Hydra Manual)

\section{C.1.3 JM52}

JM52 is the primary pre-processing tool for the HYDRA suite. The program may be run interactively using the Graphical User Interface (GUI) or in script mode, which enables commands to be defined in a simple text file, which controls the execution of the program written using a simple scripting command structure. The scripting option is particularly powerful for driving the HYDRA calculations through a job scheduling script. The main function of JM52 is to translate meshes from a number of in-house and commercial mesh formats into HYDRA format for subsequent use in JM51 and JM56. The mesh can be checked using the JM52 diagnostic checks. These diagnostics check for negative cell volumes, cell degeneracies (i.e. cells with multiple entries of the same node index in their connectivity table), mesh periodicity (for periodic boundary cases only), cell connectivity (i.e. all cells are a valid part of the mesh having only one neighboring cell or boundary face for each individual cell face) and for point redundancy.

JM52 may also be used to initialize the flow data. A number of different options are available; a simple 1D axial profile defined by the user, a uniform flow condition defined by the user, linear interpolation from an existing SWIPE Boundary Conditions file or by reading an existing flow solution from one of the data formats defined above. JM52 is used to define the boundary conditions for the computation. This includes the definition of the boundary surface types as inviscid or viscous walls, inflow or outflow, periodic surfaces, etc.. Boundary conditions such as inflow and outflow boundary profiles can also be defined or may be taken from previously generated boundary condition files or directly from SWIPE Boundary Condition data.

\section{C.1.4 JM56}

For all meshes, JM56 is used to compute:

- Derived connectivity data, such as edge connectivity and edge weights, needed by hydra.

- Sequence of coarser meshes for the multigrid solver. 
The JM56 program is also the cornerstone of the HYDRA multistage/multi-component system. JM56s main functions, at present, are to build multipassage and multistage meshes for steady mixing plane and unsteady sliding plane calculations; and, to generate meshes for treated casings. Mesh and flow solution files for single row, single passage HYDRA computations can be imported into JM56 and joined through a simple series of steps using the JM56 Graphical User Interface (GUI).

\section{C.2 Stability of Linear Unsteady solver}

The linearized flow equations can be viewed as a large complex system of the form $A x=b$, in which $A$ is the linearization matrix, $x$ is the unknown complex linear flow field and $b$ is the right-hand-side depending on the displacement and velocity fields (flutter analyses) or the prescribed gusts at the far field boundaries (forced response or acoustic analysis). There are two alternative schemes available in hydra for recovering the stability of the linear unsteady solver: the Generalized Minimal Residual (GMRES) algorithm and the Recursive Projection Method (RPM).

\section{C.2.1 The Generalized Minimal Residual algorithm}

The Generalized Minimum Residual algorithm (GMRES) stabilizes the linear iterative scheme by constructing a sequence of mutually orthogonal basis vectors. Each basis vector is stored and as each new basis vector is added, the error components of the linear system within the space spanned by the available basis vectors is minimized. By construction, the residual of the linear system must decrease monotonically as new basis vectors are added.

\section{C.2.2 The Recursive Projection Method}

The Recursive Projection Method (RPM) is an iterative algorithm well suited for the solution of linear systems in the presence of outliers, that performs particularly well when the number of outliers is not too large.

\section{C.3 Grid definition and setup}

The numerical approach outlined above is implemented on non-staggered flat plate airfoils by way of validation against the LINSUB solution. As discussed in section 3.5.1, figure 3.3 shows the $2 \mathrm{D}$ section of the computational domain. The mesh is uniformly space except at the leading and trailing edge of the flat plate. A quantitative mesh refinement study has been carried out to quantify the errors due to dissipation and dispersion.

\section{Grid sensitivity studies}

A mesh sensitivity has been performed on flat plate to ensure the minimum dissipation for a given minimum acoustic propagating wavelength. figure C.2a plots the scattered acoustic pressure against number of points per wavelength $(c / f)$. The mesh sensitivity studies has been perform for the maximum angular frequency of interest $\omega=30000$ and for all these cases the mesh near the leading is sufficient very fine to capture the maximum unsteady pressure at the leading edge. A reasonable convergence has been seen above the 150 points 


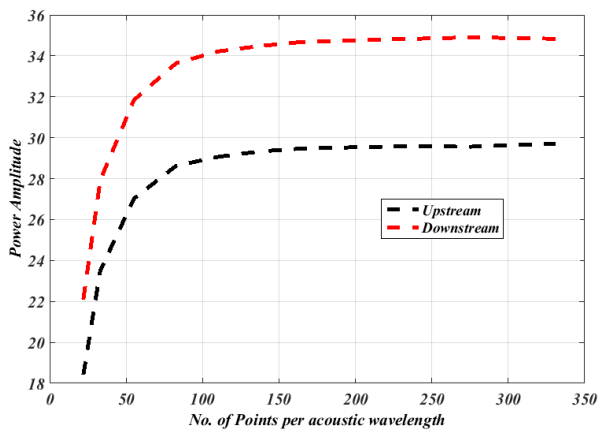

(a) Pressure Amplitude

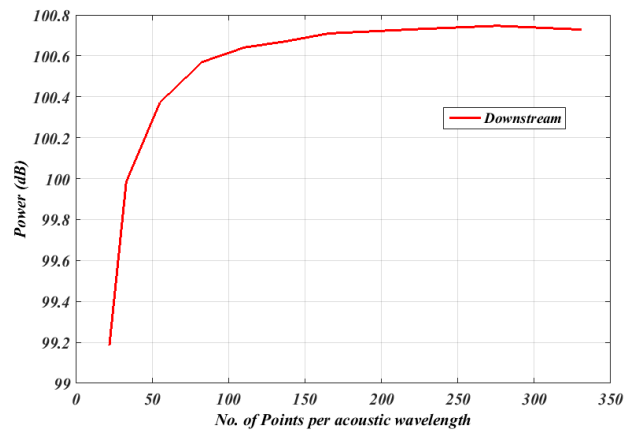

(b) Downstream power

FIGURE C.2: Grid sensitivity on a zero-staggered Flat plate for incoming transverse mode $\mathrm{m}=40$ at $\mathrm{M}=0.48$

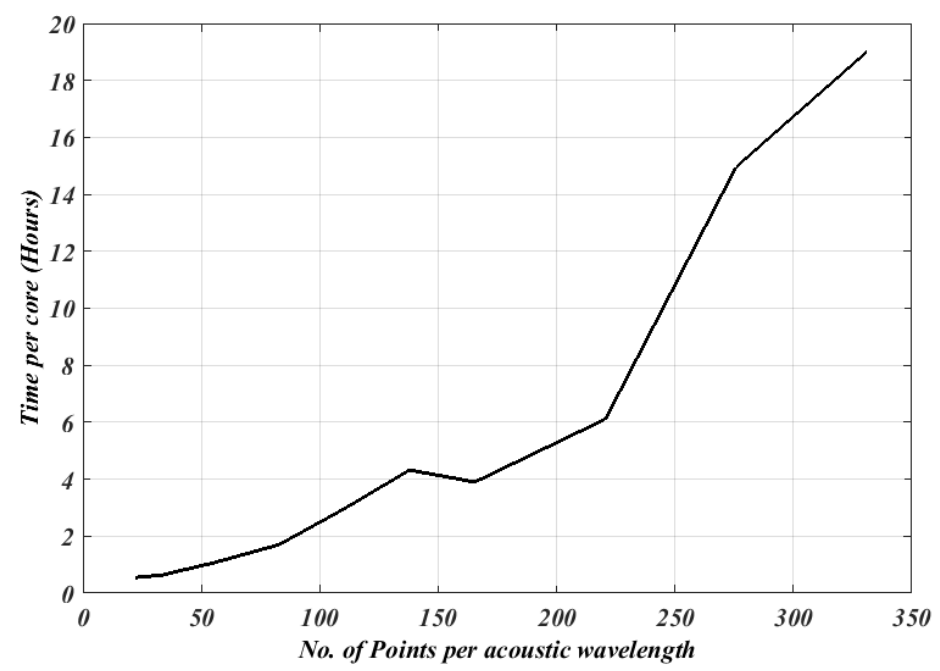

FIGURE C.3: CPU Hours against mesh density

per wavelength as shown in figure C.2a. Figure C.2b shows the variation of downstream acoustic power with mesh density. The variation of downstream power is very small and above 150 points per wavelength mesh density we find a reasonable convergence within a error of $0.1 \mathrm{~dB}$.

Figure C.3 plots the computational time against mesh density. It is evident from the figure C.2, the optimum mesh density is around 150 points per wavelength. The computational time above mesh density 150 increases drastically. Hence, all the results presented in Chapter 3 are performed on optimum mesh density of 150 points per wavelength.

\section{Grid alignment studies}

A similar grid definition is used in the second validation case (shown in section 3.5.2) of staggered flat plate cascade. The grid is skewed in the direction of wave propagation and the skewness angle $\alpha$ introduces a small amount of dissipation in the propagation. The dissipation depends on the alignment of the grid and the wave propagation. Figure C.4 shows the dissipation of incoming vortical velocity amplitude in the angle for three different skewness angles $\alpha$. The dissipation is observed to be relatively lower as the grid is align in 


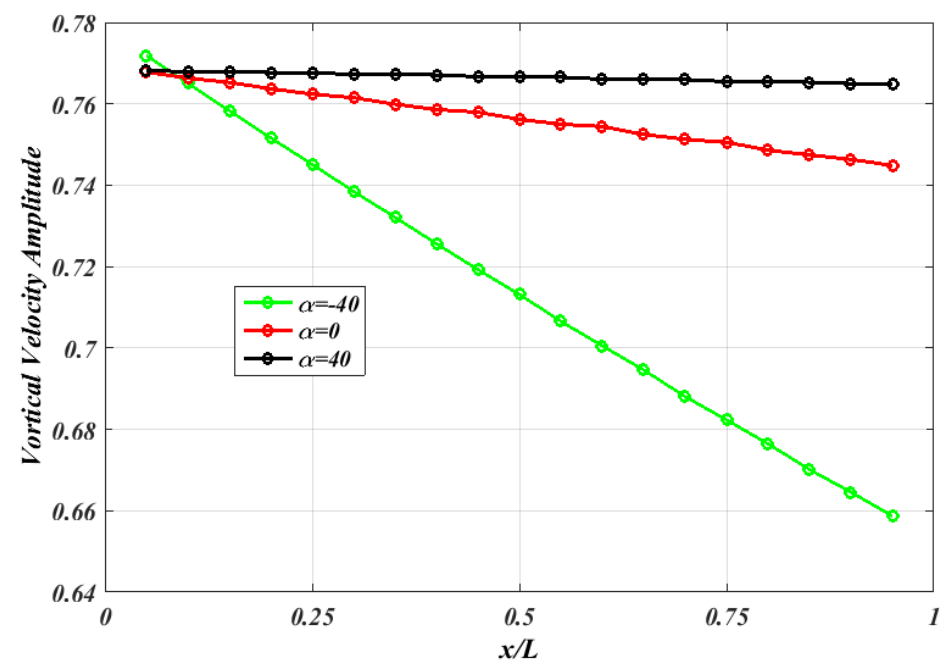

FIGURE C.4: Influence of grid alignment on dissipation

the direction of flow $(\alpha=40)$ and vice-versa. This dissipation is corrected for the upstream and downstream radiation power calculations due to linearity of the current approach. 



\section{Appendix D}

\section{Additional serration results}

\section{D.1 Modifying realistic OGV geometry for BB reduction}

The current section demonstrate the BB noise reduction on a realistic OGV geometry by modifying the leading edge nose geometry and by addition of leading edge serrations at the aerofoil LE. The baseline OGV geometry is a typical Rolls Royce modular fan rig ' 44 blade' geometry of $5 \%$ thickness relative to chord. The chord and span of the baseline aerofoil is $150 \mathrm{~mm}$ and $450 \mathrm{~mm}$. Figure D.1 shows the schematic of the various aerofoil profiles tested in the current study. A total of 4 aerofoil configurations are tested as shown in figure D.1. It has been shown in the Chapter 2, leading edge nose profile is the important parameter for thin aerofoils. The configuration tested in the current study as describes as below:

1. Baseline aerofoil

2. Thrice the leading edge nose radius relative to baseline aerofoil

3. Thrice the leading edge nose radius relative to baseline aerofoil and location of maximum thickness is moved to $30 \%$ from $50 \%$

4. Leading edge serrations with serration amplitude $h / c_{0}=0.033$ and serration wavelength $\lambda / c_{0}=0.4$.

Figure D.2 shows the sound power reductions relative to baseline aerofoil versus non-dimensional frequency $f c_{0} / U$ for all the above mentioned configurations at two jet velocities of $20 \mathrm{~m} / \mathrm{s}$ and $60 \mathrm{~m} / \mathrm{s}$. For a small amplitude serrations we still get better noise reductions at low frequency than compared to other two configurations. Changes in nose radius and nose profile has benefit at high frequencies than compared to serrated aerofoil. It is very evident from figure D.2, small modification in stream-wise direction (usage of serration) is more efficient than modification in transverse direction (nose profile) to reduce the broadband interaction noise. To show the noise reductions due to nose radius explicitly, 3 times nose radius relative to baseline configuration is consider in the present study. The profile with maximum thickness location at $30 \%$ has shown to have slightly $(<0.5 \mathrm{~dB})$ better noise reductions than
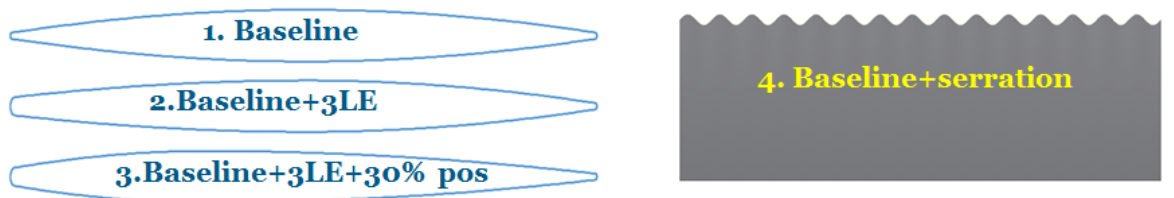

FIGURE D.1: A Schematic of the various leading edge OGV configurations tested (for serrated: $h / c_{0}=0.033$ and $\lambda / c_{0}=0.4$ ). 


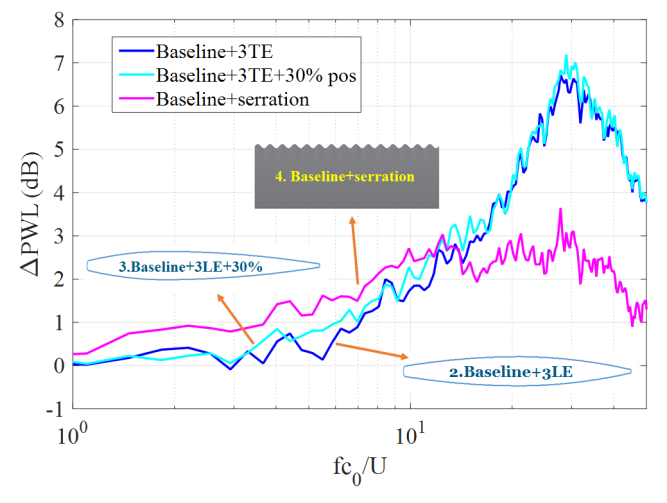

(a) $U=20 \mathrm{~m} / \mathrm{s}$.

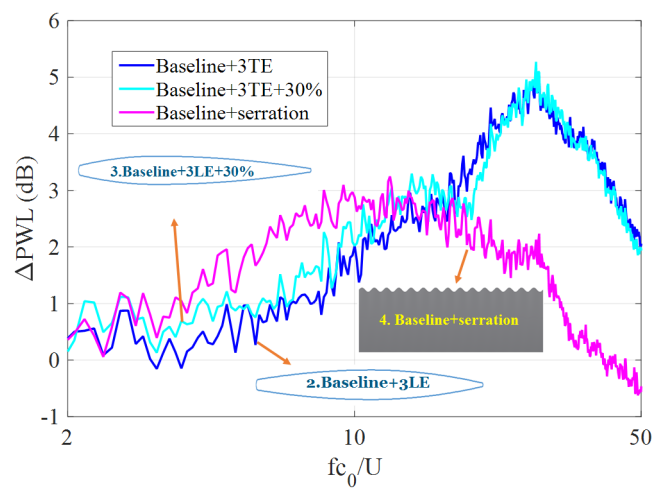

(b) $U=60 \mathrm{~m} / \mathrm{s}$.

FIGURE D.2: Acoustic performance of modified OGV geometry at two different jet velocity of 20 and $60 \mathrm{~m} / \mathrm{s}$.

compared to profile with 50\% maximum thickness location. This can be attributed to the extend of gust distortion due to nose profile very close to leading edge of the aerofoil. Thus, the present thesis focuses on leading edge serration geometries to achieve better low frequency attenuation.

\section{D.2 Leading edge serration geometries}

In this section, the acoustic performance of few additional geometries such as random and curved serrations are presented. The noise reductions are observed to deteriorate than compared to single-wavelength serrations but these results are presented here to understand the noise reduction phenomena.

\section{D.2.1 Random serrations}

Figure D.3 shows the geometry of the random profile applied to the airfoil leading edge, as defined in Equation D.1

$$
x_{n}=n \delta x, y_{n}=y_{0}+g\left(0, \sigma_{0}\right)
$$

where $x_{n}, y_{n}$ are the coordinates of the geometry, $g\left(0, \sigma_{0}\right)$ is the random variable that follows a normal distribution with zero mean and standard deviation $\sigma_{0}$ and $y_{0}$ is a constant chosen so that the surface area of the treated aerofoil is identical to that of the baseline aerofoil. Figure D.3 also has a close view of the leading edge serration whose $\delta x / c_{0}=0.02$. For the current random serration, standard deviation $\sigma_{0} / c_{0}$ is chosen as 0.067 . The sound power reductions are compared against single wavelength serration profile whose serration wavelength is equal to $\delta x / c_{0}$. In both the cases, the peak to peak distance $2 h / c_{0}$ is almost kept constant which is 0.33 .

Figure D.4 shows the sound power reduction versus non-dimensional frequency $f \sigma_{0} / U$ comparison between random leading edge serrations with sinusoidal single-wavelength serrations at a fixed peak-to-root $2 h / c_{0}$ distance of 0.33 . A good collapse of the noise reduction spectra is observed when plotted against non-dimensional frequency $f \sigma_{0} / U$. This 


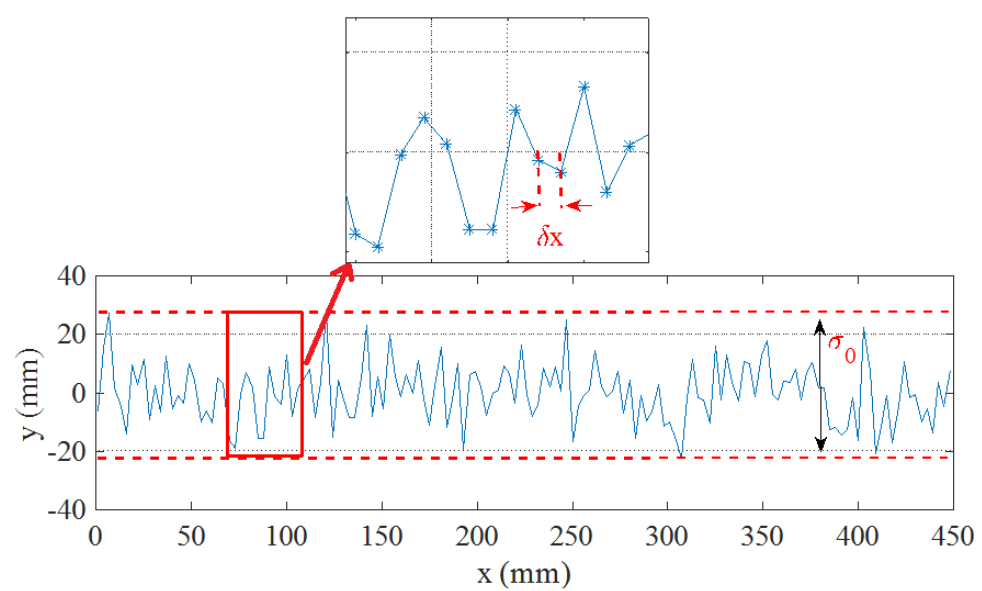

FIGURE D.3: A Schematic of the leading edge Slitted serrations.

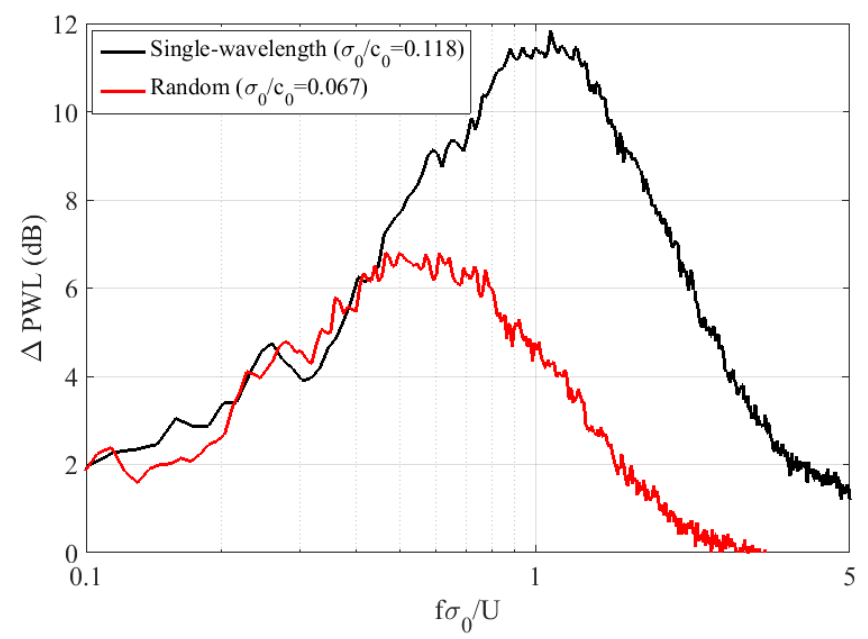

FIGURE D.4: Sound power reduction comparisons for a sinusoidal serration of amplitude $\left(h / c_{0}=0.167\right)$, wavelength $\lambda / c_{0}=0.02$ with random serrations $\left(\sigma_{0} / c_{0}=0.1\right)$ at jet velocity of $60 \mathrm{~m} / \mathrm{s}$

suggests the performance of the random serration depends on the average distance to the mean of the serration. Hence, the integrated phase variation along the random serrations is the key factor governing the noise reductions. As the random serrations have all variable serration amplitudes across the span of the aerofoil, the noise reductions are function of the standard deviation of the variations in the serration coordinates.

\section{D.2.2 Owl inspired serrations}

This section demonstrates the acoustic performance of owl inspired leading edge serrations designs. Curved and inclined leading edge profiles are investigated. A Schematic of a 'curved' and 'inclined' geometries are shown in the figure D.5.

Figure D.6 shows the sound power reduction versus non-dimensional frequency $f h / U$ comparison between curved and inclined leading geometries with sinusoidal serrations of serration amplitude $h / c_{0} 0.1$ and serration wavelength $\lambda / c_{0} 0.1$ at jet velocity $40 \mathrm{~m} / \mathrm{s}$. The acoustic 


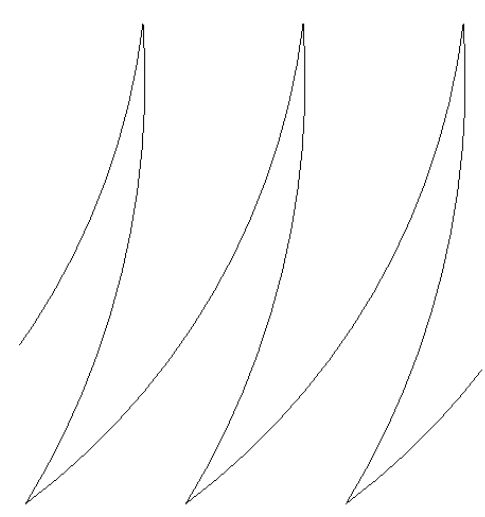

(a) Curved serrations.

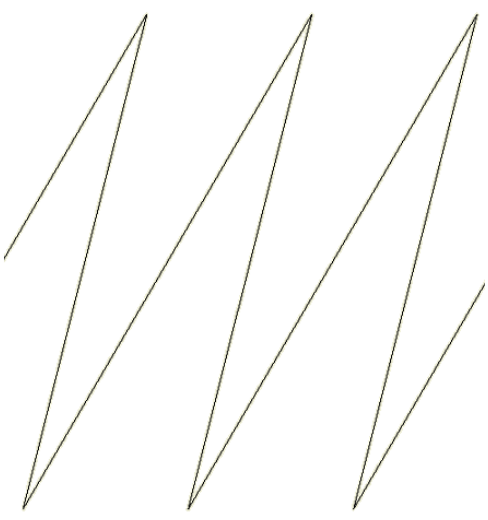

(b) Inclined serrations.

FIGURE D.5: A Schematic of the curved and inclined leading edge serrations.

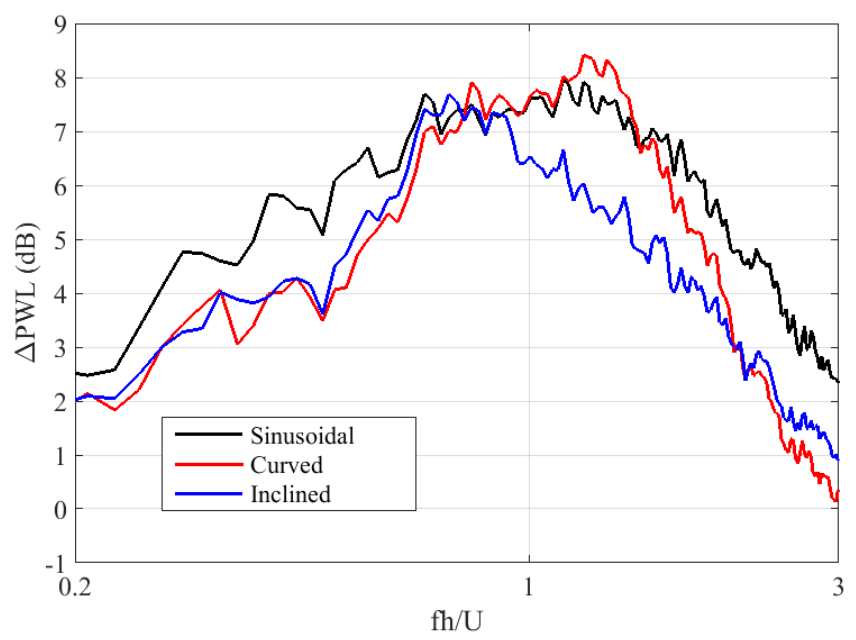

FIGURE D.6: Sound power reduction comparisons for a sinusoidal serration of amplitude $\left(h / c_{0}=0.1\right)$, wavelength $\lambda / c_{0}=0.1$ with curved and inclined serrations at jet velocity of $40 \mathrm{~m} / \mathrm{s}$

performance for both curved and inclined edge are almost identical. This can be due to almost similar phase change across the leading edge of the serration geometry. The noise reductions deteriorates for curved and inclined serrations than compared to sinusoidal serrations especially at lower non-dimensional frequency. This might be due to hidden inclined edge which is not seeing the flow directly. In case of sinusoidal serrations both the inclined edges are explored to mean flow which enhances the destructive interference across the inclined edge. Whereas in case of inclined or curved serrations, only one edge is exposed to mean flow directly resulting in reduced destructive interference.

\section{D.3 Combined leading and trailing edge serrations}

It was shown in the Chapter 4 the effectiveness of leading edge serrations on turbulenceaerofoil interaction noise is constrained by self-noise of the aerofoil. Many researchers, such 


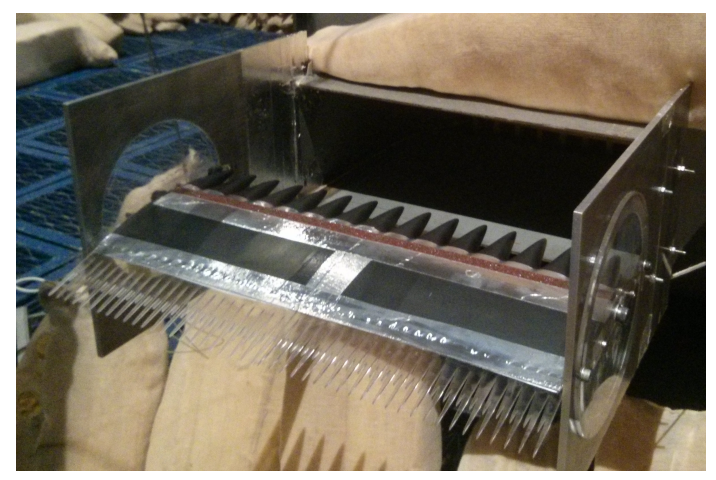

FIGURE D.7: A photograph of double serrated aerofoil mounted at the exit of nozzle.

as Gruber Gruber (2012); Gruber et al. (2013) has shown previously the usage of trailingedge serrations reduces trailing-edge noise. We also observed that the leading edge serrations plays a crucial role in reducing leading edge noise as well as substantial reduction in self-noise. In this current section, trailing edge serrations are used to reduce the self-noise further along with leading edge serrations.

\section{D.3.1 Aerofoil configuration}

NACA-65(12)10 is considered as baseline aerofoil for the present analysis. Leading edge serration profile considered in the current study is described by Eq 4.1. The serration wavelength of $\lambda / c_{0}=0.2$ and serration amplitude $h / c_{0}$ of 0.167 , is considered in the present study. In addition with the leading edge serrations, trailing edge serrations with dual-wavelength serrations, of wavelengths $\lambda / c_{0} 0.033 \& 0.067$ and the amplitude $h / c_{0} 0.167$, are attached to the baseline airfoil. For the economy and ease of manufacture, the trailing edge serrations are fabricated on a flat plate. A photograph of double serrated aerofoil mounted on the nozzle exit is shown in the Figure D.7.

\section{D.3.2 Power spectra comparison}

The preliminary results for combined serrations on NACA-65 aerofoil at a jet velocity $U 60$ $\mathrm{m} / \mathrm{s}$ are presented in this section. Figures D.8 shows the comparison of total sound power reductions versus non-dimensional frequency $f c_{0} / U$ between leading edge serration alone with the combined leading and trailing edge serrations. The same figure clearly shows the trailing edge serrations are effective from the frequency where the self-noise starts dominating. This clearly shows that the combined effects of trailing and leading edge serrations is commutative. More experiments need to be performed to understand the sensitivities on combined serrations. The reason for the decrease in noise reductions at low frequencies $\left(f c_{0} / U<2.5\right)$ by the addition of trailing edge serrations is currently unknown. A more detailed study to measure the leading edge and trailing edge noise reductions separately has to performed in future to understand the combined phenomena. 


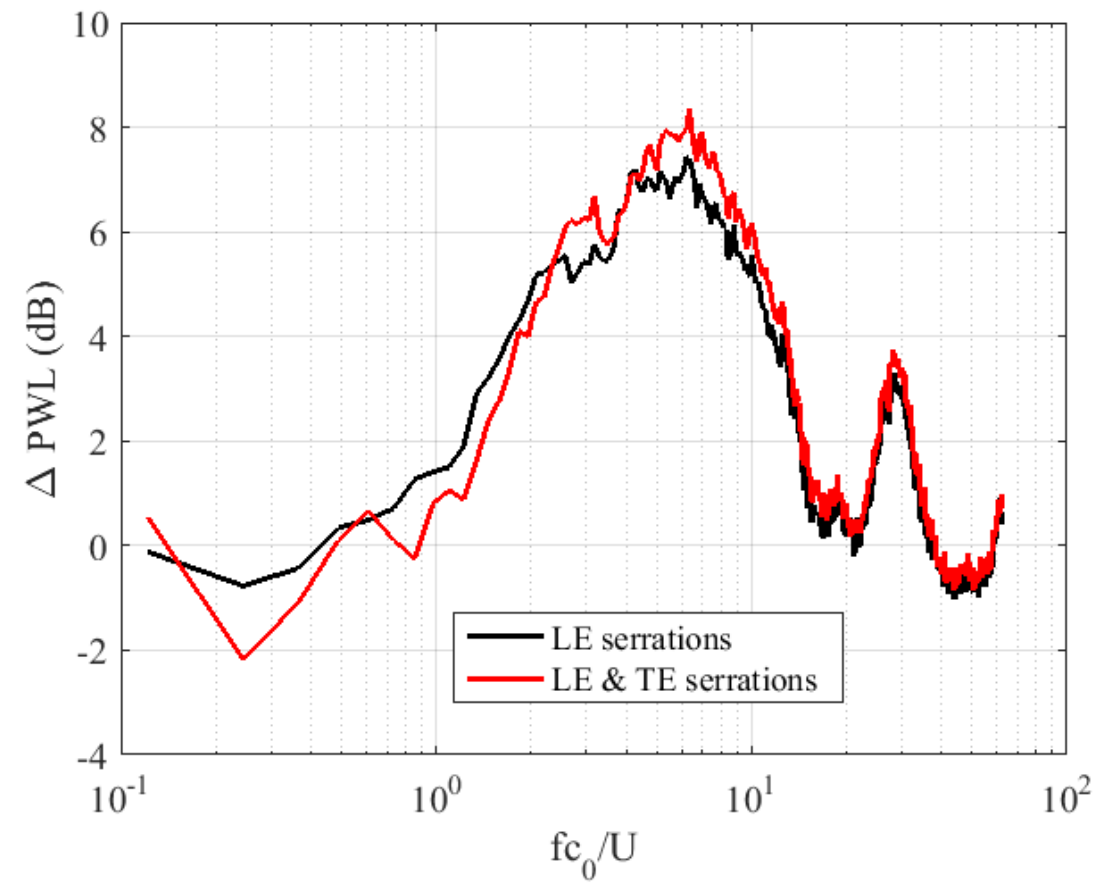

FIGURE D.8: Sound power reduction level $(\triangle P W L)$ for leading edge serration amplitude $h / c_{0}=0.167$ and serrated wavelength $\left(\lambda / c_{0}\right)$ of 0.2 along with double-wavelength trailing edge serration $\lambda_{1} / c_{0}=0.033 \& \lambda_{1} / c_{0}=0.066$ at jet velocity $60 \mathrm{~m} / \mathrm{s}$ and $A o A=0^{\circ}$ 


\section{Bibliography}

Adamczyk, J. J. (1974). The passage of an infinite swept airfoil through an oblique gust. NASA Contractor Report 2395.

Amiet, R. (1975). Acoustic radiation from an airfoil in a turbulent stream. Journal of Sound and Vibration, 41(4):407-420.

Amiet, R. K. (1974). Compressibility effects in unsteady thin-airfoil theory. AIA Journal, 12(2):252-255.

Amiet, R. K. (1976). Noise due to turbulent flow past a trailing edge. Journal of Sound and Vibration, 47(3):387-393.

Astley, R., Agarwal, A., Holland, K., Joseph, P., Self, R., Smith, M., Sugimoto, R., and Tester, B. (2007). Predicting and reducing aircraft noise. In 14th International Congress on Sound and Vibration.

Atassi, H. M. (1984). The sears problem for a lifting airfoil revisited - new results. Journal of Fluid Mechanics, 141:109-122.

Atassi, H. M., Subramaniam, S., and Scott, J. R. (1990). Acoustic radiation from lifting airfoils in compressible subsonic flow. In 13th Aeroacoustics Conference.

Ayton, L. J. (2016). An analytic solution for gust-aerofoil interaction noise including effects of geometry. IMA Journal of Applied Mathematics, 1-25:00.

Bachmann, T., Klän, S., Baumgartner, W., Klaas, M., Schröder, W., and Wagner, H. (2007). Morphometric characterisation of wing feathers of the barn owl tyto alba pratincola and the pigeon columba livia. Frontiers in Zoology, 4(1):23.

Billson, M. (2002). Semi-analytical methodologies for airfoil noise prediction. $\mathrm{PhD}$ thesis, Chalmers University of Technology.

Blake, W. K. (1970). Turbulent boundary-layer wall-pressure fluctuations on smooth and rough walls. Journal of Fluid Mechanics, 44(04):637-660.

Blandeau, V. (2011). Aerodynamic broadband noise form contra-rotating open rotors. PhD thesis, University of Southampton.

Brooks, T. F., Pope, D. S., and Marcolini, M. A. (1989). Airfoil self-noise and prediction, volume 1218. National Aeronautics and Space Administration, Office of Management, Scientific and Technical Information Division.

Campobasso, M. S. and Giles, M. B. (2003). Effects of flow instabilities on the linear analysis of turbomachinery aeroelasticity. Journal of Propulsion and Power, 19-4:693-703.

Chaitanya, P., Gill, J., Narayanan, S., Joseph, P., Vanderwel, C., Zhang, X., and Ganapathisubramani, B. (2015a). Aerofoil geometry effects on turbulence interaction noise. In 21st AIAA/CEAS Aeroacoustics Conference, number AIAA-2015-2830. 
Chaitanya, P., Joseph, P., Narayanan, S., Vanderwel, C., Turner, J., Kim, J. W., and Ganapathisubramani, B. (2017). Performance and mechanism of sinusoidal leading edge serrations for the reduction of turbulence-aerofoil interaction noise. Journal of Fluid Mechanics (accepted), doi:10.1017/jfm.2016.95.

Chaitanya, P., Narayanan, S., Joseph, P., , and Kim, J. W. (2016). Leading edge serration geometries for significantly enhanced leading edge noise reductions. In 22nd AIAA/CEAS Aeroacoustics Conference, AIAA 2016-2736, number AIAA 2016-2736.

Chaitanya, P., Narayanan, S., Joseph, P., Vanderwel, C., Turner, J., Kim, J. W., and Ganapathisubramani, B. (2015b). Broadband noise reduction through leading edge serrations on realistic aerofoils. In 21st AIAA/CEAS Aeroacoustics Conference, number AIAA 2015-2202.

Chaitanya, P., Stalnov, O., Joseph, P., and Leung, R. (2015). On the robustness of the tno model for aerofoil self-noise prediction. In 21st AIAA/CEAS Aeroacoustics Conference, number AIAA 2015-2367.

Cheong, C., Joseph, P., and Lee, S. (2006). High frequency formulation for the acoustic power spectrum due to cascade-turbulence interaction. Journal of the Acoustical Society of America, 119(1):108-122.

Chong, T. P., Joseph, P., and Davies, P. O. A. L. (2008). A parametric study of passive flow control for a short, high area ratio $90^{\circ}$ curved diffuser. Journal of Fluids Engineering, 130(11):111104-12.

Chong, T. P., Vathylakis, A., McEwen, A., Kemsley, F., Muhammad, C., and Siddiqi, S. (2015). Aeroacoustic and aerodynamics performance of an aerofoil subjected to sinusoidal leading edges. In 21st AIAA/CEAS Aeroacoustics Conference, number AIAA 2015-2200.

Clair, V., Polacsek, C., Garrec, T. L., Reboul, G., Gruber., M., and Joseph, P. (2013). Experimental and numerical investigation of turbulence-airfoil noise reduction using wavy edges. AIAA Journal, 51(11):2695-2713.

Collins, F. G. (1981). Boundary layer control on wings using sound and leading edge serrations. American Institute of aeronautics and Astronautics Journal, 19(2):129-130.

Devenport, W. J., Staubs, J. K., and Glegg, S. A. L. (2010). Sound radiation from real airfoils in turbulence. Journal of Sound and Vibration, 329:3470-3483.

Dieste, M. (2011). Random-Vortex-Particle Methods Applied to Broadband Fan Interaction Noise. $\mathrm{PhD}$ thesis, University of Southampton.

Evers, I. and Peake, N. (2002). On sound generation by the interaction between turbulence and a cascade of airfoils with non-uniform mean flow. Journal of Fluid Mechanics, 463:2552 .

Ewert, R. (2008). Broadband slat noise prediction based on caa and stochastic sound sources from a fast random particle-mesh (rpm) method. Computers Fluids, 37:369-387.

Favier, J., Pinelli, A., and Piomelli, U. (2012). Control of the separated flow around an airfoil using awavy leading edge inspired by humpback whale flippers. Comptes Rendus Mécanique, 340(1-2):107-114.

Fish, F. E. and Lauder, G. V. (2006). Passive and active flow control by swimming fishes and mammals. Annual Review of Fluid Mechanics, 38:193-224. 
Fish, F. E., Weber, P. W., and Murray, M. M. andHowle, L. E. (2011). The tubercles on humpback whales' flippers: Application of bio-inspired technology. Integrative and Comparative Biology, 51(1):203-213.

Gea-Aguilera, F., Gill, J. R., Angland, D., and Zhang, X. (2017). Wavy leading edge airfoils interacting with anisotropic turbulence read more: https://arc.aiaa.org/doi/abs/10.2514/6.2017-3370. In 23rd AIAA/CEAS Aeroacoustics Conference, number AIAA 2017-3370.

Gea-Aguilera, F., Zhang, X., Chen, X., Gill, J., and Langlois§, T. N. (2015). Synthetic turbulence methods for leading edge noise predictions. In 21st AIAA/CEAS Aeroacoustics Conference, number AIAA-2015-2670.

Gershfeld, J. (2004). Leading edge noise from thick foils in turbulent flows. Journal of acoustical society of America, 116:1416-1426.

Giacche, D., Xu, L., Coupland, J., and Wilson, A. G. (2011). A comparison between postprocessing methods applied to rotor-stator interaction tone noise problems. AIAA Journal, 49(6):1214-1229.

Gill, J. (2015). Broadband Noise Generation of a Contra-Rotating Open Rotor Blade. PhD thesis, University of Southampton.

Gill, J., Zhang, X., and Joseph, P. (2013). Symmetric airofils geometry effects on leading edge noise. Journal of acoustical society of America, 34(4):2669-2680.

Glegg, S. (1998). Broadband fan noise generated by small turbulence lenght scale. Technical report.

Glegg, S. (1999). The response of a swept blade row to a three-dimensional gust. Journal of Sound and Vibration, 227(1):29-64.

Glegg, S. and Devenport, W. (2009). Unsteady loading on an airfoil of arbitrary thickness. Journal of Sound and Vibration, 319:1252-1270.

Glegg, S. and Devenport, W. (2010). Panel methods for airfoils in turbulent flow. Journal of Sound and Vibration, 329:3709-3720.

Goldstein, M. and Atassi, H. (1976). A complete second-order theory for the unsteady flow about an airfoil due to a periodic gust. Journal of Fluid Mechanics, 74:741-765.

Grace, S. (2001). Unsteady blade response : the BVI model vs. the gust model. In 7th AIAA/CEAS Aeroacoustics Conference, number AIAA-2001-2209.

Graham, J. (1970). Similarity rules for thin airfoils in non-stationary subsonic flows. Journal of Fluid Mechanics, 3 (4):753-766.

Gruber, M. (2012). Airfoil noise reduction by edge treatments. PhD thesis, University of Southampton, ISVR.

Gruber, M., Joseph, P., and Azarpeyvand, M. (2013). An experimental investigation of novel trailing edge geometries on airfoil trailing edge noise reduction. In 19th AIAA/CEAS Aeroacoustics Conference, number AIAA-2013-2011.

Haeri, S., Kim, J. W., Narayanan, S., and Joseph, P. (2014). 3d calculations of aerofoilturbulence interaction noise and the effect of wavy leading edges. In 20th AIAA/CEAS Aeroacoustics Conference, number 2014-2325, Atlanta, GA,. 
Hainaut, T., Gabard, G., and Clair, V. (2016). A caa study of turbulence distortion in broadband fan interaction noise. In 22nd AIAA/CEAS Aeroacoustics Conference, number AIAA 2016-2839.

Hall, A. M., Atassi, O. V., and Gilson, J. (2011). Effects of leading-edge thickness on highspeed airfoil-turbulence interaction noise. In 17th AIAA/CEAS Aeroacoustics Conference, Portland, Oregon, number AAIA Pap. 2011-2861.

Hansen, K., Kelso, R., and Dally, B. (2011). Performance variations of leading-edge tubercles for distinct airfoil profiles. AIAA Journal, 49(1):185-194.

Hanson, D. and Horan, K. (1998). Turbulence/cascade interaction: spectra of inflow, cascade response and noise. In Proceedings of the 4th AIAA/CEAS Aeroacoustics Conference, number AIAA-98-2319.

Hersh, A., Soderman, P., and Hayden, R. (1974). Investigation of acoustic effets of leading edge serrations on airfoils. Journal of Aircraft, 11(4):197-202.

Howe, M. (2001). Unsteady lift and sound produced by an airfoil in a turbulent boundary layer. J Fluids and Structures, 15:207-225.

Jenkins, G. (2013). Models for the prediction of rear-arc and forward-arc fan broadband noise in turbofan engines. PhD thesis, University of Southampton.

Johari, H., Henoch, C., Custodio, D., and Levshin, A. (2007). Effects of leading-edge protuberances on airfoil performance. AIAA Journal, 45(11):2634-2642.

Kaji, S. and Okazaki, T. (1972). Cascade flutter in compressible flow. Transaction of the Japan Society of Mechanical Engineers, 38:1023-1033.

Kim, J., Haeri, S., and Joseph, P. (2016). On the reduction of aerofoil-turbulence interaction noise associated with wavy leading edges. Journal of Fluid Mechanics, 792:526-552.

Kucukcoskun, K., Christophe, J., Schram, C., and Tournour, M. (2013). Broadband scattering of the turbulence-interaction noise of a stationary airfoil: experimental validation of a semi-analytical model. International Journal of Aeroacoustics, 12(1+2):83-102.

Lane, F. and Friedman, M. (1958). Unsteady aerodynamics of vortical flows: early and recent developments. Technical report, .NACA-TN-4136.

Lau, A. S., Haeri, S., and Kim, J. W. (2013). The effect of wavy leading edges on aerofoil-gust interaction noise. Journal of Sound and Vibration, 332:6234-6253.

Leung, R., Chaitanya, P., and Joseph, P. (2016). Effect of aerofoil thickness on trailing edge noise. In 22nd AIAA/CEAS Aeroacoustics Conference, number AIAA 2016-2815.

Liu, R., Ting, D. S.-K., and Rankin, G. W. (2004). On the generation of turbulence with a perforated plate. Experimental Thermal and Fluid Science, 28:307-316.

Liu, X., Jawahar, H. K., Azarpeyvand, M., and Theunissen, R. (2015). Aerodynamic and aeroacoustic performance of serrated airfoils. In 21st AIAA/CEAS Aeroacoustics Conference, number AIAA 2015-2201.

Lockard, D. and Morris, P. (1998). Radiated noise from airfoils in realistic mean flows. AIAA Journal, 36(6):907-914.

Lysak, P. D. (2011). Unsteady lift of thick airfoils in incompressible turbulent flow. PhD thesis, The Pennsylvania state University. 
Lysak, P. D., Capone, D. E., and Jonson, M. L. (2013). Prediction of high frequency gust response with airofoil thickness effects. Journal of Fluids and Structures, 39:258-274.

Lyu, B., Azarpeyvand, M., and Sinayoko, S. (2016). Noise prediction for serrated leadingedges. In 22nd AIAA/CEAS Aeroacoustics Conference, AIAA 2016-2740, number AIAA 20162740.

Mani, R. and Horvay, G. (1970). Sound transmission through blade rows. Journal of Sound and Vibration, 12(1):59-83.

Miklosovic, D. S., Murray, M. M., and Howle, L. (2007). Experimental evaluation of sinusoidal leading edges. Journal of Aircraft, 44(4):1404-1408.

Miklosovic, D. S., Murray, M. M., Howle, L. E., and Fish, F. E. (2004). Leading-edge tubercles delay stall on humpback whale (megaptera novaeangliae) flippers. Physics of Fluids, 16(5).

Moinier, P. (1999). Algorithm Developments for an Unstructured Viscous Flow Solver. PhD thesis, Oxford University.

Moinier, P. and Giles, M. B. (2005). Eigenmode analysis for turbomachinery applications," journal of propulsion and power. Journal of the American Helicopter Society, 21(6):973-978.

Moinier, P., Giles, M. B., and Coupland, J. (2007). Three-dimensional non-reflecting boundary conditions for swirling flows in turbomachinery. Journal of Propulsion and Power, 23(5):981-986.

Moreau, S., Roger, M., and Jurdic, V. (2005). Effect of angle of attack and airfoil shape on turbulence ingestion noise. In 11th AIAA/CEAS Aeroacoustics Conference, number AIAA2005-2973.

Moriarty, P. J., Guidati, G., and Migliore, P. (2005). Prediction of turbulent inflow and trailing-edge noise for wind turbines. In 11th AIAA/CEAS Aeroacoustics Conference.

Myers, M. and Kerschen, E. (1995). Influence of incident angle on sound generation by airfoils interacting with high-frequency gusts. Journal of Fluid Mechanics, 292:271-304.

Myers, M. and Kerschen, E. (1997). Influence of camber on sound generation by airfoils interacting with high-frequency gusts. Journal of Fluid Mechanics, 353:221-259.

Narayanan, S., Chaitanya, P., Haeri, S., Joseph, P., Kim, J. W., and Polacsek, C. (2015). Airfoil noise reductions through leading edge serrations. Physics of Fluids, 27(025109).

Narayanan, S., Joseph, P., Haeri, S., Kim, J. W., Chaitanya, P., and Polacsek, C. (2014). Noise reduction studies from the leading edge of serrated flat plates. In 20th AIAA/CEAS Aeroacoustics Conference, number 2014-2320.

Oerlemans, S. and Migliore, P. (2004). Aeroacoustic wind tunnel tests of wind turbine airfoils. In 10th AIAA/CEAS Aeroacoustics Conference, Manchester, UK.

Olsen, W. and Wagner, J. (1982). Effect of thickness on airfoil surface noise. In AIAA 82-4068.

Paterson, R. and Amiet, R. (1977). Noise and surface pressure response of an airfoil to incident turbulence. Journal of aircraft, 14(8):729-736.

Peake, N. and Kerschen, E. (1997). Influence of mean loading on noise generated by the interaction of gust with plate cascade: upstream radiation. Journal of Fluid Mechanics, 347:315-346. 
Polacsek, C., Clair, V., Garrec, T. L., Reboul, G., and Jacob, M. C. (2015). Numerical predictions of turbulence/cascade-interaction noise using computational aeroacoustics with a stochastic model. AIAA Journal, 53:3551-3566.

Roach, P. E. (1987). The generation of nearly isotropic turbulence by means of grids. Heat and Fluid Flow, 8(2):82-92.

Roger, M. (2010). Analytical modeling of the potential-interaction noise of a rotor due to a downstream rod. In 13th International Symposium on Transport Phenomena and Dynamics of Rotating Machinery.

Roger, M., Schram, C., and Santana, C. . L. D. (2013). Reduction of airfoil turbulenceimpingement noise by means of leading-edge serrations and/or porous materials. In AIAA/CEAS Aeroacoustics Conference, number 2013-2108, Berlin, Germany.

Rostamzadeh, N., Kelso, R. M., Dally, B. B., and Hansen, K. L. (2013). The effect of undulating leading-edge modifications on naca 0021 airfoil characteristics. PHYSICS OF FLUIDS, 25:117101-19.

Santana, L., Christophe, S., and Wim, D. (2016a). Low-frequency extension of amiets theory for compact airfoil noise predictions. Journal of Sound and Vibration, 372:342-356.

Santana, L., Julien, C., Christophe, S., and Wim, D. (2016b). A rapid distortion theory modified turbulence spectra for semi-analytical airfoil noise prediction. Journal of Sound and Vibration, 383:349-363.

Santana, L., Schram, C., and Desmet, W. (2012). Panel method for turbulence-airofoil interaction noise prediction. In 18th AIAA/CEAS Aeroacoustic Conference, number AIAA 20122073.

Schwarzschild, K. (1902). Die beugung und polarisation des lichts durch einen spalt. Mathematische Annalen, 55:177-247.

Sears, W. (1941). Some aspects of non-stationary airfoil theory and its practical applications. Journal of Aeronautical Sciences, 8(3):104-108.

Shahpar, S. and Lapwroth, L. (2003). Padram: Parametric design an rapid meshing system for turbomachinery optimisation. In ASME Turbo Expo, number GT-2003-38698.

Skillen, A., Revell, A., Pinelli, A., Piomelli, U., and Favier, J. (2014). Flow over a wing with leading-edge undulations. AIAA Journal, pages 1-9.

Smith, S. (1972). Discrete frequency sound generation in axial flow turbomachines. Aeronautical Research Council Reports and Memoranda 3709.

Soderman, P. (1972). Aerodynamic effects of leading-edge serrations on a two- dimensional airfoil. Technical report, NASA TM X-2643.

Stalnov, O., Chaitanya, P., and Joseph, P. (2015). Prediction of broadband trailing-edge noise based on blake model and amiet theory. In 21st AIAA/CEAS Aeroacoustics Conference, number AIAA 2015-2526.

Stanway, M. J. (2008). Hydrodynamic Effects of Leading-Edge Tubercles on Control Surfaces and in Flapping Foil Propulsion. PhD thesis, Massachusetts Inst. of Technology, Cambridge, MA.

Tsai, C.-T. (1992). Effect of airfoil thickness on high-frequency gust interaction noise. PhD thesis, University of Arizona. 
Turner, J., Kim, J. W., Chaitanya, P., and Joseph, P. (2016). Towards understanding aerofoils with dual-frequency wavy leading edges interacting with vortical disturbances. In 22nd AIAA/CEAS Aeroacoustics Conference, AIAA 2016-2951, number AIAA 2016-2951.

Turner, J. M. and Kim, J. W. (2017). Aeroacoustic source mechanisms of a wavy leading edge undergoing vortical disturbances. Journal of Fluid Mechanics, 811:582-611.

Van Nierop, E., Alben, S., and Brenner, M. (2008). How bumps on whale flippers delay stall: An aerodynamic model. Physical Review Letters, 100(5):1-4.

Whitehead, D. (1965). Bending flutter of unstalled cascade blades at finite deflection. Technical report, Reports and Memoranda R. \& M. No. 3386, Ministry of Aviation, Aeronautical Reseach Council, London,.

Whitehead, D. (1987). AGARD Manual on Aeroelasticity in Axial-Flow Turbomachines Volume 1 Unsteady Turbomachinery Aerodynamics, chapter Classical two-dimensional methods, pages 3.1-3.30.

Yoon, H. S., Hung, P. A., Jung, J. H., and Kim, M. C. (2011). Effect of the wavy leading edge on hydrodynamic characteristics for flow around low aspect ratio wing. Computer $\mathcal{E}$ fluids, 49:276-289.

Zhang, M. M., Wang, G. F., and Xu, J. Z. (2013). Aerodynamic control of low-reynoldsnumber airfoil with leading-edge protuberances. AIAA Journal, 51(8):1960-1971. 